\title{
Functionalisation of Alkenes Through Telescoped Continuous Flow Aziridination Processes
}

\author{
Nathanael Hsueh, Guy J. Clarkson and Michael Shipman* \\ Department of Chemistry, University of Warwick, Gibbet Hill Road, Coventry, CV4 7AL, UK. Fax: +44 \\ 2476 524112; Tel: +44 2476 523186; E-mail: m.shipman@warwick.ac.uk
}

\section{Supporting Information}

1. General details

2. Synthesis of o-propoxyaryliminoiodanes

3. Optimization of conditions for aziridination

4. Flow synthesis of aziridines $\mathbf{2 a - I}$

5. Alternative flow synthesis of $\mathbf{2} \mathbf{d}$ and $\mathbf{2} \mathbf{f}$ in a tube reactor

$S 14-S 15$

6. Flow synthesis of amines 3a-r by telescoped aziridination/ring-opening

$S 15-S 25$

7. Flow synthesis of imidazolines $\mathbf{4 a - c}$ by telescoped aziridination/ring-expansion

$S 25-S 27$

8. X-ray crystallography and depiction of $\mathbf{3 h}$

9. ${ }^{1} \mathrm{H}$ and ${ }^{13} \mathrm{C}$ NMR spectra of SM1, SM2, 1a-d, 2a-I, 3a-r, and 4a-c 


\section{General details}

Several custom flow reaction set-ups were used as detailed (see S8, S14 and S15). Batch reactions were performed under an atmosphere of dry nitrogen in oven dried glassware. Anhydrous solvents were purchased in Sure/Seal ${ }^{\mathrm{TM}}$ bottles from Sigma-Aldrich. All other solvents and reagents were used as received or purified by standard protocols. Column chromatography was carried out using $40-64 \mu \mathrm{m}$ particle size silica gel.

Nuclear magnetic resonance (NMR) spectra were recorded on a Bruker Spectrospin DPX300 or HD300 $\left({ }^{1} \mathrm{H}\right.$ at $300 \mathrm{MHz}$ and ${ }^{13} \mathrm{C}$ at $\left.75 \mathrm{MHz}\right)$; Bruker Spectrospin DPX400 or HD400 $\left({ }^{1} \mathrm{H}\right.$ at $400 \mathrm{MHz}$ and ${ }^{13} \mathrm{C}$ at $\left.100 \mathrm{MHz}\right)$; or a Bruker Spectrospin HD500 $\left({ }^{1} \mathrm{H}\right.$ at 500 $\mathrm{MHz}$ and ${ }^{13} \mathrm{C}$ at $125 \mathrm{MHz}$ ). Chemical shifts are reported in ppm using TMS as internal standard. Peak multiplicities were recorded as singlet (s), doublet (d), triplet (t), quartet (q), quintet (quint), multiplet $(m)$, and coupling constants $(J)$ are reported in Hertz. NMR assignments were deduced using 2D experiments (COSY, HMBC and HMQC). Infrared spectra were recorded on a Bruker Alpha Platinum ATR spectrometer. Low resolution mass spectra were recorded on an Bruker Esquire 2000 platform with electrospray ionization. High resolution mass spectra were obtained on a Bruker MicroTOF spectrometer 


\section{Synthesis of o-propoxyaryliminoiodanes}

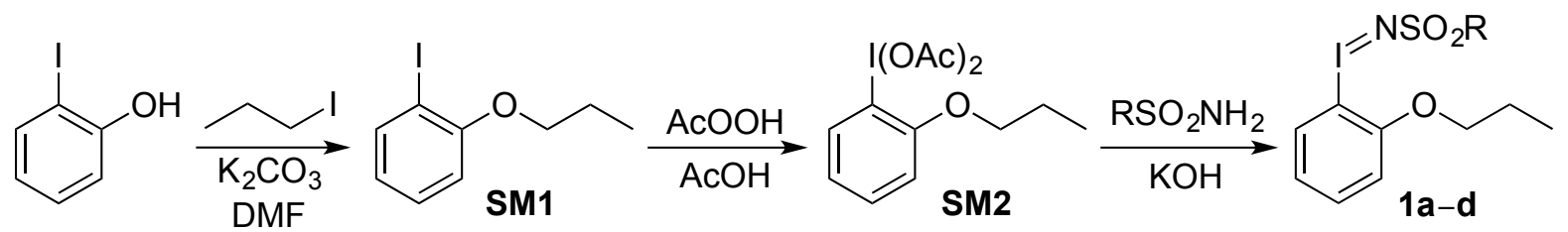<smiles>CCCOc1ccccc1I</smiles>

1-lodo-2-propoxybenzene SM1. 2-lodophenol (2.31 g, $10.5 \mathrm{mmol})$ was dissolved in DMF $(20 \mathrm{ml}) . \mathrm{K}_{2} \mathrm{CO}_{3}(7.78 \mathrm{~g}, 56.3 \mathrm{mmol})$ was added, and the mixture stirred for $10 \mathrm{~min}$. 1-lodopropane $(2.57 \mathrm{~g}, 15.1 \mathrm{mmol})$ was added, and the mixture stirred at $50{ }^{\circ} \mathrm{C}$ for $5 \mathrm{~h}$. At which point a yellow solution containing solids was obtained. The solvent was removed under high vacuum on a rotary evaporator, saturated aq $\mathrm{NaCl}(50 \mathrm{ml})$ was added, and the mixture extracted with EtOAc $(5 \times 10 \mathrm{ml})$. The organic extracts were combined, dried with $\mathrm{MgSO}_{4}$, filtered and the solvent removed in vacuo. Column chromatography $\left(5: 1^{n}\right.$ hexane:EtOAc) gave SM1 $(2.20 \mathrm{~g}, 8.39 \mathrm{mmol}, 80 \%)$ as a clear oil. IR (film): $2963(\mathrm{C}-\mathrm{H}), 2935(\mathrm{C}-\mathrm{H}), 2875(\mathrm{C}-\mathrm{H}), 1580(\mathrm{C}=\mathrm{C}) \mathrm{cm}^{-1} . \delta_{\mathrm{H}}(500$ $\left.\mathrm{MHz}, \mathrm{CDCl}_{3}\right): 7.79-7.74(1 \mathrm{H}, \mathrm{m}, \mathrm{ArH}), 7.31-7.25(1 \mathrm{H}, \mathrm{m}, \mathrm{ArH}), 6.82-6.78(1 \mathrm{H}, \mathrm{m}, \mathrm{ArH})$, $6.72-6.67(1 \mathrm{H}, \mathrm{m}, \mathrm{Ar} H), 3.98\left(2 \mathrm{H}, \mathrm{t}, J=7.0 \mathrm{~Hz}, \mathrm{OCH}_{2} \mathrm{CH}_{2} \mathrm{CH}_{3}\right), 1.87(2 \mathrm{H}$, sextuplet, $J=7.0$ $\left.\mathrm{Hz}, \mathrm{OCH}_{2} \mathrm{CH}_{2} \mathrm{CH}_{3}\right), 1.10\left(3 \mathrm{H}, \mathrm{t}, J=7.0 \mathrm{~Hz}, \mathrm{OCH}_{2} \mathrm{CH}_{2} \mathrm{CH}_{3}\right) ; \delta_{\mathrm{C}}\left(125 \mathrm{MHz}, \mathrm{CDCl}_{3}\right): 157.6$ (C), $139.4(\mathrm{CH}), 129.4(\mathrm{CH}), 122.3(\mathrm{CH}), 112.1(\mathrm{CH}), 86.7(\mathrm{C}), 70.7\left(\mathrm{CH}_{2}\right), 22.6\left(\mathrm{CH}_{2}\right), 10.8$ $\left(\mathrm{CH}_{3}\right)$. Data consistent with published values. ${ }^{1}$

(2-Propoxyphenyl)- $\lambda^{3}$-iodanediyl diacetate SM2. To SM1 (2.20 g, 8.39 $20.1 \mathrm{mmol}$ ) dropwise over $1 \mathrm{~h}$, and the mixture stirred at $31{ }^{\circ} \mathrm{C}$. The solution turned from orange, then yellow. After addition of the peracid, the temperature was increased to $45^{\circ} \mathrm{C}$, and the mixture stirred for $2 \mathrm{~h}$. The reaction was monitored by ${ }^{1} \mathrm{H}$ NMR spectroscopy and after the signals for SM1 had completely disappeared, the excess AcOOH and $\mathrm{AcOH}$ were removed on a rotary evaporator under vacuum at room temperature to give 
SM2 $(2.31 \mathrm{~g}, 6.08 \mathrm{mmol}, 72 \%)$ as a pale yellow solid. M.p. $98-99{ }^{\circ} \mathrm{C}$ (decomposition). IR (film): $1646,1275 \mathrm{~cm}^{-1} ; \delta_{\mathrm{H}}\left(500 \mathrm{MHz}, \mathrm{CDCl}_{3}\right): 8.15-8.12(1 \mathrm{H}, \mathrm{m}, \mathrm{ArH}), 7.58-7.54(1 \mathrm{H}, \mathrm{m}$, $\operatorname{ArH}),(1 \mathrm{H}, \mathrm{m}, \mathrm{ArH}), 7.14-7.10(1 \mathrm{H}, \mathrm{m}, \mathrm{ArH}), 4.11\left(2 \mathrm{H}, \mathrm{t}, \mathrm{J}=7.0 \mathrm{~Hz}, \mathrm{OCH}_{2} \mathrm{CH}_{2} \mathrm{CH}_{3}\right), 1.97$ $\left(6 \mathrm{H}, \mathrm{s}, \mathrm{C}(\mathrm{O}) \mathrm{CH}_{3}\right), 1.88\left(2 \mathrm{H}\right.$, sextuplet, $\left.J=7.0 \mathrm{~Hz}, \mathrm{OCH}_{2} \mathrm{CH}_{2} \mathrm{CH}_{3}\right), 1.07(3 \mathrm{H}, \mathrm{t}, J=7.0 \mathrm{~Hz}$, $\left.\mathrm{OCH}_{2} \mathrm{CH}_{2} \mathrm{CH}_{3}\right) ; \delta_{\mathrm{C}}\left(125 \mathrm{MHz}, \mathrm{CDCl}_{3}\right): 176.7(\mathrm{C}=\mathrm{O}), 155.8(\mathrm{C}), 137.7(\mathrm{CH}), 134.4(\mathrm{CH})$, $122.6(\mathrm{CH}), 113.7(\mathrm{C}), 112.8(\mathrm{CH}), 71.2\left(\mathrm{CH}_{2}\right), 22.4\left(\mathrm{CH}_{2}\right), 20.4\left(\mathrm{CH}_{3}\right), 10.6\left(\mathrm{CH}_{3}\right)$. Data consistent with published values. ${ }^{1}$

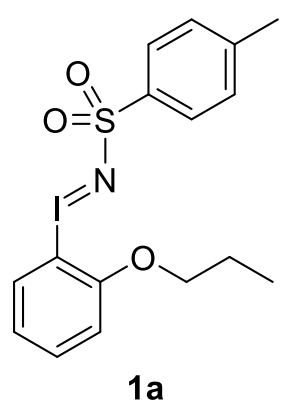

\section{4-Methyl-N-[(2-propoxyphenyl)- $\lambda^{3}$-iodanylidene]benzenesulfonamide} 1a. $\mathrm{KOH}(150 \mathrm{mg}, 2.67 \mathrm{mmol})$ and $\mathrm{TsNH}_{2}(182 \mathrm{mg}, 1.06 \mathrm{mmol})$ were dissolved in $\mathrm{MeOH}(4.1 \mathrm{ml})$, and the solution cooled to $0{ }^{\circ} \mathrm{C} . \mathrm{SM} 2$ (440 $\mathrm{mg}, 1.16 \mathrm{mmol}$ ) was added, and the mixture stirred at $0{ }^{\circ} \mathrm{C}$ for $2.5 \mathrm{~h}$. The resulting yellow solution was allowed to warm to room temperature over a further $30 \mathrm{~min}, \mathrm{H}_{2} \mathrm{O}(27 \mathrm{ml})$ added at which point a pale yellow precipitate was formed. The mixture was cooled to $-78{ }^{\circ} \mathrm{C}$ to induce further precipitation. Upon reaching room temperature the precipitate was filtered off, washed with ${ }^{n}$ hexane $(10 \mathrm{ml})$, and dried in vacuo to give 1a (311 mg, $0.721 \mathrm{mmol}, 68 \%)$ as a pale yellow powder. M.p. $109-110{ }^{\circ} \mathrm{C}$ (decomposition). IR (film): $1579(\mathrm{C}=\mathrm{C}), 1170(\mathrm{~S}=\mathrm{O}) \mathrm{cm}^{-1}$; $\delta_{\mathrm{H}}\left(500 \mathrm{MHz}, \mathrm{CDCl}_{3}\right): 7.83(2 \mathrm{H}, \mathrm{d}$, $J=8.2 \mathrm{~Hz}, \operatorname{Ar} H), 7.66-7.62(1 \mathrm{H}, \mathrm{m}, \operatorname{Ar} H), 7.42-7.38(1 \mathrm{H}, \mathrm{m}, \operatorname{ArH}), 7.18(2 \mathrm{H}, \mathrm{d}, J=8.2 \mathrm{~Hz}$, $\operatorname{ArH}), 7.09-7.04(1 \mathrm{H}, \mathrm{m}, \mathrm{ArH}), 6.90-6.86(1 \mathrm{H}, \mathrm{m}, \operatorname{ArH}), 4.05(2 \mathrm{H}, \mathrm{t}, J=7.1 \mathrm{~Hz}$, $\left.\mathrm{OCH}_{2} \mathrm{CH}_{2} \mathrm{CH}_{3}\right), 2.36\left(3 \mathrm{H}, \mathrm{s}, \mathrm{ArCH}_{3}\right), 1.82\left(2 \mathrm{H}\right.$, sextuplet, $\left.J=7.1 \mathrm{~Hz}, \mathrm{OCH}_{2} \mathrm{CH}_{2} \mathrm{CH}_{3}\right), 1.03$ $\left(3 \mathrm{H}, \mathrm{t}, J=7.1 \mathrm{~Hz}, \mathrm{OCH}_{2} \mathrm{CH}_{2} \mathrm{CH}_{3}\right) ; \delta_{\mathrm{C}}\left(125 \mathrm{MHz}, \mathrm{CDCl}_{3}\right): 154.5(\mathrm{C}), 142.0$ (C), 140.3 (C), $132.4(\mathrm{CH}), 129.4(\mathrm{CH}), 129.3(\mathrm{CH}), 127.0(\mathrm{CH}), 124.1(\mathrm{CH}), 112.3(\mathrm{CH}), 103.3(\mathrm{C}), 71.6$ $\left(\mathrm{CH}_{2}\right), 22.3\left(\mathrm{CH}_{2}\right), 21.4\left(\mathrm{CH}_{3}\right), 10.8\left(\mathrm{CH}_{3}\right)$; HRMS $\left(\mathrm{ES}^{+}\right)$calcd. for $\mathrm{C}_{16} \mathrm{H}_{19} \mathrm{INO}_{3} \mathrm{~S}[\mathrm{MH}]^{+}$: 432.0125, found: 432.0126. Data consistent with published values. ${ }^{1}$ 


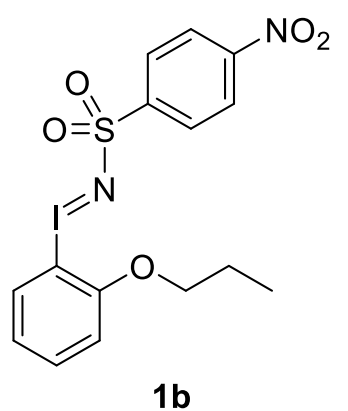

\section{4-Nitro-N-((2-propoxyphenyl)- $\lambda^{3}$-iodanylidene)benzenesulfonamide}

1b. $\mathrm{KOH}$ (150 mg, $2.67 \mathrm{mmol})$ was dissolved in $\mathrm{MeOH}(4.1 \mathrm{ml})$, and 4nitrobenzenesulfonamide $(214 \mathrm{mg}, 1.06 \mathrm{mmol})$ added. The mixture was cooled to $0^{\circ} \mathrm{C}$, and SM2 (440 $\left.\mathrm{mg}, 1.16 \mathrm{mmol}\right)$ was added. The mixture was stirred at $0^{\circ} \mathrm{C}$ for $2.5 \mathrm{~h}$. The resulting yellow mixture was allowed to warm to room temperature over a further $30 \mathrm{~min}, \mathrm{H}_{2} \mathrm{O}(27 \mathrm{ml})$ added, at which point a pale yellow precipitate was formed. The mixture was cooled to $-78{ }^{\circ} \mathrm{C}$ to induce further precipitation. Upon reaching room temperature, the precipitate was filtered off, washed with ${ }^{n}$ hexane $(10 \mathrm{ml})$, and dried in vacuo to give $1 \mathrm{~b}(264 \mathrm{mg}, 0.571 \mathrm{mmol}, 54 \%)$ as a pale yellow powder. M.p. $131-132{ }^{\circ} \mathrm{C}$ (decomposition). IR (film): $3093(\mathrm{C}-\mathrm{H}), 1517,1347$ (S=O), 1121 $(\mathrm{S}=\mathrm{O}) \mathrm{cm}^{-1} ; \delta_{\mathrm{H}}\left(500 \mathrm{MHz}, \mathrm{CDCl}_{3}\right): 8.20(2 \mathrm{H}, \mathrm{d}, J=8.8 \mathrm{~Hz}, \mathrm{ArH}), 8.09(2 \mathrm{H}, \mathrm{d}, J=8.8 \mathrm{~Hz}$, $\operatorname{ArH}), 7.59(1 \mathrm{H}, \mathrm{d}, J=8.0 \mathrm{~Hz}, \operatorname{ArH}), 7.43(1 \mathrm{H}, \mathrm{t}, J=8.0 \mathrm{~Hz}, \operatorname{ArH}), 7.06(1 \mathrm{H}, \mathrm{t}, J=8.0 \mathrm{~Hz}$, $\operatorname{ArH}), 6.90(1 \mathrm{H}, \mathrm{d}, J=8.0 \mathrm{~Hz}, \mathrm{ArH}), 4.06\left(2 \mathrm{H}, \mathrm{t}, J=7.1 \mathrm{~Hz}, \mathrm{OCH}_{2} \mathrm{CH}_{2} \mathrm{CH}_{3}\right), 1.83(2 \mathrm{H}$, sextuplet, $\left.J=7.1 \mathrm{~Hz}, \mathrm{OCH}_{2} \mathrm{CH}_{2} \mathrm{CH}_{3}\right), 1.05\left(3 \mathrm{H}, \mathrm{t}, J=7.1 \mathrm{~Hz}, \mathrm{OCH}_{2} \mathrm{CH}_{2} \mathrm{CH}_{3}\right) ; \delta_{\mathrm{C}}(125 \mathrm{MHz}$, $\left.\mathrm{CDCl}_{3}\right): 154.5(\mathrm{C}), 149.23(\mathrm{C}), 149.20(\mathrm{C}), 133.1(\mathrm{CH}), 129.5(\mathrm{CH}), 128.2(\mathrm{CH}), 124.3(\mathrm{CH})$, $123.9(\mathrm{CH}), 112.6(\mathrm{CH}), 103.0(\mathrm{C}), 71.6\left(\mathrm{CH}_{2}\right), 22.3\left(\mathrm{CH}_{2}\right), 10.5\left(\mathrm{CH}_{3}\right)$; HRMS $\left(\mathrm{ES}^{+}\right)$calcd. for $\mathrm{C}_{15} \mathrm{H}_{15} \mathrm{IN}_{2} \mathrm{NaO}_{5} \mathrm{~S}[\mathrm{MNa}]^{+}:$484.9639, found: 484.9634 .

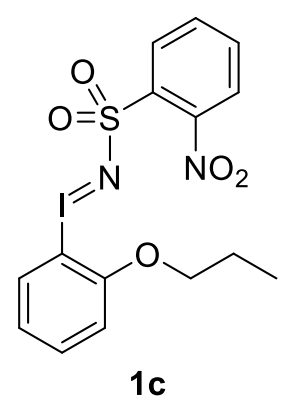

\section{2-Nitro-N-[(2-propoxyphenyl)- $\lambda^{3}$-iodanylidene]benzenesulfonamide $1 \mathrm{c}$.} $\mathrm{KOH}(150 \mathrm{mg}, 2.67 \mathrm{mmol})$ was dissolved in $\mathrm{MeOH}(4.1 \mathrm{ml})$, and 2nitrobenzenesulfonamide $(214 \mathrm{mg}, 1.06 \mathrm{mmol})$ added. The mixture was cooled to $0{ }^{\circ} \mathrm{C}$, and SM2 (440 $\left.\mathrm{mg}, 1.16 \mathrm{mmol}\right)$ was added. The mixture was stirred at $0{ }^{\circ} \mathrm{C}$ for $2.5 \mathrm{~h}$. The resulting yellow mixture was allowed to warm to room temperature over a further $30 \mathrm{~min}, \mathrm{H}_{2} \mathrm{O}(27 \mathrm{ml})$ added, at which point a pale yellow precipitate was formed. The mixture was cooled to $-78{ }^{\circ} \mathrm{C}$ to induce further precipitation. Upon reaching room temperature, the precipitate was filtered off, washed with ${ }^{n}$ hexane (10 $\mathrm{ml}$ ), and dried in vacuo to give $1 \mathrm{c}(284 \mathrm{mg}, 0.614 \mathrm{mmol}, 58 \%)$ as a pale yellow solid. M.p. 
$132-133{ }^{\circ} \mathrm{C}$ (decomposition). IR (film): 3088 (C-H), $2974(\mathrm{C}-\mathrm{H}), 2941(\mathrm{C}-\mathrm{H}), 2882(\mathrm{C}-\mathrm{H})$, 1579, 1541, $1365(\mathrm{~S}=\mathrm{O}) \mathrm{cm}^{-1} ; \delta_{\mathrm{H}}\left(500 \mathrm{MHz}_{\mathrm{CDCl}}\right): 8.24-8.21(1 \mathrm{H}, \mathrm{m}, \mathrm{ArH}), 7.85-7.81$ $(1 \mathrm{H}, \mathrm{m}, \mathrm{ArH}), 7.64-7.55(3 \mathrm{H}, \mathrm{m}, \mathrm{ArH}), 7.47-7.41(1 \mathrm{H}, \mathrm{m}, \mathrm{ArH}), 7.19-7.14(1 \mathrm{H}, \mathrm{m}, \mathrm{ArH})$, $6.95-6.91(1 \mathrm{H}, \mathrm{m}, \mathrm{ArH}), 4.09\left(2 \mathrm{H}, \mathrm{t}, J=7.0 \mathrm{~Hz}, \mathrm{OCH}_{2} \mathrm{CH}_{2} \mathrm{CH}_{3}\right), 1.85(2 \mathrm{H}$, sextuplet, $J=7.0$ $\left.\mathrm{Hz}, \mathrm{OCH}_{2} \mathrm{CH}_{2} \mathrm{CH}_{3}\right), 1.05\left(3 \mathrm{H}, \mathrm{t}, J=7.0 \mathrm{~Hz}, \mathrm{OCH}_{2} \mathrm{CH}_{2} \mathrm{CH}_{3}\right) ; \delta_{\mathrm{C}}\left(125 \mathrm{MHz}, \mathrm{CDCl}_{3}\right): 154.4(\mathrm{C})$, $147.5(\mathrm{C}), 136.8(\mathrm{C}), 132.6(\mathrm{CH}), 132.1(\mathrm{CH}), 131.8(\mathrm{CH}), 130.7(\mathrm{CH}), 129.0(\mathrm{CH}), 124.5$ $(\mathrm{CH}), 123.7(\mathrm{CH}), 112.4(\mathrm{CH}), 104.5(\mathrm{C}), 71.6\left(\mathrm{CH}_{2}\right), 22.3\left(\mathrm{CH}_{2}\right), 10.5\left(\mathrm{CH}_{3}\right)$; HRMS $\left(\mathrm{ES}{ }^{+}\right)$ calcd. for $\mathrm{C}_{15} \mathrm{H}_{15} \mathrm{IN}_{2} \mathrm{NaO}_{5} \mathrm{~S}[\mathrm{MNa}]^{+}: 484.9639$, found: 484.9639 .

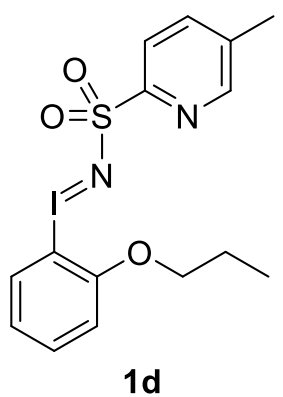

\section{5-Methyl-N-[(2-propoxyphenyl)- $\lambda^{3}$-iodanylidene]pyridine-2-sulfon}

amide 1d. $\mathrm{KOH}$ (150 mg, $2.67 \mathrm{mmol}$ ) and 5-methylpyridine-2-sulfonamide (183 $\mathrm{mg}, 1.06 \mathrm{mmol})$ were dissolved in $\mathrm{MeOH}(4.1 \mathrm{ml})$, and the solution cooled to $0{ }^{\circ} \mathrm{C}$. SM2 (440 mg, $\left.1.16 \mathrm{mmol}, 1.09 \mathrm{eq}\right)$ was added, and the mixture stirred at $0{ }^{\circ} \mathrm{C}$ for $2.5 \mathrm{~h}$. The resulting yellow solution was allowed to warm to room temperature over a further $30 \mathrm{~min}, \mathrm{H}_{2} \mathrm{O}(27 \mathrm{ml})$ added, at which point a pale yellow precipitate was formed. The mixture was cooled to $-78{ }^{\circ} \mathrm{C}$ to induce further precipitation. Upon reaching room temperature, the precipitate was filtered off, washed with ${ }^{n}$ hexane $(10 \mathrm{ml})$, and dried in vacuo to give $1 \mathrm{~d}(280 \mathrm{mg}, 0.648 \mathrm{mmol}, 61 \%)$ as a pale yellow solid. M.p. $127-128{ }^{\circ} \mathrm{C}$ (decomposition). IR (film): 1585, 1460, $1152(\mathrm{~S}=\mathrm{O}) \mathrm{cm}^{-1} ; \delta_{\mathrm{H}}(500$ $\left.\mathrm{MHz}, \mathrm{CDCl}_{3}\right): 8.40(1 \mathrm{H}, \mathrm{s}, \operatorname{Ar} H), 8.00-7.96(1 \mathrm{H}, \mathrm{m}, \operatorname{Ar} H), 7.93(1 \mathrm{H}, \mathrm{d}, J=8.0 \mathrm{~Hz}, \operatorname{ArH}), 7.70$ - $7.65(1 \mathrm{H}, \mathrm{m}, \mathrm{ArH}), 7.46-7.40(1 \mathrm{H}, \mathrm{m}, \mathrm{ArH}), 7.25-7.20(1 \mathrm{H}, \mathrm{m}, \operatorname{ArH}), 6.95-6.91(1 \mathrm{H}, \mathrm{m}$, $\operatorname{ArH}), 4.10\left(2 \mathrm{H}, \mathrm{t}, J=7.0 \mathrm{~Hz}, \mathrm{OCH}_{2} \mathrm{CH}_{2} \mathrm{CH}_{3}\right), 2.41\left(3 \mathrm{H}, \mathrm{s}, \mathrm{ArCH}_{3}\right), 1.86(2 \mathrm{H}$, sextuplet, $J=$ $\left.7.0 \mathrm{~Hz}, \mathrm{OCH}_{2} \mathrm{CH}_{2} \mathrm{CH}_{3}\right), 1.07\left(3 \mathrm{H}, J=7.0 \mathrm{~Hz}, \mathrm{OCH}_{2} \mathrm{CH}_{2} \mathrm{CH}_{3}\right) ; \delta_{\mathrm{C}}\left(125 \mathrm{MHz}, \mathrm{CDCl}_{3}\right): 158.9$ (C), $154.5(\mathrm{C}), 148.2(\mathrm{CH}), 138.6(\mathrm{CH}), 135.5(\mathrm{C}), 132.0(\mathrm{CH}), 128.6(\mathrm{CH}), 124.2(\mathrm{CH})$, $119.1(\mathrm{CH}), 112.0(\mathrm{CH}), 105.7(\mathrm{C}), 71.3\left(\mathrm{CH}_{2}\right), 22.3\left(\mathrm{CH}_{2}\right), 18.4\left(\mathrm{CH}_{3}\right), 10.6\left(\mathrm{CH}_{3}\right)$; HRMS $\left(E S^{+}\right)$calcd. for $\mathrm{C}_{15} \mathrm{H}_{17} \mathrm{IN}_{2} \mathrm{NaO}_{3} \mathrm{~S}$ [MNa] ${ }^{+}$: 454.9897, found: 454.9899 . 


\section{Optimization of conditions for aziridination}

Copper catalyzed aziridination of alkenes with $\mathrm{Phl}=\mathrm{NT}$ are known to occur faster in polar aprotic solvents such as $\mathrm{MeCN}$ or $\mathrm{MeNO}_{2}{ }^{2}$ The procedure reported by Yoshimura et al. was performed using $\mathrm{Cu}(\mathrm{OTf})_{2}$ in a minimum volume of acetonitrile. ${ }^{1}$ However, this was found to be unsuitable for adapting to flow, as 1 a has low solubility in acetonitrile. Trial reactions were run in NMR tubes under a variety of conditions (Table 1), and the conversion monitored by ${ }^{1} \mathrm{H}$ NMR. It was found that while the aziridination did not work in the absence of acetonitrile, 1a could first be dissolved in $\mathrm{CH}_{2} \mathrm{Cl}_{2}$ before adding to a solution of styrene and catalyst in $\mathrm{CD}_{3} \mathrm{CN}$. In the absence of catalyst, the aziridination did not work (entry 1); instead, 1a decomposed to give the sulfonamide at about $10 \%$ per hour. 0.1 equivalents of $\mathrm{Cu}(\mathrm{OTf})_{2}$ catalyzed the aziridination of styrene with 1 a efficiently (entry 2 ), but (pyr) ${ }_{4} \mathrm{Cu}(\mathrm{OTf})_{2}$ was even better (entry 3).

Table 1. Optimization of conditions for aziridination of styrene by $1 \mathbf{a}$.

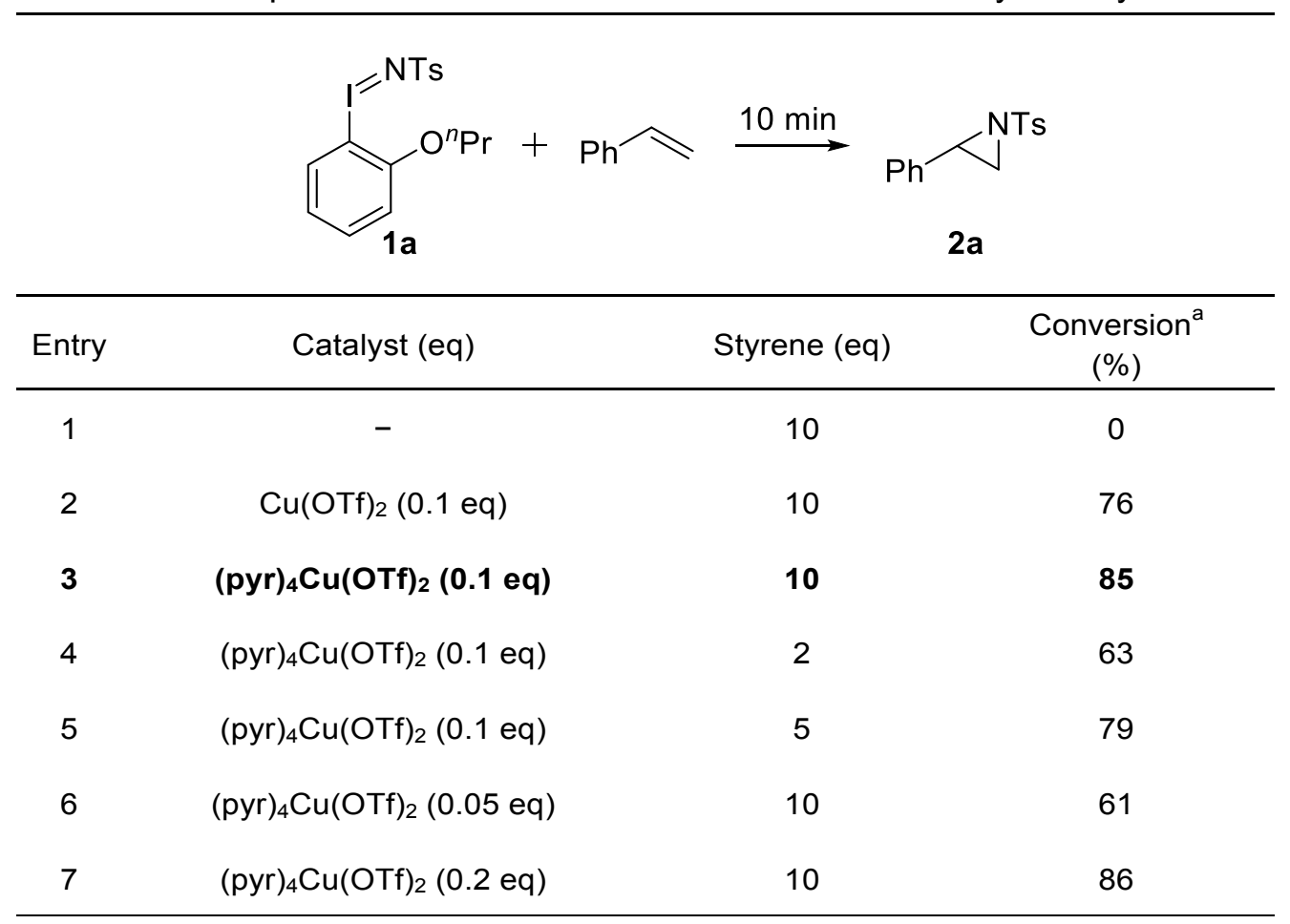

\footnotetext{
${ }^{a}$ Catalyst and styrene were dissolved in $\mathrm{CD}_{3} \mathrm{CN} ; 1$ eq of 1 a was dissolved in $\mathrm{CH}_{2} \mathrm{Cl}_{2}$; the two solutions were mixed. Conversion was determined after $10 \mathrm{~min}$ by ${ }^{1} \mathrm{H}$ NMR spectroscopy.
} 


\section{Flow synthesis of aziridines $2 a-I$}

A custom flow system was assembled by

Figure S1. Flow reactor used; A and B were used, C blocked off, connecting computer controlled Tricontinent and the tubing connected added to the total reaction volume. C3000 syringe pumps to a XXL-S-01 threeinput flow reactor purchased from Little Things Factory. As only two inputs A and B were used, the third input $C$ was blocked off; the reactor had a total reaction volume of $4.5 \mathrm{ml}$ (including tubing carrying away solution) when used in this way.

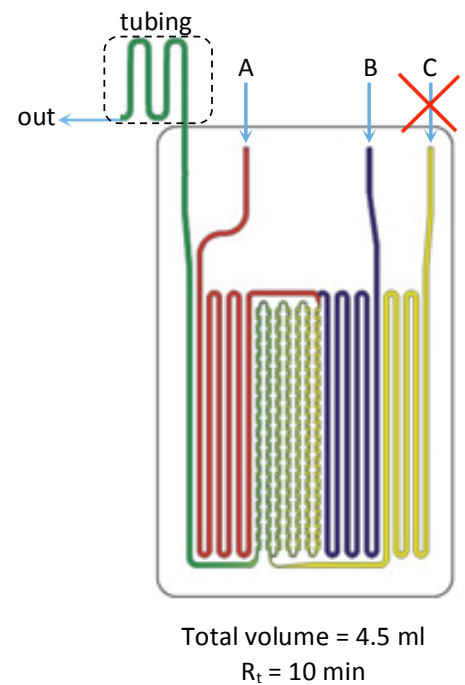

$\mathrm{Arl}=\mathrm{NSO}_{2} \mathrm{R}(0.10 \mathrm{mmol}, 1.0 \mathrm{eq})$ was dissolved in $\mathrm{CH}_{2} \mathrm{Cl}_{2}(2.0 \mathrm{ml})$ to give solution $\mathrm{A}$; vigorous shaking was sometimes necessary for complete dissolution. Alkene $(1.00 \mathrm{mmol}, 10 \mathrm{eq}$ except for cycloheptene where $2.00 \mathrm{mmol}, 20$ eq was used) and $(\mathrm{pyr})_{4} \mathrm{Cu}(\mathrm{OTf})_{2}(0.01 \mathrm{mmol}$, $0.1 \mathrm{eq})$ were dissolved in anhydrous $\mathrm{MeCN}(2.0 \mathrm{ml})$ to give solution $\mathrm{B}$. Solutions $\mathrm{A}$ and $\mathrm{B}$ were combined in the XXL-S-01 microreactor at room temperature, with a residence time of $10 \mathrm{~min}$. The reaction was stopped by cooling the outlet stream to $-78^{\circ} \mathrm{C}$. Upon completion of the run, additional quantities of $\mathrm{CH}_{2} \mathrm{Cl}_{2}$ and $\mathrm{MeCN}$ were passed through the microreactor at $A$ and $B$ respectively to ensure all the product was collected. The resulting mixture was concentrated under vacuum and purified by gradient column chromatography $(5: 1$ ${ }^{n}$ hexane:EtOAc, then $3: 1{ }^{n}$ hexane:EtOAc) to give the following products: 


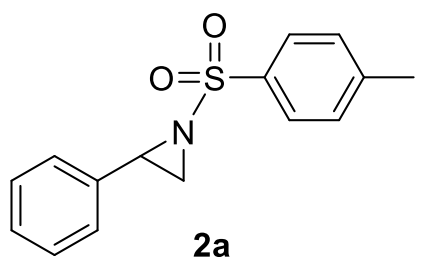

2-Phenyl-1-tosylaziridine 2a. Using 1a (44 mg, $0.102 \mathrm{mmol})$, styrene $(105 \mathrm{mg}, 1.01 \mathrm{mmol})$ and $(\mathrm{pyr})_{4} \mathrm{Cu}(\mathrm{OTf})_{2}(7 \mathrm{mg}, 0.010$ $\mathrm{mmol}), 2 \mathrm{a}$ (23 $\mathrm{mg}, 0.084 \mathrm{mmol}, 82 \%)$ was produced as a clear oil. IR (film): $1596(\mathrm{C}=\mathrm{C}), 1323(\mathrm{~S}=\mathrm{O}), 1160(\mathrm{~S}=\mathrm{O}) \mathrm{cm}^{-1} ; \delta_{\mathrm{H}}(500$ $\left.\mathrm{MHz}, \mathrm{CDCl}_{3}\right): 7.87(2 \mathrm{H}, \mathrm{d}, J=8.3 \mathrm{~Hz}, \mathrm{ArH}), 7.33(2 \mathrm{H}, \mathrm{d}, J=8.3 \mathrm{~Hz}, \mathrm{ArH}), 7.32-7.27(3 \mathrm{H}$, m, ArH), $7.24-7.20(2 \mathrm{H}, \mathrm{m}, \operatorname{ArH}), 3.78(1 \mathrm{H}, \mathrm{dd}, J=4.5,7.2 \mathrm{~Hz}, \mathrm{PhCH}), 2.99(1 \mathrm{H}, \mathrm{d}, J=7.2$ $\mathrm{Hz}, \mathrm{CH} H), 2.43\left(3 \mathrm{H}, \mathrm{s}, \mathrm{ArCH}_{3}\right), 2.39(1 \mathrm{H}, \mathrm{d}, J=4.5 \mathrm{~Hz}, \mathrm{CHH}) ; \delta_{\mathrm{C}}\left(125 \mathrm{MHz}, \mathrm{CDCl}_{3}\right): 144.7$ (C), $135.1(\mathrm{C}), 135.0(\mathrm{C}), 129.8(\mathrm{CH}), 128.6(\mathrm{CH}), 128.3(\mathrm{CH}), 128.0(\mathrm{CH}), 126.6(\mathrm{CH}), 41.1$ (CH), $36.0\left(\mathrm{CH}_{2}\right), 21.7\left(\mathrm{CH}_{3}\right) ; \mathrm{m} / \mathrm{z}\left(\mathrm{ES}^{+}\right) 296$ [MNa] ${ }^{+}$; HRMS $\left(\mathrm{ES}^{+}\right)$calcd. for $\mathrm{C}_{15} \mathrm{H}_{15} \mathrm{NNaO}_{2} \mathrm{~S}$ $\left[\mathrm{MNa}^{+}:\right.$296.0716, found: 296.0718 . Data consistent with published values. ${ }^{1}$

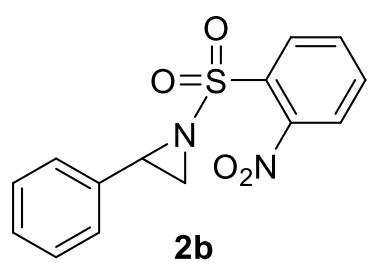

1-[(2-Nitrophenyl)sulfonyl]-2-phenylaziridine 2b. Using 1c (47 $\mathrm{mg}, 0.102 \mathrm{mmol})$, styrene (105 mg, $1.01 \mathrm{mmol})$ and $(\mathrm{pyr})_{4} \mathrm{Cu}(\mathrm{OTf})_{2}$ ( $7 \mathrm{mg}, 0.010 \mathrm{mmol}), \mathbf{2 b}(24 \mathrm{mg}, 0.079 \mathrm{mmol}, 77 \%)$ was produced as a clear oil. IR (film): $1543(\mathrm{C}=\mathrm{C}), 1336(\mathrm{~S}=\mathrm{O}), 1167(\mathrm{~S}=\mathrm{O}) \mathrm{cm}^{-1} ; \delta_{\mathrm{H}}$ $\left(500 \mathrm{MHz}, \mathrm{CDCl}_{3}\right): 8.26-8.23(1 \mathrm{H}, \mathrm{m}, \mathrm{ArH}), 7.78-7.72(3 \mathrm{H}, \mathrm{m}, \mathrm{ArH}), 7.36-7.29(5 \mathrm{H}, \mathrm{m}$, $\operatorname{ArH}), 4.04(1 \mathrm{H}, \mathrm{dd}, J=4.8,7.2 \mathrm{~Hz}, \operatorname{ArCH}), 3.25(1 \mathrm{H}, \mathrm{d}, J=7.2 \mathrm{~Hz}, \mathrm{CH} H), 2.63(1 \mathrm{H}, \mathrm{d}, J=$ $4.8 \mathrm{~Hz}, \mathrm{CHH}) ; \delta_{\mathrm{C}}\left(125 \mathrm{MHz}, \mathrm{CDCl}_{3}\right): 148.6(\mathrm{C}), 134.7(\mathrm{C}), 134.5(\mathrm{CH}), 132.2(\mathrm{CH}), 132.1$ (C), $131.3(\mathrm{CH}), 128.7(\mathrm{CH}), 128.6(\mathrm{CH}), 126.6(\mathrm{CH}), 124.5(\mathrm{CH}), 42.9(\mathrm{CH}), 38.1\left(\mathrm{CH}_{2}\right) ; \mathrm{m} / \mathrm{z}$ $\left(\mathrm{ES}^{+}\right) 327[\mathrm{MNa}]^{+}$; HRMS $\left(\mathrm{ES}^{+}\right)$calcd. for $\mathrm{C}_{14} \mathrm{H}_{12} \mathrm{~N}_{2} \mathrm{NaO}_{4} \mathrm{~S}$ [MNa] $]^{+}: 327.0410$, found: 327.0406. Data consistent with published values. ${ }^{3}$

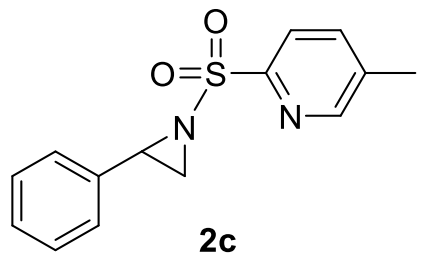

5-Methyl-2-[(2-phenylaziridin-1-yl)sulfonyl]pyridine 2c. Using 1d (44 mg, mmol, $0.102 \mathrm{mmol})$, styrene (105 mg, $1.01 \mathrm{mmol})$ and $(\text { pyr })_{4} \mathrm{Cu}(\mathrm{OTf})_{2}(7 \mathrm{mg}, 0.010 \mathrm{mmol}), 2 \mathrm{c}(22 \mathrm{mg}, 0.080 \mathrm{mmol}, 79 \%)$ was produced as a clear oil. IR (film): $1572(\mathrm{C}=\mathrm{C}), 1327(\mathrm{~S}=\mathrm{O})$, $1171(\mathrm{~S}=\mathrm{O}) \mathrm{cm}^{-1} ; \delta_{\mathrm{H}}\left(500 \mathrm{MHz}, \mathrm{CDCl}_{3}\right): 8.57-8.55(1 \mathrm{H}, \mathrm{m}, \mathrm{Ar} H), 8.02(1 \mathrm{H}, \mathrm{d}, J=8.0 \mathrm{~Hz}$ $\operatorname{ArH}), 7.72(1 \mathrm{H}, \mathrm{d}, J=8.0 \mathrm{~Hz}, \operatorname{ArH}), 7.32-7.24(5 \mathrm{H}, \mathrm{m}, \operatorname{ArH}), 4.00(1 \mathrm{H}, \mathrm{dd}, J=4.7,7.2 \mathrm{~Hz}$, 
$\mathrm{PhCH}), 3.20(1 \mathrm{H}, \mathrm{d}, J=7.2 \mathrm{~Hz}, \mathrm{CHH}), 2.51(1 \mathrm{H}, \mathrm{d}, J=4.7 \mathrm{~Hz}, \mathrm{CHH}), 2.44\left(3 \mathrm{H}, \mathrm{s}, \mathrm{ArCH}_{3}\right) ; \delta_{\mathrm{C}}$ (125 MHz, $\left.\mathrm{CDCl}_{3}\right): 153.3(\mathrm{C}), 150.8(\mathrm{CH}), 138.3(\mathrm{C}), 138.2(\mathrm{CH}), 134.9(\mathrm{C}), 128.5(\mathrm{CH})$, $128.4(\mathrm{CH}), 126.7(\mathrm{CH}), 122.8(\mathrm{CH}), 41.4(\mathrm{CH}), 36.0\left(\mathrm{CH}_{2}\right), 18.6\left(\mathrm{CH}_{3}\right) ; \mathrm{m} / \mathrm{z}\left(\mathrm{ES}^{+}\right) 297$ [MNa] $]^{+}$; HRMS $\left(\mathrm{ES}^{+}\right.$) calcd. for $\mathrm{C}_{14} \mathrm{H}_{14} \mathrm{~N}_{2} \mathrm{NaO}_{2} \mathrm{~S}$ [MNa] ${ }^{+}:$297.0668, found: 297.0670. Data consistent with published values. ${ }^{4}$

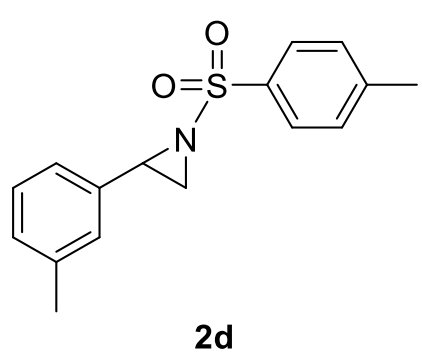

2-(m-Tolyl)-1-tosylaziridine 2d. Using 1a (44 mg, $0.102 \mathrm{mmol})$, 3-methylstyrene $(119 \mathrm{mg}, 1.01 \mathrm{mmol})$ and $(\mathrm{pyr})_{4} \mathrm{Cu}(\mathrm{OTf})_{2}(7 \mathrm{mg}$, $0.010 \mathrm{mmol}), 2 \mathrm{~d}(20 \mathrm{mg}, 0.070 \mathrm{mmol}, 68 \%)$ was produced as a clear oil. IR (film): 1597 (C=C), 1325 (S=O), $1161(\mathrm{~S}=\mathrm{O}) \mathrm{cm}^{-1} ; \delta_{\mathrm{H}}$ $\left(500 \mathrm{MHz}, \mathrm{CDCl}_{3}\right): 7.87(2 \mathrm{H}, \mathrm{d}, J=8.3 \mathrm{~Hz}, \operatorname{ArH}), 7.33(2 \mathrm{H}, \mathrm{d}, J=$ 8.3 Hz, ArH), $7.18(1 \mathrm{H}, \mathrm{t}, J=7.9 \mathrm{~Hz}, \operatorname{ArH}), 7.08(1 \mathrm{H}, \mathrm{d}, J=7.9 \mathrm{~Hz}, \operatorname{ArH}), 7.03-6.99(2 \mathrm{H}, \mathrm{m}$, $\operatorname{ArH}), 3.74(1 \mathrm{H}, \mathrm{dd}, J=4.5,7.2 \mathrm{~Hz}, \operatorname{ArCH}), 2.96(1 \mathrm{H}, \mathrm{d}, J=7.2 \mathrm{~Hz}, \mathrm{CHH}), 2.43(3 \mathrm{H}, \mathrm{s}$, $\left.\mathrm{ArCH}_{3}\right), 2.38(1 \mathrm{H}, \mathrm{d}, J=4.5 \mathrm{~Hz}, \mathrm{CHH}), 2.30\left(3 \mathrm{H}, \mathrm{s}, \mathrm{ArCH}_{3}\right) ; \delta_{\mathrm{C}}\left(125 \mathrm{MHz}, \mathrm{CDCl}_{3}\right): 144.6(\mathrm{C})$, $138.3(\mathrm{C}), 135.04(\mathrm{C}), 134.97(\mathrm{C}), 129.8(\mathrm{CH}), 129.1(\mathrm{CH}), 128.5(\mathrm{CH}), 128.0(\mathrm{CH}), 127.2$ $(\mathrm{CH}), 123.7(\mathrm{CH}), 41.1(\mathrm{CH}), 35.9\left(\mathrm{CH}_{2}\right), 21.7\left(\mathrm{CH}_{3}\right), 21.3\left(\mathrm{CH}_{3}\right) ; \mathrm{m} / \mathrm{z}\left(\mathrm{ES}^{+}\right) 310[\mathrm{MNa}]^{+}$; HRMS (ES ${ }^{+}$) calcd. for $\mathrm{C}_{16} \mathrm{H}_{17} \mathrm{NNaO}_{2} \mathrm{~S}[\mathrm{MNa}]^{+}:$310.0872, found: 310.0867 . Data consistent with published values. ${ }^{5}$

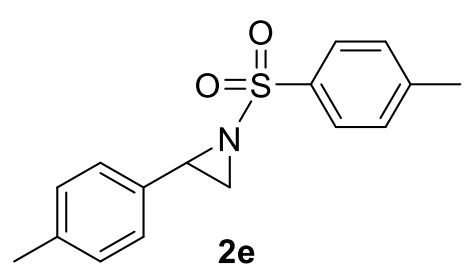

2-(p-Tolyl)-1-tosylaziridine 2e. Using 1a (44 mg, 0.102 mmol), 4-methylstyrene $(119 \mathrm{mg}, \quad 1.01 \mathrm{mmol})$ and $(\text { pyr })_{4} \mathrm{Cu}(\mathrm{OTf})_{2}(7 \mathrm{mg}, 0.010 \mathrm{mmol}), 2 \mathrm{e}(20 \mathrm{mg}, 0.070 \mathrm{mmol}$, $68 \%$ ) was produced as a clear oil. IR (film): $1597(\mathrm{C}=\mathrm{C}), 1323$ $(\mathrm{S}=\mathrm{O}), 1161(\mathrm{~S}=\mathrm{O}) \mathrm{cm}^{-1} ; \delta_{\mathrm{H}}\left(500 \mathrm{MHz}, \mathrm{CDCl}_{3}\right): 7.88(2 \mathrm{H}, \mathrm{d}, J=8.2 \mathrm{~Hz}, \operatorname{ArH}), 7.35(2 \mathrm{H}, \mathrm{d}, J$ $=8.2 \mathrm{~Hz}, \operatorname{ArH}), 7.13-7.11(4 \mathrm{H}, \mathrm{m}, \operatorname{ArH}), 3.76(1 \mathrm{H}, \mathrm{dd}, J=4.5,7.2 \mathrm{~Hz}, \operatorname{ArCH}), 2.99(1 \mathrm{H}, \mathrm{d}, J$ $=7.2 \mathrm{~Hz}, \mathrm{CHH}), 2.45\left(3 \mathrm{H}, \mathrm{s}, \mathrm{ArCH}_{3}\right), 2.40(1 \mathrm{H}, \mathrm{d}, J=4.5 \mathrm{~Hz}, \mathrm{CHH}), 2.33\left(3 \mathrm{H}, \mathrm{s}, \mathrm{ArCH}_{3}\right) ; \delta_{\mathrm{C}}$ (125 MHz, CDCl $)$ : 144.6 (C), 138.2 (C), 135.1 (C), 132.0 (C), $129.7(\mathrm{CH}), 129.3(\mathrm{CH}), 127.9$ $(\mathrm{CH}), 126.5(\mathrm{CH}), 41.1(\mathrm{CH}), 35.8\left(\mathrm{CH}_{2}\right), 21.7\left(\mathrm{CH}_{3}\right), 21.2\left(\mathrm{CH}_{3}\right) ; \mathrm{m} / \mathrm{z}\left(\mathrm{ES}^{+}\right) 310[\mathrm{MNa}]^{+}$; 
HRMS $\left(\mathrm{ES}^{+}\right)$calcd. for $\mathrm{C}_{16} \mathrm{H}_{17} \mathrm{NNaO}_{2} \mathrm{~S}$ [MNa] ${ }^{+}: 310.0872$, found: 310.0875. Data consistent with published values. ${ }^{5}$

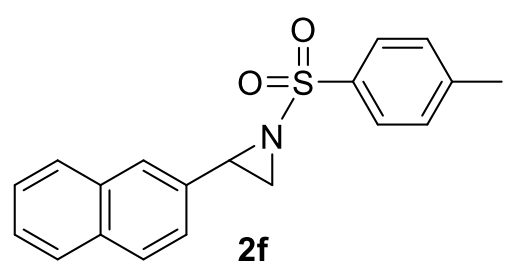

2-(Naphthalen-2-yl)-1-tosylaziridine 2f. Using 1a (44 mg, $0.102 \mathrm{mmol}), 2$-vinylnaphthalene $(155 \mathrm{mg}, 1.01 \mathrm{mmol})$ and $(\text { pyr })_{4} \mathrm{Cu}(\mathrm{OTf})_{2}(7 \mathrm{mg}, 0.010 \mathrm{mmol}), 2 \mathrm{f}(23 \mathrm{mg}, 0.071 \mathrm{mmol}$, $70 \%)$ was produced as a clear oil. IR (film): $1597(\mathrm{C}=\mathrm{C})$, $1324(\mathrm{~S}=\mathrm{O}), 1161(\mathrm{~S}=\mathrm{O}) \mathrm{cm}^{-1} ; \delta_{\mathrm{H}}\left(500 \mathrm{MHz}, \mathrm{CD}_{2} \mathrm{Cl}_{2}\right): 7.91(2 \mathrm{H}, \mathrm{d}, \mathrm{J}=8.1 \mathrm{~Hz}, \mathrm{ArH}), 7.87-$ $7.82(3 \mathrm{H}, \mathrm{m}, \mathrm{ArH}), 7.78(1 \mathrm{H}, \mathrm{s}, \mathrm{ArH}), 7.55-7.49(2 \mathrm{H}, \mathrm{m}, \operatorname{ArH}), 7.41(2 \mathrm{H}, \mathrm{d}, J=8.1 \mathrm{~Hz}, \operatorname{ArH})$, $7.34-7.30(1 \mathrm{H}, \mathrm{m}, \operatorname{ArH}), 3.93(1 \mathrm{H}, \mathrm{dd}, J=4.5,7.2 \mathrm{~Hz}, \operatorname{ArCH}), 3.06(1 \mathrm{H}, \mathrm{d}, J=7.2 \mathrm{~Hz}$, $\mathrm{CHH}), 2.56(1 \mathrm{H}, \mathrm{d}, J=4.5 \mathrm{~Hz}, \mathrm{CHH}), 2.47\left(3 \mathrm{H}, \mathrm{s}, \mathrm{ArCH}_{3}\right) ; \delta_{\mathrm{C}}\left(125 \mathrm{MHz}, \mathrm{CD}_{2} \mathrm{Cl}_{2}\right): 145.0(\mathrm{C})$, $134.9(\mathrm{C}), 133.2(\mathrm{C}), 133.1(\mathrm{C}), 132.6(\mathrm{C}), 129.8(\mathrm{CH}), 128.4(\mathrm{CH}), 127.9(\mathrm{CH}), 127.7(\mathrm{CH})$, $127.6(\mathrm{CH}), 126.5(\mathrm{CH}), 126.3(\mathrm{CH}), 126.1(\mathrm{CH}), 123.9(\mathrm{CH}), 41.1(\mathrm{CH}), 36.0\left(\mathrm{CH}_{2}\right), 21.4$ $\left(\mathrm{CH}_{3}\right) ; \mathrm{m} / \mathrm{z}\left(\mathrm{ES}^{+}\right) 346[\mathrm{MNa}]^{+} ; \mathrm{HRMS}\left(\mathrm{ES}^{+}\right)$calcd. for $\mathrm{C}_{19} \mathrm{H}_{17} \mathrm{NNaO}_{2} \mathrm{~S}[\mathrm{MNa}]^{+}: 346.0872$, found: 346.0875 . Data consistent with published values. ${ }^{5}$

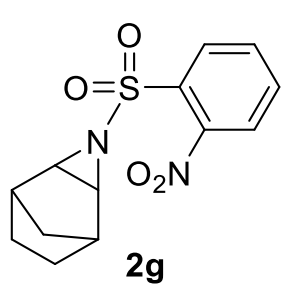

\section{3-[(2-Nitrophenyl)sulfonyl]-3-azatricyclo[3.2.1.0,}

Using 1c (47 mg, $0.102 \mathrm{mmol})$, norbornene (95 mg, $1.01 \mathrm{mmol}$ ) and $(\text { pyr })_{4} \mathrm{Cu}(\mathrm{OTf})_{2}(7 \mathrm{mg}, 0.010 \mathrm{mmol}), 2 \mathrm{~g}(21 \mathrm{mg}, 0.071 \mathrm{mmol}, 70 \%)$ was produced as a clear oil. IR (film): $2974(\mathrm{C}-\mathrm{H}), 2877(\mathrm{C}-\mathrm{H}), 1544(\mathrm{C}=\mathrm{C})$, $1330(\mathrm{~S}=\mathrm{O}), 1123(\mathrm{~S}=\mathrm{O}) \mathrm{cm}^{-1} ; \delta_{\mathrm{H}}\left(500 \mathrm{MHz}, \mathrm{CDCl}_{3}\right): 8.19-8.14(1 \mathrm{H}, \mathrm{m}, \mathrm{ArH}), 7.76-7.70$ $(3 \mathrm{H}, \mathrm{m}, \mathrm{ArH}), 3.22-3.21(2 \mathrm{H}, \mathrm{m}, \mathrm{NCH}), 2.57-2.54\left(2 \mathrm{H}, \mathrm{m}, \mathrm{RR}^{\prime} \mathrm{R}^{\prime \prime} \mathrm{CH}\right), 1.55-1.50(2 \mathrm{H}, \mathrm{m}$, $\left.\mathrm{CH}_{2}\right), 1.44-1.39\left(1 \mathrm{H}, \mathrm{m}, \mathrm{R}_{2} \mathrm{CH} H\right), 1.31-1.26\left(2 \mathrm{H}, \mathrm{m}, \mathrm{CH}_{2}\right), 0.84-0.79\left(1 \mathrm{H}, \mathrm{m}, \mathrm{R}_{2} \mathrm{CH}\right)$; $\delta_{\mathrm{C}}\left(125 \mathrm{MHz}, \mathrm{CDCl}_{3}\right): 148.4(\mathrm{C}), 134.0(\mathrm{CH}), 132.9(\mathrm{C}), 132.0(\mathrm{CH}), 130.5(\mathrm{CH}), 124.3(\mathrm{CH})$, $44.3(\mathrm{CH}), 36.1(\mathrm{CH}), 28.2\left(\mathrm{CH}_{2}\right), 25.4\left(\mathrm{CH}_{2}\right) ; \mathrm{m} / \mathrm{z}\left(\mathrm{ES}^{+}\right) 317[\mathrm{MNa}]^{+}$; HRMS (ES $\left.{ }^{+}\right)$calcd. for $\mathrm{C}_{13} \mathrm{H}_{14} \mathrm{~N}_{2} \mathrm{NaO}_{4} \mathrm{~S}[\mathrm{MNa}]^{+}:$317.0566, found: 317.0555 . 


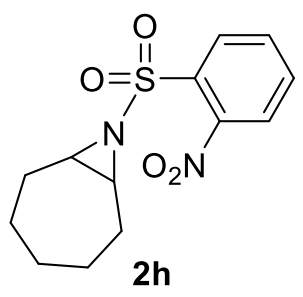

8-[(2-Nitrophenyl)sulfonyl]-8-azabicyclo[5.1.0]octane 2h. Using 1c (47 mg, $0.102 \mathrm{mmol})$, cycloheptene (192 $\mathrm{mg}, 2.00 \mathrm{mmol})$ and $(\text { pyr })_{4} \mathrm{Cu}(\mathrm{OTf})_{2}(7 \mathrm{mg}, 0.010 \mathrm{mmol}), 2 \mathrm{~h}(14 \mathrm{mg}, 0.047 \mathrm{mmol}, 46 \%)$ was produced as a clear oil. IR (film): $2924(\mathrm{C}-\mathrm{H}), 2849(\mathrm{C}-\mathrm{H}), 1592(\mathrm{C}=\mathrm{C})$, $1329(\mathrm{~S}=\mathrm{O}), 1161(\mathrm{~S}=\mathrm{O}) \mathrm{cm}^{-1} ; \delta_{\mathrm{H}}\left(500 \mathrm{MHz}, \mathrm{CDCl}_{3}\right): 8.24-8.20(1 \mathrm{H}, \mathrm{m}, \mathrm{ArH}), 7.78-7.71$ $(3 \mathrm{H}, \mathrm{m}, \mathrm{ArH}), 3.28-3.22(2 \mathrm{H}, \mathrm{m}, \mathrm{NCH}), 2.05-1.88\left(4 \mathrm{H}, \mathrm{m}, \mathrm{CH}_{2}\right), 1.62-1.41\left(5 \mathrm{H}, \mathrm{m}, \mathrm{CH}_{2}\right)$, $1.26-1.16\left(1 \mathrm{H}, \mathrm{m}, \mathrm{CH}_{2}\right) ; \delta_{\mathrm{C}}\left(125 \mathrm{MHz}, \mathrm{CDCl}_{3}\right): 148.5(\mathrm{C}), 134.0(\mathrm{CH}), 133.0(\mathrm{C}), 132.2$ $(\mathrm{CH}), 131.0(\mathrm{CH}), 124.4(\mathrm{CH}), 46.9(\mathrm{CH}), 31.0\left(\mathrm{CH}_{2}\right), 28.2\left(\mathrm{CH}_{2}\right), 25.1\left(\mathrm{CH}_{2}\right) ; \mathrm{m} / \mathrm{z}\left(\mathrm{ES}^{+}\right) 319$ [MNa] $]^{+}$HRMS (ES ${ }^{+}$) calcd. for $\mathrm{C}_{13} \mathrm{H}_{16} \mathrm{~N}_{2} \mathrm{NaO}_{4} \mathrm{~S}$ [MNa] $]^{+}: 319.0723$, found: 319.0729 .

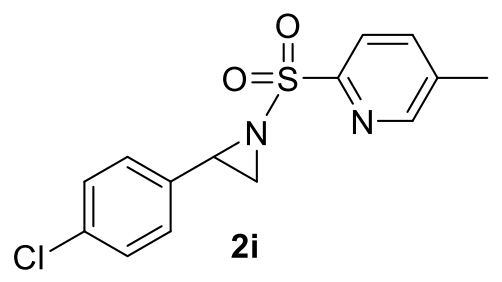

\section{2-[[2-(4-chlorophenyl)aziridin-1-yl]sulfonyl]-5-methyl pyridine}

2i. Using 1d (44 mg, $0.102 \mathrm{mmol})$, 4-chlorostyrene (139 mg, 1.00 $\mathrm{mmol})$ and $(\mathrm{pyr})_{4} \mathrm{Cu}(\mathrm{OTf})_{2}(7 \mathrm{mg}, 0.010 \mathrm{mmol}), 2 \mathbf{i}(24 \mathrm{mg}, 0.078$ mmol, $76 \%$ ) was produced as a clear oil. IR (film): $1572(\mathrm{C}=\mathrm{C})$,

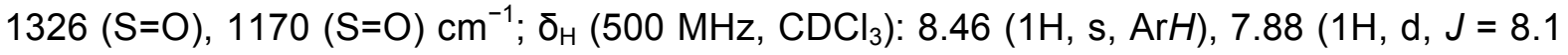
$\mathrm{Hz}, \operatorname{ArH}), 7.66(1 \mathrm{H}, \mathrm{d}, J=8.1 \mathrm{~Hz}, \operatorname{ArH}), 7.21(2 \mathrm{H}, \mathrm{d}, J=8.5 \mathrm{~Hz}, \operatorname{ArH}), 7.13(2 \mathrm{H}, \mathrm{d}, J=8.5$ $\mathrm{Hz}, \operatorname{ArH}), 3.83(1 \mathrm{H}, \mathrm{dd}, J=4.7,7.1 \mathrm{~Hz}, \mathrm{ArCH}), 3.04(1 \mathrm{H}, \mathrm{d}, J=7.1 \mathrm{~Hz}, \mathrm{CH} H), 2.39(1 \mathrm{H}, \mathrm{d}, J$ $=4.7 \mathrm{~Hz}, \mathrm{CHH}), 2.35\left(3 \mathrm{H}, \mathrm{s}, \mathrm{ArCH}_{3}\right) ; \delta_{\mathrm{C}}\left(125 \mathrm{MHz}, \mathrm{CDCl}_{3}\right): 153.4(\mathrm{C}), 151.3(\mathrm{CH}), 139.1$ (C), $138.6(\mathrm{CH}), 134.5(\mathrm{C}), 134.3(\mathrm{C}), 129.1(\mathrm{CH}), 128.5(\mathrm{CH}), 123.1(\mathrm{CH}), 40.9(\mathrm{CH}), 36.2$ $\left(\mathrm{CH}_{2}\right), 18.8\left(\mathrm{CH}_{3}\right) ; \mathrm{m} / \mathrm{z}\left(\mathrm{ES}^{+}\right) 331[\mathrm{MNa}]^{+}$; HRMS $\left(\mathrm{ES}^{+}\right)$calcd. for $\mathrm{C}_{14} \mathrm{H}_{13} \mathrm{CIN}_{2} \mathrm{NaO}_{2} \mathrm{~S}[\mathrm{MNa}]^{+}$: 331.0278, found: 331.0281 . Data consistent with published values. ${ }^{6}$ 


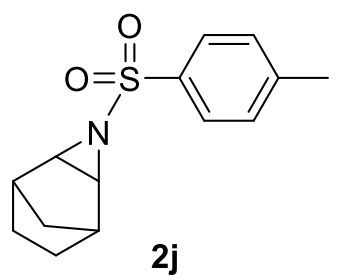

3-Tosyl-3-azatricyclo[3.2.1.0 $0^{2,4}$ octane 2j. Using 1a (44 mg, 0.102 $\mathrm{mmol})$, norbornene $(95 \mathrm{mg}, 1.01 \mathrm{mmol})$ and $(\mathrm{pyr})_{4} \mathrm{Cu}(\mathrm{OTf})_{2}(7 \mathrm{mg}$, $0.010 \mathrm{mmol}), \mathbf{2 j}$ (19 $\mathrm{mg}, 0.072 \mathrm{mmol}, 71 \%$ ) was produced as a clear oil. IR (film): 2968 (C-H), $2874(\mathrm{C}-\mathrm{H}), 1599(\mathrm{C}-\mathrm{H}), 1351$ (S=O), 1158 $(\mathrm{S}=\mathrm{O}) \mathrm{cm}^{-1} ; \delta_{\mathrm{H}}\left(500 \mathrm{MHz}, \mathrm{CDCl}_{3}\right): 7.81(2 \mathrm{H}, \mathrm{d}, J=8.1 \mathrm{~Hz}, \operatorname{ArH}), 7.32(2 \mathrm{H}, \mathrm{d}, J=8.1 \mathrm{~Hz}$, $\mathrm{ArH}), 2.92-2.91(2 \mathrm{H}, \mathrm{m}, \mathrm{NCH}), 2.45-2.43\left(5 \mathrm{H}, \mathrm{m}, \mathrm{ArCH}_{3}\right.$ and RR'R" $\left.\mathrm{CH}\right), 1.51-1.43(3 \mathrm{H}$, $\left.\mathrm{m}, \mathrm{R}_{2} \mathrm{CH}, \mathrm{CH}_{2}\right), 1.27-1.22\left(2 \mathrm{H}, \mathrm{m}, \mathrm{CH}_{2}\right), 0.77-0.73\left(1 \mathrm{H}, \mathrm{m}, \mathrm{R}_{2} \mathrm{CHH}\right) ; \delta_{\mathrm{c}}(125 \mathrm{MHz}$, $\left.\mathrm{CDCl}_{3}\right): 144.1(\mathrm{C}), 135.9(\mathrm{C}), 129.6(\mathrm{CH}), 127.7(\mathrm{CH}), 42.0(\mathrm{CH}), 35.8(\mathrm{CH}), 28.3\left(\mathrm{CH}_{2}\right), 25.6$ $\left(\mathrm{CH}_{2}\right), 21.6\left(\mathrm{CH}_{3}\right) ; \mathrm{m} / \mathrm{z}\left(\mathrm{ES}^{+}\right) 286[\mathrm{MNa}]^{+} ; \mathrm{HRMS}\left(\mathrm{ES}^{+}\right)$calcd. for $\mathrm{C}_{14} \mathrm{H}_{17} \mathrm{NNaO}_{2} \mathrm{~S}[\mathrm{MNa}]^{+}$: 286.0872, found: 286.0873. Data consistent with published values. ${ }^{1}$

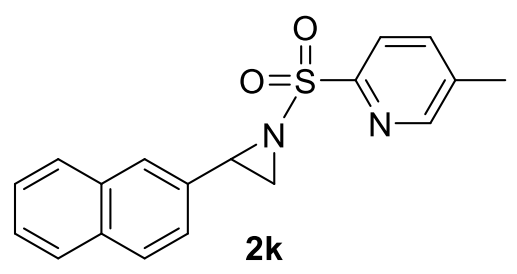

5-Methyl-2-[[2-(naphthalen-2-yl)aziridin-1-yl]sulfonyl] pyridine

2k. Using 1d (44 mg, $0.102 \mathrm{mmol})$, 2-vinylnaphthalene (155 mg, $1.01 \mathrm{mmol})$ and $(\mathrm{pyr})_{4} \mathrm{Cu}(\mathrm{OTf})_{2}(7 \mathrm{mg}, 0.010 \mathrm{mmol}), 2 \mathbf{k}(24 \mathrm{mg}$, $0.074 \mathrm{mmol}, 73 \%$ ) was produced as a clear oil. IR (film): 3051 $(\mathrm{C}-\mathrm{H}), 1572(\mathrm{C}=\mathrm{C}), 1325(\mathrm{~S}=\mathrm{O}), 1170(\mathrm{~S}=\mathrm{O}) \mathrm{cm}^{-1} ; \delta_{\mathrm{H}}\left(500 \mathrm{MHz}, \mathrm{CDCl}_{3}\right): 8.58-8.53(1 \mathrm{H}$, m, ArH), $8.04(1 \mathrm{H}, \mathrm{d}, J=8.0 \mathrm{~Hz}, \operatorname{Ar} H), 7.81-7.76(4 \mathrm{H}, \mathrm{m}, \operatorname{ArH}), 7.73-7.69(1 \mathrm{H}, \mathrm{m}, \operatorname{Ar} H)$, $7.49-7.44(2 \mathrm{H}, \mathrm{m}, \mathrm{ArH}), 7.34-7.30(1 \mathrm{H}, \mathrm{m}, \operatorname{ArH}), 4.15(1 \mathrm{H}, \mathrm{dd}, J=4.7,7.2 \mathrm{~Hz}, \operatorname{ArCH})$, $3.28(1 \mathrm{H}, \mathrm{d}, J=7.2 \mathrm{~Hz}, \mathrm{CH} H), 2.61(1 \mathrm{H}, \mathrm{d}, J=4.7 \mathrm{~Hz}, \mathrm{CHH}), 2.42\left(3 \mathrm{H}, \mathrm{s}, \operatorname{ArCH}_{3}\right) ; \delta_{\mathrm{C}}(125$ $\left.\mathrm{MHz}, \mathrm{CDCl}_{3}\right): 153.9(\mathrm{C}), 150.9(\mathrm{CH}), 138.3(\mathrm{C}), 138.2(\mathrm{CH}), 133.2(\mathrm{C}), 133.1(\mathrm{C}), 132.4(\mathrm{C})$, $128.4(\mathrm{CH}), 127.8(\mathrm{CH}), 127.7(\mathrm{CH}), 126.4(\mathrm{CH}), 126.35(\mathrm{CH}), 126.32(\mathrm{CH}), 123.9(\mathrm{CH})$, $122.8(\mathrm{CH}), 41.7(\mathrm{CH}), 36.1\left(\mathrm{CH}_{2}\right), 18.6\left(\mathrm{CH}_{3}\right) ; \mathrm{m} / \mathrm{z}\left(\mathrm{ES}^{+}\right) 347[\mathrm{MNa}]^{+}$; HRMS (ES $)$calcd. for $\mathrm{C}_{18} \mathrm{H}_{16} \mathrm{~N}_{2} \mathrm{NaO}_{2} \mathrm{~S}$ [MNa] ${ }^{+}: 347.0825$, found: 347.0822 . Data consistent with published values. ${ }^{4}$ 


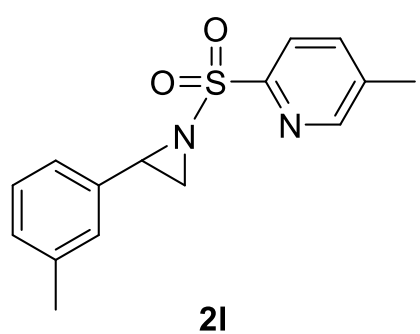

2I

5-Methyl-2-[[2-(m-tolyl)aziridin-1-yl]sulfonyl]pyridine 2l. Using 1d (44 mg, $0.102 \mathrm{mmol})$, 3-methylstyrene (119 mg, $1.01 \mathrm{mmol})$ and $(\mathrm{pyr})_{4} \mathrm{Cu}(\mathrm{OTf})_{2}(7 \mathrm{mg}, 0.010 \mathrm{mmol}), 2 \mathrm{l}(24 \mathrm{mg}, 0.083 \mathrm{mmol}$, $82 \%)$ was produced as a clear oil. IR (film): $1655(\mathrm{C}=\mathrm{C}), 1327$ $(\mathrm{S}=0), 1172(\mathrm{~S}=\mathrm{O}) \mathrm{cm}^{-1} ; \delta_{\mathrm{H}}\left(500 \mathrm{MHz}, \mathrm{CDCl}_{3}\right): 8.57-8.55(1 \mathrm{H}$, m, ArH), $8.02(1 \mathrm{H}, \mathrm{d}, J=8.0 \mathrm{~Hz}, \operatorname{Ar} H), 7.74-7.70(1 \mathrm{H}, \mathrm{m}, \operatorname{ArH}), 7.20-7.16(1 \mathrm{H}, \mathrm{m}, \operatorname{Ar} H)$, $7.10-7.05(3 \mathrm{H}, \mathrm{m}, \operatorname{ArH}), 3.96(1 \mathrm{H}, \mathrm{dd}, J=4.7,7.2 \mathrm{~Hz}, \operatorname{ArCH}), 3.18(1 \mathrm{H}, \mathrm{d}, J=7.2 \mathrm{~Hz}$, $\mathrm{CHH}), 2.50(1 \mathrm{H}, \mathrm{d}, J=4.7 \mathrm{~Hz}, \mathrm{CHH}), 2.50\left(3 \mathrm{H}, \mathrm{s}, \mathrm{ArCH}_{3}\right), 2.30\left(3 \mathrm{H}, \mathrm{s}, \mathrm{ArCH}_{3}\right) ; \delta_{\mathrm{C}}(125 \mathrm{MHz}$, $\left.\mathrm{CDCl}_{3}\right): 153.3(\mathrm{C}), 150.8(\mathrm{CH}), 138.3(\mathrm{C}), 138.2(\mathrm{C}), 138.1(\mathrm{CH}), 134.9(\mathrm{C}), 129.1(\mathrm{CH})$, $128.4(\mathrm{CH}), 127.3(\mathrm{CH}), 123.9(\mathrm{CH}), 122.8(\mathrm{CH}), 41.3(\mathrm{CH}), 36.1\left(\mathrm{CH}_{2}\right), 21.3\left(\mathrm{CH}_{3}\right), 18.6$ $\left(\mathrm{CH}_{3}\right) ; \mathrm{m} / \mathrm{z}\left(\mathrm{ES}^{+}\right) 311[\mathrm{MNa}]^{+} ; \mathrm{HRMS}\left(\mathrm{ES}^{+}\right)$calcd. for $\mathrm{C}_{15} \mathrm{H}_{16} \mathrm{~N}_{2} \mathrm{NaO}_{2} \mathrm{~S}[\mathrm{MNa}]^{+}:$311.0825, found: 311.0824 .

\section{Alternative flow synthesis of $2 d$ and $2 f$ in a tube reactor}

An inexpensive tube reactor was assembled by connecting computer controlled Tricontinent C3000 syringe pumps to a custom built flow reactor constructed out of $1 / 16$ " ID PTFE tubing and a $\mathrm{T}$ connector. 1a $(0.10 \mathrm{mmol}, 1.0 \mathrm{eq})$ was dissolved in $\mathrm{CH}_{2} \mathrm{Cl}_{2}(2.0 \mathrm{ml})$ to give solution $\mathrm{A}$. Alkene $(1.00 \mathrm{mmol}, 10 \mathrm{eq})$

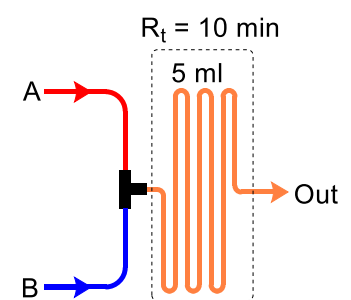

Figure S2. Tube reactor used for alternative synthesis of $\mathbf{2} \mathbf{d}$ and $\mathbf{2} \mathbf{f}$. and $(\mathrm{pyr})_{4} \mathrm{Cu}(\mathrm{OTf})_{2}(0.01 \mathrm{mmol}, 0.1 \mathrm{eq})$ were dissolved in anhydrous $\mathrm{MeCN}(2.0 \mathrm{ml})$ to give solution B. Solutions A and B were combined in the tube reactor at room temperature, with a residence time of $10 \mathrm{~min}$. The outlet stream was quenched thermally at $-78{ }^{\circ} \mathrm{C}$. Upon completion of the run, additional quantities of $\mathrm{CH}_{2} \mathrm{Cl}_{2}$ and $\mathrm{MeCN}$ were passed through the tube reactor at $A$ and $B$ respectively to ensure all the product was collected. The resulting mixture was concentrated under vacuum and purified by gradient column chromatography (5:1 ${ }^{n}$ hexane:EtOAc, then $3: 1^{n}$ hexane:EtOAc) to give the following products: 
<smiles>Cc1ccc(S(=O)(=O)N2CC2c2cccc(C)c2)cc1</smiles>

2d

2-(m-Tolyl)-1-tosylaziridine 2d. Using 1a (44 mg, $0.102 \mathrm{mmol})$, 3-methylstyrene $(119 \mathrm{mg}, 1.01 \mathrm{mmol}, 10 \mathrm{eq})$ and $(\mathrm{pyr})_{4} \mathrm{Cu}(\mathrm{OTf})_{2}$ (7 mg, $0.010 \mathrm{mmol}), 2 \mathrm{~d}(20 \mathrm{mg}, 0.070 \mathrm{mmol}, 68 \%$ ) was produced as a clear oil. Data as that reported above.

2-(Naphthalen-2-yl)-1-tosylaziridine 2f. Using 1a (44 mg,<smiles>CCCC1CN1S(=O)(=O)c1ccc(C)cc1</smiles>

$0.102 \mathrm{mmol}), 2$-vinylnaphthalene $(155 \mathrm{mg}, 1.01 \mathrm{mmol})$ and $(\text { pyr })_{4} \mathrm{Cu}(\mathrm{OTf})_{2}(7 \mathrm{mg}, 0.010 \mathrm{mmol}), 2 \mathrm{f}(24 \mathrm{mg}, 0.074 \mathrm{mmol}$, $73 \%$ ) was produced as a clear oil. Data as that reported above.

\section{Flow synthesis of amines 3a-r by telescoped aziridination/ring-opening}

A custom flow system was assembled by connecting computer controlled Tricontinent C3000 syringe pumps to a custom built tube reactor constructed out of $1 / 16$ " ID PTFE tubing and T connectors. The tube reactor was maintained at room temperature. $A r l=\mathrm{NSO}_{2} \mathrm{R} \quad(0.10$ mmol, 1.0 eq) was dissolved in $\mathrm{CH}_{2} \mathrm{Cl}_{2}(2.0 \mathrm{ml})$ to give solution A. Alkene $(0.50 \mathrm{mmol}, 5.0 \mathrm{eq})$ and

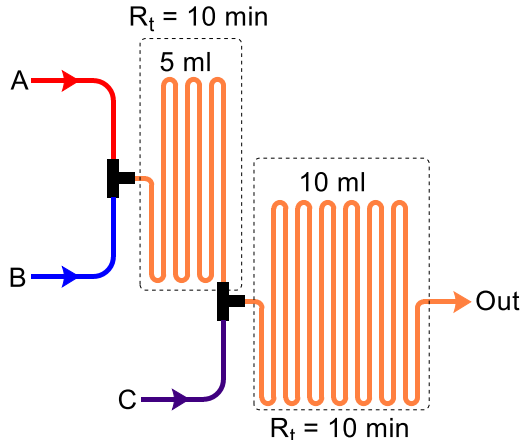

Figure S3. Tube reactor used for aziridination of alkenes and subsequent ring opening. $(\mathrm{pyr})_{4} \mathrm{Cu}(\mathrm{OTf})_{2}(0.01 \mathrm{mmol}, 0.1 \mathrm{eq})$ were dissolved in anhydrous $\mathrm{MeCN}(2.0 \mathrm{ml})$ to give solution B. Solution C was made by dissolving the nucleophile and acid in a suitable solvent $(4.0 \mathrm{ml})$; details are given for each entry. Solutions $A$ and $B$ were combined in the first section of the tube reactor at room temperature, with a residence time of $10 \mathrm{~min}$. Solution $\mathrm{C}$ was then added in the second section of the tube reactor at room temperature, with a residence time of $10 \mathrm{~min}$. The outlet stream was quenched into a solution of $\mathrm{NEt}_{3}(0.50$ $\mathrm{mmol})$ in $\mathrm{CHCl}_{3}(2 \mathrm{ml})$ for alcohol ring openings, or saturated aq $\mathrm{NaHCO}_{3}(5 \mathrm{ml})$ for $\mathrm{HCl}$ ring 
openings. Upon completion of the run, additional quantities of $\mathrm{CH}_{2} \mathrm{Cl}_{2}$ were passed through the tube reactor to ensure all the product was collected. The resulting mixture was concentrated in vacuo and purified by gradient column chromatography ( $3: 1^{n}$ hexane:EtOAc, then $1: 1^{n}$ hexane:EtOAc) to give the following products:<smiles>COC(CNS(=O)(=O)c1ccccc1[N+](=O)[O-])c1ccccc1</smiles>

\section{N-(2-Methoxy-2-phenylethyl)-2-nitrobenzenesulfonamide $3 a$.}

Using 1c (47 mg, $0.102 \mathrm{mmol})$ to make solution A; styrene (53 $\mathrm{mg}, 0.509 \mathrm{mmol})$ and $(\mathrm{pyr})_{4} \mathrm{Cu}(\mathrm{OTf})_{2}(7 \mathrm{mg}, 0.010 \mathrm{mmol})$ to make solution $\mathrm{B} ; \mathrm{H}_{2} \mathrm{SO}_{4}(15 \mathrm{mg}, 0.153 \mathrm{mmol})$ in $\mathrm{MeOH}(4.0 \mathrm{ml})$ to make solution $\mathrm{C}, 3 \mathrm{a}(25 \mathrm{mg}$, $0.074 \mathrm{mmol}, 73 \%)$ was obtained as a clear oil. IR (film): $3342(\mathrm{~N}-\mathrm{H}), 2932(\mathrm{C}-\mathrm{H}), 1575$ $(\mathrm{C}=\mathrm{C}), 1343(\mathrm{~S}=\mathrm{O}), 1167(\mathrm{~S}=\mathrm{O}) \mathrm{cm}^{-1} ; \delta_{\mathrm{H}}\left(500 \mathrm{MHz}, \mathrm{CDCl}_{3}\right): 8.11-8.06(1 \mathrm{H}, \mathrm{m}, \mathrm{ArH}), 7.91$ - $7.87(1 \mathrm{H}, \mathrm{m}, \mathrm{ArH}), 7.75-7.69(2 \mathrm{H}, \mathrm{m}, \mathrm{ArH}), 7.35-7.27(3 \mathrm{H}, \mathrm{m}, \operatorname{ArH}), 7.23-7.20(2 \mathrm{H}, \mathrm{m}$, $\operatorname{ArH}), 5.88(1 \mathrm{H}, \mathrm{dd}, J=3.0,8.1 \mathrm{~Hz}, \mathrm{NH}), 4.25(1 \mathrm{H}, \mathrm{dd}, J=3.6,9.2 \mathrm{~Hz}, \operatorname{ArCH}), 3.43-3.37$ $(1 \mathrm{H}, \mathrm{m}, \mathrm{CH} H), 3.16\left(3 \mathrm{H}, \mathrm{s}, \mathrm{OCH}_{3}\right), 3.15-3.10(1 \mathrm{H}, \mathrm{m}, \mathrm{CHH}) ; \delta_{\mathrm{C}}\left(125 \mathrm{MHz}, \mathrm{CDCl}_{3}\right): 148.0$ (C), $138.0(\mathrm{C}), 134.0(\mathrm{C}), 133.5(\mathrm{CH}), 132.8(\mathrm{CH}), 131.0(\mathrm{CH}), 128.7(\mathrm{CH}), 128.5(\mathrm{CH}), 126.6$ $(\mathrm{CH}), 125.4(\mathrm{CH}), 82.0(\mathrm{CH}), 56.8\left(\mathrm{CH}_{3}\right), 50.0\left(\mathrm{CH}_{2}\right) ; \mathrm{m} / \mathrm{z}\left(\mathrm{ES}^{+}\right) 359$ [MNa] ${ }^{+}$; HRMS $\left(\mathrm{ES}^{+}\right)$ calcd. for $\mathrm{C}_{15} \mathrm{H}_{16} \mathrm{~N}_{2} \mathrm{NaO}_{5} \mathrm{~S}$ [MNa] ${ }^{+}: 359.0672$, found: 359.0674 .

\section{$\mathrm{N}$-[2-(Benzyloxy)-2-phenylethyl]-2-nitrobenzenesulfonamide}<smiles>O=[N+]([O-])c1ccccc1S(=O)(=O)NCC(OCc1ccccc1)c1ccccc1</smiles>
a clear oil. IR (film): $3337(\mathrm{~N}-\mathrm{H}), 1593(\mathrm{C}=\mathrm{C}), 1345(\mathrm{~S}=\mathrm{O}), 1169$

$(\mathrm{S}=\mathrm{O}) \mathrm{cm}^{-1} ; \delta_{\mathrm{H}}\left(500 \mathrm{MHz}, \mathrm{CDCl}_{3}\right): 8.09-8.05(1 \mathrm{H}, \mathrm{m}, \operatorname{ArH}), 7.87-7.82(1 \mathrm{H}, \mathrm{m}, \operatorname{ArH}), 7.73$ - $7.66(2 \mathrm{H}, \mathrm{m}, \mathrm{ArH}), 7.40-7.25(10 \mathrm{H}, \mathrm{m}, \mathrm{ArH}), 5.92(1 \mathrm{H}, \mathrm{dd}, J=3.1,8.3 \mathrm{~Hz}, \mathrm{NH}), 4.51(1 \mathrm{H}$, $\mathrm{dd}, J=3.6,9.1 \mathrm{~Hz},(\mathrm{BnO}) \mathrm{CH}), 4.44(1 \mathrm{H}, \mathrm{d}, J=11.4 \mathrm{~Hz}, \mathrm{OCH} H \mathrm{Ph}), 4.23(1 \mathrm{H}, \mathrm{d}, J=11.4 \mathrm{~Hz}$, OCHHPh), $3.50-3.44(1 \mathrm{H}, \mathrm{m}, \mathrm{CH}), 3.27-3.21(1 \mathrm{H}, \mathrm{m}, \mathrm{CHH}) ; \delta_{\mathrm{c}}\left(125 \mathrm{MHz}, \mathrm{CDCl}_{3}\right)$ : 
$148.0(\mathrm{C}), 138.1(\mathrm{C}), 137.3(\mathrm{C}), 133.9(\mathrm{C}), 133.4(\mathrm{CH}), 132.7(\mathrm{CH}), 130.9(\mathrm{CH}), 128.8(\mathrm{CH})$, $128.7(\mathrm{CH}), 128.5(\mathrm{CH}), 127.96(\mathrm{CH}), 127.95(\mathrm{CH}), 126.7(\mathrm{CH}), 125.5(\mathrm{CH}), 79.6(\mathrm{CH}), 70.8$ $\left(\mathrm{CH}_{2}\right), 50.0\left(\mathrm{CH}_{2}\right) ; \mathrm{m} / \mathrm{z}\left(\mathrm{ES}^{+}\right) 435[\mathrm{MNa}]^{+}$; HRMS $\left(\mathrm{ES}^{+}\right)$calcd. for $\mathrm{C}_{21} \mathrm{H}_{20} \mathrm{~N}_{2} \mathrm{NaO}_{5} \mathrm{~S}[\mathrm{MNa}]^{+}$: 435.0985, found: 435.0987 .<smiles>O=[N+]([O-])c1ccccc1S(=O)(=O)NCC(Cl)c1ccccc1</smiles>

\section{N-2-Chloro-2-phenylethyl)-2-nitrobenzenesulfonamide 3c.}

Using 1c (47 mg, $0.102 \mathrm{mmol})$ to make solution A; styrene (53 $\mathrm{mg}, 0.509 \mathrm{mmol})$ and $(\mathrm{pyr})_{4} \mathrm{Cu}(\mathrm{OTf})_{2}(7 \mathrm{mg}, 0.010 \mathrm{mmol})$ to make

solution $\mathrm{B}$; $\mathrm{HCl}\left(2 \mathrm{M}\right.$ in $\left.\mathrm{Et}_{2} \mathrm{O}, 1.0 \mathrm{ml}, 2.0 \mathrm{mmol}\right)$ in $\mathrm{CH}_{2} \mathrm{Cl}_{2}(3.0 \mathrm{ml})$ to make solution $\mathrm{C}, 3 \mathrm{c}$ (19 $\mathrm{mg}, 0.056 \mathrm{mmol}, 55 \%)$ was obtained as a clear oil. IR (film): $3346(\mathrm{~N}-\mathrm{H}), 1594(\mathrm{C}=\mathrm{C}), 1348$ $(\mathrm{S}=\mathrm{O}), 1166(\mathrm{~S}=\mathrm{O}) \mathrm{cm}^{-1} ; \delta_{\mathrm{H}}\left(500 \mathrm{MHz}, \mathrm{CDCl}_{3}\right): 8.14-8.09(1 \mathrm{H}, \mathrm{m}, \mathrm{ArH}), 7.92-7.88(1 \mathrm{H}$, m, ArH), $7.78-7.73(1 \mathrm{H}, \mathrm{m}, \mathrm{ArH}), 7.37-7.29(5 \mathrm{H}, \mathrm{m}, \mathrm{ArH}), 5.83-5.70(1 \mathrm{H}, \mathrm{m}, \mathrm{NH}), 4.95$ $(1 \mathrm{H}, \mathrm{dd}, J=5.8,8.1 \mathrm{~Hz}, \mathrm{ArCH}), 3.72-3.66(1 \mathrm{H}, \mathrm{m}, \mathrm{CH} H), 3.63-3.57(1 \mathrm{H}, \mathrm{m}, \mathrm{CHH}) ; \delta_{\mathrm{C}}$ (125 MHz, $\left.\mathrm{CDCl}_{3}\right)$ : $147.9(\mathrm{C}), 137.5(\mathrm{C}), 134.0(\mathrm{C}), 133.8(\mathrm{CH}), 133.0(\mathrm{CH}), 130.7(\mathrm{CH})$, $129.2(\mathrm{CH}), 129.0(\mathrm{CH}), 127.1(\mathrm{CH}), 125.6(\mathrm{CH}), 61.4(\mathrm{CH}), 51.0\left(\mathrm{CH}_{2}\right) ; \mathrm{m} / \mathrm{z}\left(\mathrm{ES}^{+}\right) 363$ [MNa] ${ }^{+}$; HRMS $\left(\mathrm{ES}^{+}\right)$calcd. for $\mathrm{C}_{14} \mathrm{H}_{13} \mathrm{CIN}_{2} \mathrm{NaO}_{4} \mathrm{~S}$ [MNa] ${ }^{+}: 363.0177$, found: 363.0183.

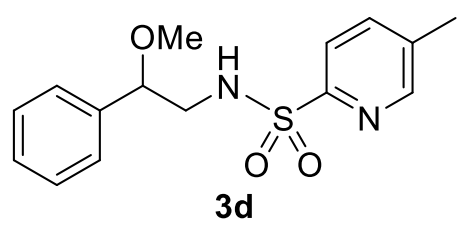

\section{N-(2-Methoxy-2-phenylethyl)-5-methylpyridine-2-sulfonamide}

3d. Using 1d (44 mg, mmol, $0.102 \mathrm{mmol}$ ) to make solution $A$; styrene $(53 \mathrm{mg}, 0.509 \mathrm{mmol})$ and $(\mathrm{pyr})_{4} \mathrm{Cu}(\mathrm{OTf})_{2}(7 \mathrm{mg}, 0.010$ mmol) to make solution $\mathrm{B} ; \mathrm{H}_{2} \mathrm{SO}_{4}(15 \mathrm{mg}, 0.153 \mathrm{mmol})$ in $\mathrm{MeOH}(4.0 \mathrm{ml})$ to make solution $\mathrm{C}$, 3d (20 mg, $0.065 \mathrm{mmol}, 64 \%$ ) was obtained as a clear oil. IR (film): $3245(\mathrm{~N}-\mathrm{H}), 1572$ $(\mathrm{C}=\mathrm{C}), 1332(\mathrm{~S}=\mathrm{O}), 1171(\mathrm{~S}=\mathrm{O}) \mathrm{cm}^{-1} ; \delta_{\mathrm{H}}\left(500 \mathrm{MHz}, \mathrm{CDCl}_{3}\right): 8.56-8.50(1 \mathrm{H}, \mathrm{m}, \mathrm{ArH}), 7.88$ - $7.85(1 \mathrm{H}, \mathrm{m}, \mathrm{ArH}), 7.69-7.65(1 \mathrm{H}, \mathrm{m}, \mathrm{ArH}), 7.35-7.27(3 \mathrm{H}, \mathrm{m}, \mathrm{ArH}), 7.25-7.21(2 \mathrm{H}, \mathrm{m}$, $\operatorname{ArH}), 5.34(1 \mathrm{H}, \mathrm{dd}, J=2.8,9.2 \mathrm{~Hz}, \mathrm{NH}), 4.25(1 \mathrm{H}, \mathrm{dd}, J=3.7,9.3 \mathrm{~Hz}, \operatorname{ArCH}), 3.25-3.29$ $(1 \mathrm{H}, \mathrm{m}, \mathrm{CH} H), 3.20\left(3 \mathrm{H}, \mathrm{s}, \mathrm{OCH}_{3}\right), 3.13-3.06(1 \mathrm{H}, \mathrm{m}, \mathrm{CHH}), 2.43\left(3 \mathrm{H}, \mathrm{s}, \mathrm{ArCH}_{3}\right) ; \delta_{\mathrm{C}}(125$ $\left.\mathrm{MHz}, \mathrm{CDCl}_{3}\right): 154.9(\mathrm{C}), 150.6(\mathrm{CH}), 138.3(\mathrm{C}), 138.0(\mathrm{CH}), 137.2(\mathrm{C}), 128.7(\mathrm{CH}), 128.4$ 
$(\mathrm{CH}), 126.7(\mathrm{CH}), 121.7(\mathrm{CH}), 82.4(\mathrm{CH}), 56.8\left(\mathrm{CH}_{3}\right), 49.8\left(\mathrm{CH}_{2}\right), 18.5\left(\mathrm{CH}_{3}\right) ; \mathrm{m} / \mathrm{z}\left(\mathrm{ES}^{+}\right) 329$ [MNa] ${ }^{+}$; HRMS $\left(\mathrm{ES}^{+}\right)$calcd. for $\mathrm{C}_{15} \mathrm{H}_{18} \mathrm{~N}_{2} \mathrm{NaO}_{3} \mathrm{~S}$ [MNa] $]^{+}: 329.0930$, found: 329.0927.<smiles>COC(C)(CNS(=O)(=O)c1ccccc1[N+](=O)[O-])c1ccccc1</smiles>

\section{N-(2-Methoxy-2-phenylpropyl)-2-nitrobenzenesulfonamide 3e.} Using 1c (47 mg, $0.102 \mathrm{mmol})$ to make solution $\mathrm{A}$; $\mathrm{a}-$ methylstyrene $(60 \mathrm{mg}, 0.508 \mathrm{mmol})$ and $(\mathrm{pyr})_{4} \mathrm{Cu}(\mathrm{OTf})_{2}(7 \mathrm{mg}$, $0.010 \mathrm{mmol})$ to make solution $\mathrm{B} ; \mathrm{H}_{2} \mathrm{SO}_{4}(15 \mathrm{mg}, 0.153 \mathrm{mmol})$ in $\mathrm{MeOH}(4.0 \mathrm{ml})$ to make solution C, 3 e (24 mg, $0.068 \mathrm{mmol}, 67 \%)$ was obtained as a clear oil. IR (film): $3346(\mathrm{~N}-\mathrm{H})$, $1593(\mathrm{C}=\mathrm{C}), 1355(\mathrm{~S}=\mathrm{O}), 1168(\mathrm{~S}=\mathrm{O}) \mathrm{cm}^{-1} ; \delta_{\mathrm{H}}\left(500 \mathrm{MHz}, \mathrm{CD}_{2} \mathrm{Cl}_{2}\right): 8.02-7.98(1 \mathrm{H}, \mathrm{m}, \mathrm{ArH})$, $7.88-7.84(1 \mathrm{H}, \mathrm{m}, \mathrm{ArH}), 7.77-7.70(2 \mathrm{H}, \mathrm{m}, \operatorname{ArH}), 7.35-7.32(4 \mathrm{H}, \mathrm{m}, \operatorname{ArH}), 7.30-7.25$ $(1 \mathrm{H}, \mathrm{m}, \mathrm{ArH}), 5.70-5.60(1 \mathrm{H}, \mathrm{m}, \mathrm{NH}), 3.35-3.29(1 \mathrm{H}, \mathrm{m}, \mathrm{CH}), 3.25-3.21(1 \mathrm{H}, \mathrm{m}, \mathrm{CHH})$, $3.07\left(3 \mathrm{H}, \mathrm{s}, \mathrm{OCH}_{3}\right), 1.63\left(3 \mathrm{H}, \mathrm{s}, \mathrm{CH}_{3}\right) ; \delta_{\mathrm{C}}\left(125 \mathrm{MHz}, \mathrm{CD}_{2} \mathrm{Cl}_{2}\right): 147.8(\mathrm{C}), 141.7(\mathrm{C}), 133.55$ (C), $133.46(\mathrm{CH}), 132.8(\mathrm{CH}), 130.9(\mathrm{CH}), 128.4(\mathrm{CH}), 127.7(\mathrm{CH}), 126.1(\mathrm{CH}), 125.2(\mathrm{CH})$, 78.1 (C), $54.4\left(\mathrm{CH}_{2}\right), 50.3\left(\mathrm{CH}_{3}\right), 20.3\left(\mathrm{CH}_{3}\right) ; \mathrm{m} / \mathrm{z}\left(\mathrm{ES}^{+}\right) 373[\mathrm{MNa}]^{+}$; HRMS (ES $\left.{ }^{+}\right)$calcd. for $\mathrm{C}_{16} \mathrm{H}_{18} \mathrm{~N}_{2} \mathrm{NaO}_{5} \mathrm{~S}[\mathrm{MNa}]^{+}: 373.0829$, found: 373.0831.<smiles>CCOC(C)(CNS(=O)(=O)c1ccccc1[N+](=O)[O-])c1ccccc1</smiles>

\section{$\mathrm{N}$-(2-Ethoxy-2-phenylpropyl)-2-nitrobenzenesulfonamide $3 f$.} Using 1c (47 mg, $0.102 \mathrm{mmol}$ ) to make solution $\mathrm{A}$; $\mathrm{a}-$ methylstyrene $(60 \mathrm{mg}, 0.508 \mathrm{mmol})$ and $(\mathrm{pyr})_{4} \mathrm{Cu}(\mathrm{OTf})_{2}(7 \mathrm{mg}$, $0.010 \mathrm{mmol})$ to make solution $\mathrm{B} ; \mathrm{H}_{2} \mathrm{SO}_{4}(15 \mathrm{mg}, 0.153 \mathrm{mmol})$ in $\mathrm{EtOH}(4.0 \mathrm{ml})$ to make solution C, $3 f$ (21 mg, $0.058 \mathrm{mmol}, 56 \%)$ was obtained as a clear oil. IR (film): $3350(\mathrm{~N}-\mathrm{H})$, $1593(\mathrm{C}=\mathrm{C}), 1352(\mathrm{~S}=\mathrm{O}), 1169(\mathrm{~S}=\mathrm{O}) \mathrm{cm}^{-1} ; \delta_{\mathrm{H}}\left(500 \mathrm{MHz}, \mathrm{CD}_{3} \mathrm{CN}\right): 7.99-7.95(1 \mathrm{H}, \mathrm{m}$, $\operatorname{ArH}), 7.89-7.85(1 \mathrm{H}, \mathrm{m}, \operatorname{ArH}), 7.83-7.76(2 \mathrm{H}, \mathrm{m}, \operatorname{ArH}), 7.39-7.30(4 \mathrm{H}, \mathrm{m}, \operatorname{ArH}), 7.29-$ $7.24(1 \mathrm{H}, \mathrm{m}, \mathrm{ArH}), 5.94-5.71(1 \mathrm{H}, \mathrm{m}, \mathrm{NH}), 3.26-3.21\left(3 \mathrm{H}, \mathrm{m}, \mathrm{CH}_{2}\right.$ and $\left.\mathrm{OCH} H\right), 3.16-$ $3.09(1 \mathrm{H}, \mathrm{m}, \mathrm{OCHH}), 1.11\left(3 \mathrm{H}, \mathrm{t}, J=6.9 \mathrm{~Hz}, \mathrm{OCH}_{2} \mathrm{CH}_{3}\right) ; \delta_{\mathrm{C}}\left(125 \mathrm{MHz}, \mathrm{CD}_{3} \mathrm{CN}\right): 148.3(\mathrm{C})$, $143.1(\mathrm{C}), 134.4(\mathrm{CH}), 133.4(\mathrm{CH}), 133.2(\mathrm{C}), 130.9(\mathrm{CH}), 128.7(\mathrm{CH}), 127.9(\mathrm{CH}), 126.5$ 
$(\mathrm{CH}), 125.5(\mathrm{CH}), 78.0(\mathrm{C}), 58.2\left(\mathrm{CH}_{2}\right), 54.1\left(\mathrm{CH}_{2}\right), 21.4\left(\mathrm{CH}_{3}\right), 15.2\left(\mathrm{CH}_{3}\right) ; \mathrm{m} / \mathrm{z}\left(\mathrm{ES}^{+}\right) 387$ [MNa] ${ }^{+}$; HRMS $\left(\mathrm{ES}^{+}\right)$calcd. for $\mathrm{C}_{17} \mathrm{H}_{20} \mathrm{~N}_{2} \mathrm{NaO}_{5} \mathrm{~S}[\mathrm{MH}]^{+}: 387.0985$, found: 387.0980 .<smiles>COC(CNS(=O)(=O)c1ccccc1[N+](=O)[O-])c1ccc2ccccc2c1</smiles>

\section{$\mathrm{N}$-[2-Methoxy-2-(naphthalen-2-yl)ethyl]-2-nitrobenzene} sulfonamide $3 \mathrm{~g}$. Using $1 \mathrm{c}(47 \mathrm{mg}, 0.102 \mathrm{mmol})$ to make solution A; 2-vinylnaphthalene (78 $\mathrm{mg}, 0.506 \mathrm{mmol})$ and (pyr) $)_{4} \mathrm{Cu}(\mathrm{OTf})_{2}(7 \mathrm{mg}, 0.010 \mathrm{mmol})$ to make solution $\mathrm{B} ; \mathrm{H}_{2} \mathrm{SO}_{4}(15 \mathrm{mg}, 0.153 \mathrm{mmol})$ in $\mathrm{MeOH}$ $(4.0 \mathrm{ml})$ to make solution $\mathrm{C}, 3 \mathrm{~g}(28 \mathrm{mg}, 0.072 \mathrm{mmol}, 71 \%)$ was obtained as a clear oil. IR (film): $3351(\mathrm{~N}-\mathrm{H}), 1594(\mathrm{C}=\mathrm{C}), 1343(\mathrm{~S}=\mathrm{O}), 1167(\mathrm{~S}=\mathrm{O}) \mathrm{cm}^{-1} ; \delta_{\mathrm{H}}\left(500 \mathrm{MHz}, \mathrm{CDCl}_{3}\right): 8.01-$ $7.98(1 \mathrm{H}, \mathrm{m}, \operatorname{Ar} H), 7.81-7.74(4 \mathrm{H}, \mathrm{m}, \operatorname{ArH}), 7.67(1 \mathrm{H}, \mathrm{s}, \operatorname{Ar} H), 7.62-7.57(2 \mathrm{H}, \mathrm{m}, \operatorname{Ar} H)$, $7.50-7.46(2 \mathrm{H}, \mathrm{m}, \mathrm{ArH}), 7.33-7.29(1 \mathrm{H}, \mathrm{m}, \mathrm{ArH}), 5.91(1 \mathrm{H}, \mathrm{dd}, J=3.7,7.8 \mathrm{~Hz}, \mathrm{NH}), 4.43$ $(1 \mathrm{H}, \mathrm{dd}, J=3.8,8.5 \mathrm{~Hz}, \operatorname{ArCH}), 3.56-3.50(1 \mathrm{H}, \mathrm{m}, \mathrm{CH} H), 3.31-3.25(1 \mathrm{H}, \mathrm{m}, \mathrm{CHH}), 3.22$ $\left(3 \mathrm{H}, \mathrm{s}, \mathrm{OCH}_{3}\right) ; \delta_{\mathrm{C}}\left(125 \mathrm{MHz}, \mathrm{CDCl}_{3}\right): 147.8(\mathrm{C}), 135.4(\mathrm{C}), 134.0(\mathrm{C}), 133.4(\mathrm{C}), 133.2(\mathrm{CH})$, $133.1(\mathrm{C}), 132.7(\mathrm{CH}), 130.7(\mathrm{CH}), 128.7(\mathrm{CH}), 127.9(\mathrm{CH}), 127.8(\mathrm{CH}), 126.5(\mathrm{CH}), 126.3$ $(\mathrm{CH}), 126.0(\mathrm{CH}), 125.3(\mathrm{CH}), 123.9(\mathrm{CH}), 82.0(\mathrm{CH}), 57.0\left(\mathrm{CH}_{3}\right), 49.9\left(\mathrm{CH}_{2}\right) ; m / z\left(\mathrm{ES}^{+}\right) 409$ [MNa] ${ }^{+}$; HRMS $\left(\mathrm{ES}^{+}\right.$) calcd. for $\mathrm{C}_{19} \mathrm{H}_{18} \mathrm{~N}_{2} \mathrm{NaO}_{5} \mathrm{~S}[\mathrm{MNa}]^{+}: 409.0829$, found: 409.0827 .<smiles>Cc1ccc(S(=O)(=O)NCC(Cl)c2ccc3ccccc3c2)nc1</smiles>

$\mathrm{N}$-[2-Chloro-2-(naphthalen-2-yl)ethyl]-5-methylpyridine -2-sulfonamide $3 \mathrm{~h}$. Using 1d (44 $\mathrm{mg}, \mathrm{mmol}, 0.102 \mathrm{mmol})$ to make solution $\mathrm{A}$; 2-vinylnaphthalene $(78 \mathrm{mg}, 0.506$ $\mathrm{mmol})$ and $(\mathrm{pyr})_{4} \mathrm{Cu}(\mathrm{OTf})_{2}(7 \mathrm{mg}, 0.010 \mathrm{mmol})$ to make solution $\mathrm{B}$; $\mathrm{HCl}\left(2 \mathrm{M}\right.$ in $\mathrm{Et}_{2} \mathrm{O}, 1.0 \mathrm{ml}$, $2.0 \mathrm{mmol})$ in $\mathrm{CH}_{2} \mathrm{Cl}_{2}(3.0 \mathrm{ml})$ to make solution $\mathrm{C}, 3 \mathrm{~h}(18 \mathrm{mg}, 0.050 \mathrm{mmol}, 49 \%)$ was obtained as a crystalline solid. M.p. $131-132{ }^{\circ} \mathrm{C}$. IR (film): $3276(\mathrm{~N}-\mathrm{H}), 1601$ (C=C), 1333 $(\mathrm{S}=\mathrm{O}), 1170(\mathrm{~S}=\mathrm{O}) \mathrm{cm}^{-1} ; \delta_{\mathrm{H}}\left(500 \mathrm{MHz}, \mathrm{CDCl}_{3}\right): 8.43-8.38(1 \mathrm{H}, \mathrm{m}, \mathrm{ArH}), 7.86-7.83(1 \mathrm{H}$, m, ArH), $7.83-7.77(4 \mathrm{H}, \mathrm{m}, \mathrm{ArH}), 7.65-7.60(1 \mathrm{H}, \mathrm{m}, \operatorname{ArH}), 7.53-7.48(2 \mathrm{H}, \mathrm{m}, \operatorname{ArH}), 7.46$ - $7.42(1 \mathrm{H}, \mathrm{m}, \mathrm{ArH}), 5.53-5.45(1 \mathrm{H}, \mathrm{m}, \mathrm{NH}), 5.18(1 \mathrm{H}, \mathrm{dd}, J=6.5,7.8 \mathrm{~Hz}, \operatorname{ArCH}), 3.78-$ $3.71(1 \mathrm{H}, \mathrm{m}, \mathrm{CHH}), 3.70-3.63(1 \mathrm{H}, \mathrm{m}, \mathrm{CHH}), 2.37\left(3 \mathrm{H}, \mathrm{s}, \mathrm{ArCH}_{3}\right) ; \delta_{\mathrm{c}}\left(125 \mathrm{MHz}, \mathrm{CDCl}_{3}\right)$ : 
$154.7(\mathrm{C}), 150.5(\mathrm{CH}), 138.1(\mathrm{CH}), 137.4(\mathrm{C}), 135.1(\mathrm{C}), 133.4(\mathrm{C}), 132.9(\mathrm{C}), 128.9(\mathrm{CH})$, $128.1(\mathrm{CH}), 127.7(\mathrm{CH}), 127.0(\mathrm{CH}), 126.8(\mathrm{CH}), 126.7(\mathrm{CH}), 124.3(\mathrm{CH}), 121.7(\mathrm{CH}), 62.1$ $(\mathrm{CH}), \quad 50.7 \quad\left(\mathrm{CH}_{2}\right), \quad 18.5 \quad\left(\mathrm{CH}_{3}\right) ; \quad \mathrm{m} / \mathrm{z} \quad\left(\mathrm{ES}^{+}\right) \quad 383 \quad[\mathrm{MNa}]^{+} ; \quad \mathrm{HRMS} \quad\left(\mathrm{ES}^{+}\right)$calcd. for $\mathrm{C}_{18} \mathrm{H}_{17} \mathrm{CIN}_{2} \mathrm{NaO}_{2} \mathrm{~S}[\mathrm{MNa}]^{+}: 383.0591$, found: 383.0593.<smiles>COC(CNS(=O)(=O)c1ccccc1[N+](=O)[O-])c1cccc(C)c1</smiles>

N-[2-Methoxy-2-(m-tolyl)ethyl]-2-nitrobenzenesulfonamide $3 i$. Using 1c (47 mg, $0.102 \mathrm{mmol}$ ) to make solution $\mathrm{A}$; 3methylstyrene $(60 \mathrm{mg}, 0.508 \mathrm{mmol})$ and $(\mathrm{pyr})_{4} \mathrm{Cu}(\mathrm{OTf})_{2}(7 \mathrm{mg}$, $0.010 \mathrm{mmol})$ to make solution $\mathrm{B} ; \mathrm{H}_{2} \mathrm{SO}_{4}(15 \mathrm{mg}, 0.153 \mathrm{mmol})$ in $\mathrm{MeOH}(4.0 \mathrm{ml})$ to make solution C, $3 \mathbf{i}$ (27 mg, $0.077 \mathrm{mmol}, 76 \%)$ was obtained as a clear oil. IR (film): $3341(\mathrm{~N}-\mathrm{H})$, $1593(\mathrm{C}=\mathrm{C}), 1345(\mathrm{~S}=\mathrm{O}), 1167(\mathrm{~S}=\mathrm{O}) \mathrm{cm}^{-1} ; \delta_{\mathrm{H}}\left(500 \mathrm{MHz}, \mathrm{CDCl}_{3}\right): 8.11-8.07(1 \mathrm{H}, \mathrm{m}, \mathrm{ArH})$, $7.91-7.86(1 \mathrm{H}, \mathrm{m}, \operatorname{ArH}), 7.75-7.69(2 \mathrm{H}, \mathrm{m}, \operatorname{ArH}), 7.21(1 \mathrm{H}, \mathrm{t}, \mathrm{J}=7.5 \mathrm{~Hz}, \operatorname{ArH}), 7.10(1 \mathrm{H}$, d, J = 7.5 Hz, ArH), $7.03-6.99(2 \mathrm{H}, \mathrm{m}, \operatorname{ArH}), 5.87(1 \mathrm{H}, \mathrm{dd}, J=2.8,8.3 \mathrm{~Hz}, \mathrm{NH}), 4.21(1 \mathrm{H}$, dd, $J=3.5,9.2 \mathrm{~Hz}, \operatorname{ArCH}), 3.42-3.36(1 \mathrm{H}, \mathrm{m}, \mathrm{CHH}), 3.15\left(3 \mathrm{H}, \mathrm{s}, \mathrm{OCH}_{3}\right), 3.15-3.09(1 \mathrm{H}$, m, $\mathrm{CHH}), 2.32\left(3 \mathrm{H}, \mathrm{s}, \mathrm{ArCH}_{3}\right) ; \delta_{\mathrm{C}}\left(125 \mathrm{MHz}, \mathrm{CDCl}_{3}\right): 148.0$ (C), 138.5 (C), 138.0 (C), 134.0 (C), $133.4(\mathrm{CH}), 132.8(\mathrm{CH}), 131.0(\mathrm{CH}), 129.3(\mathrm{CH}), 128.7(\mathrm{CH}), 127.2(\mathrm{CH}), 125.4(\mathrm{CH})$, $123.6(\mathrm{CH}), 82.0(\mathrm{CH}), 56.8\left(\mathrm{CH}_{3}\right), 50.0\left(\mathrm{CH}_{2}\right), 21.4\left(\mathrm{CH}_{3}\right) ; \mathrm{m} / \mathrm{z}\left(\mathrm{ES}^{+}\right) 373[\mathrm{MNa}]^{+} ; \mathrm{HRMS}$ $\left(\mathrm{ES}^{+}\right)$calcd. for $\mathrm{C}_{16} \mathrm{H}_{18} \mathrm{~N}_{2} \mathrm{NaO}_{5} \mathrm{~S}[\mathrm{MNa}]^{+}$: 373.0829, found: 373.0830 .<smiles>CCOC(CNS(=O)(=O)c1ccccc1[N+](=O)[O-])c1cccc(C)c1</smiles>

$\mathrm{N}$-[2-Ethoxy-2-(m-tolyl)ethyl]-2-nitrobenzenesulfonamide $3 \mathrm{j}$. Using 1c (47 mg, $0.102 \mathrm{mmol})$ to make solution $\mathrm{A}$; 3methylstyrene $(60 \mathrm{mg}, 0.508 \mathrm{mmol})$ and $(\mathrm{pyr})_{4} \mathrm{Cu}(\mathrm{OTf})_{2}(7 \mathrm{mg}$, $0.010 \mathrm{mmol})$ to make solution $\mathrm{B} ; \mathrm{H}_{2} \mathrm{SO}_{4}(15 \mathrm{mg}, 0.153 \mathrm{mmol})$ in $\mathrm{EtOH}(4.0 \mathrm{ml})$ to make solution C, 3j (29 mg, 0.080 mmol, 78\%) was obtained as a clear oil. IR (film): $3346(\mathrm{~N}-\mathrm{H})$, $1593(\mathrm{C}=\mathrm{C}), 1361(\mathrm{~S}=\mathrm{O}), 1169(\mathrm{~S}=\mathrm{O}) \mathrm{cm}^{-1} ; \delta_{\mathrm{H}}\left(500 \mathrm{MHz}, \mathrm{CDCl}_{3}\right): 8.12-8.06(1 \mathrm{H}, \mathrm{m}, \mathrm{ArH})$, $7.92-7.86(1 \mathrm{H}, \mathrm{m}, \mathrm{ArH}), 7.76-7.69(2 \mathrm{H}, \mathrm{m}, \operatorname{ArH}), 7.20(1 \mathrm{H}, \mathrm{t}, J=7.4 \mathrm{~Hz}, \operatorname{ArH}), 7.09(1 \mathrm{H}$, d, $J=7.4 \mathrm{~Hz}, \operatorname{Ar} H), 7.04-7.00(2 \mathrm{H}, \mathrm{m}, \operatorname{ArH}), 5.93(1 \mathrm{H}, \mathrm{dd}, J=3.5,9.3 \mathrm{~Hz}, \mathrm{NH}), 4.32(1 \mathrm{H}$, 
$\mathrm{dd}, J=3.5,9.3 \mathrm{~Hz}, \mathrm{ArCH}), 3.43-3.35(2 \mathrm{H}, \mathrm{m}, \mathrm{OCH} H$ and $\mathrm{CH} H), 3.26-3.18(1 \mathrm{H}, \mathrm{m}$, $\mathrm{OCHH}), 3.12-3.06(1 \mathrm{H}, \mathrm{m}, \mathrm{CHH}), 2.32\left(3 \mathrm{H}, \mathrm{s}, \mathrm{ArCH}_{3}\right), 1.13\left(3 \mathrm{H}, \mathrm{t}, J=7.0 \mathrm{~Hz}, \mathrm{OCH}_{2} \mathrm{CH}_{3}\right)$; $\delta_{\mathrm{C}}\left(125 \mathrm{MHz}, \mathrm{CDCl}_{3}\right): 148.1(\mathrm{C}), 138.6(\mathrm{C}), 138.4(\mathrm{C}), 133.9(\mathrm{C}), 133.5(\mathrm{CH}), 132.7(\mathrm{CH})$, $131.0(\mathrm{CH}), 129.1(\mathrm{CH}), 128.6(\mathrm{CH}), 127.1(\mathrm{CH}), 125.4(\mathrm{CH}), 123.5(\mathrm{CH}), 80.0(\mathrm{CH}), 64.6$ $\left(\mathrm{CH}_{2}\right), 50.1\left(\mathrm{CH}_{2}\right), 21.4\left(\mathrm{CH}_{3}\right), 15.1\left(\mathrm{CH}_{3}\right) ; \mathrm{m} / \mathrm{z}\left(\mathrm{ES}^{+}\right) 387$ [MNa] $]^{+}$HRMS (ES $)$calcd. for $\mathrm{C}_{17} \mathrm{H}_{20} \mathrm{~N}_{2} \mathrm{NaO}_{5} \mathrm{~S}[\mathrm{MNa}]^{+}: 387.0985$, found: 387.0983 .<smiles>COC(CNS(=O)(=O)c1ccccc1[N+](=O)[O-])c1ccc(C)cc1</smiles>

3k

\section{$\mathrm{N}$-[2-Methoxy-2-(p-tolyl)ethyl]-2-nitrobenzenesulfonamide}

3k. Using 1c (47 mg, $0.102 \mathrm{mmol})$ to make solution $\mathrm{A}$; 4-

methylstyrene $(60 \mathrm{mg}, 0.508 \mathrm{mmol})$ and $(\mathrm{pyr})_{4} \mathrm{Cu}(\mathrm{OTf})_{2}(7$ $\mathrm{mg}, 0.010 \mathrm{mmol})$ to make solution $\mathrm{B} ; \mathrm{H}_{2} \mathrm{SO}_{4}(15 \mathrm{mg}, 0.153 \mathrm{mmol})$ in $\mathrm{MeOH}(4.0 \mathrm{ml})$ to make solution C, 3k (26 mg, $0.074 \mathrm{mmol}, 73 \%$ ) was obtained as a clear oil. IR (film): $3345(\mathrm{~N}-\mathrm{H})$, $1593(\mathrm{C}=\mathrm{C}), 1344(\mathrm{~S}=\mathrm{O}), 1167(\mathrm{~S}=\mathrm{O}) \mathrm{cm}^{-1} ; \delta_{\mathrm{H}}\left(500 \mathrm{MHz}, \mathrm{CDCl}_{3}\right): 8.10-8.05(1 \mathrm{H}, \mathrm{m}, \mathrm{ArH})$, $7.90-7.86(1 \mathrm{H}, \mathrm{m}, \operatorname{ArH}), 7.75-7.68(2 \mathrm{H}, \mathrm{m}, \operatorname{ArH}), 7.13(2 \mathrm{H}, \mathrm{d}, J=8.2 \mathrm{~Hz}, \operatorname{ArH}), 7.09(2 \mathrm{H}$, $\mathrm{d}, J=8.2 \mathrm{~Hz}, \operatorname{ArH}), 5.86(1 \mathrm{H}, \mathrm{dd}, J=3.0,8.3 \mathrm{~Hz}, \mathrm{NH}), 4.21(1 \mathrm{H}, \mathrm{dd}, J=3.6,9.1 \mathrm{~Hz}, \operatorname{ArCH})$, $3.41-3.35(1 \mathrm{H}, \mathrm{m}, \mathrm{CH}), 3.16-3.09(1 \mathrm{H}, \mathrm{m}, \mathrm{CHH}), 3.14\left(3 \mathrm{H}, \mathrm{s}, \mathrm{OCH}_{3}\right), 2.33(3 \mathrm{H}, \mathrm{s}$, $\left.\mathrm{ArCH}_{3}\right) ; \delta_{\mathrm{C}}\left(125 \mathrm{MHz}, \mathrm{CDCl}_{3}\right): 148.0(\mathrm{C}), 138.3$ (C), $135.0(\mathrm{C}), 134.0(\mathrm{C}), 133.4(\mathrm{CH}), 132.8$ $(\mathrm{CH}), 131.0(\mathrm{CH}), 129.4(\mathrm{CH}), 126.5(\mathrm{CH}), 125.4(\mathrm{CH}), 81.7(\mathrm{CH}), 56.7\left(\mathrm{CH}_{3}\right), 50.0\left(\mathrm{CH}_{2}\right)$, $21.2\left(\mathrm{CH}_{3}\right) ; \mathrm{m} / \mathrm{z}\left(\mathrm{ES}^{+}\right) 373[\mathrm{MNa}]^{+} ; \mathrm{HRMS}\left(\mathrm{ES}^{+}\right)$calcd. for $\mathrm{C}_{16} \mathrm{H}_{18} \mathrm{~N}_{2} \mathrm{NaO}_{5} \mathrm{~S}[\mathrm{MNa}]^{+}:$373.0829, found: 373.0830 .<smiles>Cc1ccc(C(Cl)CNS(=O)(=O)c2ccccc2[N+](=O)[O-])cc1</smiles>
3!

\section{$\mathrm{N}$-[2-Chloro-2-(p-tolyl)ethyl]-2-nitrobenzenesulfonamide $3 \mathrm{l}$.}

Using 1c (47 mg, $0.102 \mathrm{mmol}$ ) to make solution $\mathrm{A}$; 4methylstyrene $(60 \mathrm{mg}, 0.508 \mathrm{mmol})$ and $(\mathrm{pyr})_{4} \mathrm{Cu}(\mathrm{OTf})_{2}(7 \mathrm{mg}$, $0.010 \mathrm{mmol})$ to make solution $\mathrm{B}$; $\mathrm{HCl}\left(2 \mathrm{M}\right.$ in $\left.\mathrm{Et}_{2} \mathrm{O}, 1.0 \mathrm{ml}, 2.0 \mathrm{mmol}\right)$ in $\mathrm{CH}_{2} \mathrm{Cl}_{2}(3.0 \mathrm{ml})$ to make solution C, 3I (21 mg, $0.059 \mathrm{mmol}, 58 \%)$ was obtained as a clear oil. IR (film): 3342 $(\mathrm{N}-\mathrm{H}), 1593(\mathrm{C}=\mathrm{C}), 1348(\mathrm{~S}=\mathrm{O}), 1166(\mathrm{~S}=\mathrm{O}) \mathrm{cm}^{-1} ; \delta_{\mathrm{H}}\left(500 \mathrm{MHz}, \mathrm{CDCl}_{3}\right): 8.13-8.08(1 \mathrm{H}$, m, ArH), $7.92-7.87(1 \mathrm{H}, \mathrm{m}, \operatorname{ArH}), 7.78-7.72(2 \mathrm{H}, \mathrm{m}, \operatorname{ArH}), 7.18(2 \mathrm{H}, \mathrm{d}, J=8.2 \mathrm{~Hz}, \operatorname{Ar} H)$, 
$7.13(2 \mathrm{H}, \mathrm{d}, J=8.2 \mathrm{~Hz}, \operatorname{ArH}), 5.80-5.67(1 \mathrm{H}, \mathrm{m}, \mathrm{NH}), 4.92(1 \mathrm{H}, \mathrm{dd}, J=5.9,8.0 \mathrm{~Hz}, \operatorname{ArCH})$, $3.71-3.64(1 \mathrm{H}, \mathrm{m}, \mathrm{CH}), 3.63-3.56(1 \mathrm{H}, \mathrm{m}, \mathrm{CHH}), 2.33\left(3 \mathrm{H}, \mathrm{s}, \mathrm{ArCH}_{3}\right) ; \delta_{\mathrm{c}}(125 \mathrm{MHz}$, $\left.\mathrm{CDCl}_{3}\right): 147.9(\mathrm{C}), 139.2(\mathrm{C}), 134.5(\mathrm{C}), 134.0(\mathrm{C}), 133.7(\mathrm{CH}), 133.0(\mathrm{CH}), 130.7(\mathrm{CH})$, $129.6(\mathrm{CH}), 127.0(\mathrm{CH}), 125.6(\mathrm{CH}), 61.3(\mathrm{CH}), 51.0\left(\mathrm{CH}_{2}\right), 21.2\left(\mathrm{CH}_{3}\right) ; \mathrm{m} / \mathrm{z}\left(\mathrm{ES}^{+}\right) 377$ [MNa] ${ }^{+}$; HRMS $\left(\mathrm{ES}^{+}\right)$calcd. for $\mathrm{C}_{15} \mathrm{H}_{15} \mathrm{CIN}_{2} \mathrm{NaO}_{4} \mathrm{~S}$ [MNa] $]^{+}: 377.0333$, found: 377.0334 .<smiles>COC(CNS(=O)(=O)c1ccccc1[N+](=O)[O-])c1ccc(Cl)cc1</smiles>

$3 m$

\section{$\mathrm{N}$-[2-(4-Chlorophenyl)-2-methoxyethyl]-2-nitrobenzene}

sulfonamide $3 \mathrm{~m}$. Using $1 \mathrm{c}(47 \mathrm{mg}, 0.102 \mathrm{mmol})$ to make solution $\mathrm{A}$; 4-chlorostyrene $(70 \mathrm{mg}, 0.505 \mathrm{mmol})$ and (pyr) ${ }_{4} \mathrm{Cu}(\mathrm{OTf})_{2}(7 \mathrm{mg}, 0.010 \mathrm{mmol})$ to make solution $\mathrm{B} ; \mathrm{H}_{2} \mathrm{SO}_{4}(15 \mathrm{mg}, 0.153 \mathrm{mmol})$ in $\mathrm{MeOH}$ $(4.0 \mathrm{ml})$ to make solution C, $3 \mathrm{~m}(30 \mathrm{mg}, 0.081 \mathrm{mmol}, 79 \%)$ was obtained as a clear oil. IR (film): $3344(\mathrm{~N}-\mathrm{H}), 1595(\mathrm{C}=\mathrm{C}), 1345(\mathrm{~S}=\mathrm{O}), 1166(\mathrm{~S}=\mathrm{O}) \mathrm{cm}^{-1} ; \delta_{\mathrm{H}}\left(500 \mathrm{MHz}, \mathrm{CDCl}_{3}\right): 8.06-$ $8.02(1 \mathrm{H}, \mathrm{m}, \operatorname{ArH}), 7.90-7.86(1 \mathrm{H}, \mathrm{m}, \operatorname{ArH}), 7.76-7.68(2 \mathrm{H}, \mathrm{m}, \operatorname{Ar} H), 7.27(2 \mathrm{H}, \mathrm{d}, J=8.4$ $\mathrm{Hz}, \operatorname{ArH}), 7.16(2 \mathrm{H}, \mathrm{d}, J=8.4 \mathrm{~Hz}, \operatorname{ArH}), 5.85(1 \mathrm{H}, \mathrm{dd}, J=3.5,8.0 \mathrm{~Hz}, \mathrm{NH}), 4.24(1 \mathrm{H}, \mathrm{dd}, J=$ 3.6, $8.7 \mathrm{~Hz}, \mathrm{ArCH}), 3.44-3.36(1 \mathrm{H}, \mathrm{m}, \mathrm{CH}), 3.16\left(3 \mathrm{H}, \mathrm{s}, \mathrm{OCH}_{3}\right), 3.16-3.09(1 \mathrm{H}, \mathrm{m}$, $\mathrm{CHH}) ; \delta_{\mathrm{C}}\left(125 \mathrm{MHz}, \mathrm{CDCl}_{3}\right): 148.0(\mathrm{C}), 136.6(\mathrm{C}), 134.3(\mathrm{C}), 134.0(\mathrm{C}), 133.5(\mathrm{CH}), 132.8$ $(\mathrm{CH}), 130.9(\mathrm{CH}), 128.9(\mathrm{CH}), 127.9(\mathrm{CH}), 125.4(\mathrm{CH}), 81.4(\mathrm{CH}), 56.9\left(\mathrm{CH}_{3}\right), 49.8\left(\mathrm{CH}_{2}\right)$; $\mathrm{m} / \mathrm{z}\left(\mathrm{ES}^{+}\right) 393$ [MNa] $^{+}$; HRMS $\left(\mathrm{ES}^{+}\right)$calcd. for $\mathrm{C}_{15} \mathrm{H}_{15} \mathrm{CIN}_{2} \mathrm{NaO}_{5} \mathrm{~S}[\mathrm{MNa}]^{+}:$393.0282, found: 393.0284 .<smiles>O=[N+]([O-])c1ccccc1S(=O)(=O)NCC(OCc1ccccc1)c1ccc(Cl)cc1</smiles>

solution C, 3n (28 mg, $0.063 \mathrm{mmol}, 61 \%)$ was obtained as a clear oil. IR (film): $3362(\mathrm{~N}-\mathrm{H})$, $1595(\mathrm{C}=\mathrm{C}), 1345(\mathrm{~S}=\mathrm{O}), 1168(\mathrm{~S}=\mathrm{O}) \mathrm{cm}^{-1} ; \delta_{\mathrm{H}}\left(500 \mathrm{MHz}, \mathrm{CDCl}_{3}\right): 8.03-7.98(1 \mathrm{H}, \mathrm{m}, \mathrm{ArH})$, $7.85-7.80(1 \mathrm{H}, \mathrm{m}, \mathrm{ArH}), 7.72-7.63(2 \mathrm{H}, \mathrm{m}, \mathrm{ArH}), 7.37-7.28(5 \mathrm{H}, \mathrm{m}, \mathrm{ArH}), 7.25-7.19$ 
$(4 \mathrm{H}, \mathrm{m}, \operatorname{ArH}), 5.87(1 \mathrm{H}, \mathrm{dd}, J=3.7,8.0 \mathrm{~Hz}, \mathrm{NH}), 4.48(1 \mathrm{H}, \mathrm{dd}, J=3.7,8.5 \mathrm{~Hz}, \operatorname{ArCH}), 4.41$ $(1 \mathrm{H}, \mathrm{d}, J=11.4 \mathrm{~Hz}, \mathrm{OCH} H), 4.22(1 \mathrm{H}, \mathrm{d}, J=11.4 \mathrm{~Hz}, \mathrm{OCHH}), 3.47-3.41(1 \mathrm{H}, \mathrm{m}, \mathrm{CH} H)$, 3.24 - $3.18(1 \mathrm{H}, \mathrm{m}, \mathrm{CHH}) ; \delta_{\mathrm{C}}\left(125 \mathrm{MHz}, \mathrm{CDCl}_{3}\right)$ : $148.0(\mathrm{C}), 137.0(\mathrm{C}), 136.7(\mathrm{C}), 134.4(\mathrm{C})$, $133.9(\mathrm{C}), 133.4(\mathrm{CH}), 132.8(\mathrm{CH}), 130.7(\mathrm{CH}), 129.0(\mathrm{CH}), 128.6(\mathrm{CH}), 128.10(\mathrm{CH}), 128.09$ $(\mathrm{CH}), 127.9(\mathrm{CH}), 125.4(\mathrm{CH}), 78.9(\mathrm{CH}), 70.9\left(\mathrm{CH}_{2}\right), 49.9\left(\mathrm{CH}_{2}\right) ; \mathrm{m} / \mathrm{z}\left(\mathrm{ES}^{+}\right) 469[\mathrm{MNa}]^{+}$; HRMS $\left(\right.$ ES $\left.^{+}\right)$calcd. for $\mathrm{C}_{21} \mathrm{H}_{19} \mathrm{CIN}_{2} \mathrm{NaO}_{5} \mathrm{~S}[\mathrm{MNa}]^{+}: 469.0595$, found: 469.0599 .

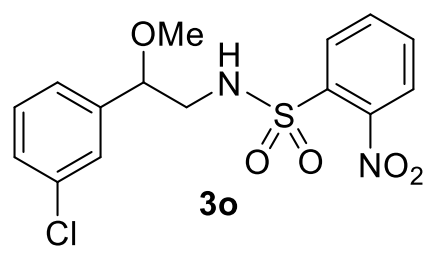

\section{$\mathrm{N}$-[2-(3-Chlorophenyl)-2-methoxyethyl]-2-nitrobenzenesulfon} amide 3o. Using 1c (47 mg, $0.102 \mathrm{mmol})$ to make solution $\mathrm{A}$; 3chlorostyrene $(70 \mathrm{mg}, 0.505 \mathrm{mmol})$ and $(\mathrm{pyr})_{4} \mathrm{Cu}(\mathrm{OTf})_{2}(7 \mathrm{mg}$, $0.010 \mathrm{mmol})$ to make solution $\mathrm{B} ; \mathrm{H}_{2} \mathrm{SO}_{4}(30 \mathrm{mg}, 0.306 \mathrm{mmol})$ in $\mathrm{MeOH}(4.0 \mathrm{ml})$ to make solution C, 30 (18 mg, $0.049 \mathrm{mmol}, 48 \%$ ) was obtained as a clear oil. IR (film): $3347(\mathrm{~N}-\mathrm{H})$, $1595(\mathrm{C}=\mathrm{C}), 1345(\mathrm{~S}=\mathrm{O}), 1165(\mathrm{~S}=\mathrm{O}) \mathrm{cm}^{-1} ; \delta_{\mathrm{H}}\left(500 \mathrm{MHz}, \mathrm{CDCl}_{3}\right): 8.10-8.05(1 \mathrm{H}, \mathrm{m}, \mathrm{ArH})$, $7.93-7.89(1 \mathrm{H}, \mathrm{m}, \mathrm{ArH}), 7.81-7.75(2 \mathrm{H}, \mathrm{m}, \operatorname{ArH}), 7.34-7.29(2 \mathrm{H}, \mathrm{m}, \operatorname{ArH}), 7.27-7.24$ $(1 \mathrm{H}, \mathrm{m}, \operatorname{ArH}), 7.20-7.15(1 \mathrm{H}, \mathrm{m}, \operatorname{ArH}), 5.83(1 \mathrm{H}, \mathrm{dd}, J=3.4,7.5 \mathrm{~Hz}, \mathrm{NH}), 4.26(1 \mathrm{H}, \mathrm{dd}, J=$ 3.6, $8.7 \mathrm{~Hz}, \mathrm{ArCH}), 3.48-3.41(1 \mathrm{H}, \mathrm{m}, \mathrm{CH}), 3.22-3.15(1 \mathrm{H}, \mathrm{m}, \mathrm{CHH}), 3.19(3 \mathrm{H}, \mathrm{s}$, $\left.\mathrm{OCH}_{3}\right) ; \delta_{\mathrm{C}}\left(125 \mathrm{MHz}, \mathrm{CDCl}_{3}\right): 147.9(\mathrm{C}), 140.5(\mathrm{C}), 134.5(\mathrm{C}), 133.7(\mathrm{CH}), 133.6(\mathrm{C}), 132.9$ $(\mathrm{CH}), 130.8(\mathrm{CH}), 130.0(\mathrm{CH}), 128.5(\mathrm{CH}), 126.6(\mathrm{CH}), 125.4(\mathrm{CH}), 125.0(\mathrm{CH}), 81.2(\mathrm{CH})$, $56.9\left(\mathrm{CH}_{3}\right), 49.7\left(\mathrm{CH}_{2}\right) ; \mathrm{m} / \mathrm{z}\left(\mathrm{ES}^{+}\right) 393[\mathrm{MNa}]^{+}$; HRMS $\left(\mathrm{ES}^{+}\right)$calcd. for $\mathrm{C}_{15} \mathrm{H}_{15} \mathrm{ClN}_{2} \mathrm{NaO}_{5} \mathrm{~S}$ $[\mathrm{MNa}]^{+}:$393.0282, found: 393.0284.

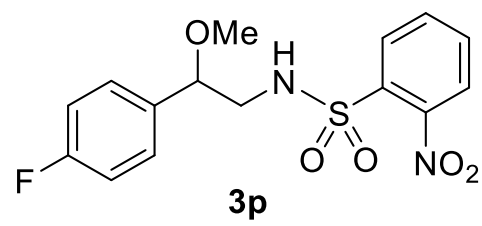

\section{N-[2-(4-Fluorophenyl)-2-methoxyethyl]-2-nitrobenzene} sulfonamide $3 \mathrm{p}$. Using 1c $(47 \mathrm{mg}, 0.102 \mathrm{mmol})$ to make solution $\mathrm{A}$; 4-fluorostyrene (62 $\mathrm{mg}, 0.508 \mathrm{mmol})$ and (pyr $)_{4} \mathrm{Cu}(\mathrm{OTf})_{2}(7 \mathrm{mg}, 0.010 \mathrm{mmol})$ to make solution $\mathrm{B} ; \mathrm{H}_{2} \mathrm{SO}_{4}(15 \mathrm{mg}, 0.153 \mathrm{mmol})$ in $\mathrm{MeOH}$ $(4.0 \mathrm{ml})$ to make solution C, $3 \mathrm{p}(28 \mathrm{mg}, 0.079 \mathrm{mmol}, 77 \%)$ was obtained as a clear oil. IR (film): $3345(\mathrm{~N}-\mathrm{H}), 1605$ (C=C), $1344(\mathrm{~S}=\mathrm{O}), 1114(\mathrm{~S}=\mathrm{O}) \mathrm{cm}^{-1} ; \delta_{\mathrm{H}}\left(500 \mathrm{MHz}, \mathrm{CDCl}_{3}\right): 8.09-$ $8.05(1 \mathrm{H}, \mathrm{m}, \operatorname{ArH}), 7.91-7.86(1 \mathrm{H}, \mathrm{m}, \operatorname{ArH}), 7.76-7.69(2 \mathrm{H}, \mathrm{m}, \operatorname{ArH}), 7.20(2 \mathrm{H}, \mathrm{dd}, J=5.4$, 
8.6 Hz, ArH), $7.01(2 \mathrm{H}, \mathrm{t}, J=8.6 \mathrm{~Hz}, \operatorname{ArH}), 5.85(1 \mathrm{H}, \mathrm{dd}, J=3.1,8.3 \mathrm{~Hz}, \mathrm{NH}), 4.24(1 \mathrm{H}, \mathrm{dd}, J$ $=3.7,8.9 \mathrm{~Hz}, \mathrm{ArCH}), 3.41-3.35(1 \mathrm{H}, \mathrm{m}, \mathrm{CH}), 3.15\left(3 \mathrm{H}, \mathrm{s}, \mathrm{OCH}_{3}\right), 3.14-3.09(1 \mathrm{H}, \mathrm{m}$, $\mathrm{CHH}) ; \delta_{\mathrm{C}}\left(125 \mathrm{MHz}, \mathrm{CDCl}_{3}\right): 162.7$ (d, $\left.J=249.0 \mathrm{~Hz},(\mathrm{CF})\right), 148.0(\mathrm{C}), 134.0(\mathrm{C}), 133.8$ (d, J = $3.0 \mathrm{~Hz},(\mathrm{C})), 133.5(\mathrm{CH}), 132.8(\mathrm{CH}), 130.9(\mathrm{CH}), 128.3(\mathrm{~d}, \mathrm{~J}=8.3 \mathrm{~Hz},(\mathrm{CH})), 125.4(\mathrm{CH})$, $115.7(\mathrm{~d}, J=22.1 \mathrm{~Hz},(\mathrm{CH})), 81.3(\mathrm{CH}), 56.8\left(\mathrm{CH}_{3}\right), 49.9\left(\mathrm{CH}_{2}\right) ; \mathrm{m} / \mathrm{z}\left(\mathrm{ES}^{+}\right) 377[\mathrm{MNa}]^{+}$; HRMS $\left(\mathrm{ES}^{+}\right)$calcd. for $\mathrm{C}_{15} \mathrm{H}_{15} \mathrm{FN}_{2} \mathrm{NaO}_{5} \mathrm{~S}[\mathrm{MNa}]^{+}: 377.0578$, found: 377.0574 .<smiles>O=[N+]([O-])c1ccccc1S(=O)(=O)NCC(Cl)c1ccc(F)cc1</smiles>

$\mathrm{mg}, 0.010 \mathrm{mmol})$ to make solution $\mathrm{B}$; $\mathrm{HCl}\left(2 \mathrm{M}\right.$ in $\left.\mathrm{Et}_{2} \mathrm{O}, 1.0 \mathrm{ml}, 2.0 \mathrm{mmol}\right)$ in $\mathrm{CH}_{2} \mathrm{Cl}_{2}(3.0 \mathrm{ml})$ to make solution C, 3q (19 mg, $0.053 \mathrm{mmol}, 52 \%)$ was obtained as a clear oil. IR (film): 3356 $(\mathrm{N}-\mathrm{H}), 1605(\mathrm{C}=\mathrm{C}), 1348(\mathrm{~S}=\mathrm{O}), 1165(\mathrm{~S}=\mathrm{O}) \mathrm{cm}^{-1} ; \delta_{\mathrm{H}}\left(500 \mathrm{MHz}, \mathrm{CDCl}_{3}\right): 8.11-8.07(1 \mathrm{H}$, m, $\operatorname{Ar} H), 7.91-7.88(1 \mathrm{H}, \mathrm{m}, \operatorname{Ar} H), 7.78-7.73(2 \mathrm{H}, \mathrm{m}, \operatorname{ArH}), 7.31(2 \mathrm{H}, \mathrm{dd}, J=5.1,8.6 \mathrm{~Hz}$, $\operatorname{ArH}), 7.02(2 \mathrm{H}, \mathrm{t}, J=8.6 \mathrm{~Hz}, \operatorname{ArH}), 5.81-5.70(1 \mathrm{H}, \mathrm{m}, \mathrm{NH}), 4.96(1 \mathrm{H}, \mathrm{dd}, J=6.0,7.9 \mathrm{~Hz}$, $\operatorname{ArCH}), 3.70-3.63(1 \mathrm{H}, \mathrm{m}, \mathrm{CHH}), 3.62-3.56(1 \mathrm{H}, \mathrm{m}, \mathrm{CHH}) ; \delta_{\mathrm{C}}\left(125 \mathrm{MHz}, \mathrm{CDCl}_{3}\right): 162.9(\mathrm{~d}$, $J=249.0 \mathrm{~Hz},(\mathrm{CF})), 147.9(\mathrm{C}), 134.0(\mathrm{C}), 133.8(\mathrm{CH}), 133.5(\mathrm{~d}, J=4.0 \mathrm{~Hz},(\mathrm{C})), 133.1(\mathrm{CH})$, $130.7(\mathrm{CH}), 129.1(\mathrm{~d}, J=8.0 \mathrm{~Hz},(\mathrm{CH})), 125.6(\mathrm{CH}), 116.9(\mathrm{~d}, J=21.1 \mathrm{~Hz},(\mathrm{CH})), 60.7(\mathrm{CH})$, $51.1\left(\mathrm{CH}_{2}\right) ; \mathrm{m} / \mathrm{z}\left(\mathrm{ES}^{+}\right) 381[\mathrm{MNa}]^{+} ; \mathrm{HRMS}\left(\mathrm{ES}^{+}\right)$calcd. for $\mathrm{C}_{14} \mathrm{H}_{12} \mathrm{ClFN}_{2} \mathrm{NaO}_{4} \mathrm{~S}[\mathrm{MNa}]^{+}$: 381.0083 , found: 381.0074 .

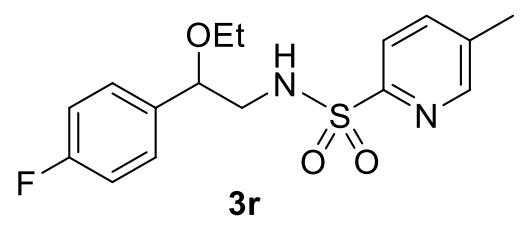

\section{N-[2-Ethoxy-2-(4-fluorophenyl)ethyl]-5-methylpyridine-}

2- sulfonamide $3 \mathrm{r}$. Using $1 \mathrm{~d}$ (44 $\mathrm{mg}, \mathrm{mmol}, 0.102 \mathrm{mmol})$ to make solution A; 4-fluorostyrene (62 $\mathrm{mg}, 0.508 \mathrm{mmol})$ and $(\text { pyr })_{4} \mathrm{Cu}(\mathrm{OTf})_{2}(7 \mathrm{mg}, 0.010 \mathrm{mmol})$ to make solution $\mathrm{B} ; \mathrm{H}_{2} \mathrm{SO}_{4}(15 \mathrm{mg}, 0.153 \mathrm{mmol})$ in $\mathrm{EtOH}$ $(4.0 \mathrm{ml})$ to make solution $\mathrm{C}, 3 \mathrm{r}(21 \mathrm{mg}, 0.062 \mathrm{mmol}, 61 \%)$ was obtained as a clear oil. IR (film): $3275(\mathrm{~N}-\mathrm{H}), 1604(\mathrm{C}=\mathrm{C}), 1332(\mathrm{~S}=\mathrm{O}), 1171(\mathrm{~S}=\mathrm{O}) \mathrm{cm}^{-1} ; \delta_{\mathrm{H}}\left(500 \mathrm{MHz}, \mathrm{CDCl}_{3}\right): 8.54-$ $8.50(1 \mathrm{H}, \mathrm{m}, \mathrm{ArH}), 7.88-7.84(1 \mathrm{H}, \mathrm{m}, \operatorname{ArH}), 7.70-7.65(1 \mathrm{H}, \mathrm{m}, \operatorname{Ar} H), 7.21(2 \mathrm{H}, \mathrm{dd}, J=5.6$, 
$8.5 \mathrm{~Hz}, \operatorname{ArH}), 7.00(2 \mathrm{H}, \mathrm{t}, J=8.5 \mathrm{~Hz}, \operatorname{ArH}), 5.32(1 \mathrm{H}, \mathrm{dd}, J=3.2,9.1 \mathrm{~Hz}, \mathrm{NH}), 4.35(1 \mathrm{H}, \mathrm{dd}, J$ $=3.8,9.2 \mathrm{~Hz}, \mathrm{ArCH}), 3.40-3.24\left(3 \mathrm{H}, \mathrm{m}, \mathrm{OCH}_{2}\right.$ and $\left.\mathrm{CHH}\right), 3.10-3.03(1 \mathrm{H}, \mathrm{m}, \mathrm{CHH}), 2.43$ $\left(3 \mathrm{H}, \mathrm{s}, \mathrm{ArCH}_{3}\right), 1.15\left(3 \mathrm{H}, \mathrm{t}, J=7.0 \mathrm{~Hz}, \mathrm{OCH}_{2} \mathrm{CH}_{3}\right) ; \delta_{\mathrm{C}}\left(125 \mathrm{MHz}, \mathrm{CDCl}_{3}\right): 162.6(\mathrm{~d}, J=246.0$ Hz, (CF)), $154.9(\mathrm{C}), 150.6(\mathrm{CH}), 138.1(\mathrm{CH}), 137.2(\mathrm{C}), 134.8$ (d, J = 3.0 Hz, (C)), 128.2 (d, $J=8.0 \mathrm{~Hz},(\mathrm{CH})), 121.7(\mathrm{CH}), 115.5(\mathrm{~d}, J=22.1 \mathrm{~Hz},(\mathrm{CH})), 79.9(\mathrm{CH}), 64.5\left(\mathrm{CH}_{2}\right), 49.8$ $\left(\mathrm{CH}_{2}\right), \quad 18.5\left(\mathrm{CH}_{3}\right), 15.2 \quad\left(\mathrm{CH}_{3}\right) ; \quad \mathrm{m} / \mathrm{z} \quad\left(\mathrm{ES}^{+}\right) \quad 361 \quad[\mathrm{MNa}]^{+} ;$HRMS (ES $)$calcd. for $\mathrm{C}_{16} \mathrm{H}_{19} \mathrm{FN}_{2} \mathrm{NaO}_{3} \mathrm{~S}[\mathrm{MNa}]^{+}: 361.0993$, found: 361.0993. 


\section{Flow synthesis of imidazolines $4 a-c$ by telescoped aziridination/ring-expansion}

The same tube reactor used in Section 6 was used for the flow synthesis of imidazolines 4ac. $1 \mathrm{~d}(0.10 \mathrm{mmol}, 1.0 \mathrm{eq})$ was dissolved in $\mathrm{CH}_{2} \mathrm{Cl}_{2}(2.0 \mathrm{ml})$ to give solution A. Alkene $(0.50$ $\mathrm{mmol}, 5.0 \mathrm{eq})$ and $(\mathrm{pyr})_{4} \mathrm{Cu}(\mathrm{OTf})_{2}(0.01 \mathrm{mmol}, 0.1 \mathrm{eq})$ were dissolved in anhydrous $\mathrm{MeCN}$ $(2.0 \mathrm{ml})$ to give solution $\mathrm{B}$. Boron trifluoride $\left(15 \% \mathrm{BF}_{3}\right.$ in $\left.\mathrm{MeCN}, 0.5 \mathrm{mmol}, 5 \mathrm{eq}\right)$ was dissolved in anhydrous $\mathrm{MeCN}(4.0 \mathrm{ml})$ to make solution $\mathrm{C}$. Solutions $\mathrm{A}$ and $\mathrm{B}$ were combined in the first section of the tube reactor at room temperature, with a residence time of 10 min. Solution $\mathrm{C}$ was then added in the second section of the tube reactor at room temperature, with a residence time of $10 \mathrm{~min}$. The outlet stream was quenched into saturated aq $\mathrm{NaHCO}_{3}(5 \mathrm{ml})$. Upon completion of the run, additional quantities of $\mathrm{CH}_{2} \mathrm{Cl}_{2}$ were passed through the tube reactor to ensure all the product was collected. The resulting mixture was concentrated under vacuum and purified by gradient column chromatography (3:1 ${ }^{n}$ hexane:EtOAc, then $1: 1^{n}$ hexane:EtOAc) to give the following products:

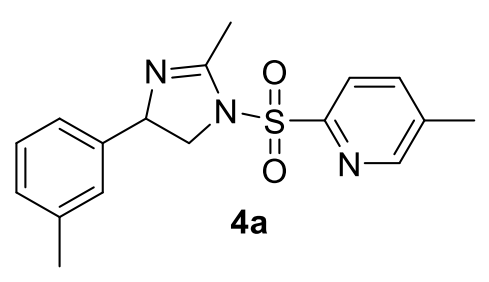

\section{5-Methyl-2-[[2-methyl-4-(m-tolyl)-4,5-dihydro-1H-imidazol-} 1-yl]sulfonyl]pyridine 4a. Using 1d $(44 \mathrm{mg}, \mathrm{mmol}, 0.102$ $\mathrm{mmol}$ ) to make solution $\mathrm{A}$; 3-methylstyrene $(60 \mathrm{mg}, 0.508$ $\mathrm{mmol})$ and $(\mathrm{pyr})_{4} \mathrm{Cu}(\mathrm{OTf})_{2}(7 \mathrm{mg}, 0.010 \mathrm{mmol})$ to make solution $\mathrm{B}$; and $\mathrm{BF}_{3}\left(0.23 \mathrm{~g}\right.$ of $15 \% \mathrm{BF}_{3}$ in $\mathrm{MeCN}$ solution; $\left.0.52 \mathrm{mmol}\right)$ in $\mathrm{MeCN}(4.0 \mathrm{ml})$ to make solution C, 4a (26 mg, $0.079 \mathrm{mmol}, 77 \%$ ) was obtained as a clear oil. IR (film): 1650, $1356(\mathrm{~S}=\mathrm{O}), 1174(\mathrm{~S}=\mathrm{O}) \mathrm{cm}^{-1} ; \delta_{\mathrm{H}}\left(500 \mathrm{MHz}, \mathrm{CDCl}_{3}\right): 8.55-8.51(1 \mathrm{H}, \mathrm{m}, \mathrm{ArH}), 7.92-7.87$ $(1 \mathrm{H}, \mathrm{m}, \operatorname{ArH}), 7.74-7.69(1 \mathrm{H}, \mathrm{m}, \operatorname{ArH}), 7.17(1 \mathrm{H}, \mathrm{t}, J=7.7 \mathrm{~Hz}, \operatorname{ArH}), 7.05(1 \mathrm{H}, \mathrm{d}, J=7.7 \mathrm{~Hz}$, $\operatorname{ArH}), 6.96-6.91(2 \mathrm{H}, \mathrm{m}, \mathrm{ArH}), 5.05-4.97(1 \mathrm{H}, \mathrm{m}, \mathrm{ArCH}), 4.25(1 \mathrm{H}, \mathrm{t}, J=10.0 \mathrm{~Hz}, \mathrm{CH} H)$, $3.69(1 \mathrm{H}, \mathrm{dd}, J=8.0,10.0 \mathrm{~Hz}, \mathrm{CHH}), 2.46\left(3 \mathrm{H}, \mathrm{s}, \mathrm{ArCH}_{3}\right), 2.45-2.44\left(3 \mathrm{H}, \mathrm{m}, \mathrm{CH}_{3}\right), 2.29$ (3H, s, $\left.\mathrm{ArCH}_{3}\right) ; \delta_{\mathrm{C}}\left(125 \mathrm{MHz}, \mathrm{CDCl}_{3}\right): 157.1(\mathrm{C}), 153.5$ (C), $150.8(\mathrm{CH}), 141.7(\mathrm{C}), 138.3(\mathrm{C})$, $138.2(\mathrm{CH}), 138.1(\mathrm{C}), 128.5(\mathrm{CH}), 128.3(\mathrm{CH}), 127.2(\mathrm{CH}), 123.7(\mathrm{CH}), 122.5(\mathrm{CH}), 67.0$ $(\mathrm{CH}), 55.9\left(\mathrm{CH}_{2}\right), 21.4\left(\mathrm{CH}_{3}\right), 18.6\left(\mathrm{CH}_{3}\right), 16.9\left(\mathrm{CH}_{3}\right) ; \mathrm{m} / \mathrm{z}\left(\mathrm{ES}^{+}\right) 330[\mathrm{MH}]^{+} ; \mathrm{HRMS}\left(\mathrm{ES}^{+}\right)$ calcd. for $\mathrm{C}_{17} \mathrm{H}_{20} \mathrm{~N}_{3} \mathrm{O}_{2} \mathrm{~S}[\mathrm{MH}]^{+}: 330.1271$, found: 330.1275 . 


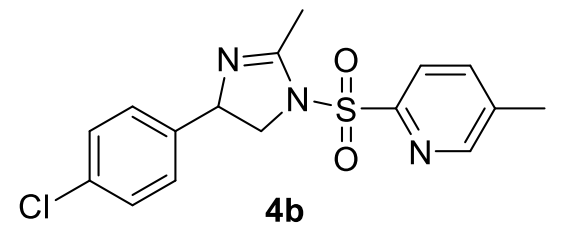

\section{5-methyl-2-[[4-(4-Chlorophenyl)-2-methyl-4,5-dihydro-1H} -imidazol-1-yl]sulfonyl]- pyridine 4b. Using 1d (44 mg,

$\mathrm{mmol}, 0.102 \mathrm{mmol}$ ) to make solution A; 4-chlorostyrene (70 $\mathrm{mg}, 0.505 \mathrm{mmol})$ and $(\mathrm{pyr})_{4} \mathrm{Cu}(\mathrm{OTf})_{2}(7 \mathrm{mg}, 0.010 \mathrm{mmol})$ to make solution $\mathrm{B} ; \mathrm{BF}_{3}(0.23 \mathrm{~g}$ of $15 \% \mathrm{BF}_{3}$ in $\mathrm{MeCN}$ solution; $\left.0.52 \mathrm{mmol}\right)$ in $\mathrm{MeCN}(4.0 \mathrm{ml})$ to make solution $\mathrm{C}, \mathbf{4 b}(25 \mathrm{mg}$, $0.071 \mathrm{mmol}, 70 \%$ ) was obtained as a clear oil. IR (film): 1650, $1356(\mathrm{~S}=0), 1172(\mathrm{~S}=\mathrm{O})$ $\mathrm{cm}^{-1} ; \delta_{\mathrm{H}}\left(500 \mathrm{MHz}, \mathrm{CDCl}_{3}\right): 8.52-8.48(1 \mathrm{H}, \mathrm{m}, \mathrm{ArH}), 7.91-7.87(1 \mathrm{H}, \mathrm{m}, \mathrm{ArH}), 7.74-7.69$ $(1 \mathrm{H}, \mathrm{m}, \operatorname{ArH}), 7.26(2 \mathrm{H}, \mathrm{d}, J=8.4 \mathrm{~Hz}, \operatorname{ArH}), 7.09(2 \mathrm{H}, \mathrm{d}, J=8.4 \mathrm{~Hz}, \operatorname{ArH}), 5.06-5.00(1 \mathrm{H}$, m, $\operatorname{ArCH}), 4.27(1 \mathrm{H}, \mathrm{t}, J=10.0 \mathrm{~Hz}, \mathrm{CHH}), 3.66(1 \mathrm{H}, \mathrm{dd}, J=7.9,10.0 \mathrm{~Hz}, \mathrm{CHH}), 2.46(3 \mathrm{H}, \mathrm{s}$, $\left.\mathrm{ArCH}_{3}\right), 2.44-2.42\left(3 \mathrm{H}, \mathrm{m}, \mathrm{CH}_{3}\right) ; \delta_{\mathrm{C}}\left(125 \mathrm{MHz}, \mathrm{CDCl}_{3}\right): 157.7(\mathrm{C}), 153.5(\mathrm{C}), 150.7(\mathrm{CH})$, $140.4(\mathrm{C}), 138.32(\mathrm{CH}), 138.29(\mathrm{C}), 133.4(\mathrm{C}), 128.8(\mathrm{CH}), 128.0(\mathrm{CH}), 122.4(\mathrm{CH}), 66.3$ (CH), $56.0\left(\mathrm{CH}_{2}\right), 18.6\left(\mathrm{CH}_{3}\right), 16.9\left(\mathrm{CH}_{3}\right) ; \mathrm{m} / \mathrm{z}\left(\mathrm{ES}^{+}\right) 350[\mathrm{MH}]^{+}$; HRMS (ES $)$calcd. for $\mathrm{C}_{16} \mathrm{H}_{17} \mathrm{CIN}_{3} \mathrm{O}_{2} \mathrm{~S}[\mathrm{MNa}]^{+}: 350.0725$, found: 350.0726 .
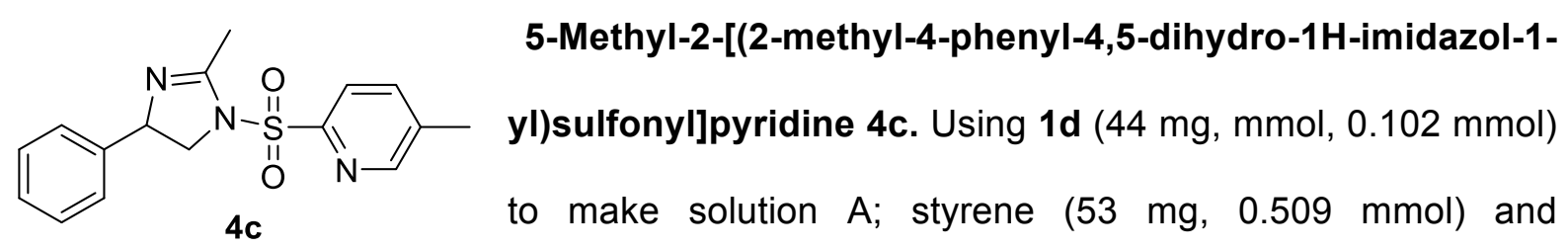

$(\text { pyr })_{4} \mathrm{Cu}(\mathrm{OTf})_{2}(7 \mathrm{mg}, 0.010 \mathrm{mmol})$ to make solution $\mathrm{B} ; \mathrm{BF}_{3}\left(0.23 \mathrm{~g}\right.$ of $15 \% \mathrm{BF}_{3}$ in $\mathrm{MeCN}$ solution; $0.52 \mathrm{mmol})$ in $\mathrm{MeCN}(4.0 \mathrm{ml})$ to make solution C, 4c $(23 \mathrm{mg}, 0.073 \mathrm{mmol}, 71 \%)$ was obtained as a clear oil. IR (film): 1651, $1356(\mathrm{~S}=\mathrm{O}), 1173(\mathrm{~S}=\mathrm{O}) \mathrm{cm}^{-1} ; \delta_{\mathrm{H}}(500 \mathrm{MHz}$, $\left.\mathrm{CDCl}_{3}\right): 8.55-8.49(1 \mathrm{H}, \mathrm{m}, \mathrm{ArH}), 7.93-7.86(1 \mathrm{H}, \mathrm{m}, \mathrm{ArH}), 7.75-7.68(1 \mathrm{H}, \mathrm{m}, \operatorname{ArH}), 7.31-$ $7.22(3 \mathrm{H}, \mathrm{m}, \operatorname{ArH}), 7.15(2 \mathrm{H}, \mathrm{d}, J=7.5 \mathrm{~Hz}, \operatorname{ArH}), 5.09-5.01(1 \mathrm{H}, \mathrm{m}, \operatorname{ArCH}), 4.27(1 \mathrm{H}, \mathrm{t}, J=$ $10.0 \mathrm{~Hz}, \mathrm{CHH}), 3.70(1 \mathrm{H}, \mathrm{dd}, J=8.1,10.0 \mathrm{~Hz}, \mathrm{CHH}), 2.46\left(3 \mathrm{H}, \mathrm{s}, \mathrm{ArCH}_{3}\right), 2.45-2.44(3 \mathrm{H}$, m, $\left.\mathrm{CH}_{3}\right) ; \delta_{\mathrm{C}}\left(125 \mathrm{MHz}, \mathrm{CDCl}_{3}\right): 157.2(\mathrm{C}), 153.5(\mathrm{C}), 150.8(\mathrm{CH}), 141.8(\mathrm{C}), 138.3(\mathrm{CH})$, $138.2(\mathrm{C}), 128.7(\mathrm{CH}), 127.6(\mathrm{CH}), 126.6(\mathrm{CH}), 122.4(\mathrm{CH}), 67.0(\mathrm{CH}), 56.0\left(\mathrm{CH}_{2}\right), 18.6$ $\left(\mathrm{CH}_{3}\right), 16.9\left(\mathrm{CH}_{3}\right) ; \mathrm{m} / \mathrm{z}\left(\mathrm{ES}^{+}\right) 316[\mathrm{MH}]^{+}$; HRMS $\left(\mathrm{ES}^{+}\right)$calcd. for $\mathrm{C}_{16} \mathrm{H}_{18} \mathrm{~N}_{3} \mathrm{O}_{2} \mathrm{~S}[\mathrm{MH}]^{+}$: 316.1114, found: 316.1116 . 


\section{X-ray crystallography}

Suitable crystals were mounted on a glass fibre with Fromblin oil and placed on an Xcalibur Gemini diffractometer with a Ruby CCD area detector. The crystal was kept at 150(2) K during data collection. Using Olex $2,{ }^{7}$ the structure was solved with the ShelXT ${ }^{8}$ structure solution program using Direct Methods and refined with the $\mathrm{XL}^{9}$ refinement package using Least Squares minimisation. CCDC 1491816 crystal data: 1 d, $\mathrm{C}_{15} \mathrm{H}_{17} \mathrm{IN}_{2} \mathrm{O}_{3} \mathrm{~S} \quad(M=432.26$ g/mol): monoclinic, space group $\mathrm{P} 2{ }_{1} / \mathrm{n}$ (no. 14), $a=8.92490(6) \AA, b=12.07710(8) \AA, c=$ 15.53550(13) $\AA, \beta=98.2881(7)^{\circ}, V=1657.04(2) \AA^{3}, Z=4, T=150(2) K, \mu(\mathrm{CuK \alpha})=16.484$ $\mathrm{mm}^{-1}$, Dcalc $=1.733 \mathrm{~g} / \mathrm{cm}^{3}, 16679$ reflections measured $\left(9.312^{\circ} \leq 2 \Theta \leq 156.092^{\circ}\right), 3503$ unique $\left(R_{\text {int }}=0.0323, R_{\text {sigma }}=0.0198\right)$ which were used in all calculations. The final $R_{1}$ was $0.0302(\mathrm{I}>2 \sigma(\mathrm{I}))$ and $w R_{2}$ was 0.0816 (all data). CCDC 1491817 crystal data for $\mathbf{3 h}$ $\mathrm{C}_{18} \mathrm{H}_{17} \mathrm{ClN}_{2} \mathrm{O}_{2} \mathrm{~S} \quad(M=360.84 \mathrm{~g} / \mathrm{mol})$ : monoclinic, space group $\mathrm{P} 2_{1} / \mathrm{c}$ (no. 14), $a=$ 11.48668(7) $\AA, b=7.37203(6) \AA, c=20.25344(15) \AA, \beta=106.0333(7)^{\circ}, V=1648.35(2) \AA^{3}$, $Z=4, \quad T=150(2) \mathrm{K}, \mu($ CuKa $)=3.347 \mathrm{~mm}^{-1}, \quad$ calc $=1.454 \mathrm{~g} / \mathrm{cm}^{3}, 32278$ reflections measured $\left(8.008^{\circ} \leq 2 \Theta \leq 156.154^{\circ}\right), 3520$ unique $\left(R_{\text {int }}=0.0368, R_{\text {sigma }}=0.0191\right)$ which were used in all calculations. The final $R_{1}$ was $0.0332(\mathrm{I}>2 \sigma(\mathrm{I}))$ and $w R_{2}$ was 0.0930 (all data).

Solid state structure of $\mathbf{3 h}$ with atom labeling and thermal ellipsoids drawn at $50 \%$ probability level:

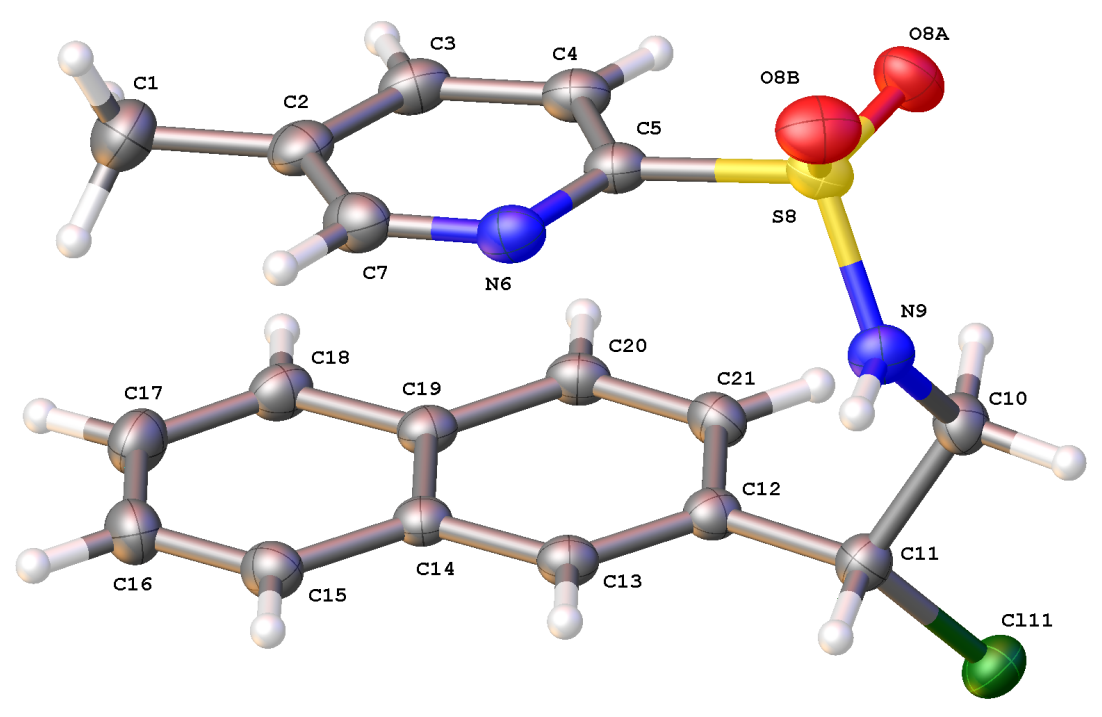




\section{9. ${ }^{1} \mathrm{H}$ and ${ }^{13} \mathrm{C}$ NMR spectra of SM1, SM2, 1a-d, 2a-I, 3a-r, and 4a-c}

\section{SM1:}

${ }^{1} \mathrm{H}\left(\mathrm{CDCl}_{3}, 500 \mathrm{MHz}\right)$<smiles>CCCOc1ccccc1I</smiles>

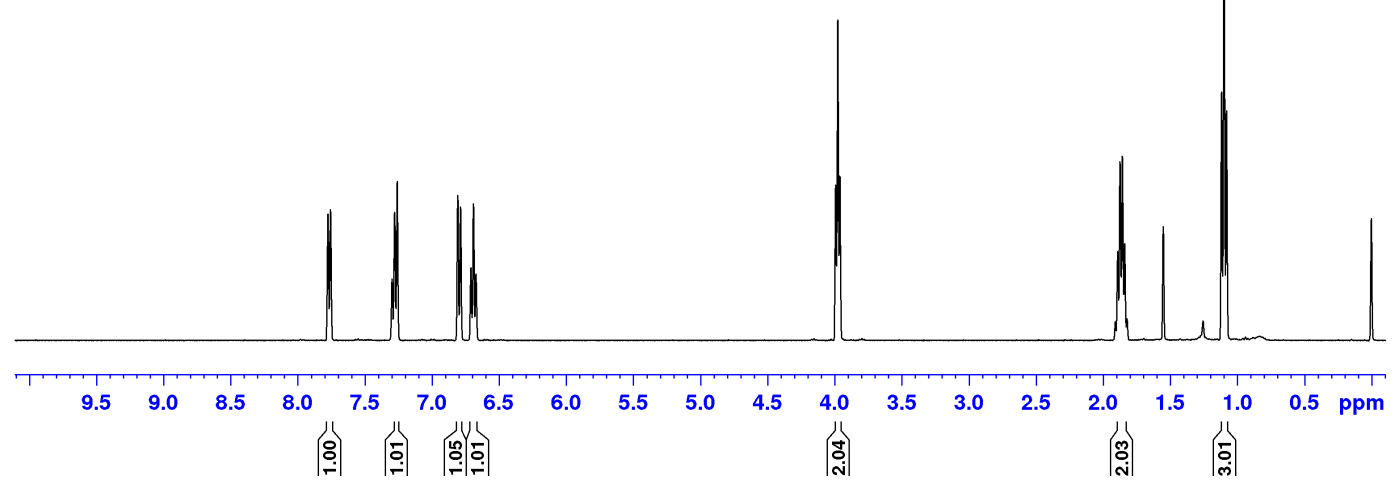

${ }^{13} \mathrm{C}\left(\mathrm{CDCl}_{3}, 125 \mathrm{MHz}\right)$

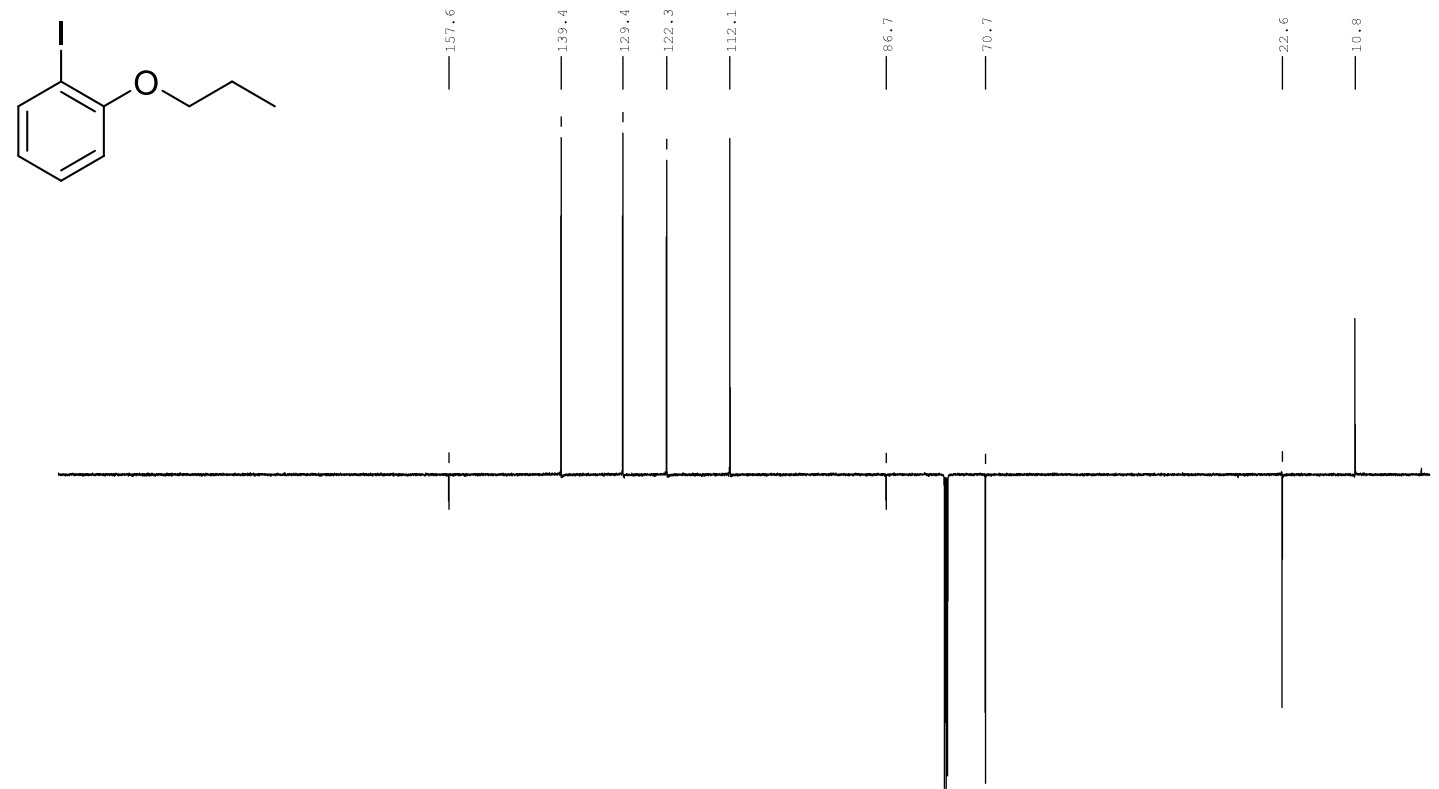


SM2:

${ }^{1} \mathrm{H}\left(\mathrm{CDCl}_{3}, 500 \mathrm{MHz}\right)$

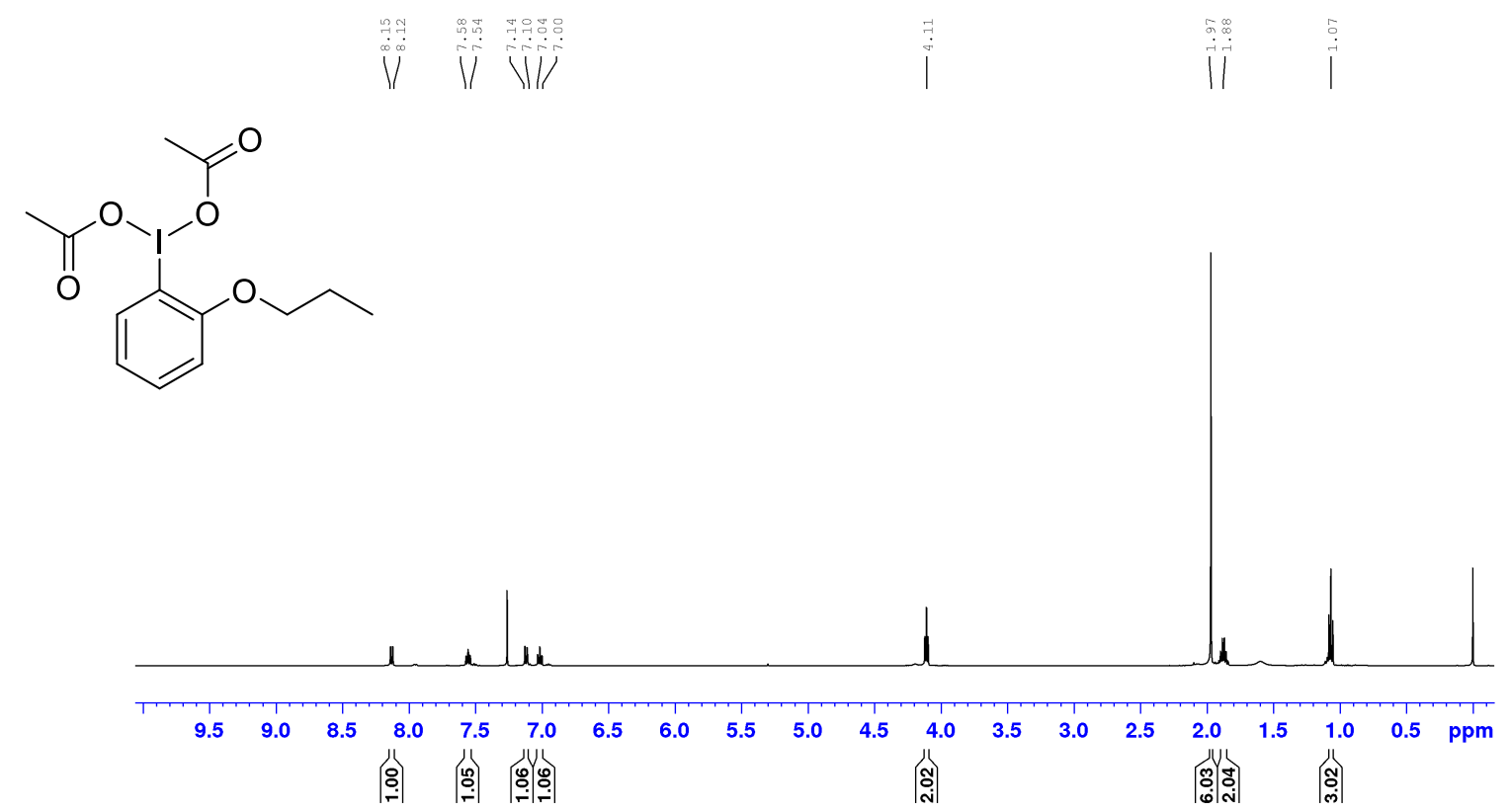

${ }^{13} \mathrm{C}\left(\mathrm{CDCl}_{3}, 125 \mathrm{MHz}\right)$<smiles>CCCOc1ccccc1I(OC(C)=O)OC(C)=O</smiles> 
1a:

${ }^{1} \mathrm{H}\left(\mathrm{CDCl}_{3}, 500 \mathrm{MHz}\right)$<smiles>CCCOc1ccccc1I=NS(=O)(=O)c1ccc(C)cc1</smiles>

1a

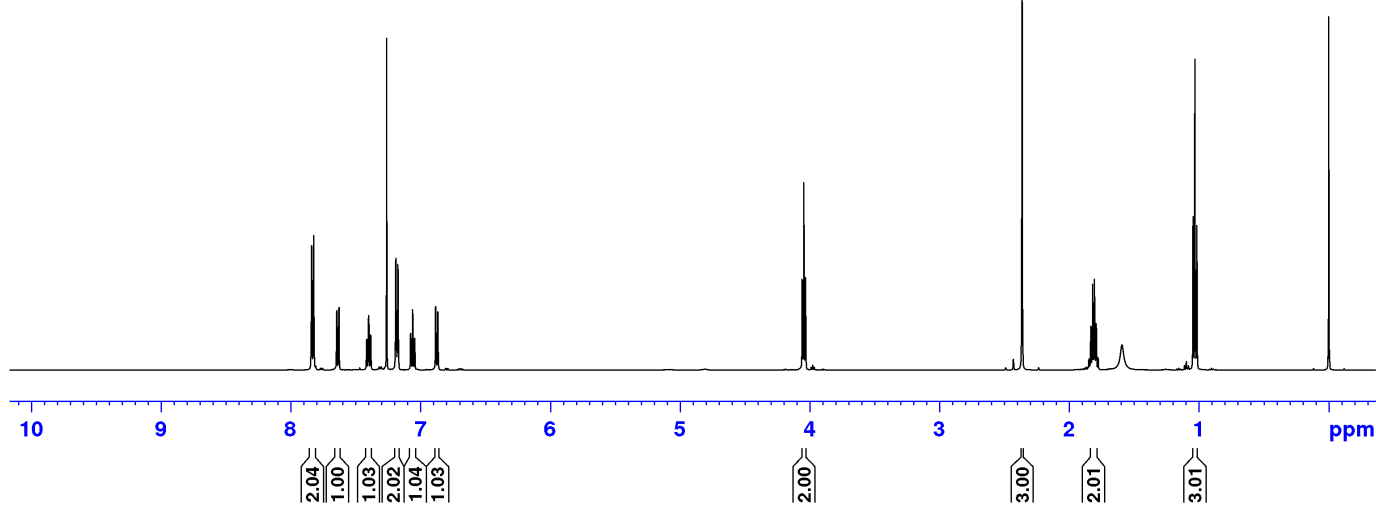

${ }^{13} \mathrm{C}\left(\mathrm{CDCl}_{3}, 125 \mathrm{MHz}\right)$

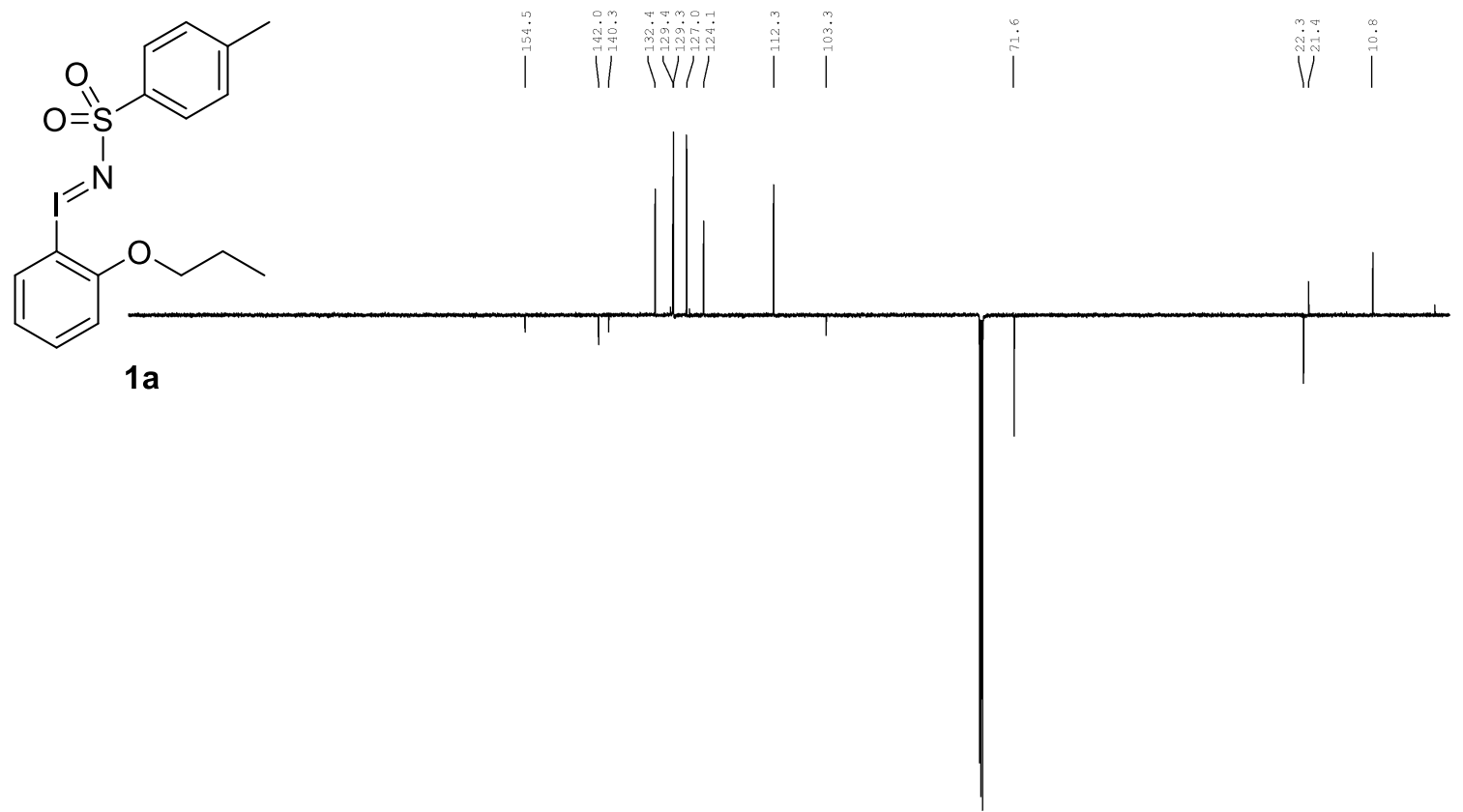

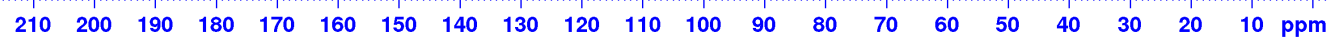


1b:

${ }^{1} \mathrm{H}\left(\mathrm{CDCl}_{3}, 500 \mathrm{MHz}\right)$<smiles>CCCOc1ccccc1I=NS(=O)(=O)c1ccc([N+](=O)[O-])cc1</smiles>

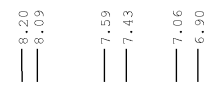

$1 b$

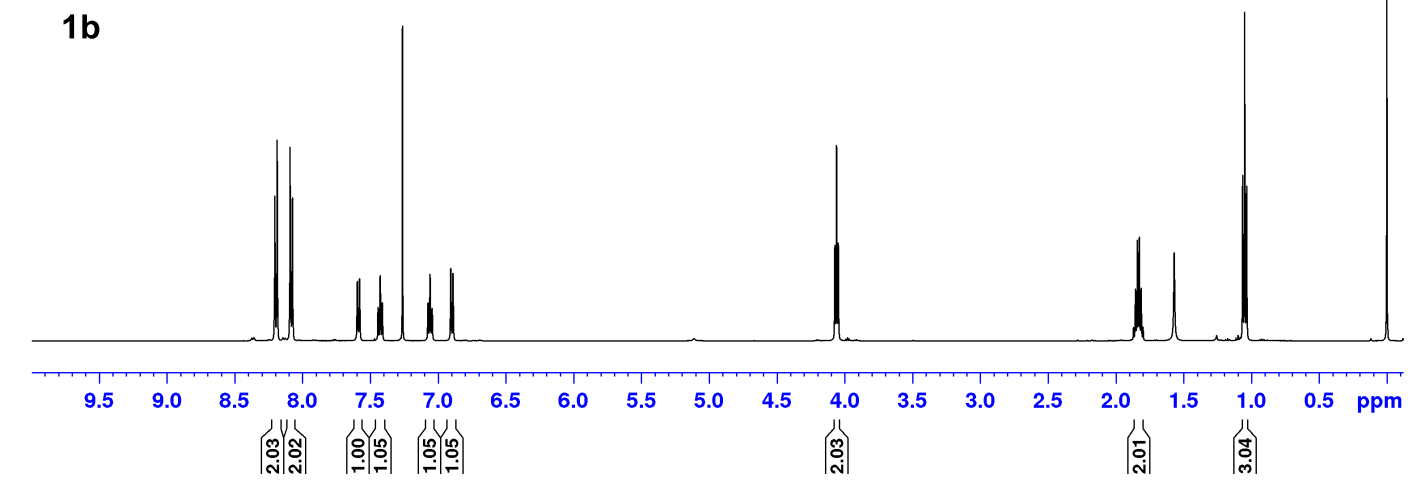

${ }^{13} \mathrm{C}\left(\mathrm{CDCl}_{3}, 125 \mathrm{MHz}\right)$<smiles>CCCOc1ccccc1I=NS(=O)(=O)c1ccc([N+](=O)[O-])cc1</smiles>

1b

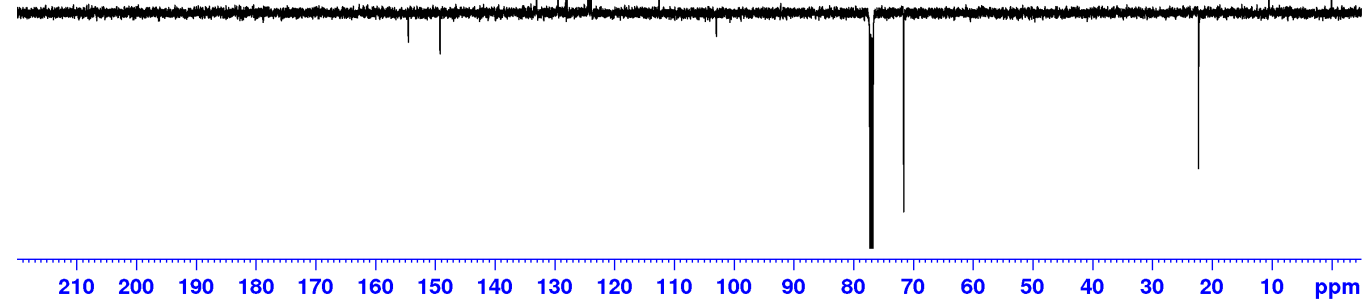


1c:

${ }^{1} \mathrm{H}\left(\mathrm{CDCl}_{3}, 500 \mathrm{MHz}\right)$
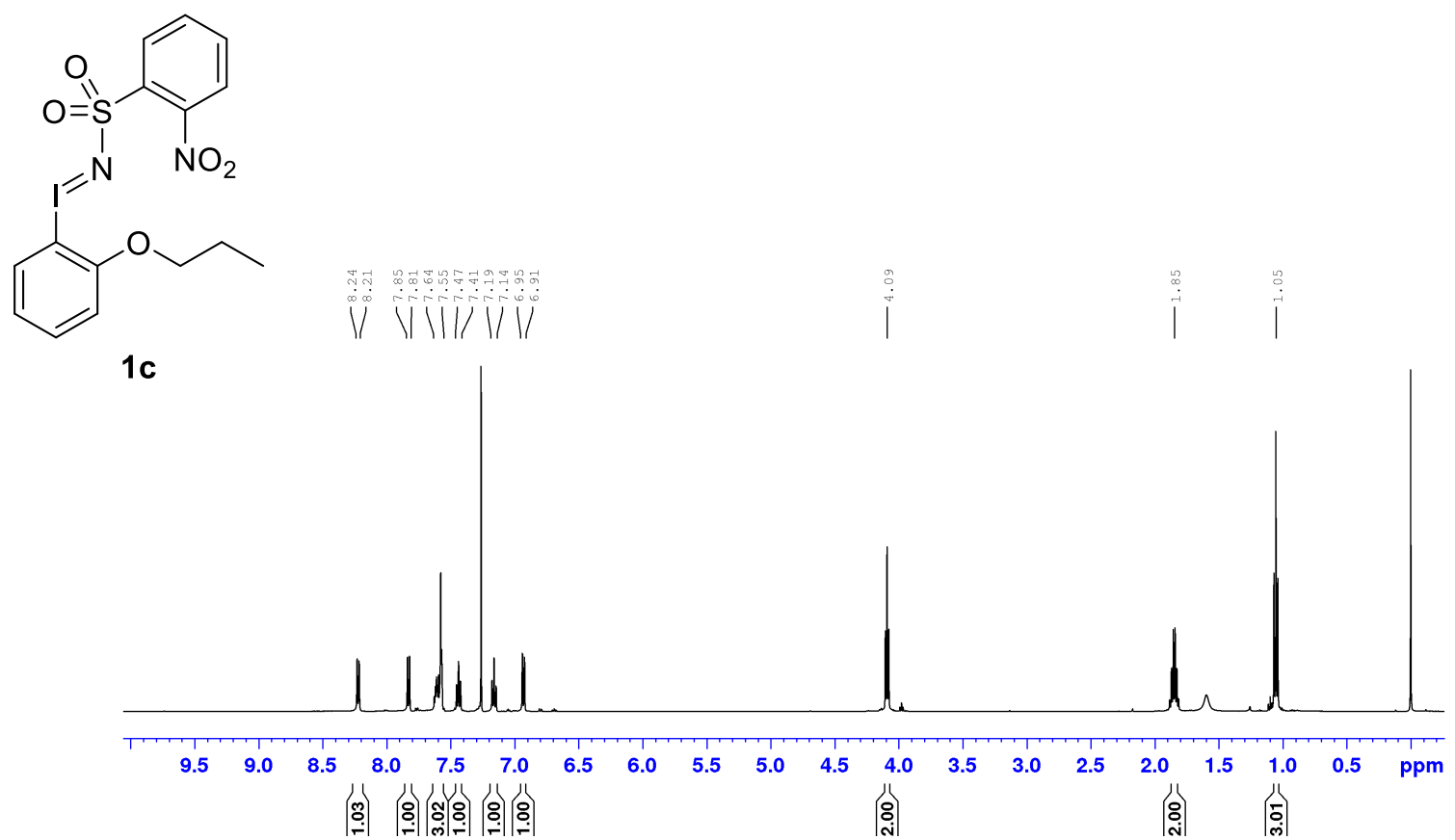

${ }^{13} \mathrm{C}\left(\mathrm{CDCl}_{3}, 125 \mathrm{MHz}\right)$<smiles>CCCOc1ccccc1/N=N/S(=O)(=O)c1ccccc1[N+](=O)[O-]</smiles>

$1 c$
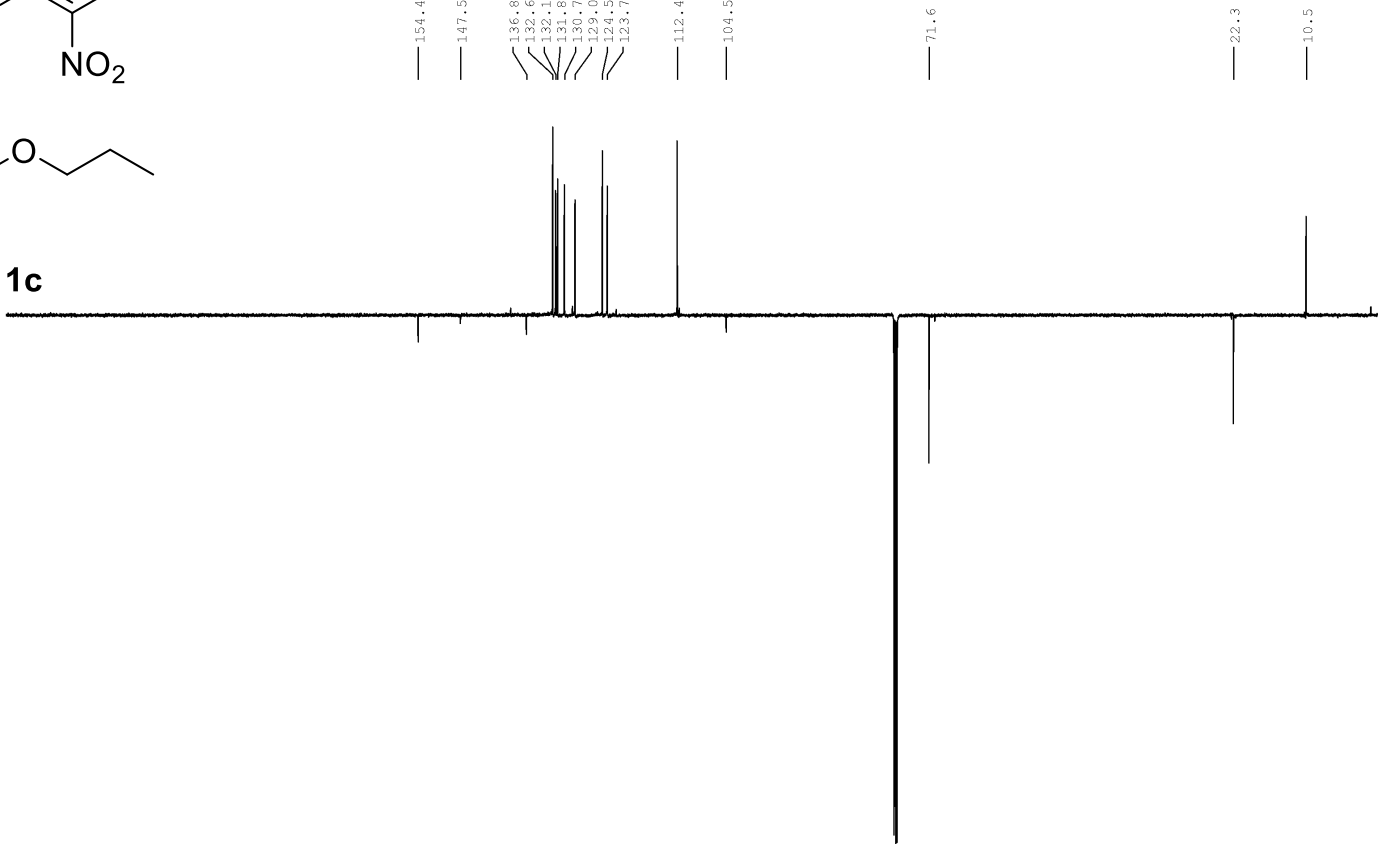

$\begin{array}{lllllllllllllllllllll}210 & 200 & 190 & 180 & 170 & 160 & 150 & 140 & 130 & 120 & 110 & 100 & 90 & 80 & 70 & 60 & 50 & 40 & 30 & 20 & \mathrm{ppm}\end{array}$ 
1d:

${ }^{1} \mathrm{H}\left(\mathrm{CDCl}_{3}, 500 \mathrm{MHz}\right)$

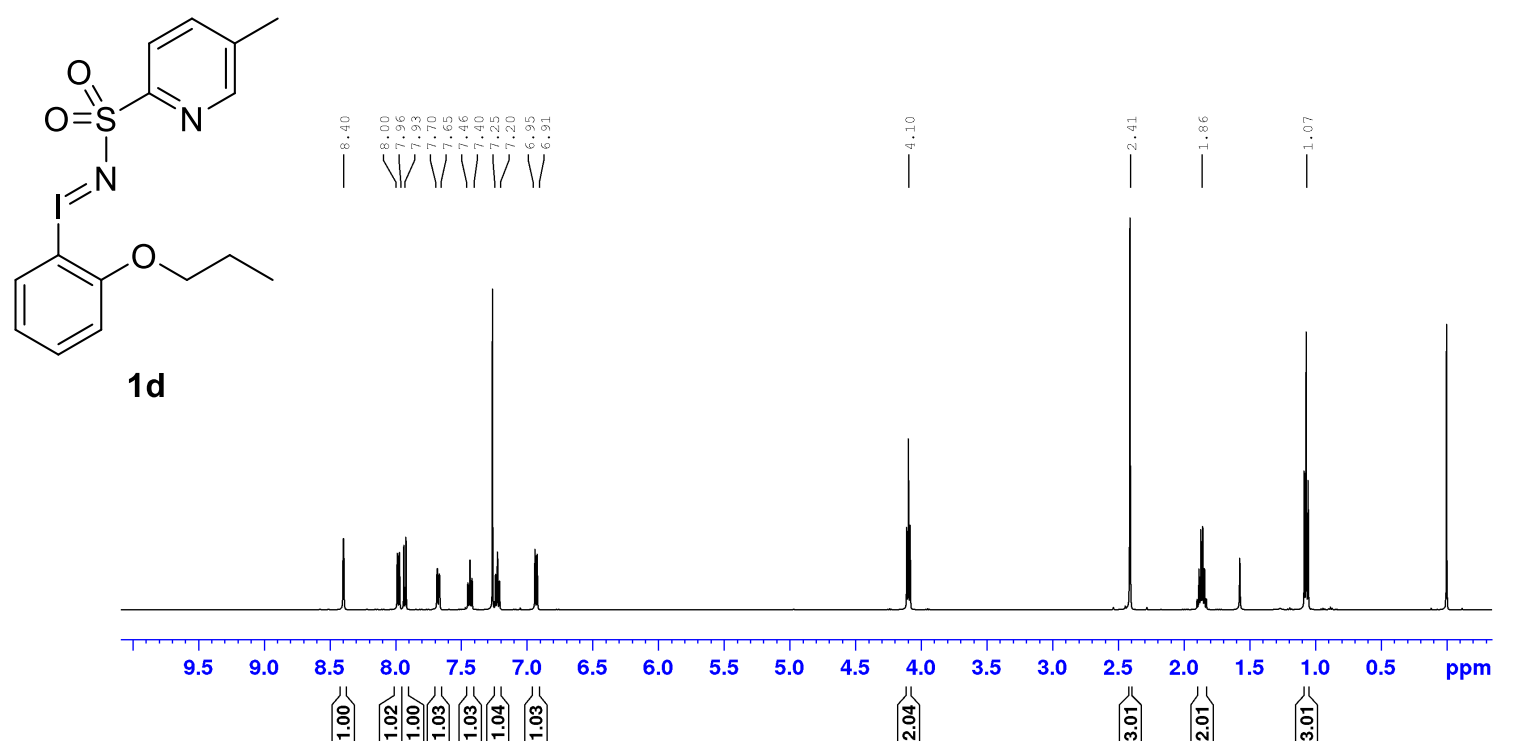

${ }^{13} \mathrm{C}\left(\mathrm{CDCl}_{3}, 125 \mathrm{MHz}\right)$

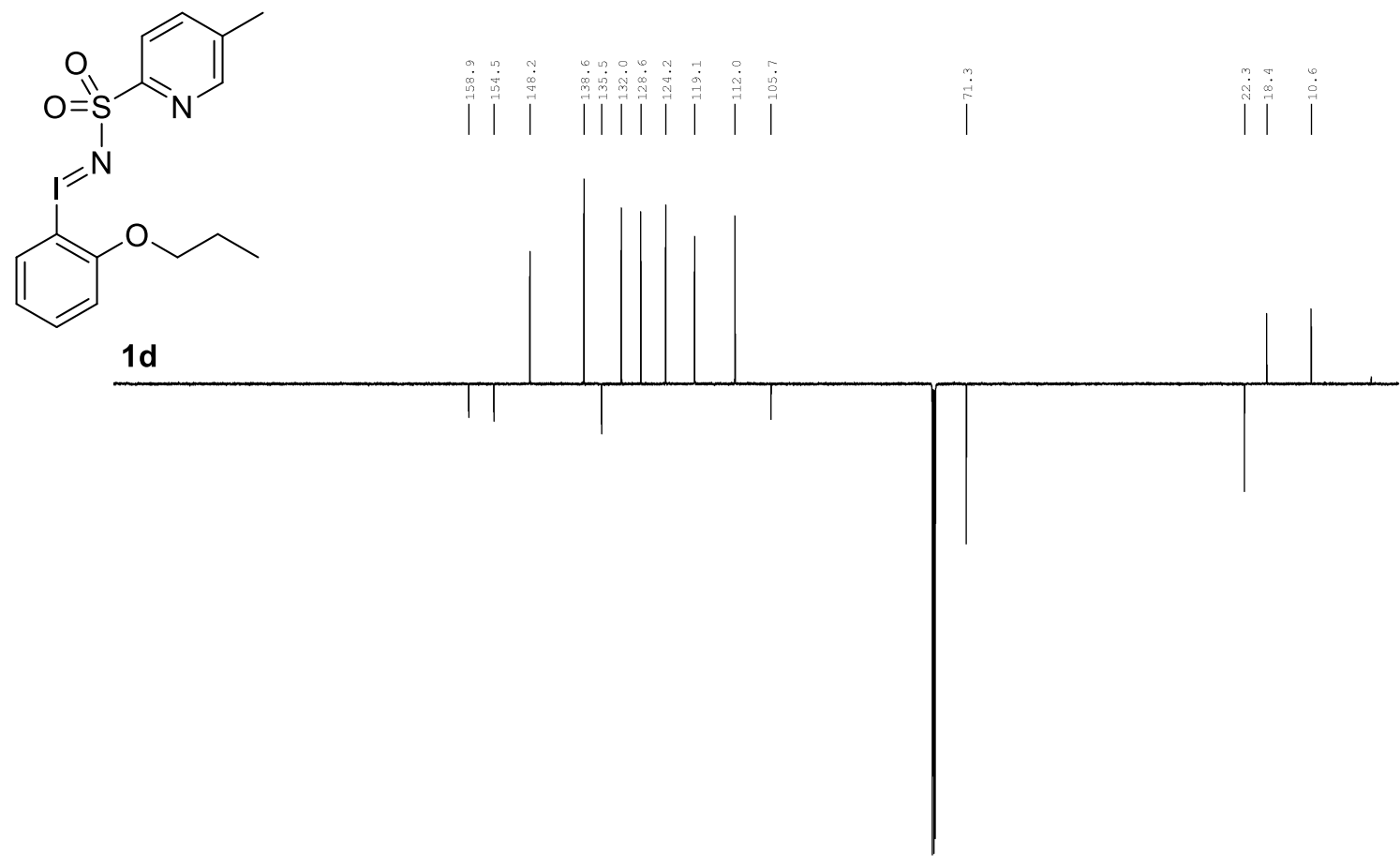

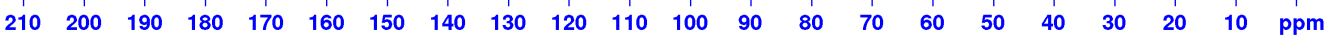


2a:

${ }^{1} \mathrm{H}\left(\mathrm{CDCl}_{3}, 500 \mathrm{MHz}\right)$
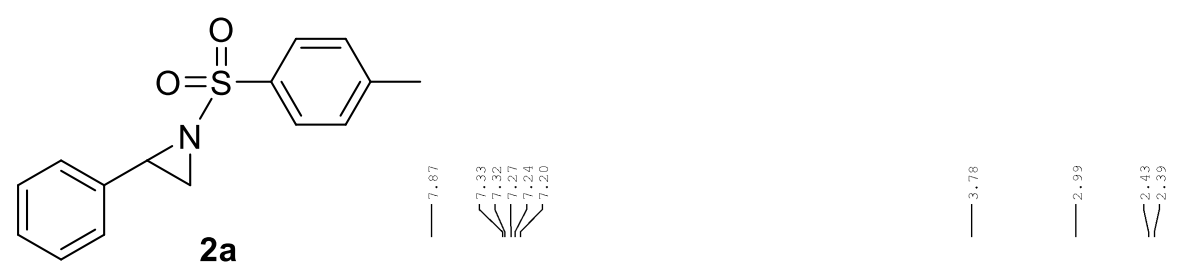

${ }^{13} \mathrm{C}\left(\mathrm{CDCl}_{3}, 125 \mathrm{MHz}\right)$

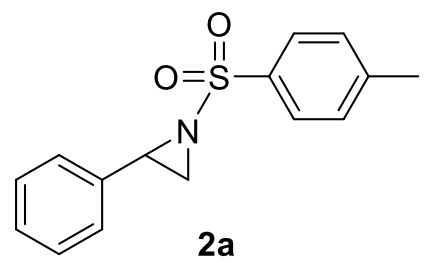

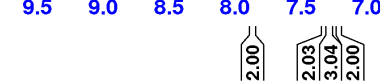
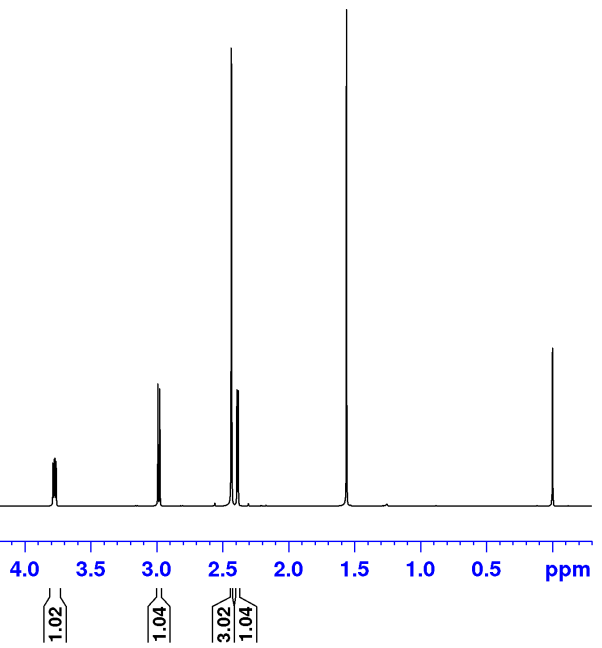

$\begin{array}{lllllllllllllllllllll}210 & 200 & 190 & 180 & 170 & 160 & 150 & 140 & 130 & 120 & 110 & 100 & 90 & 80 & 70 & 60 & 50 & 40 & 30 & 20 & 10\end{array}$ 
2b:

${ }^{1} \mathrm{H}\left(\mathrm{CDCl}_{3}, 500 \mathrm{MHz}\right)$

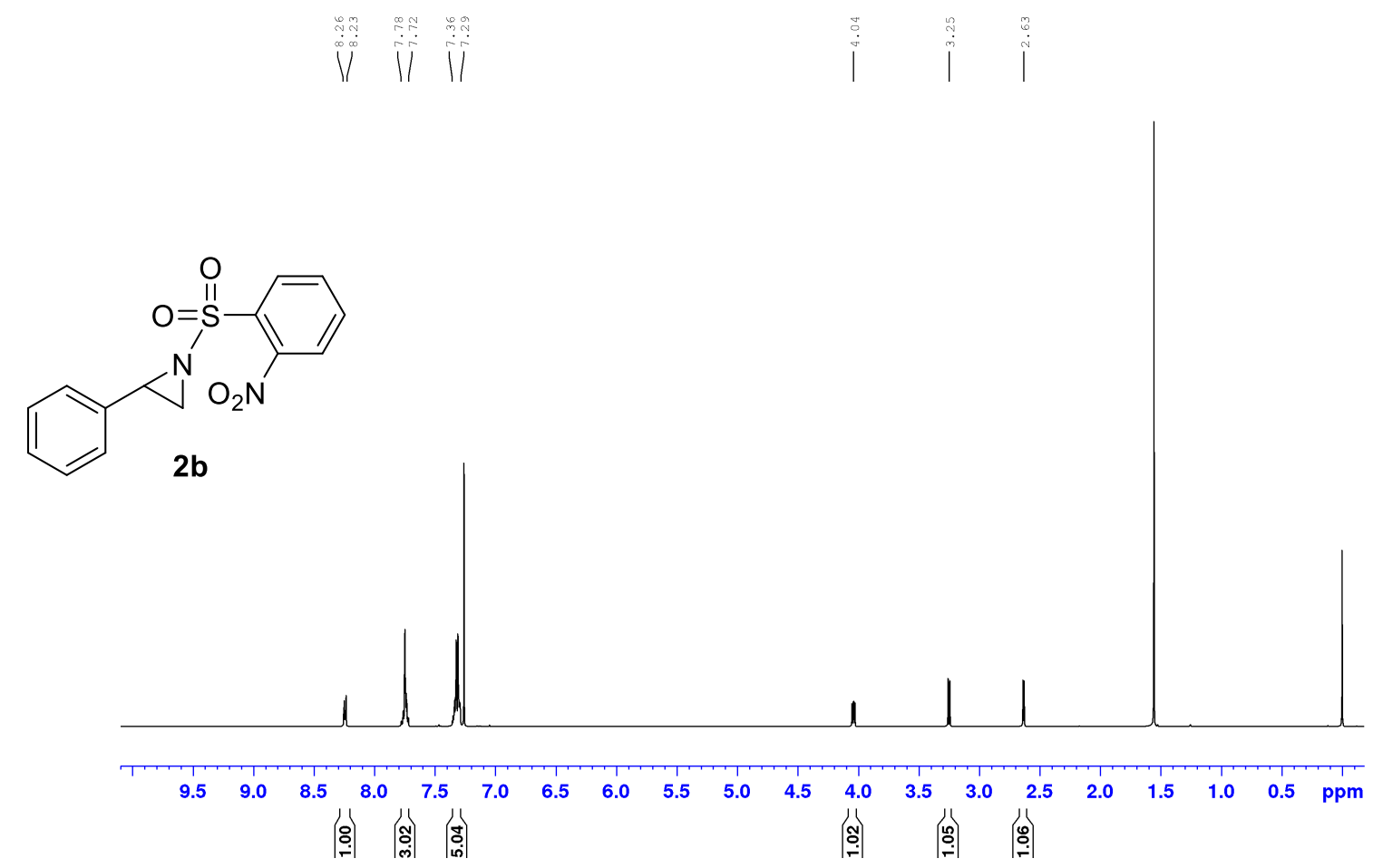

${ }^{13} \mathrm{C}\left(\mathrm{CDCl}_{3}, 125 \mathrm{MHz}\right)$

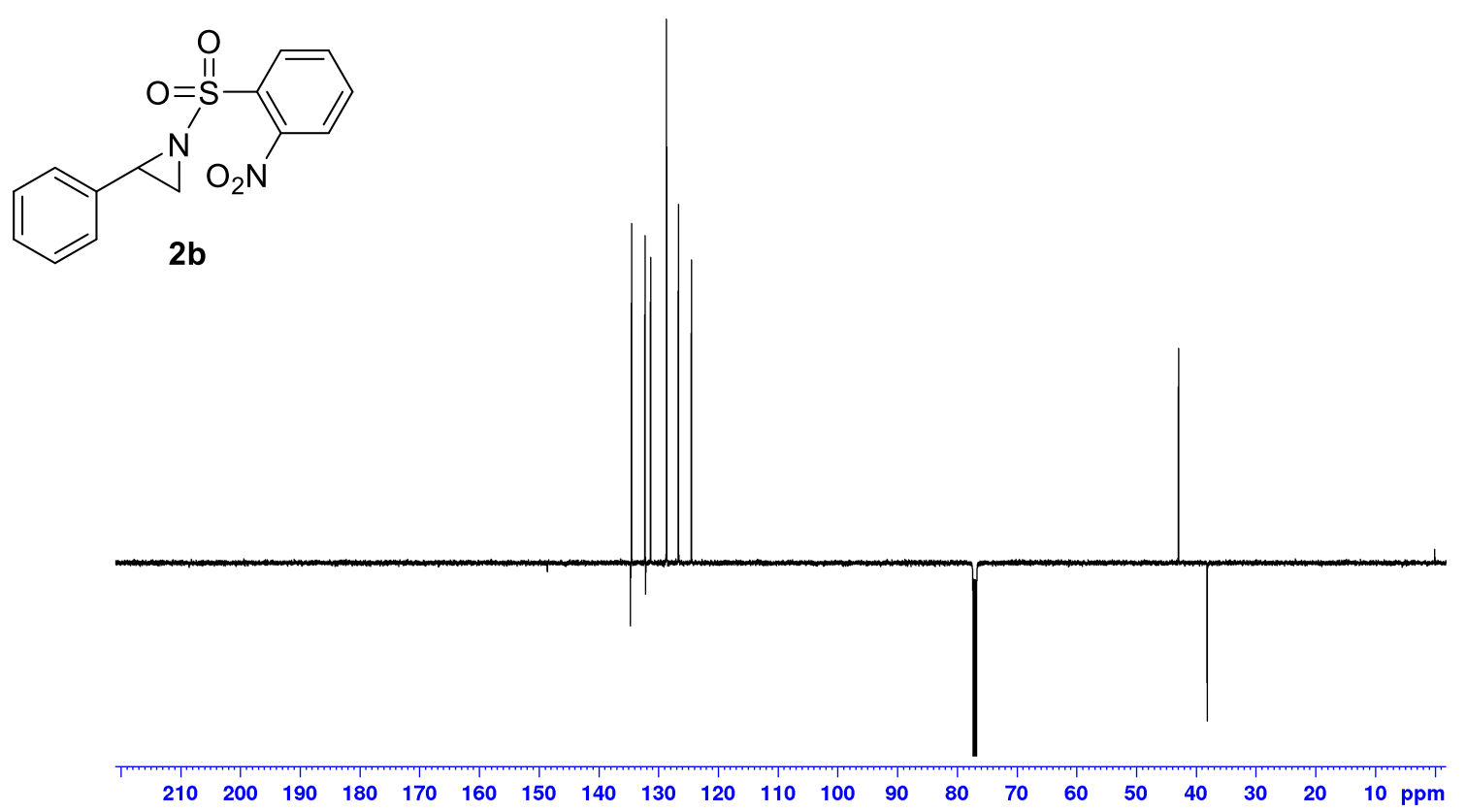


2c:

${ }^{1} \mathrm{H}\left(\mathrm{CDCl}_{3}, 500 \mathrm{MHz}\right)$

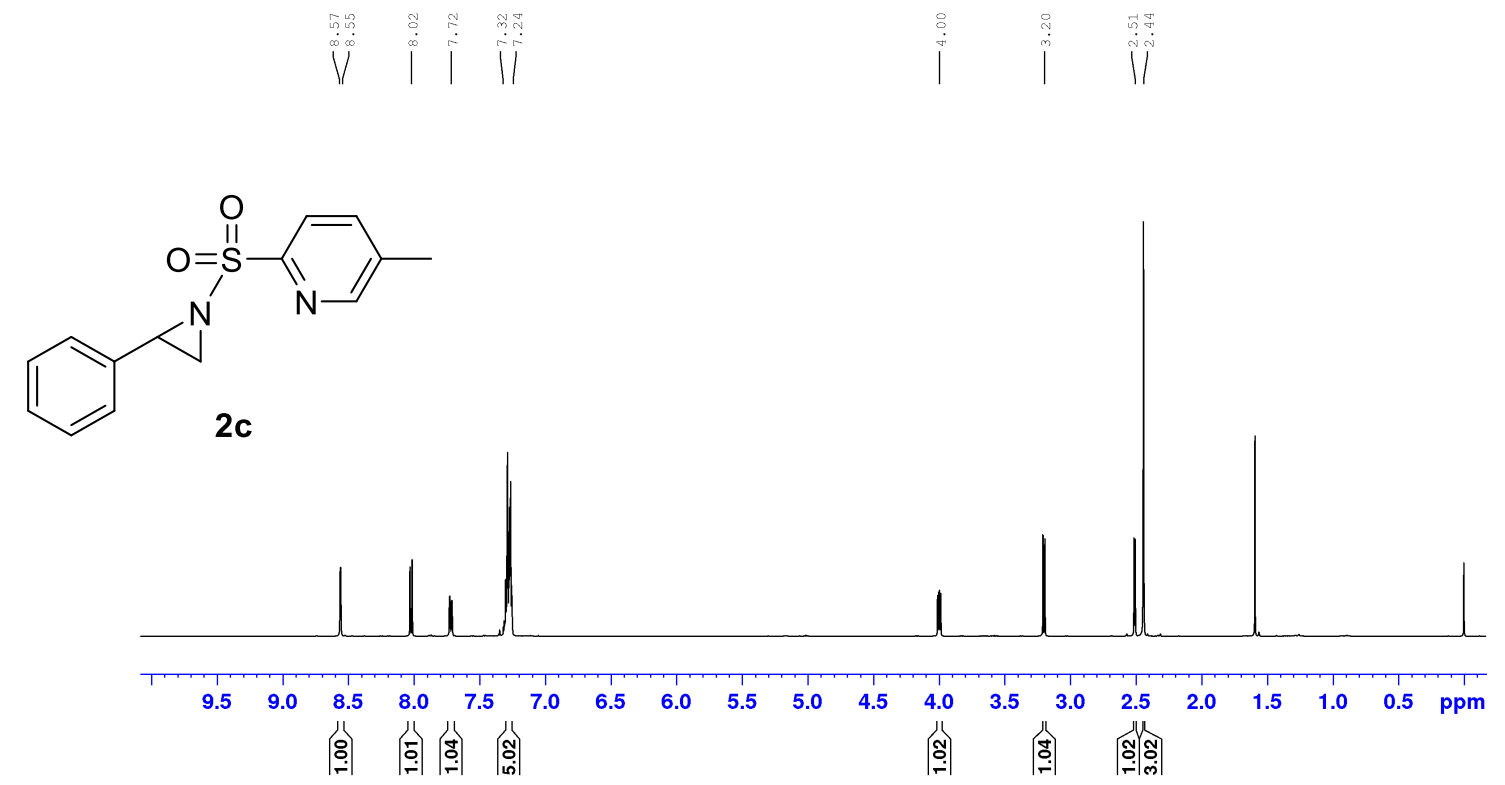

${ }^{13} \mathrm{C}\left(\mathrm{CDCl}_{3}, 125 \mathrm{MHz}\right)$<smiles>Cc1ccc(S(=O)(=O)N2CC2c2ccccc2)nc1</smiles> 
2d:

${ }^{1} \mathrm{H}\left(\mathrm{CDCl}_{3}, 500 \mathrm{MHz}\right)$

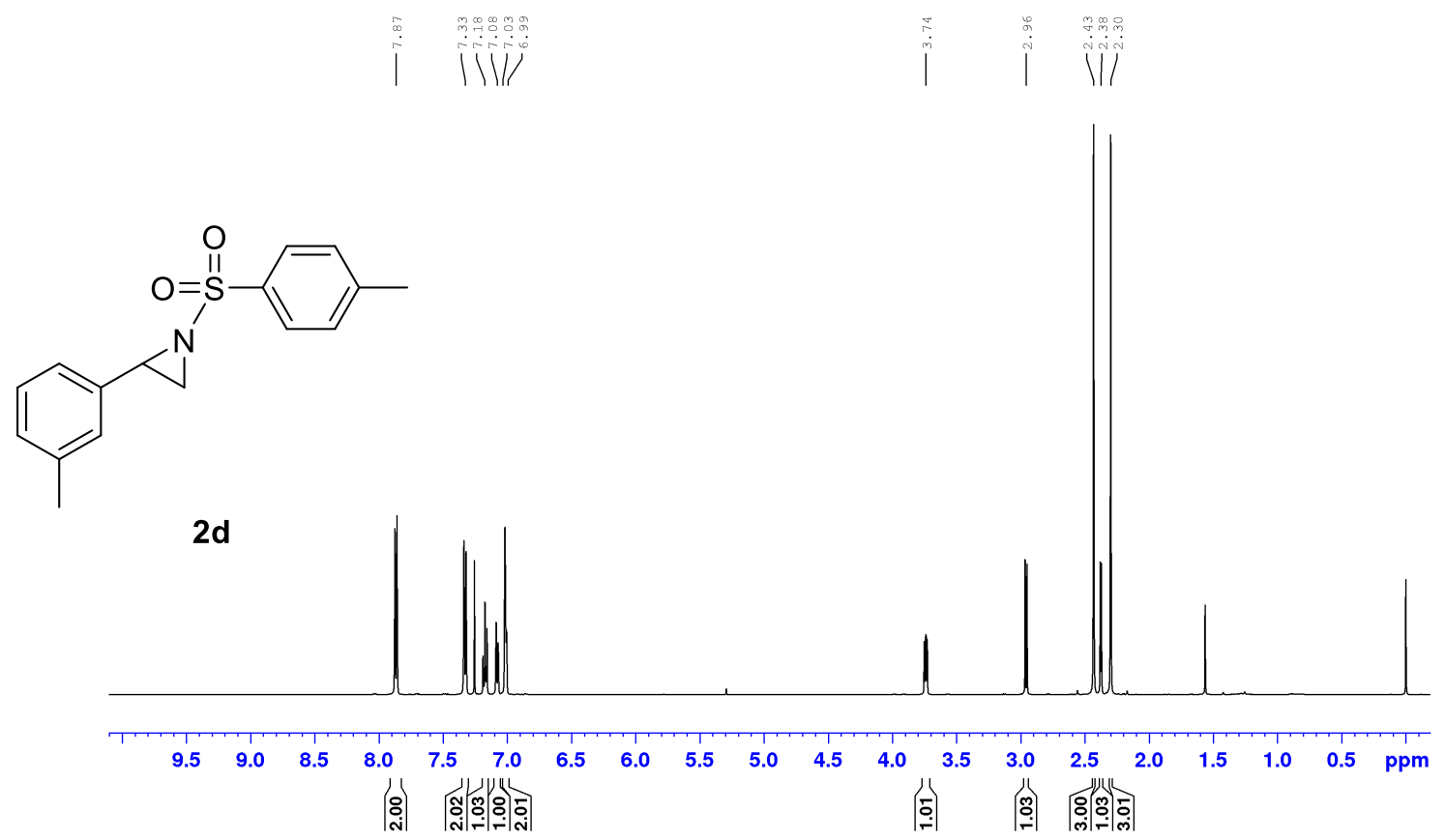

${ }^{13} \mathrm{C}\left(\mathrm{CDCl}_{3}, 125 \mathrm{MHz}\right)$
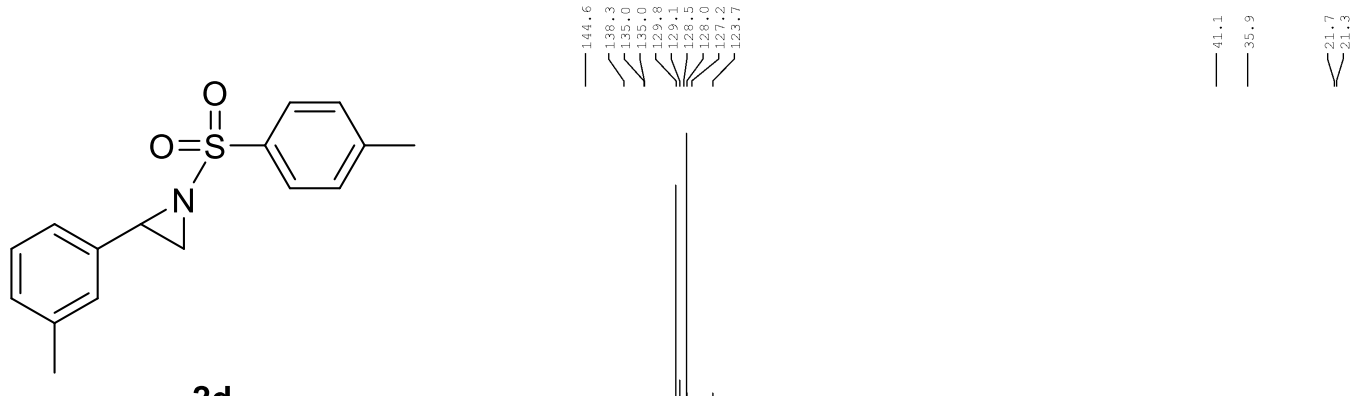

2d

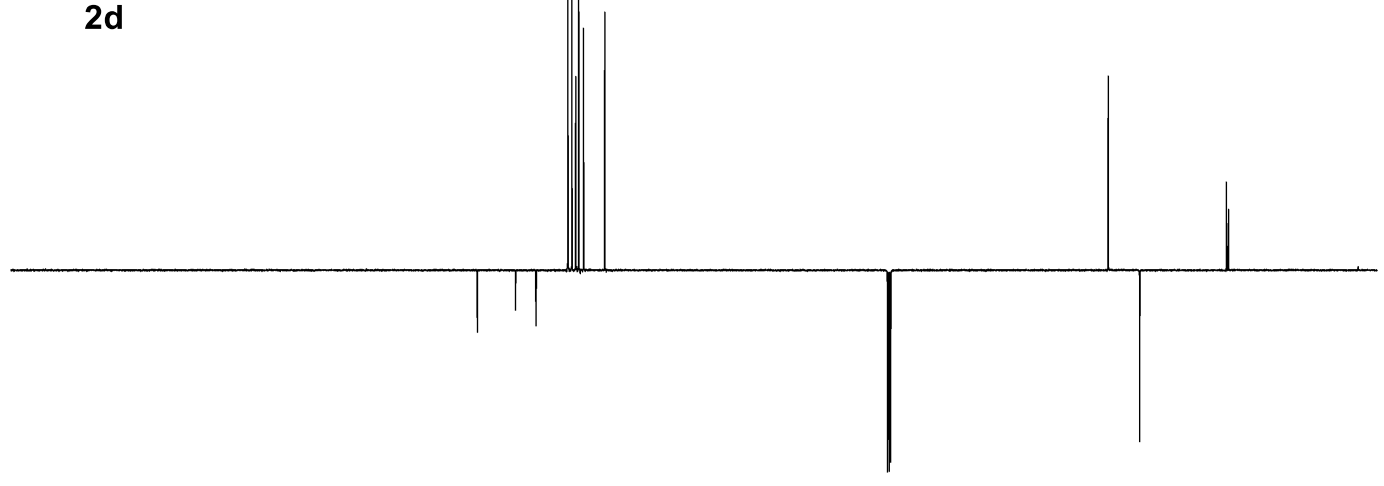

$\begin{array}{llllllllllllllllllllll}210 & 200 & 190 & 180 & 170 & 160 & 150 & 140 & 130 & 120 & 110 & 100 & 90 & 80 & 70 & 60 & 50 & 40 & 30 & 20 & 10 & \mathrm{ppm}\end{array}$ 
2e:

${ }^{1} \mathrm{H}\left(\mathrm{CDCl}_{3}, 500 \mathrm{MHz}\right)$

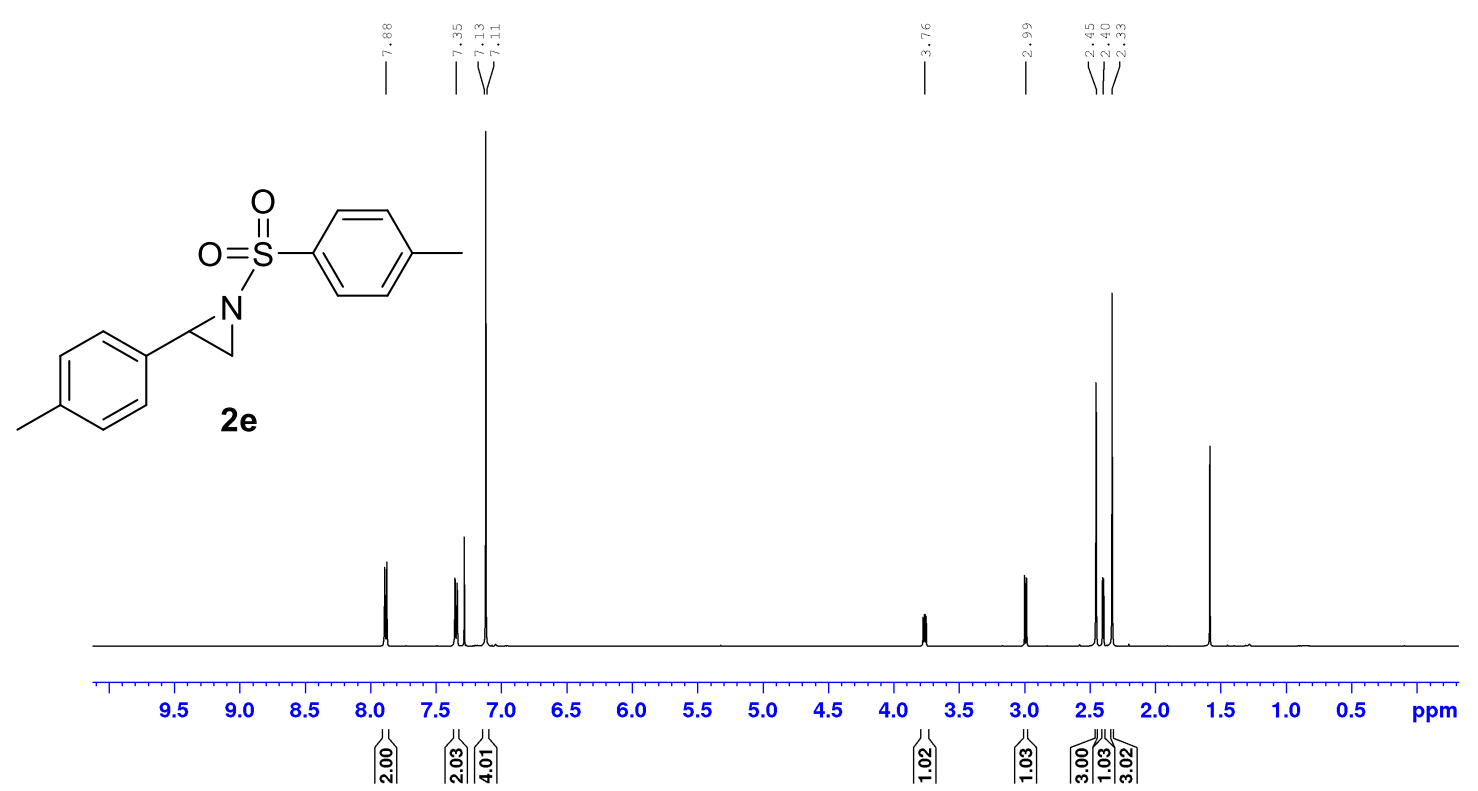

${ }^{13} \mathrm{C}\left(\mathrm{CDCl}_{3}, 125 \mathrm{MHz}\right)$
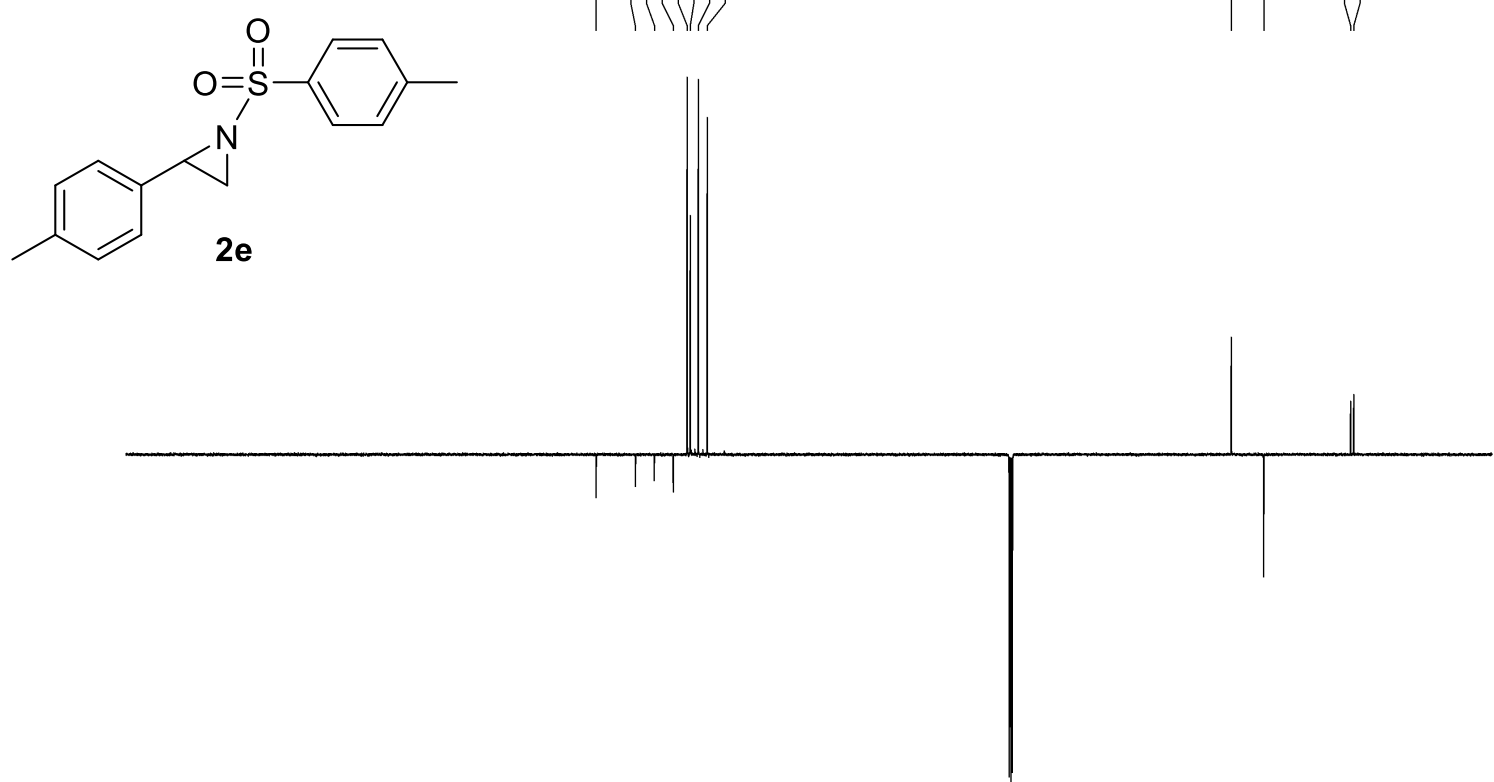

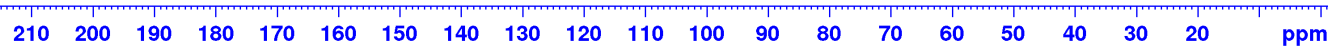


2f:

${ }^{1} \mathrm{H}\left(\mathrm{CD}_{2} \mathrm{Cl}_{2}, 500 \mathrm{MHz}\right)$

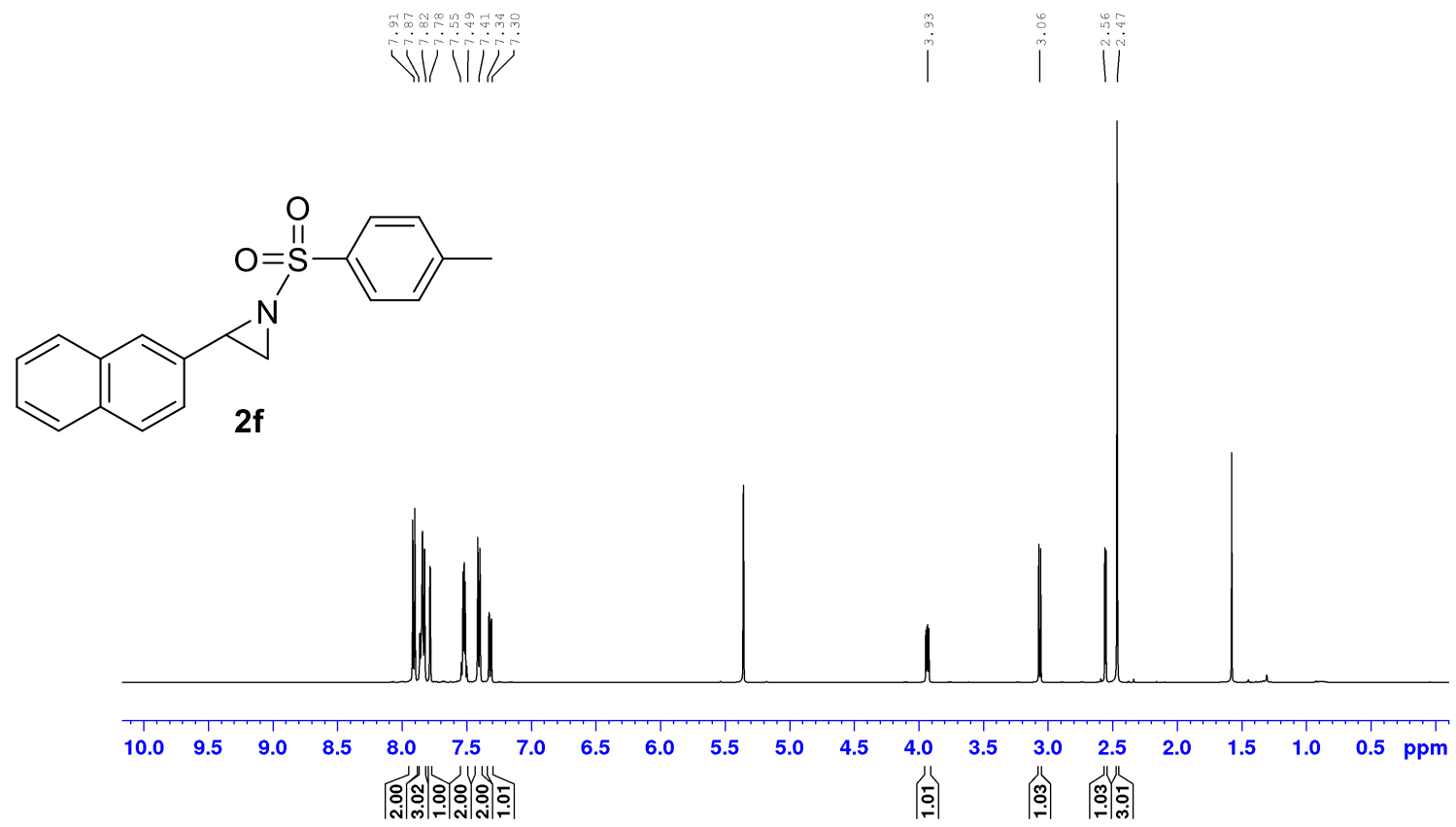

${ }^{13} \mathrm{C}\left(\mathrm{CD}_{2} \mathrm{Cl}_{2}, 125 \mathrm{MHz}\right)$
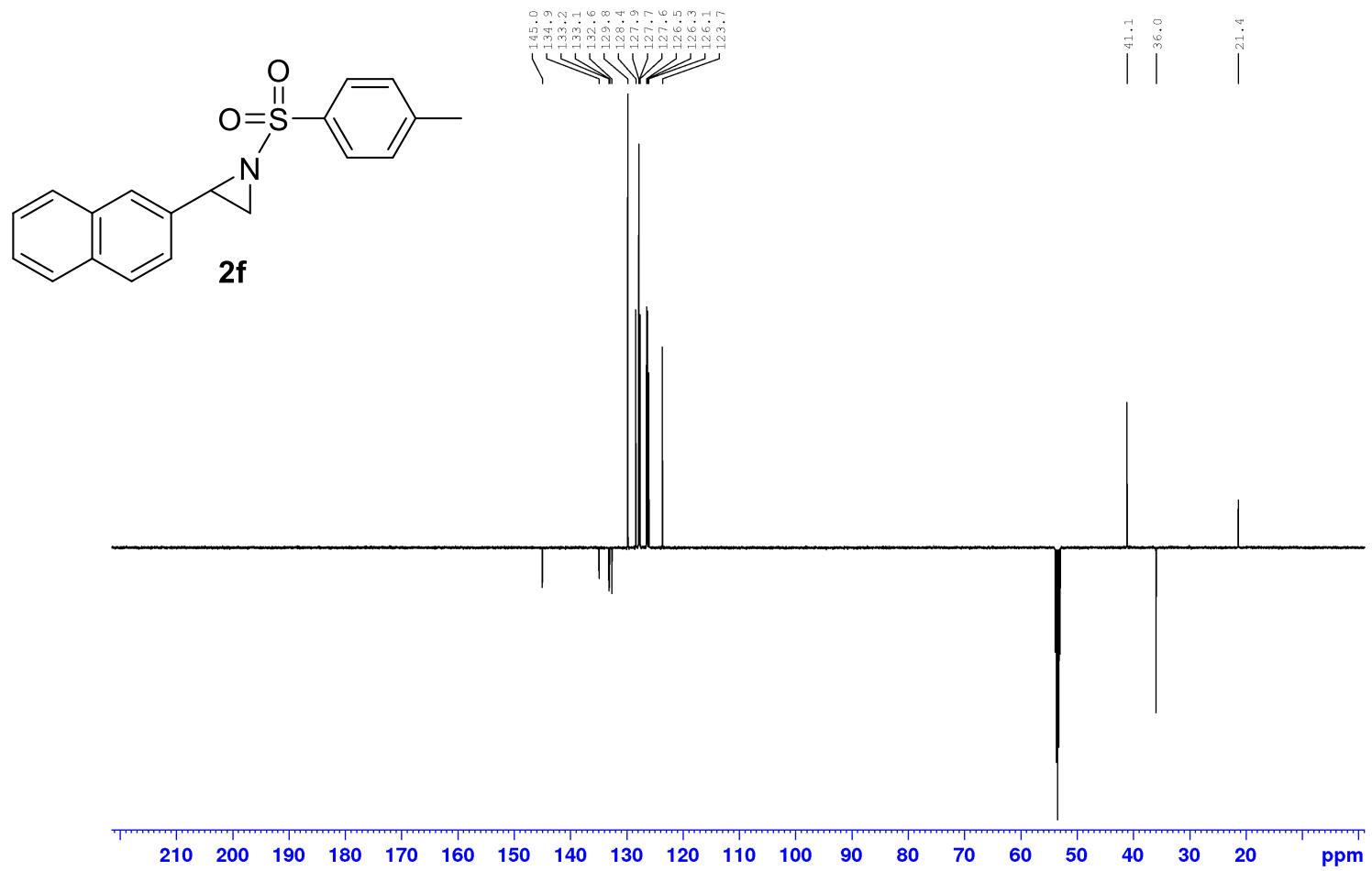
2g:

${ }^{1} \mathrm{H}\left(\mathrm{CDCl}_{3}, 500 \mathrm{MHz}\right)$<smiles>O=[N+]([O-])c1ccccc1S(=O)(=O)N1C2C3CCC(C3)C21</smiles>

V

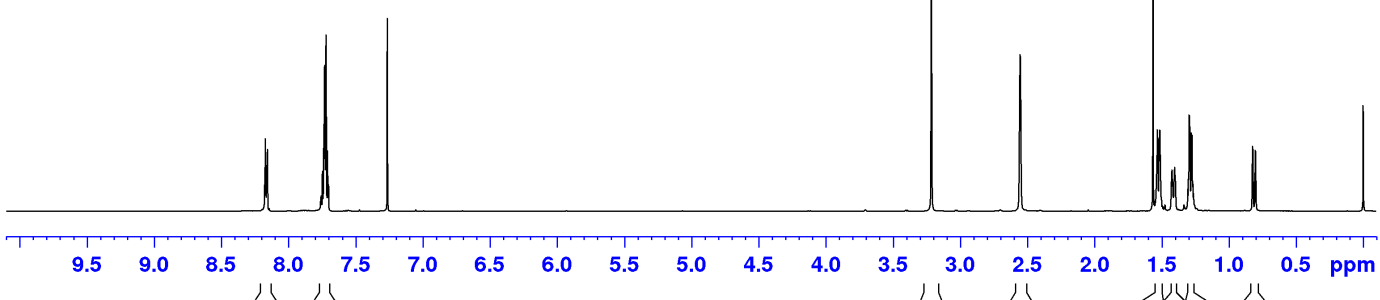

|

(大)

${ }^{13} \mathrm{C}\left(\mathrm{CDCl}_{3}, 125 \mathrm{MHz}\right)$<smiles>O=[N+]([O-])c1ccccc1S(=O)(=O)N1C2C3CCC(C3)C21</smiles>

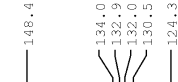

$2 \mathrm{~g}$

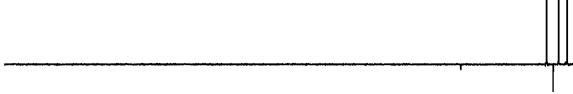


2h:

${ }^{1} \mathrm{H}\left(\mathrm{CDCl}_{3}, 500 \mathrm{MHz}\right)$

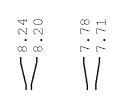<smiles>O=[N+]([O-])c1ccccc1S(=O)(=O)N1C2CCCCCC21</smiles>

$2 \mathrm{~h}$
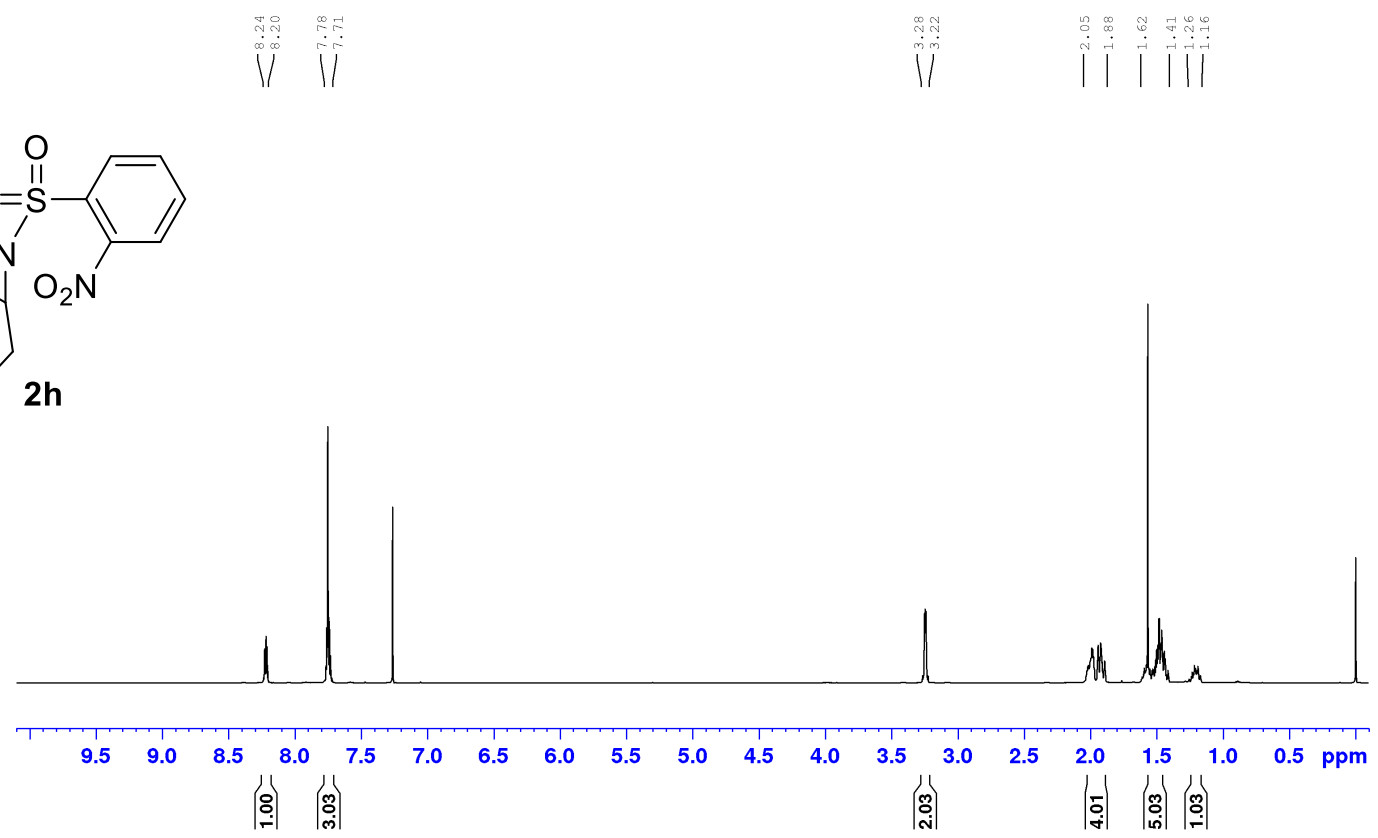

${ }^{13} \mathrm{C}\left(\mathrm{CDCl}_{3}, 125 \mathrm{MHz}\right)$

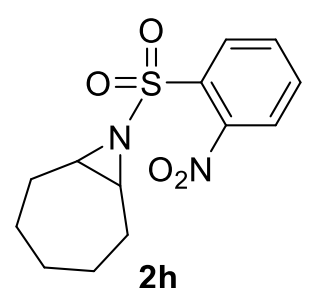

$2 \mathrm{~h}$
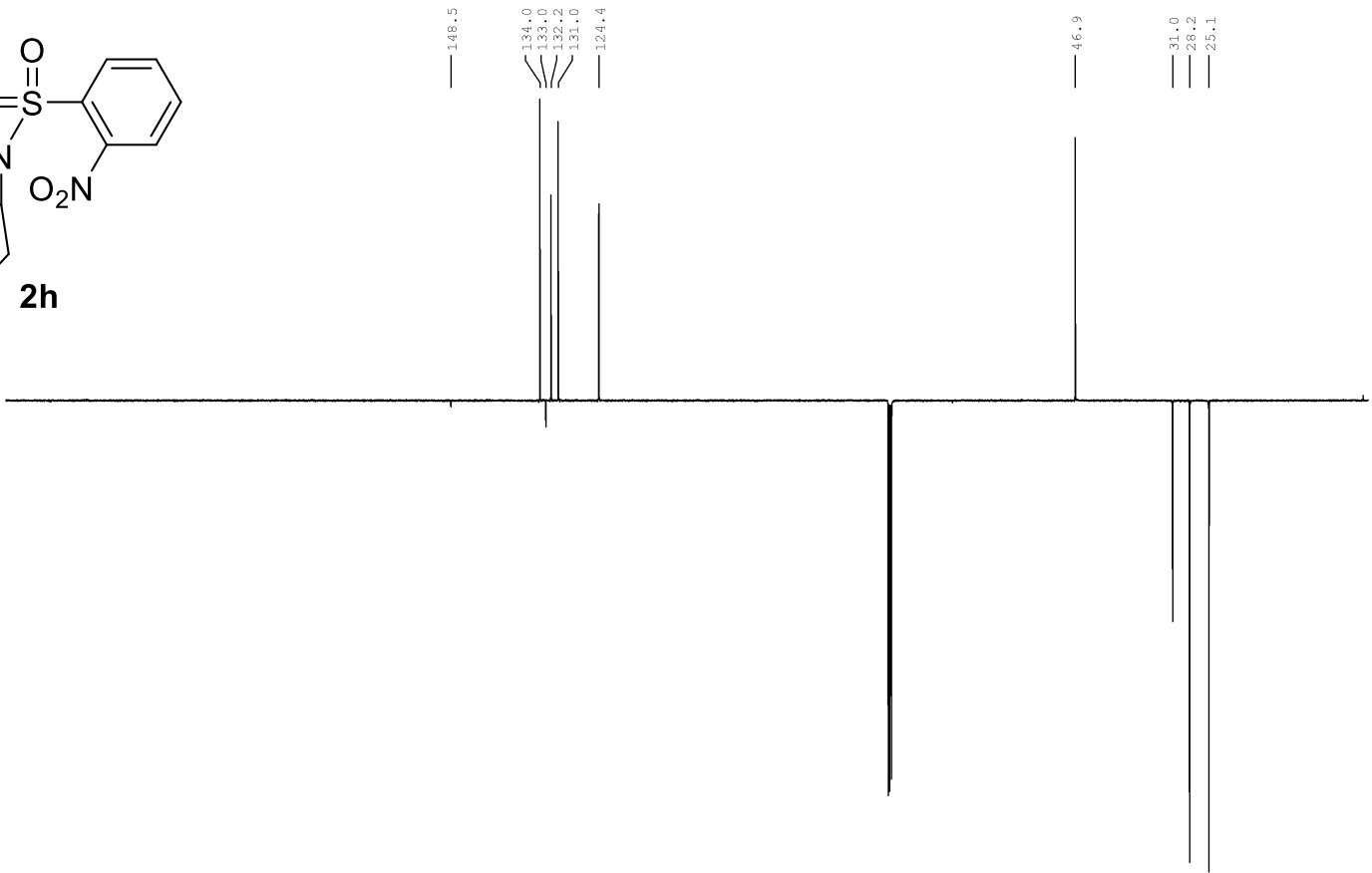

$\begin{array}{lllllllllllllllllllll}210 & 200 & 190 & 180 & 170 & 160 & 150 & 140 & 130 & 120 & 110 & 100 & 90 & 80 & 70 & 60 & 50 & 40 & 30 & 20 & \mathrm{ppm}\end{array}$ 
2i:

${ }^{1} \mathrm{H}\left(\mathrm{CDCl}_{3}, 500 \mathrm{MHz}\right)$

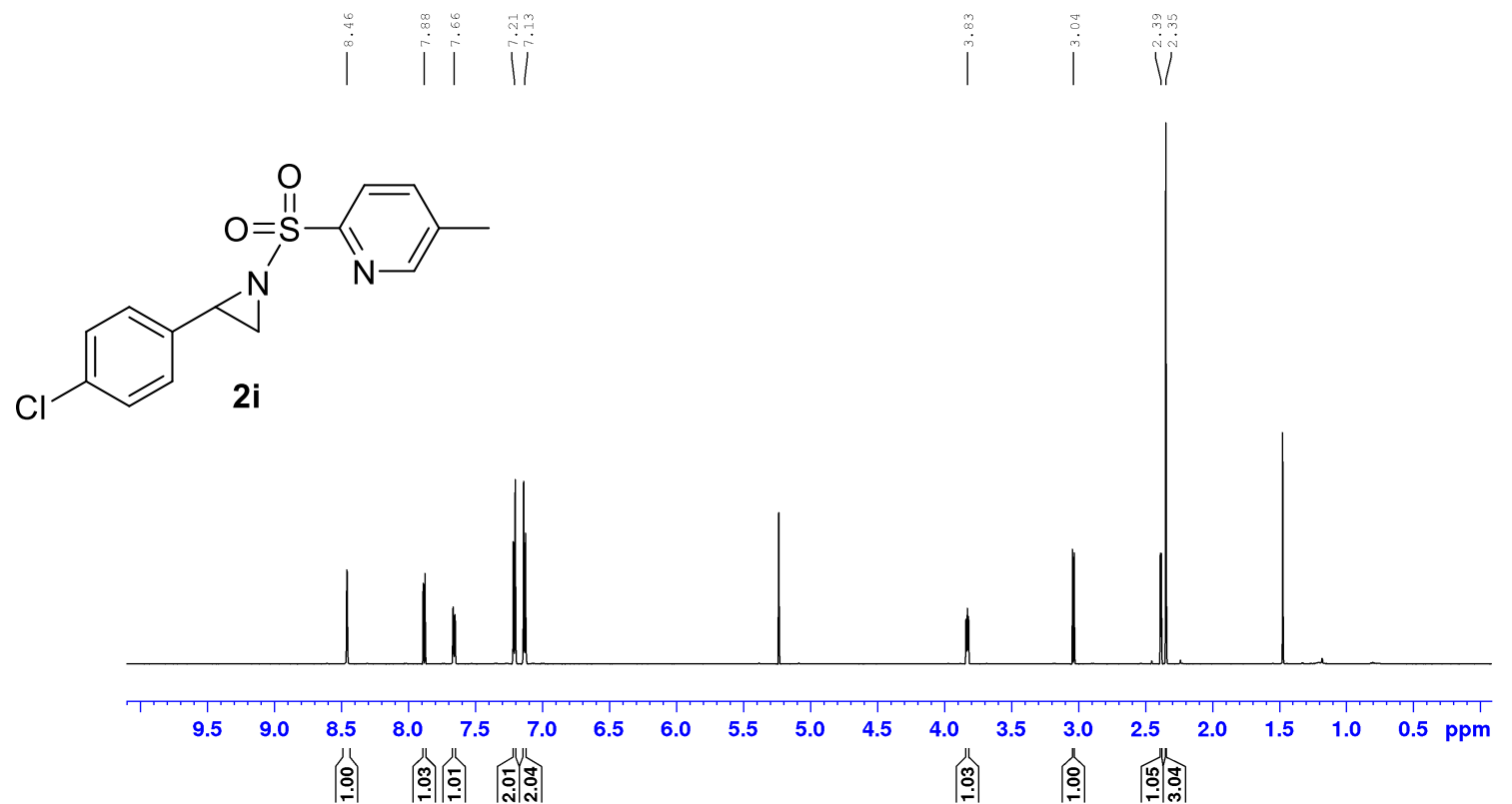

${ }^{13} \mathrm{C}\left(\mathrm{CDCl}_{3}, 125 \mathrm{MHz}\right)$

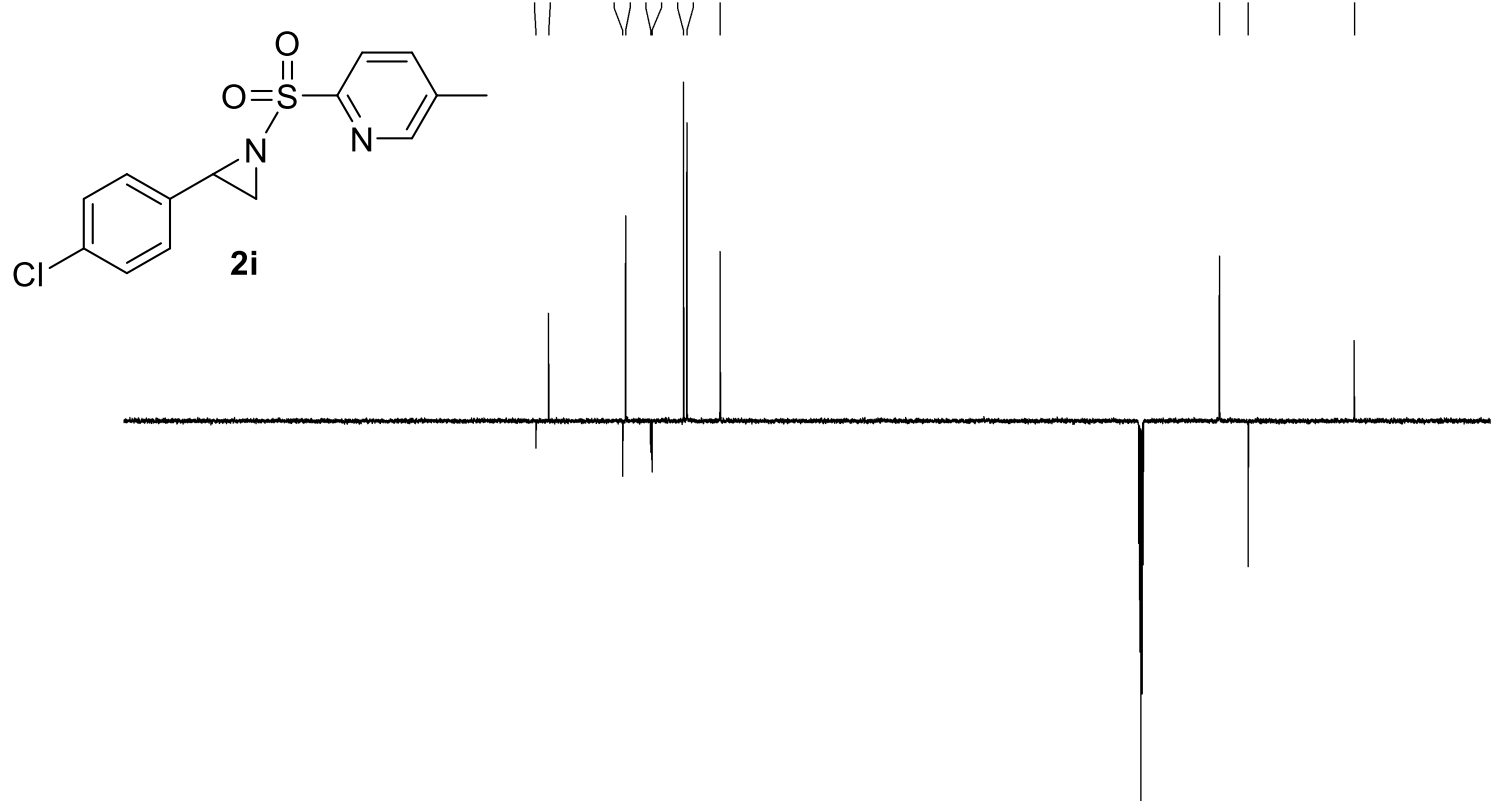

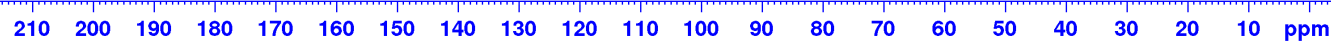


2j:

${ }^{1} \mathrm{H}\left(\mathrm{CDCl}_{3}, 500 \mathrm{MHz}\right)$

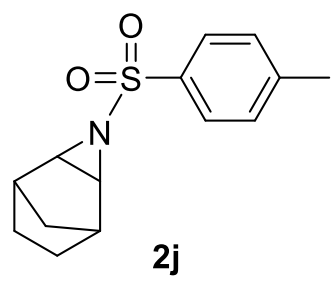

V V

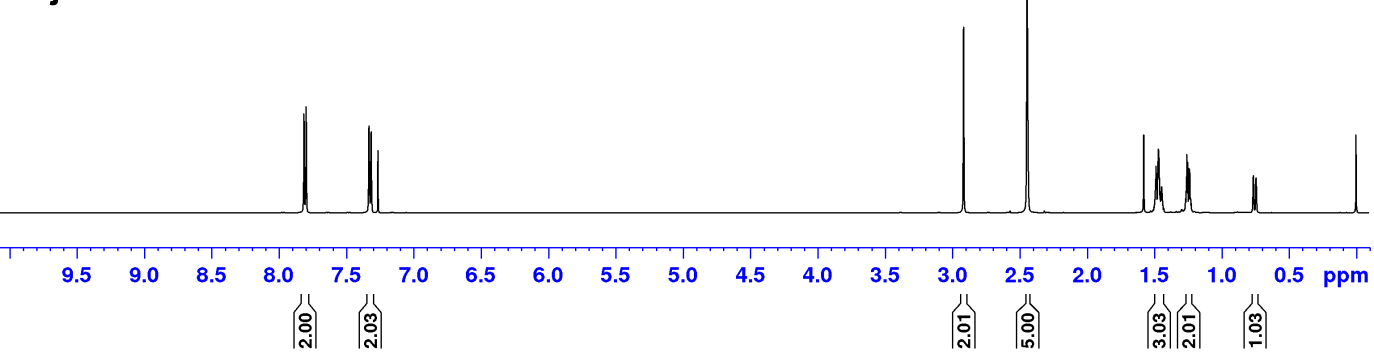

${ }^{13} \mathrm{C}\left(\mathrm{CDCl}_{3}, 125 \mathrm{MHz}\right)$

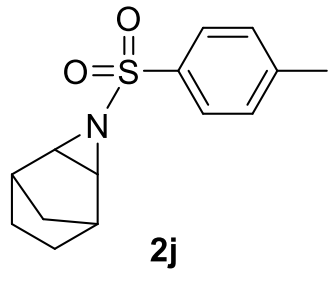

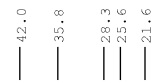

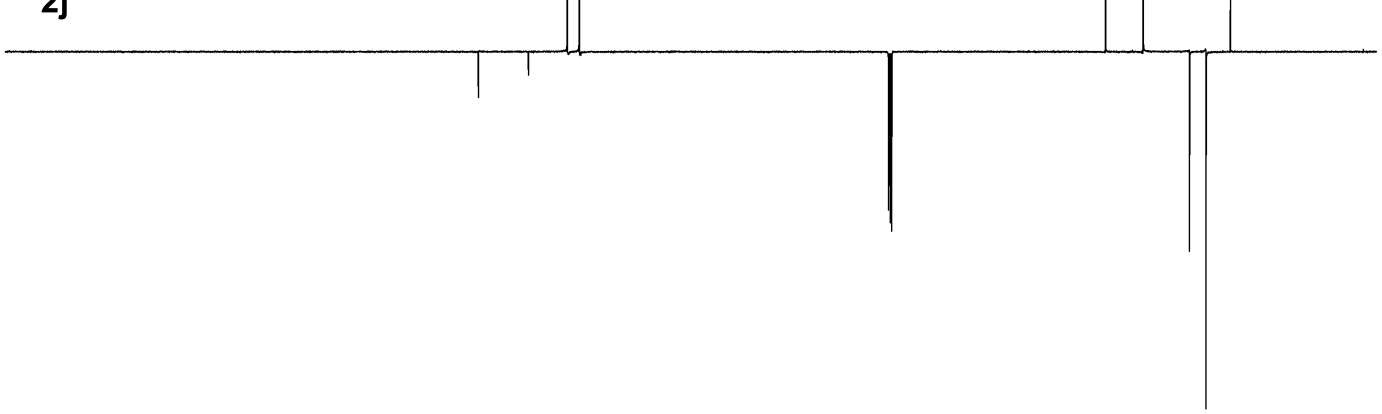

$\begin{array}{llllllllllllllllllllll}210 & 200 & 190 & 180 & 170 & 160 & 150 & 140 & 130 & 120 & 110 & 100 & 90 & 80 & 70 & 60 & 50 & 40 & 30 & 20 & 10 & \mathrm{ppm}\end{array}$ 
2k:

${ }^{1} \mathrm{H}\left(\mathrm{CDCl}_{3}, 500 \mathrm{MHz}\right)$

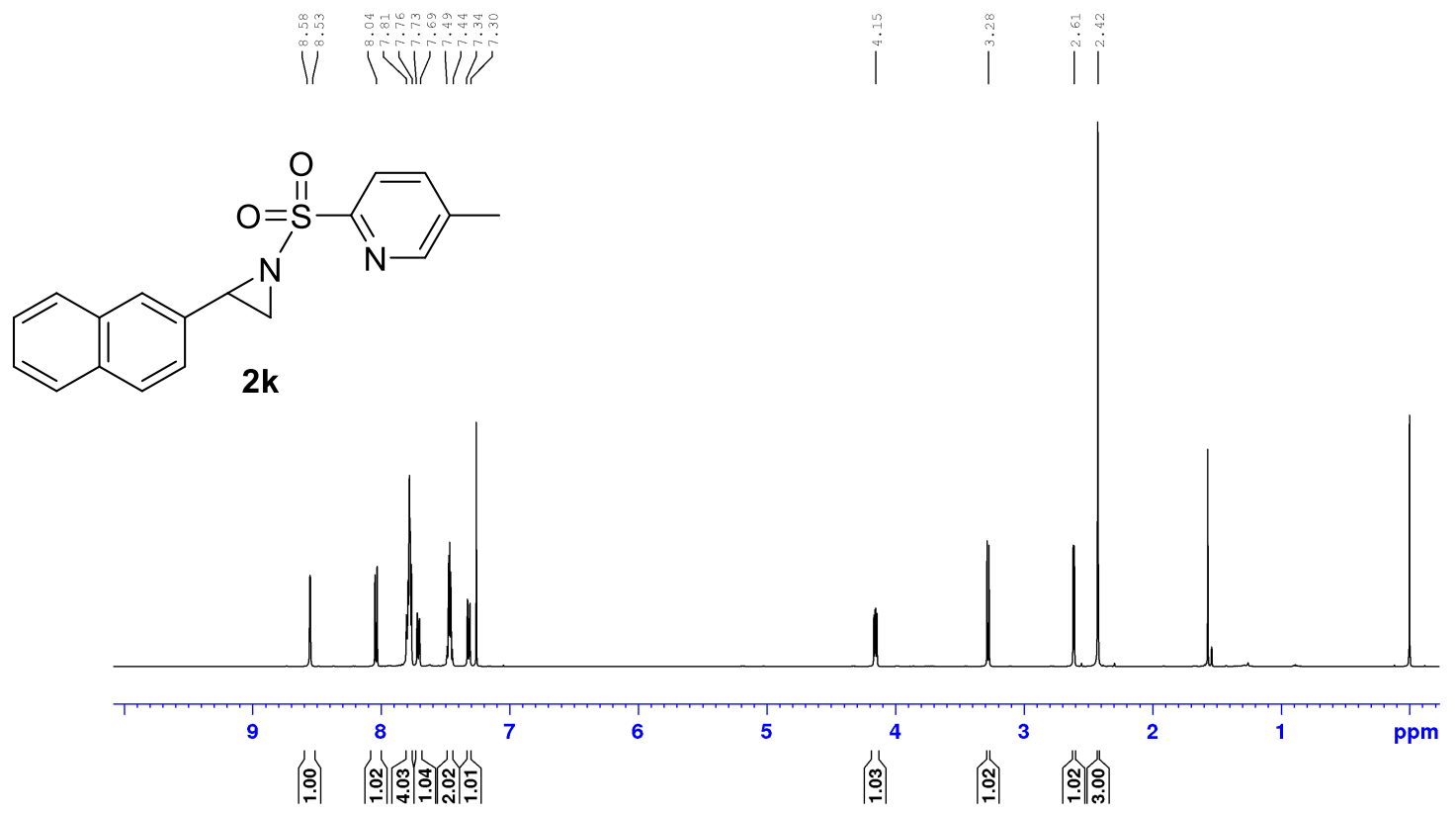

${ }^{13} \mathrm{C}\left(\mathrm{CDCl}_{3}, 125 \mathrm{MHz}\right)$

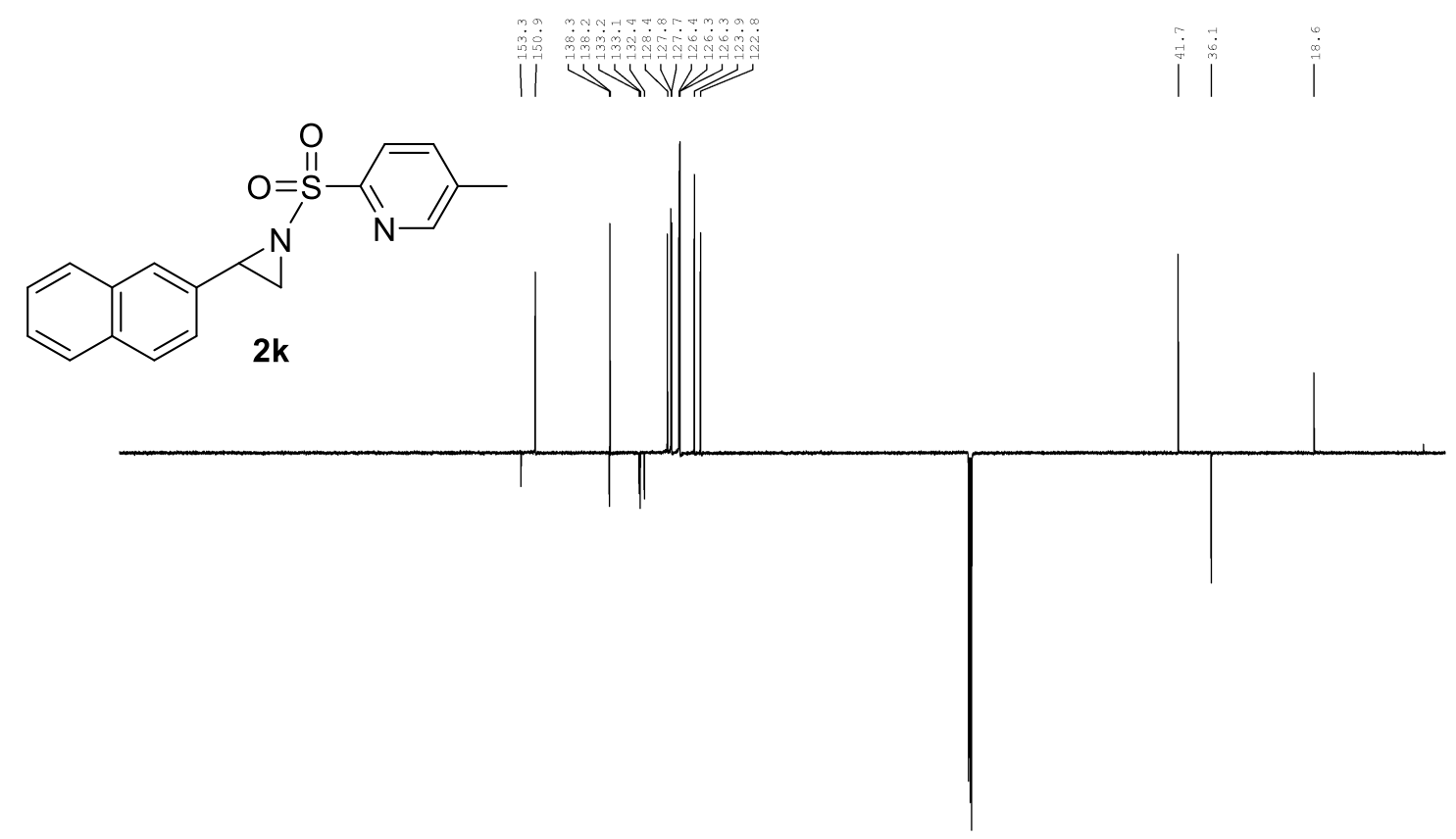

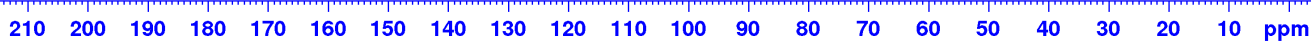


2I:

${ }^{1} \mathrm{H}\left(\mathrm{CDCl}_{3}, 500 \mathrm{MHz}\right)$

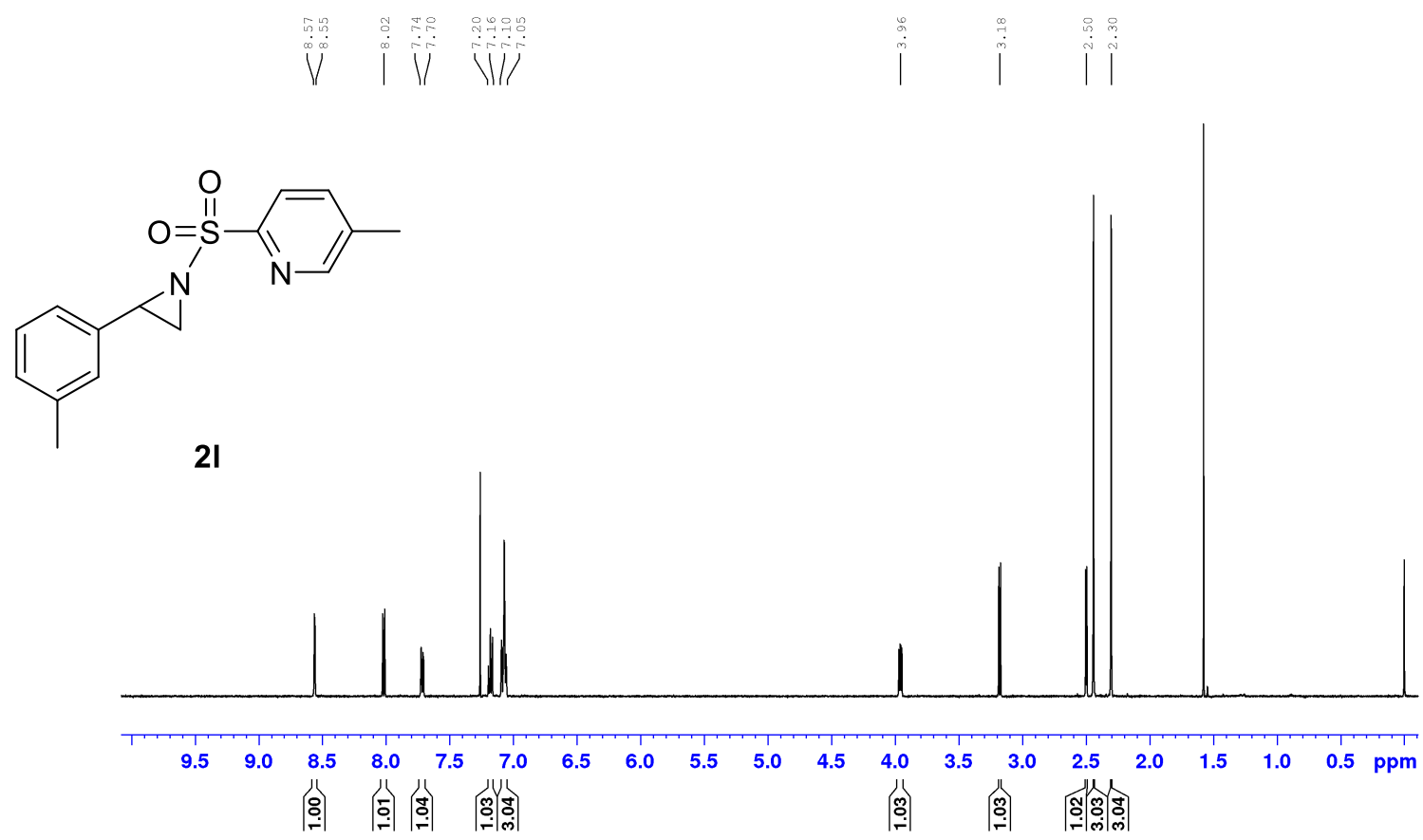

${ }^{13} \mathrm{C}\left(\mathrm{CDCl}_{3}, 125 \mathrm{MHz}\right)$
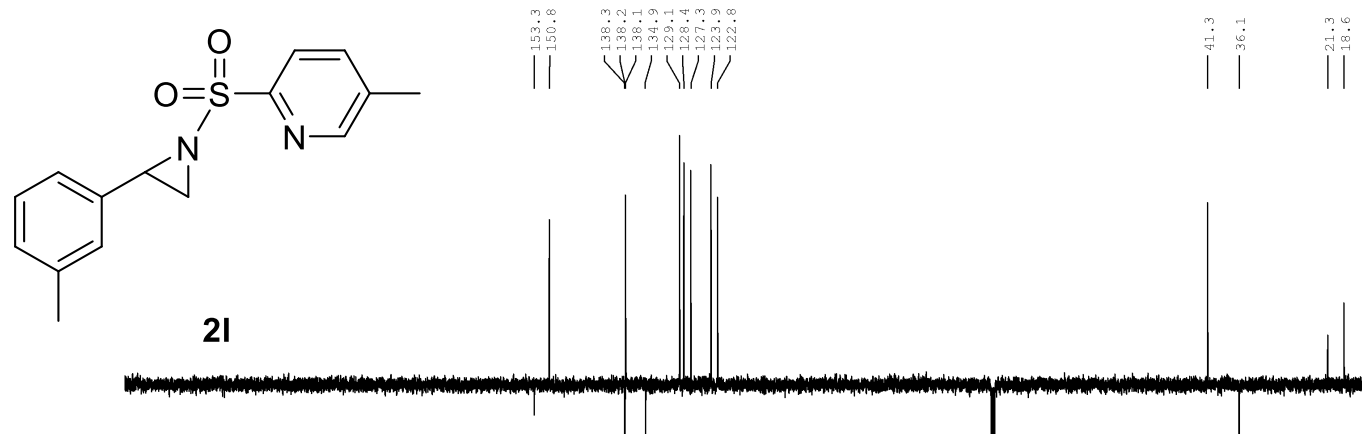

$\begin{array}{lllllllllllllllllllllll}210 & 200 & 190 & 180 & 170 & 160 & 150 & 140 & 130 & 120 & 110 & 100 & 90 & 80 & 70 & 60 & 50 & 40 & 30 & 20 & 10 & \mathrm{ppm}\end{array}$ 
3a:

${ }^{1} \mathrm{H}\left(\mathrm{CDCl}_{3}, 500 \mathrm{MHz}\right)$

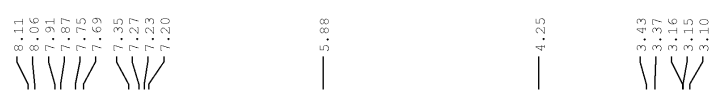<smiles>COC(CNS(=O)(=O)c1ccccc1[N+](=O)[O-])c1ccccc1</smiles>

3a

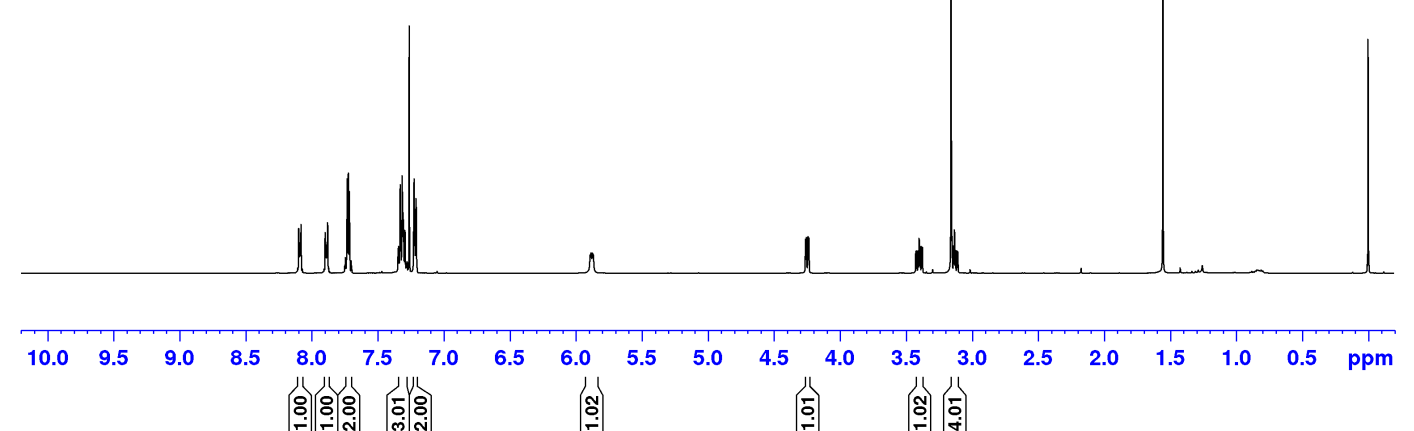

${ }^{13} \mathrm{C}\left(\mathrm{CDCl}_{3}, 125 \mathrm{MHz}\right)$<smiles>COC(CNS(=O)(=O)c1ccccc1[N+](=O)[O-])c1ccccc1</smiles>

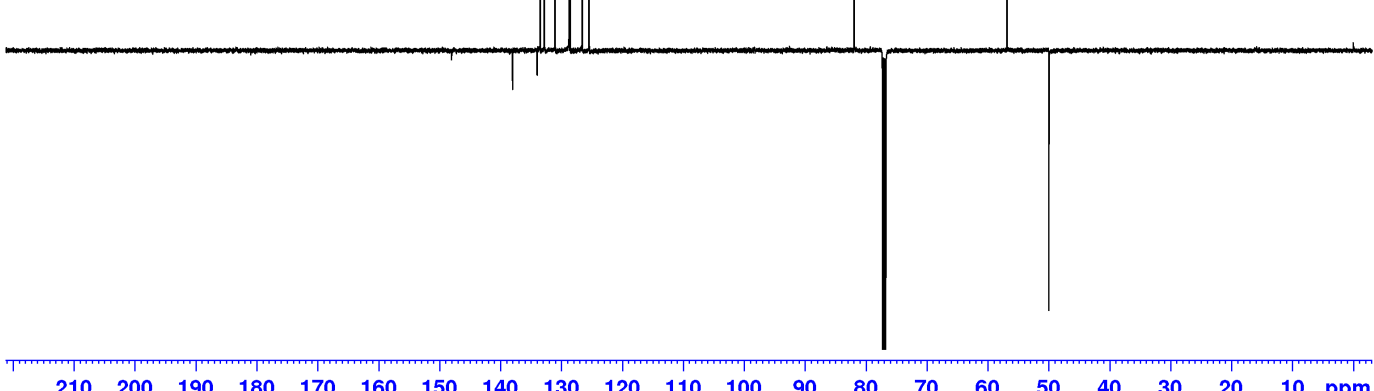


3b:

${ }^{1} \mathrm{H}\left(\mathrm{CDCl}_{3}, 500 \mathrm{MHz}\right)$

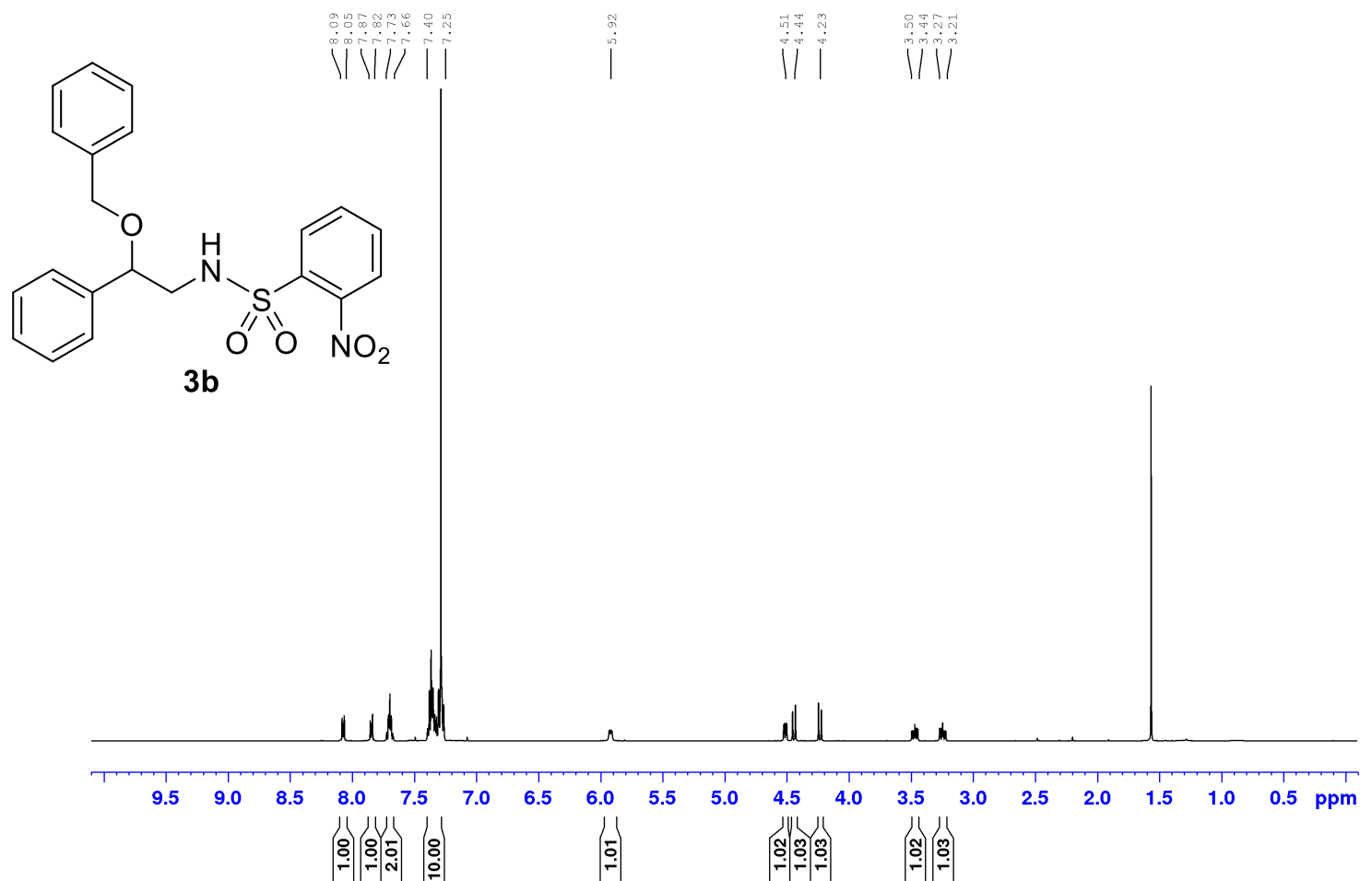

${ }^{13} \mathrm{C}\left(\mathrm{CDCl}_{3}, 125 \mathrm{MHz}\right)$<smiles>O=[N+]([O-])c1ccccc1S(=O)(=O)NCC(OCc1ccccc1)c1ccccc1</smiles>
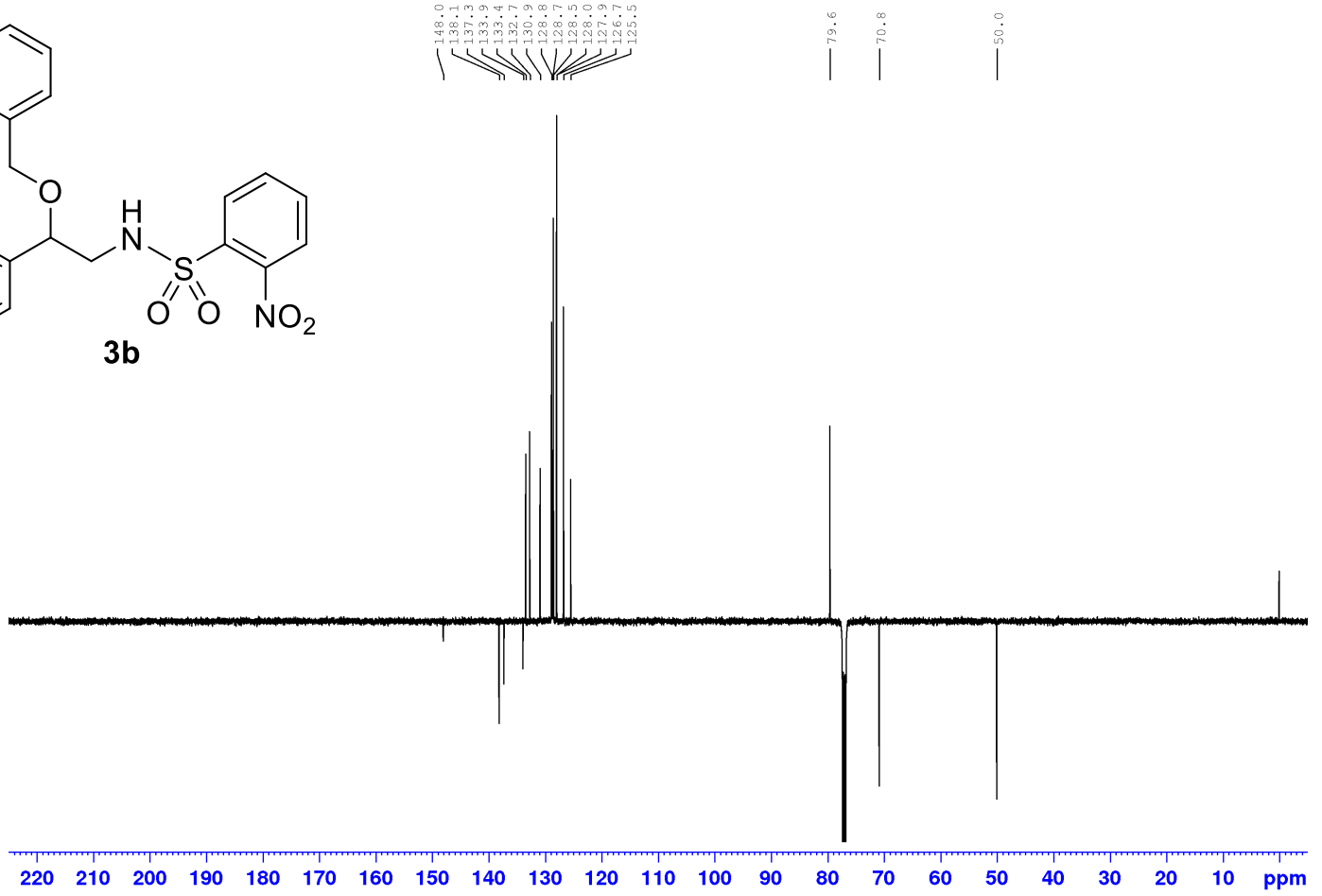
3c:

${ }^{1} \mathrm{H}\left(\mathrm{CDCl}_{3}, 500 \mathrm{MHz}\right)$

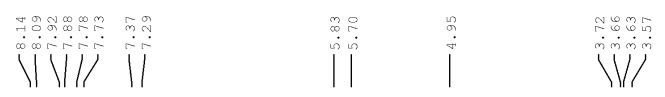<smiles>O=[N+]([O-])c1ccccc1S(=O)(=O)NCC(Cl)c1ccccc1</smiles>

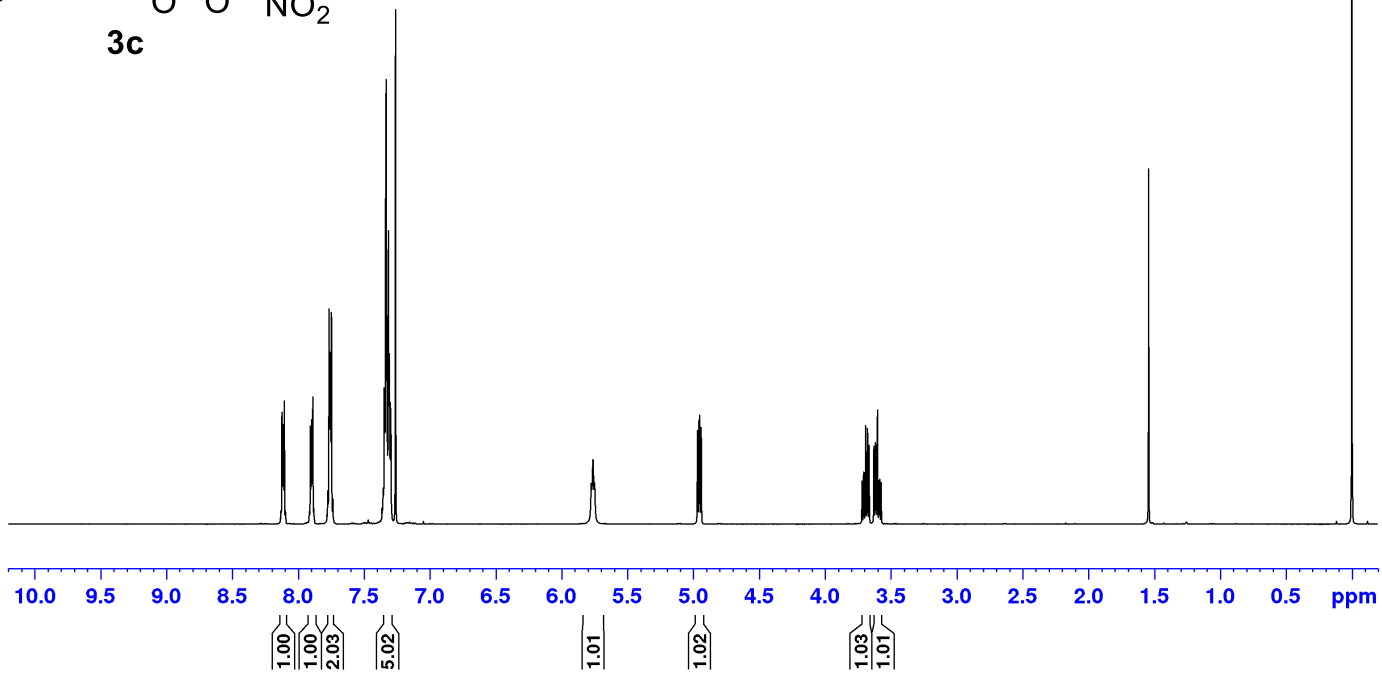

${ }^{13} \mathrm{C}\left(\mathrm{CDCl}_{3}, 125 \mathrm{MHz}\right)$<smiles>O=[N+]([O-])c1ccccc1S(=O)(=O)NCC(Cl)c1ccccc1</smiles>

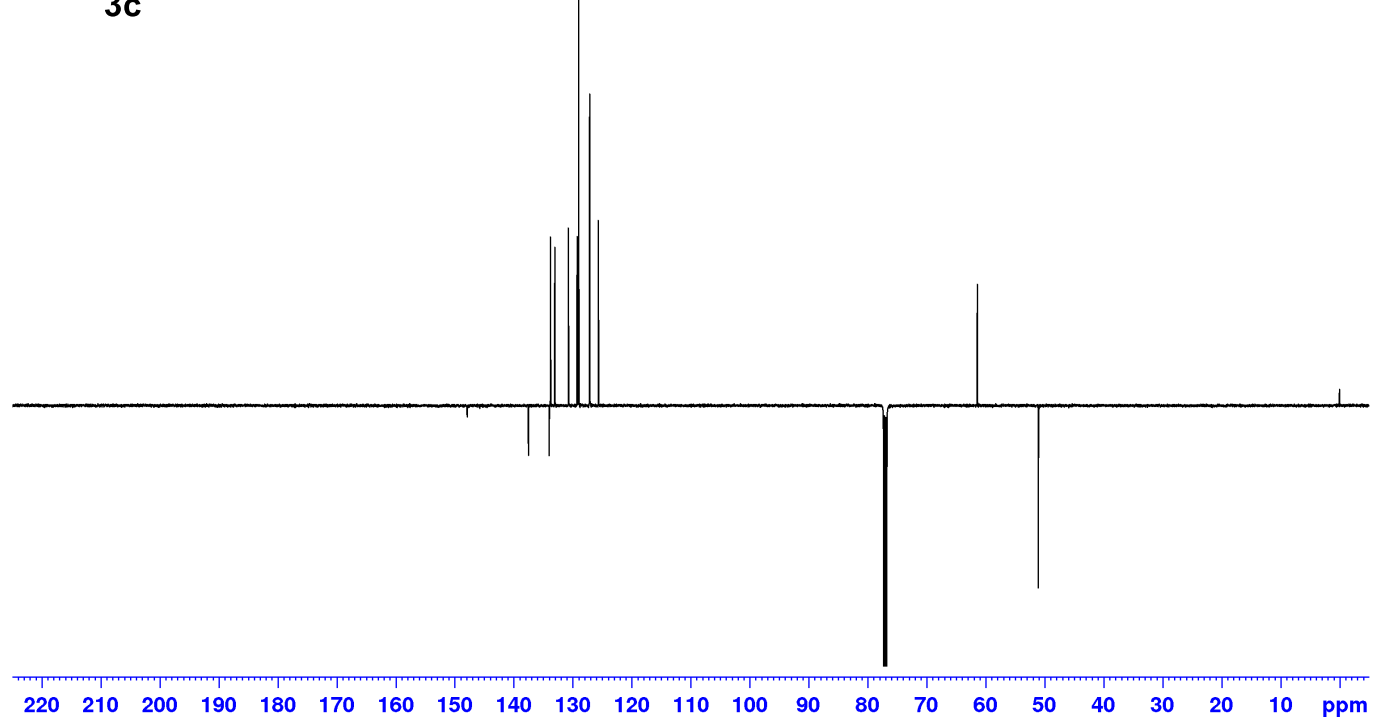


3d:

${ }^{1} \mathrm{H}\left(\mathrm{CDCl}_{3}, 500 \mathrm{MHz}\right)$

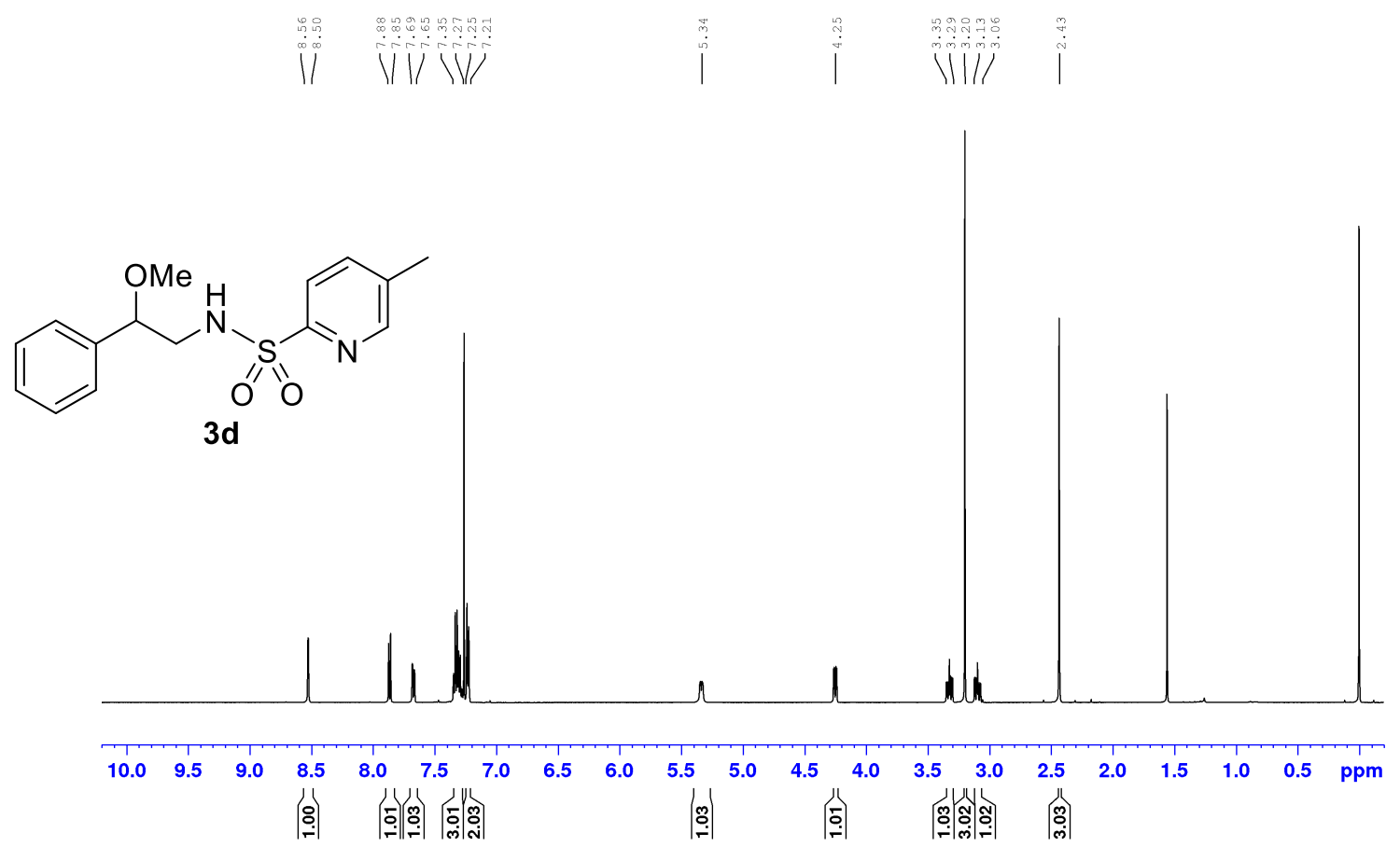

${ }^{13} \mathrm{C}\left(\mathrm{CDCl}_{3}, 125 \mathrm{MHz}\right)$
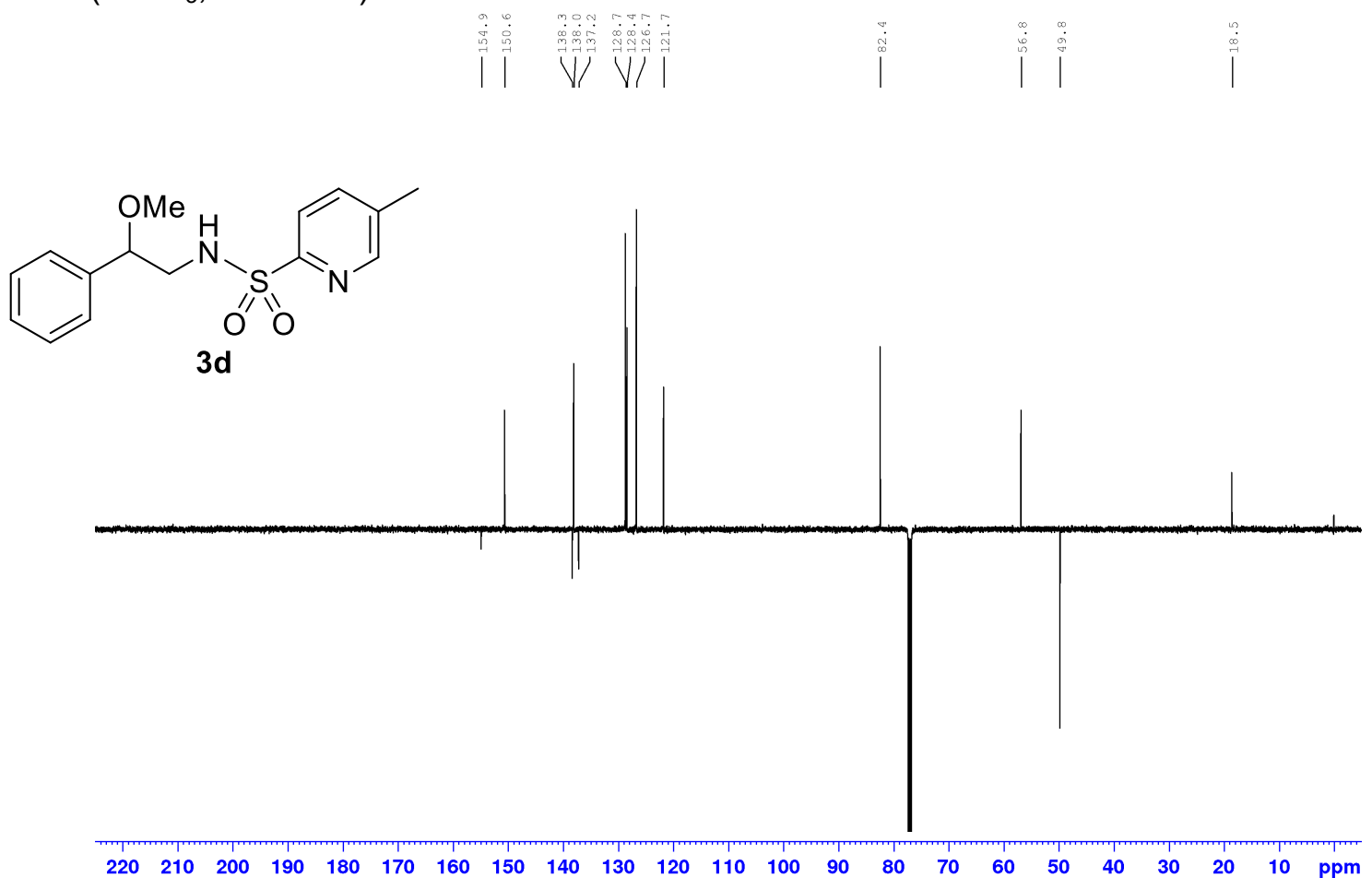
3e:

${ }^{1} \mathrm{H}\left(\mathrm{CD}_{2} \mathrm{Cl}_{2}, 500 \mathrm{MHz}\right)$

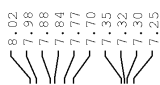

(N)

$3 e$

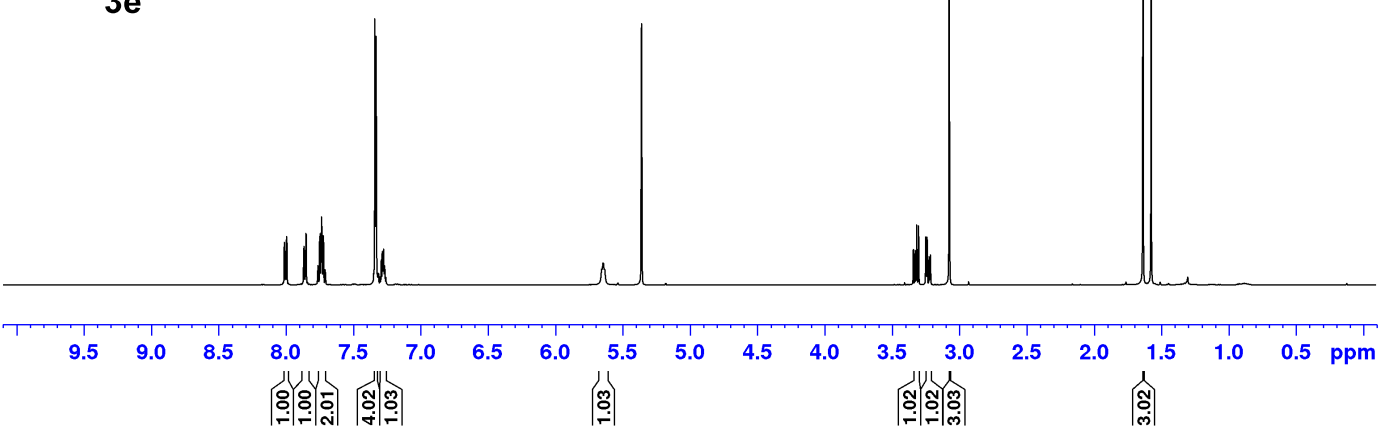

${ }^{13} \mathrm{C}\left(\mathrm{CD}_{2} \mathrm{Cl}_{2}, 125 \mathrm{MHz}\right)$

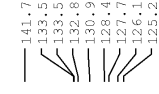<smiles>COC(C)(CNS(=O)(=O)c1ccccc1[N+](=O)[O-])c1ccccc1</smiles>

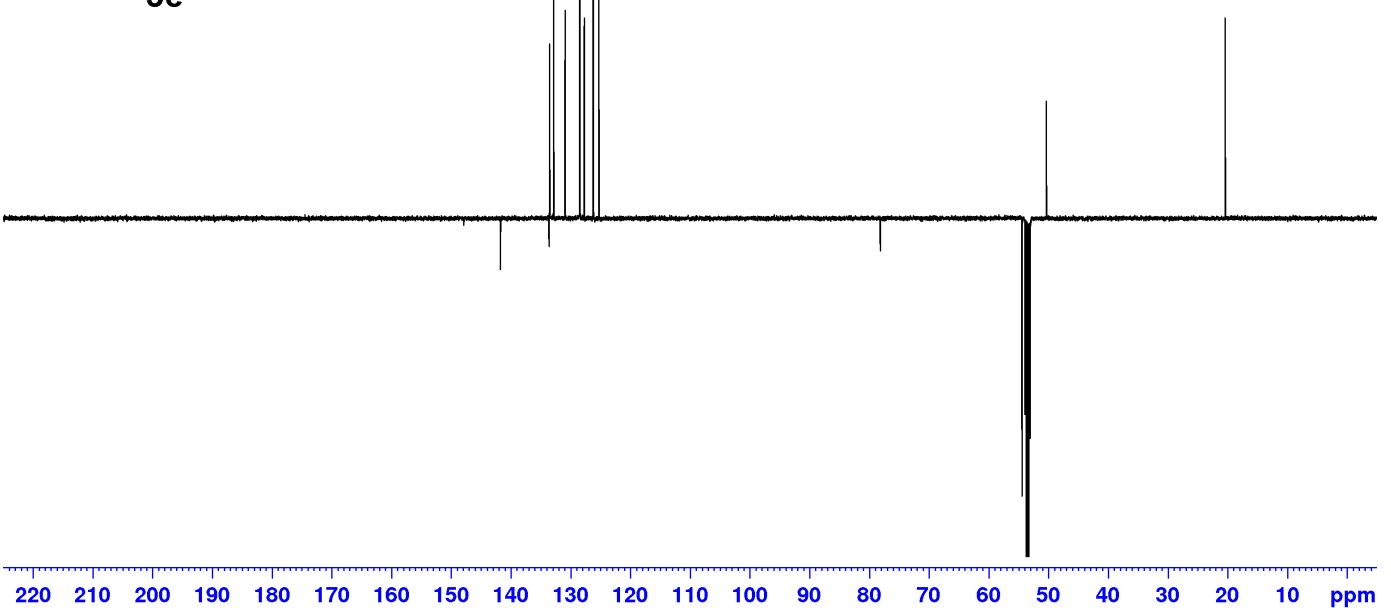


3f:

${ }^{1} \mathrm{H}\left(\mathrm{CD}_{3} \mathrm{CN}, 500 \mathrm{MHz}\right)$

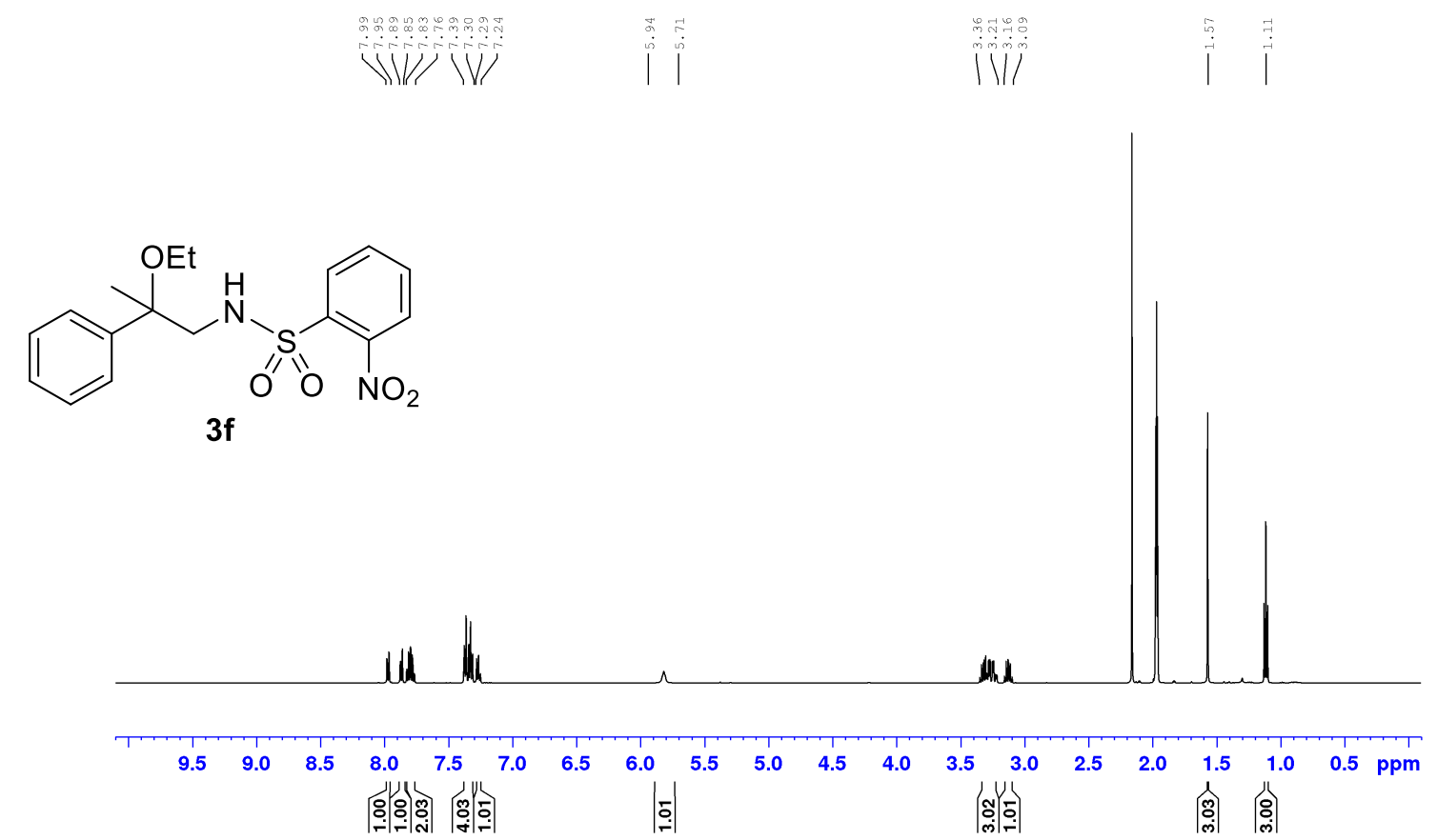

${ }^{13} \mathrm{C}\left(\mathrm{CD}_{3} \mathrm{CN}, 125 \mathrm{MHz}\right)$

$\underbrace{\mathrm{O}}_{3 \mathrm{O}}$
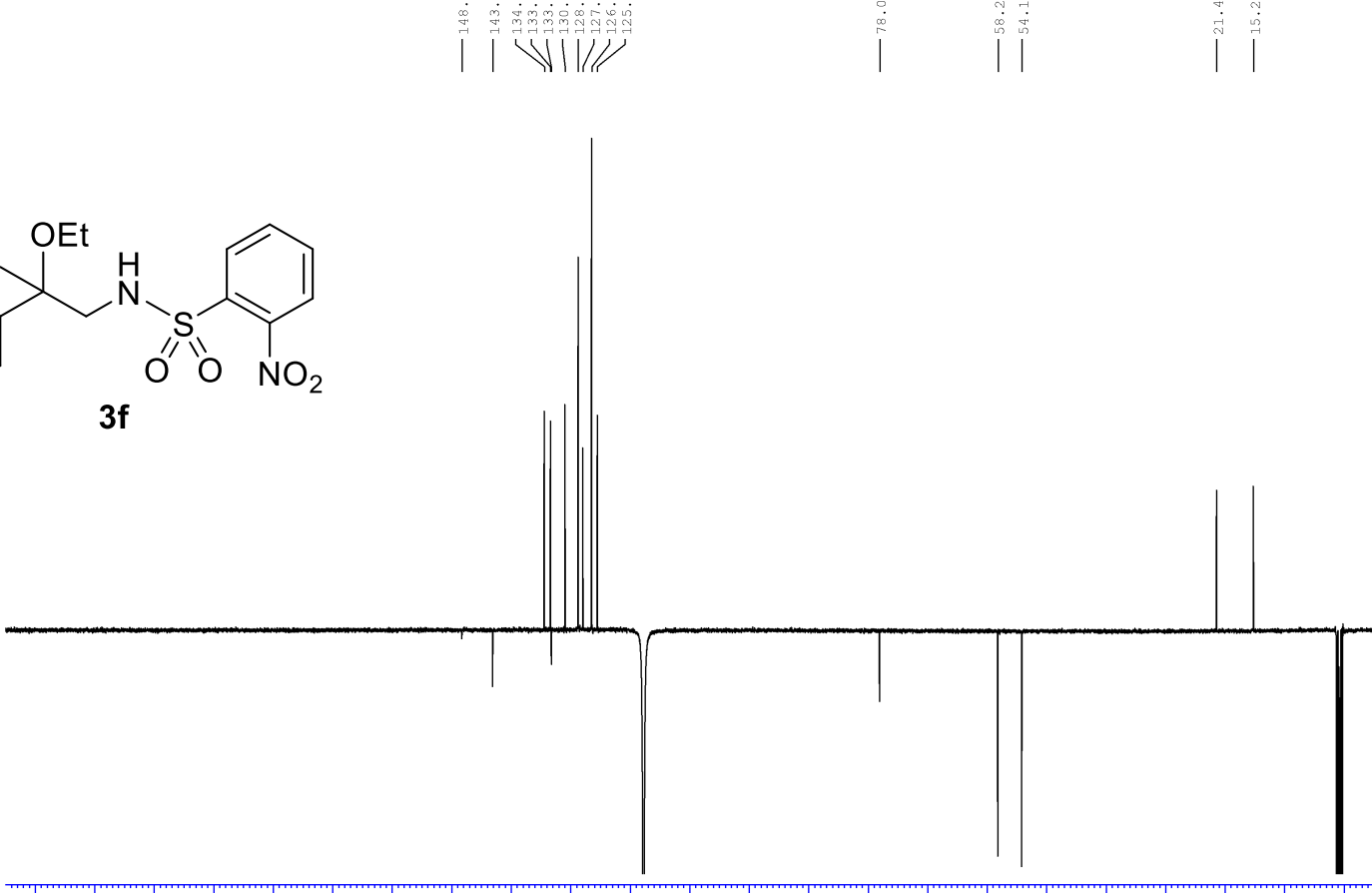

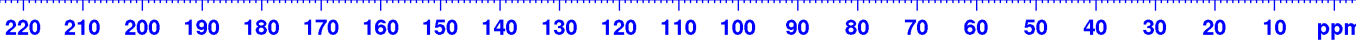


3g:

${ }^{1} \mathrm{H}\left(\mathrm{CDCl}_{3}, 500 \mathrm{MHz}\right)$

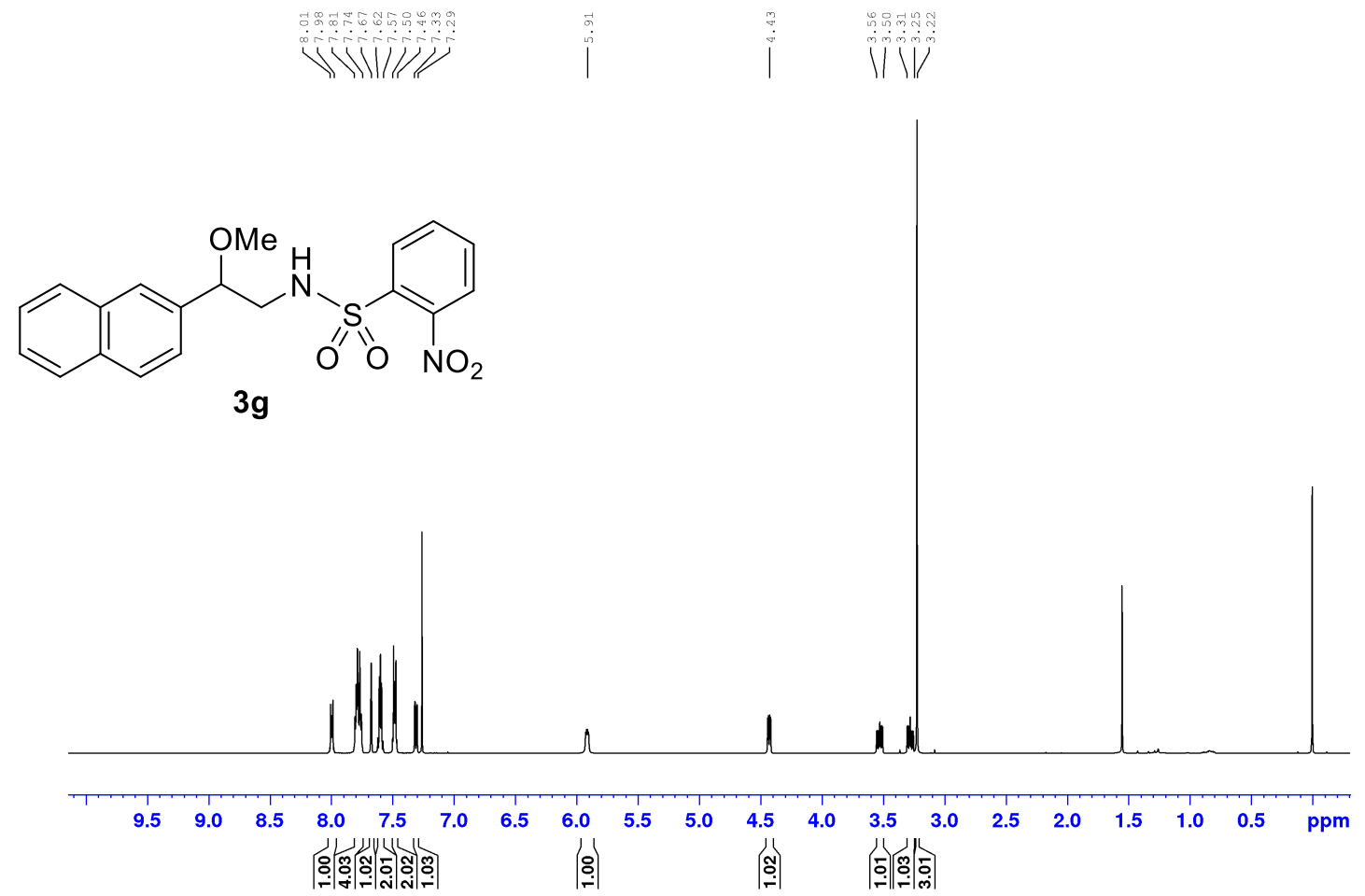

${ }^{13} \mathrm{C}\left(\mathrm{CDCl}_{3}, 125 \mathrm{MHz}\right)$<smiles>COC(CNS(=O)(=O)c1ccccc1[N+](=O)[O-])c1ccc2ccccc2c1</smiles>

$3 g$
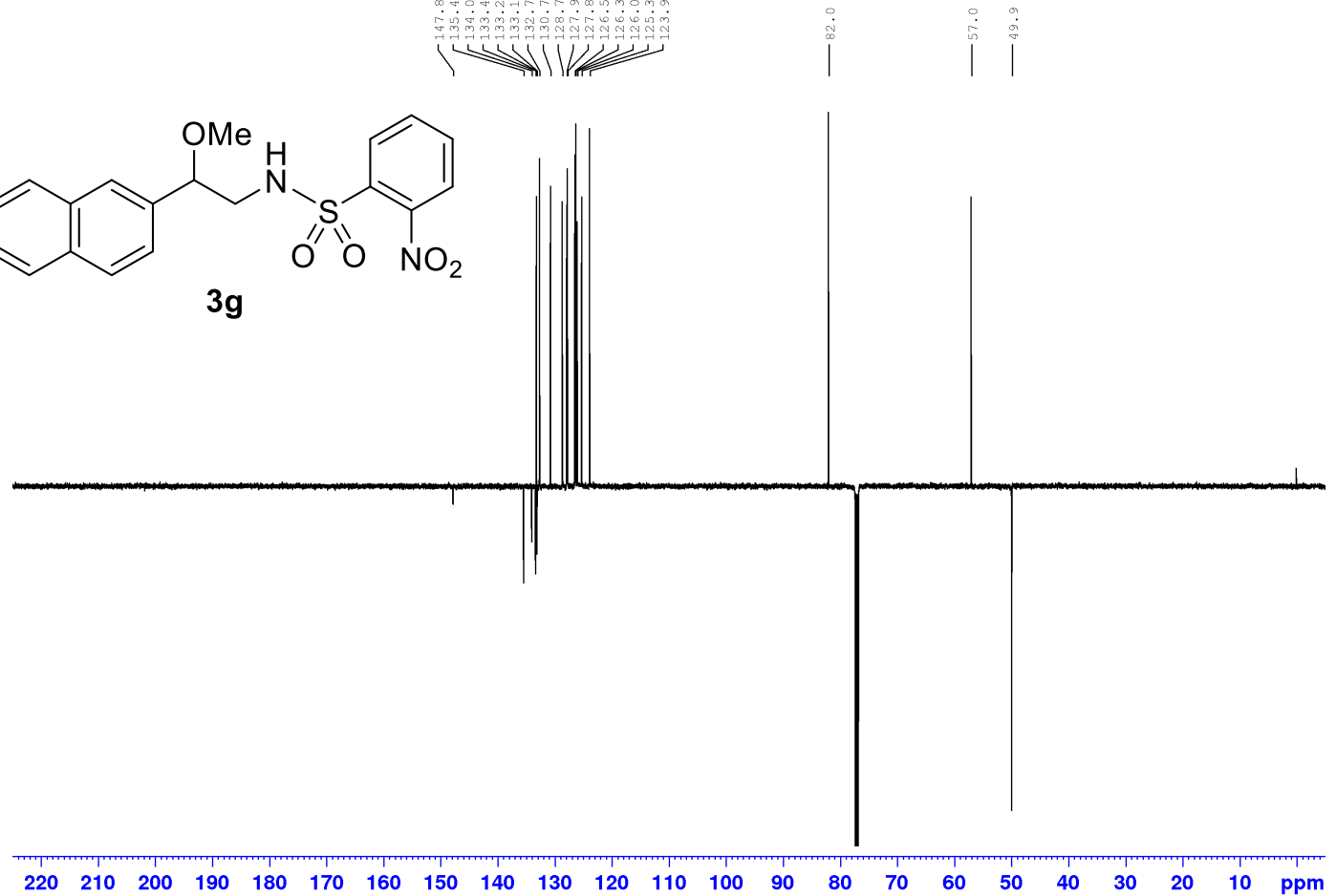
3h:

${ }^{1} \mathrm{H}\left(\mathrm{CDCl}_{3}, 500 \mathrm{MHz}\right)$

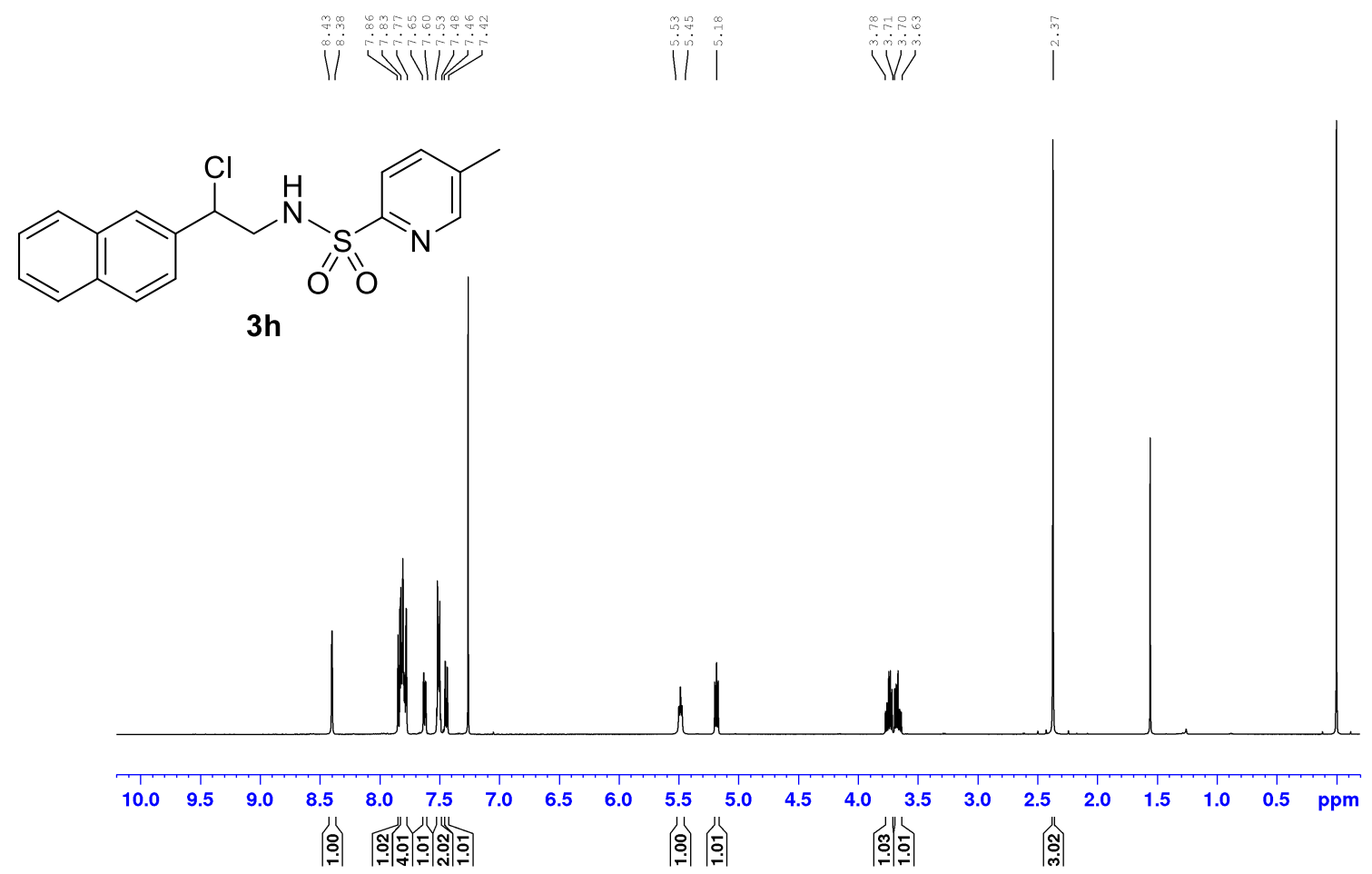

${ }^{13} \mathrm{C}\left(\mathrm{CDCl}_{3}, 125 \mathrm{MHz}\right)$<smiles>Cc1ccc(S(=O)(=O)NCC(Cl)c2ccc3ccccc3c2)nc1</smiles>

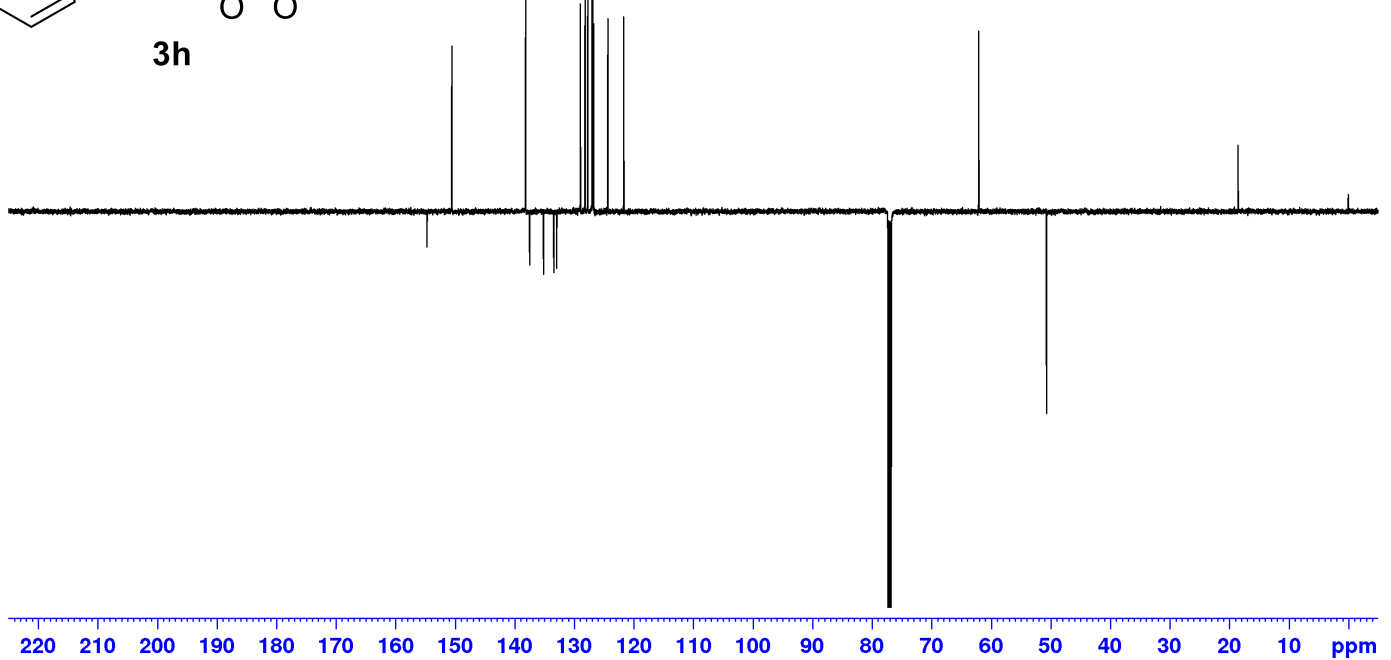


3i:

${ }^{1} \mathrm{H}\left(\mathrm{CDCl}_{3}, 500 \mathrm{MHz}\right)$

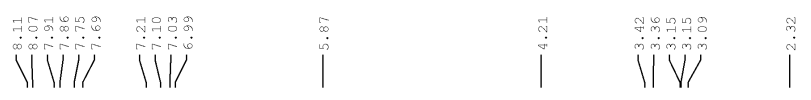<smiles>COC(CNS(=O)(=O)c1ccccc1[N+](=O)[O-])c1cccc(C)c1</smiles>

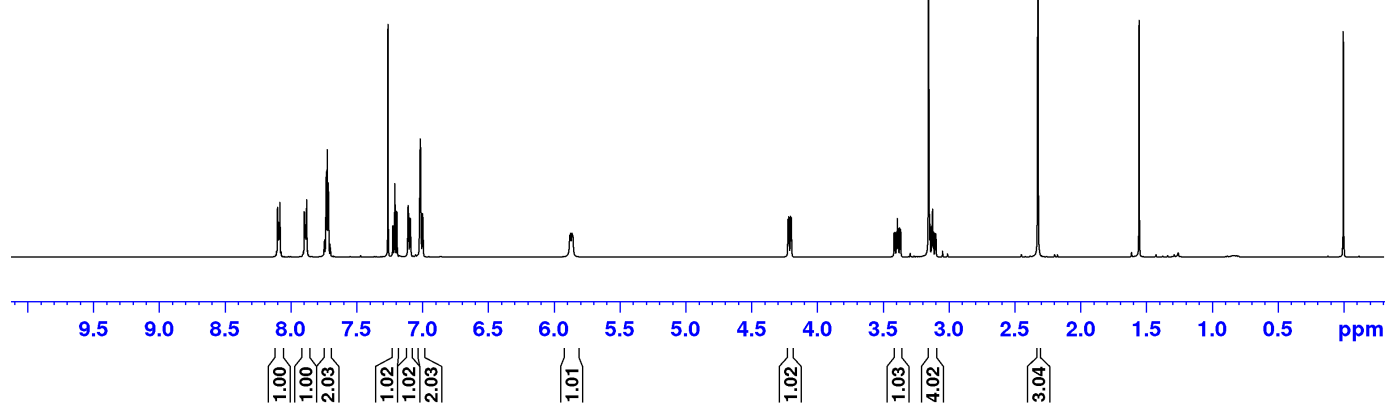

${ }^{13} \mathrm{C}\left(\mathrm{CDCl}_{3}, 125 \mathrm{MHz}\right)$<smiles>COC(CNS(=O)(=O)c1ccccc1[N+](=O)[O-])c1cccc(C)c1</smiles>
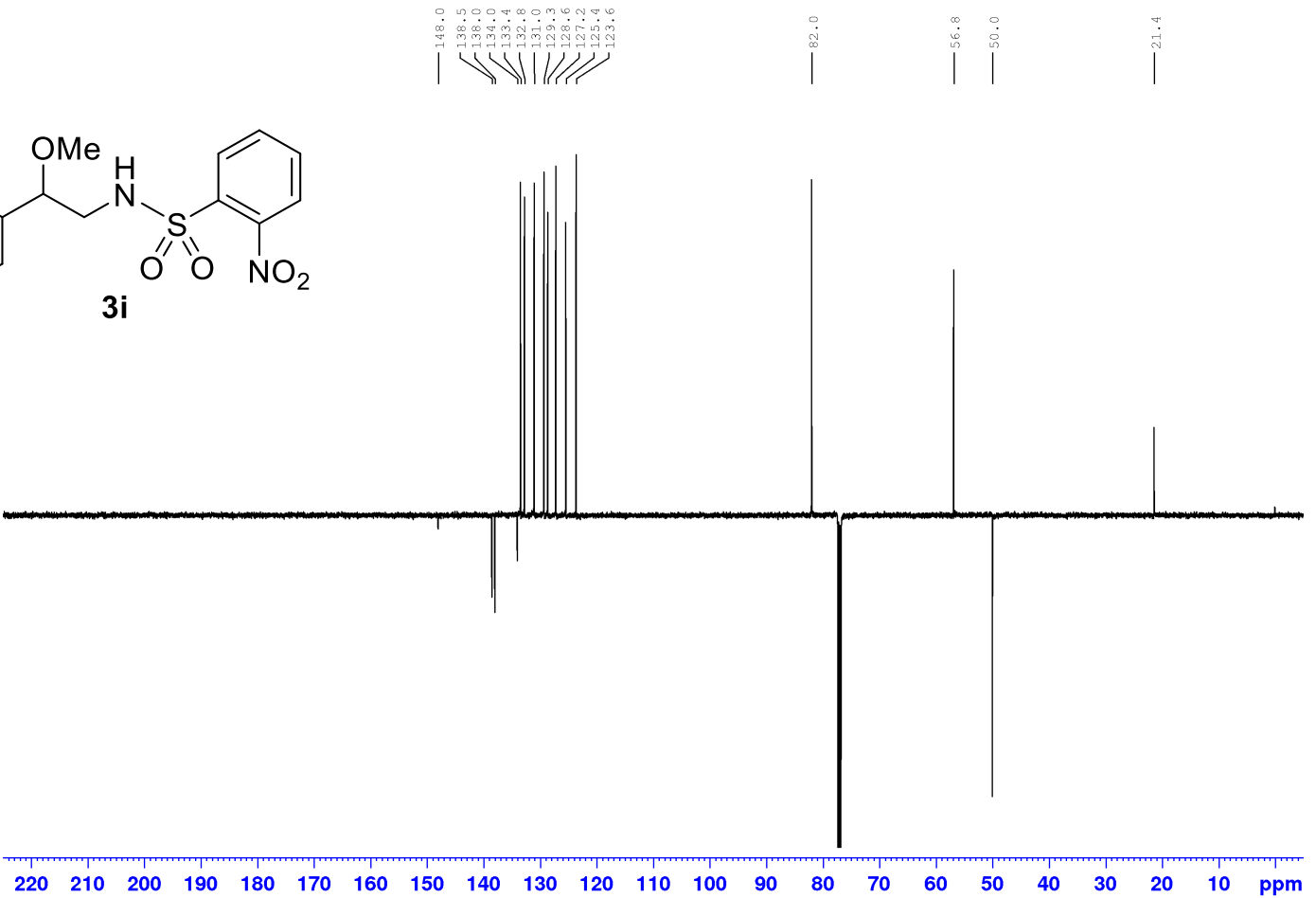
3j:

${ }^{1} \mathrm{H}\left(\mathrm{CDCl}_{3}, 500 \mathrm{MHz}\right)$

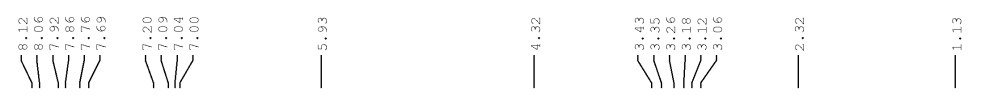<smiles>CCOC(CNS(=O)(=O)c1ccccc1[N+](=O)[O-])c1cccc(C)c1</smiles>

3j

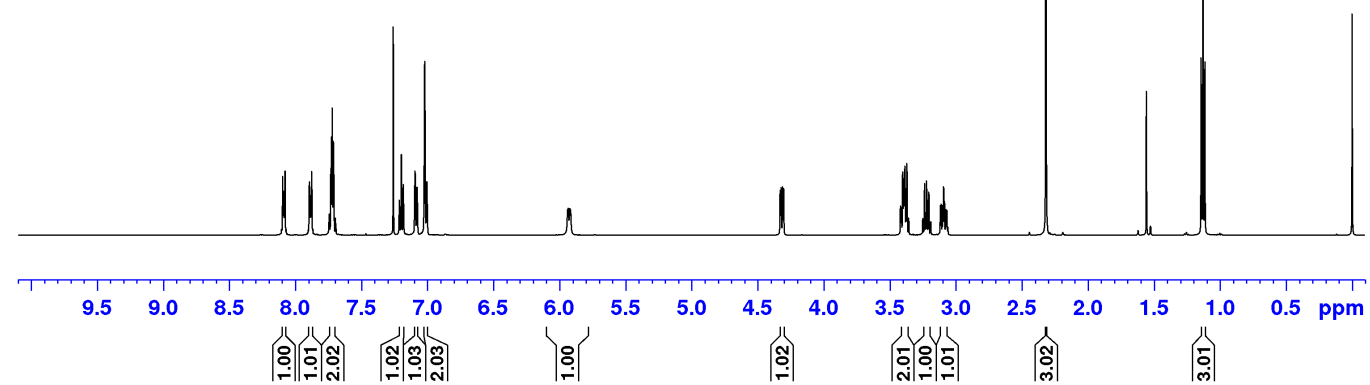

${ }^{13} \mathrm{C}\left(\mathrm{CDCl}_{3}, 125 \mathrm{MHz}\right)$<smiles>CCOC(CNS(=O)(=O)c1ccccc1[N+](=O)[O-])c1cccc(C)c1</smiles>

Vullill

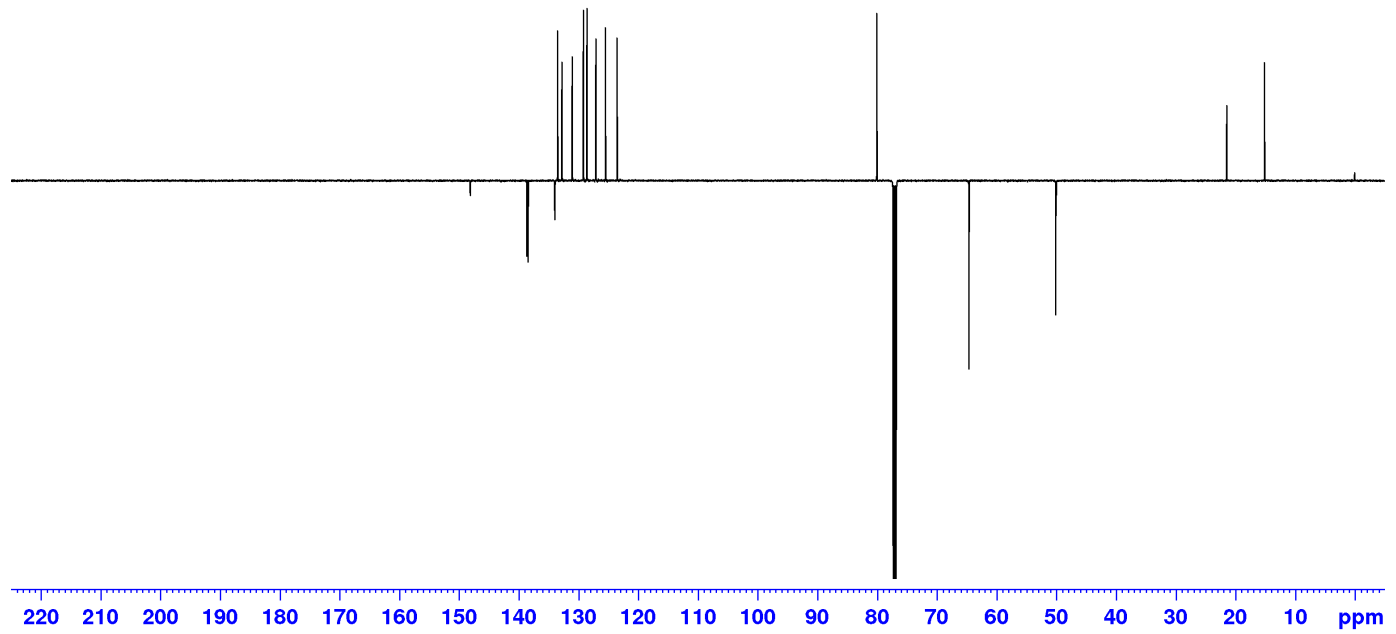


3k:

${ }^{1} \mathrm{H}\left(\mathrm{CDCl}_{3}, 500 \mathrm{MHz}\right)$

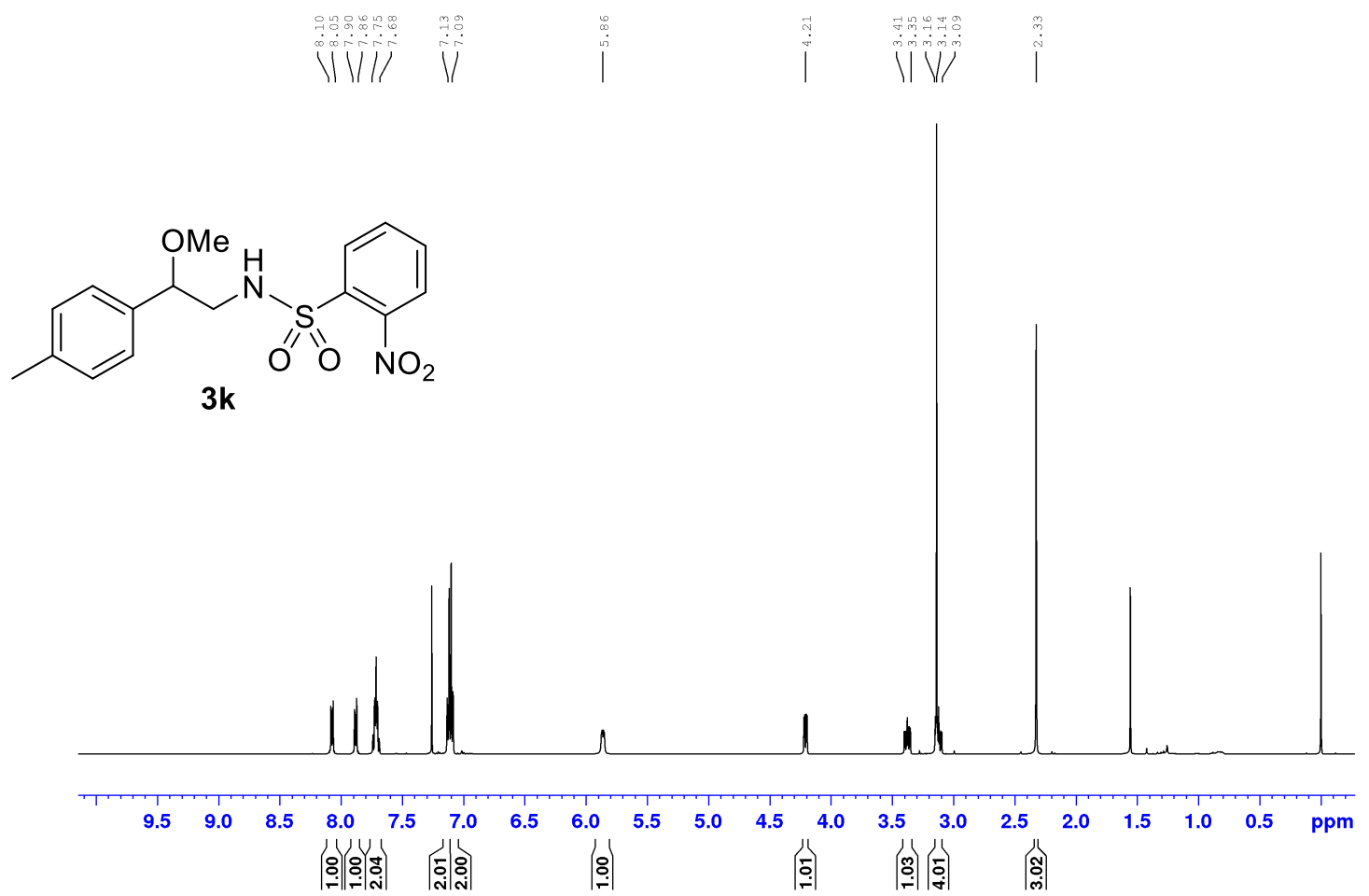

${ }^{13} \mathrm{C}\left(\mathrm{CDCl}_{3}, 125 \mathrm{MHz}\right)$
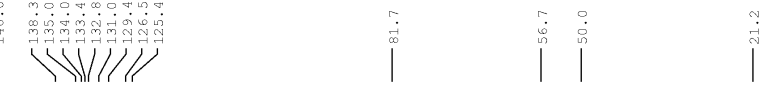<smiles>COC(CNS(=O)(=O)c1ccccc1[N+](=O)[O-])c1ccc(C)cc1</smiles>

$\begin{array}{lllllllllllllllllllllll}220 & 210 & 200 & 190 & 180 & 170 & 160 & 150 & 140 & 130 & 120 & 110 & 100 & 90 & 80 & 70 & 60 & 50 & 40 & 30 & 20 & 10 & \mathrm{ppm}\end{array}$ 
3I:

${ }^{1} \mathrm{H}\left(\mathrm{CDCl}_{3}, 500 \mathrm{MHz}\right)$

VIVII<smiles>Cc1ccc(C(Cl)CNS(=O)(=O)c2ccccc2[N+](=O)[O-])cc1</smiles>

31

:

${ }^{13} \mathrm{C}\left(\mathrm{CDCl}_{3}, 125 \mathrm{MHz}\right)$<smiles>Cc1ccc(C(Cl)CNS(=O)(=O)c2ccccc2[N+](=O)[O-])cc1</smiles>
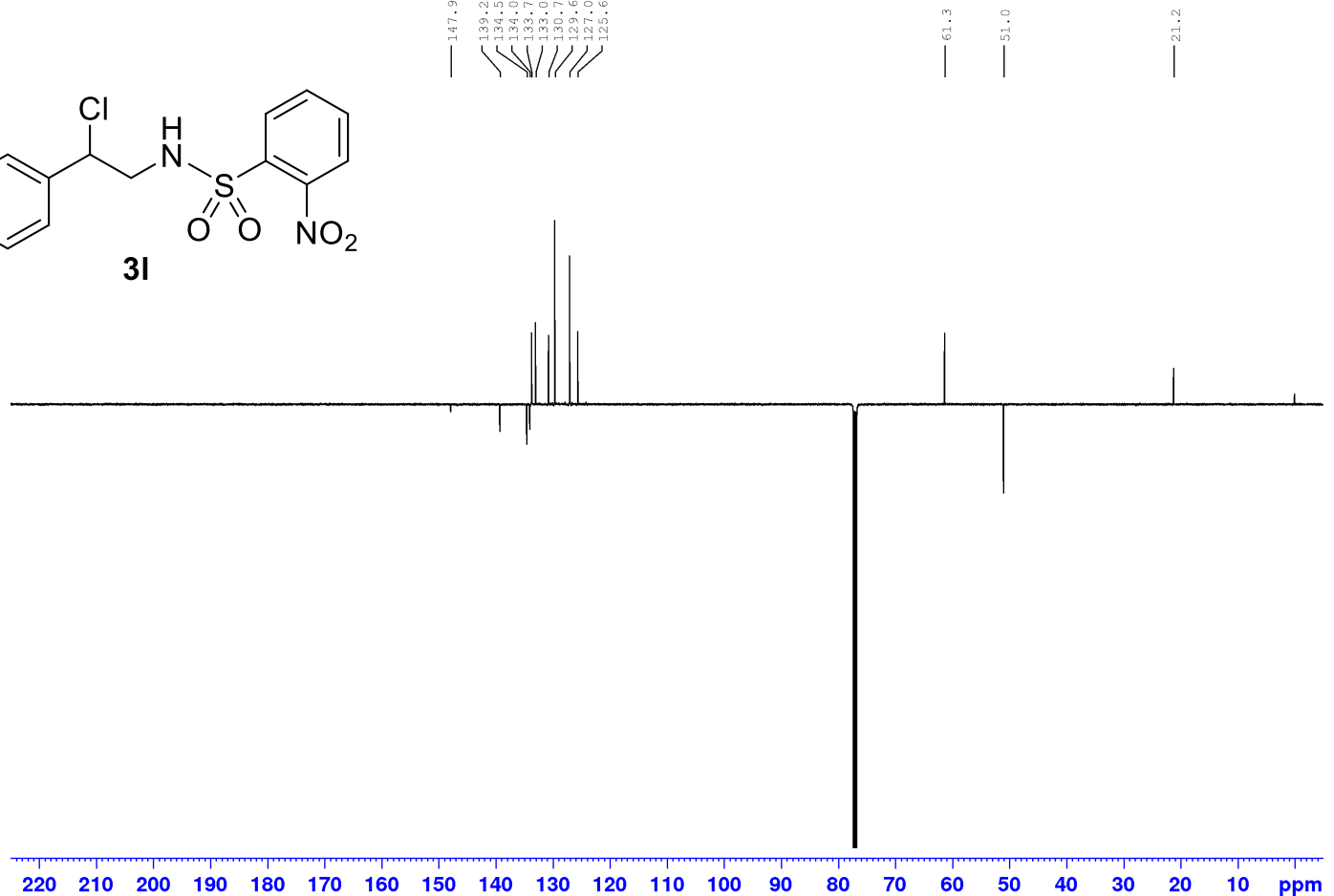
3m:

${ }^{1} \mathrm{H}\left(\mathrm{CDCl}_{3}, 500 \mathrm{MHz}\right)$

VIVII<smiles>COC(CNS(=O)(=O)c1ccccc1[N+](=O)[O-])c1ccc(Cl)cc1</smiles>

$3 \mathrm{~m}$

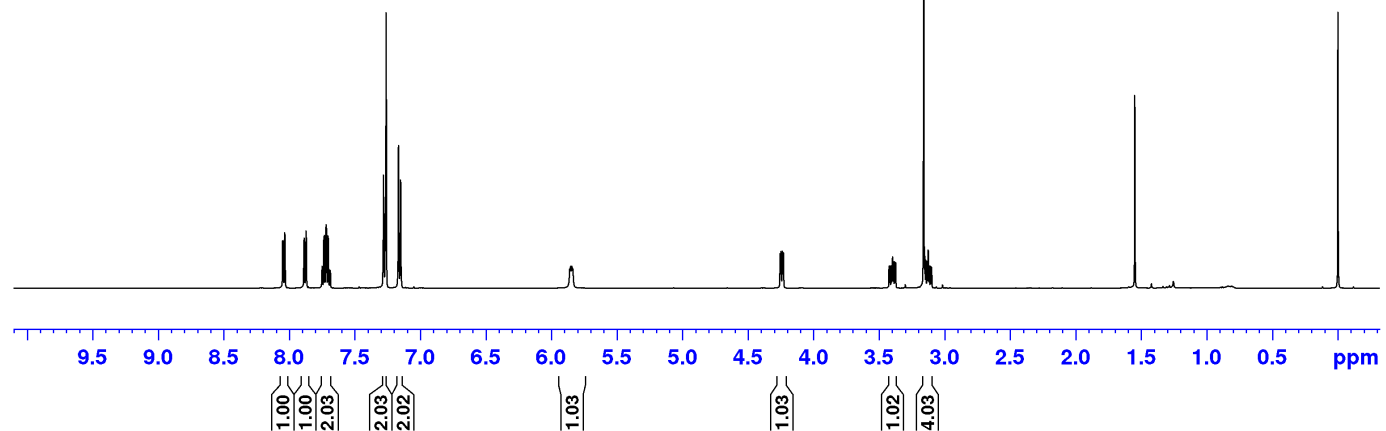

${ }^{13} \mathrm{C}\left(\mathrm{CDCl}_{3}, 125 \mathrm{MHz}\right)$

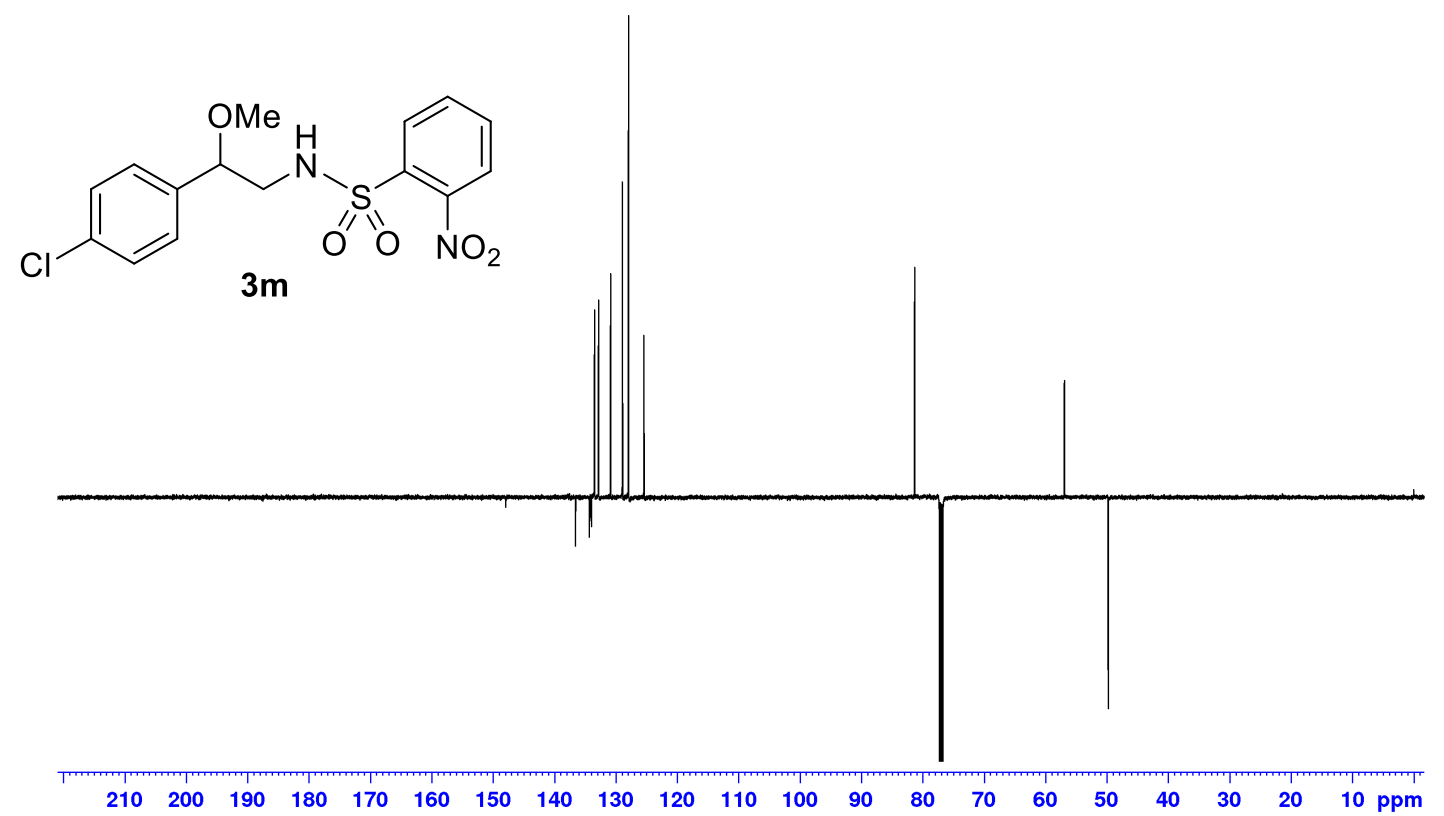


3n:

${ }^{1} \mathrm{H}\left(\mathrm{CDCl}_{3}, 500 \mathrm{MHz}\right)$

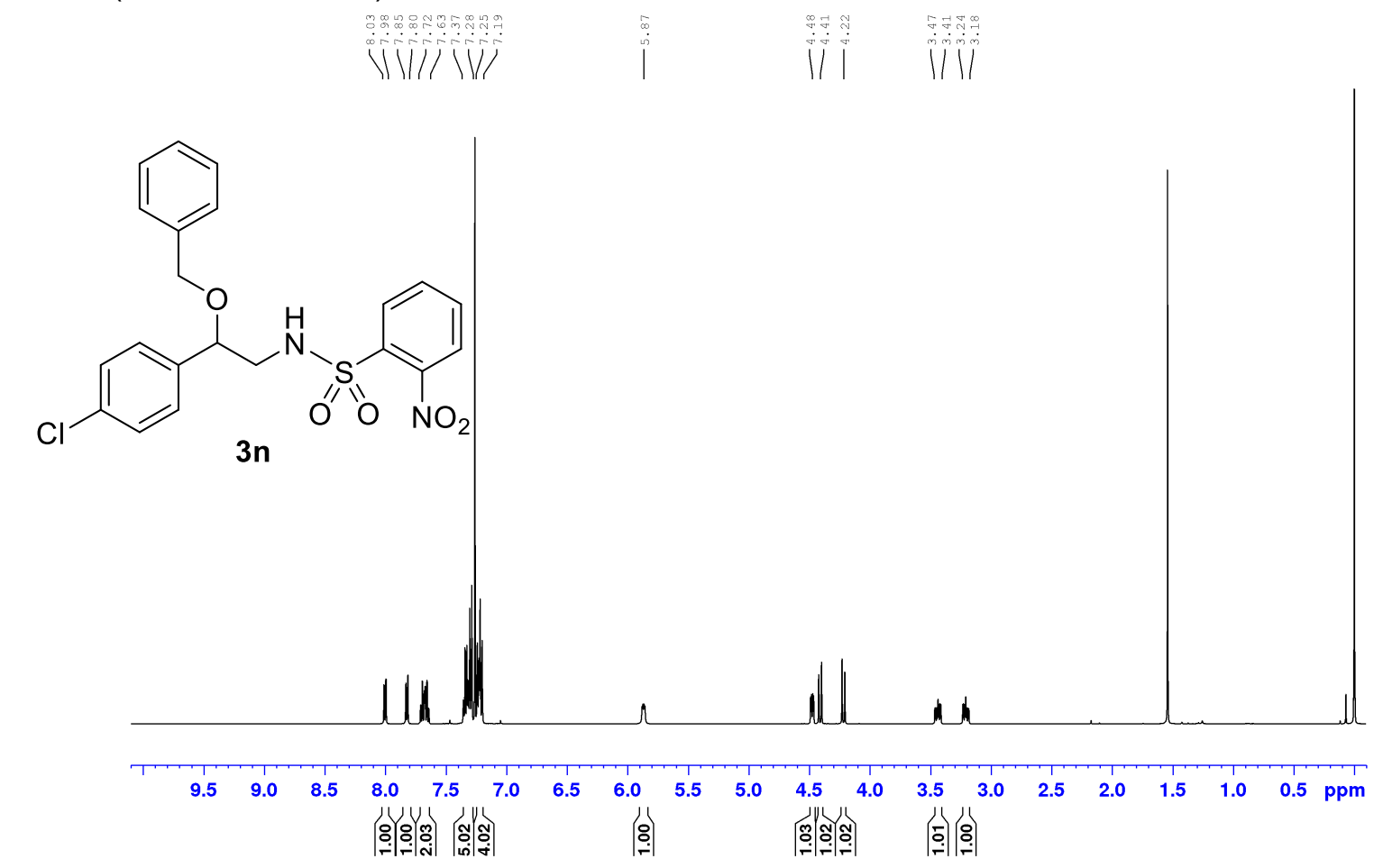

${ }^{13} \mathrm{C}\left(\mathrm{CDCl}_{3}, 125 \mathrm{MHz}\right)$<smiles>O=[N+]([O-])c1ccccc1S(=O)(=O)NCC(OCc1ccccc1)c1ccc(Cl)cc1</smiles>

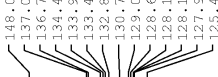
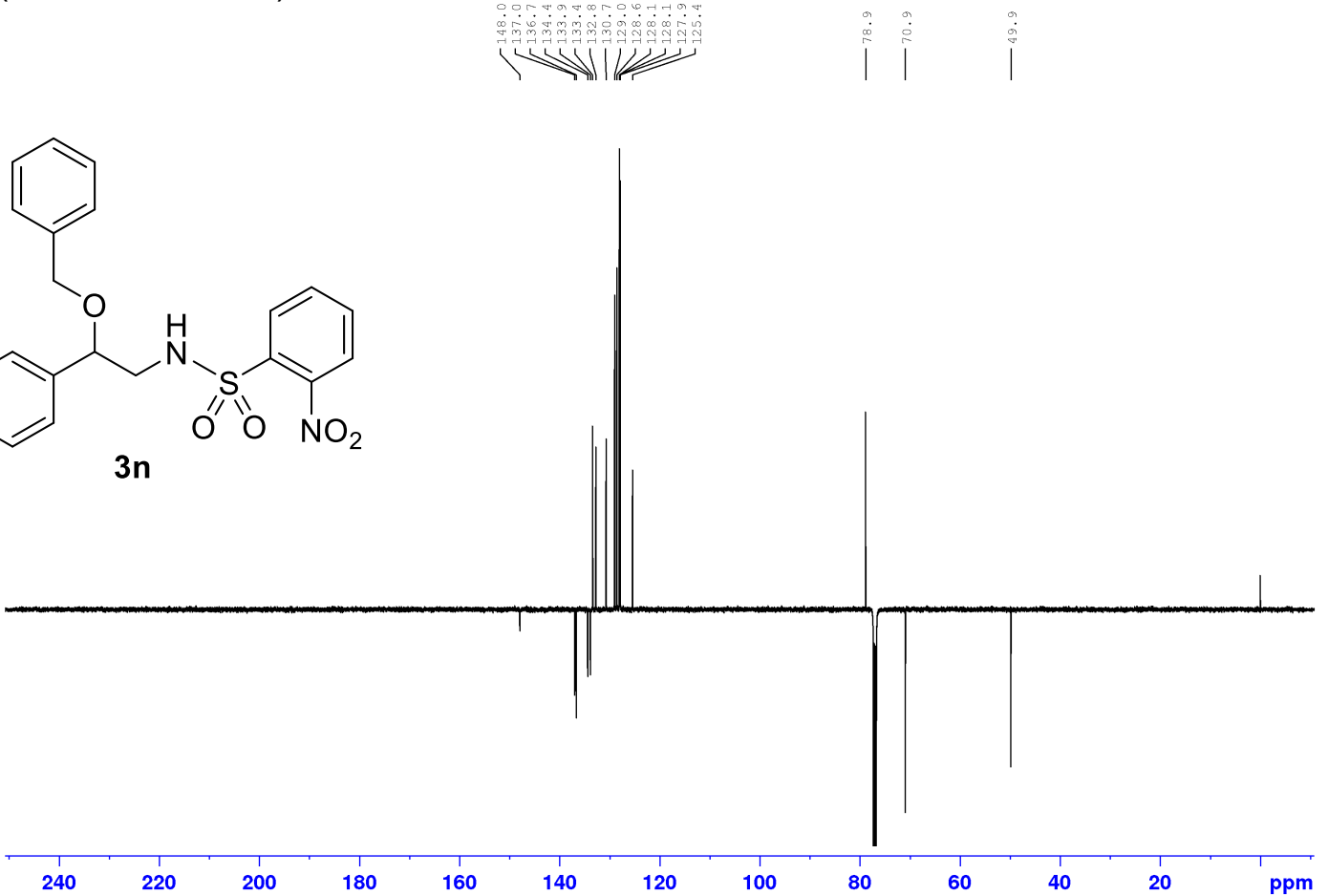
3o:

${ }^{1} \mathrm{H}\left(\mathrm{CDCl}_{3}, 500 \mathrm{MHz}\right)$

VIVIV Vil/

\|

$\left.\right|_{30} ^{\mathrm{Cl}}$

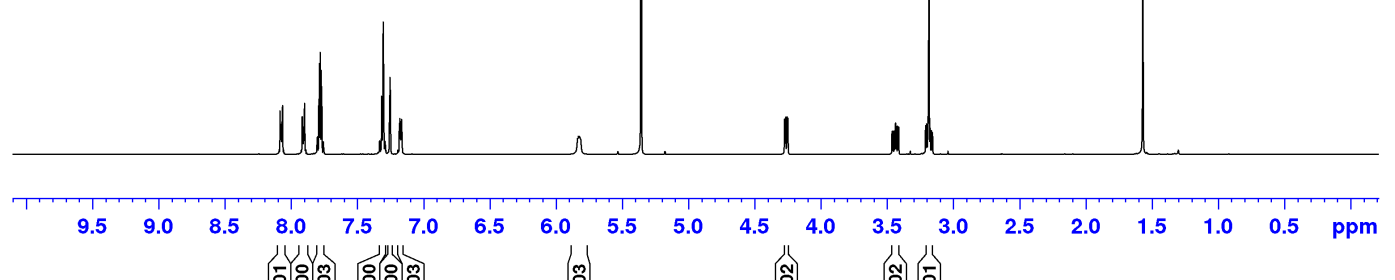

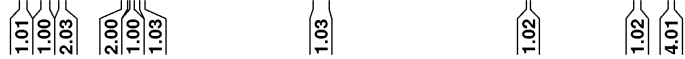

${ }^{13} \mathrm{C}\left(\mathrm{CDCl}_{3}, 125 \mathrm{MHz}\right)$<smiles>COC(CNS(=O)(=O)c1ccccc1[N+](=O)[O-])c1cccc(Cl)c1</smiles>

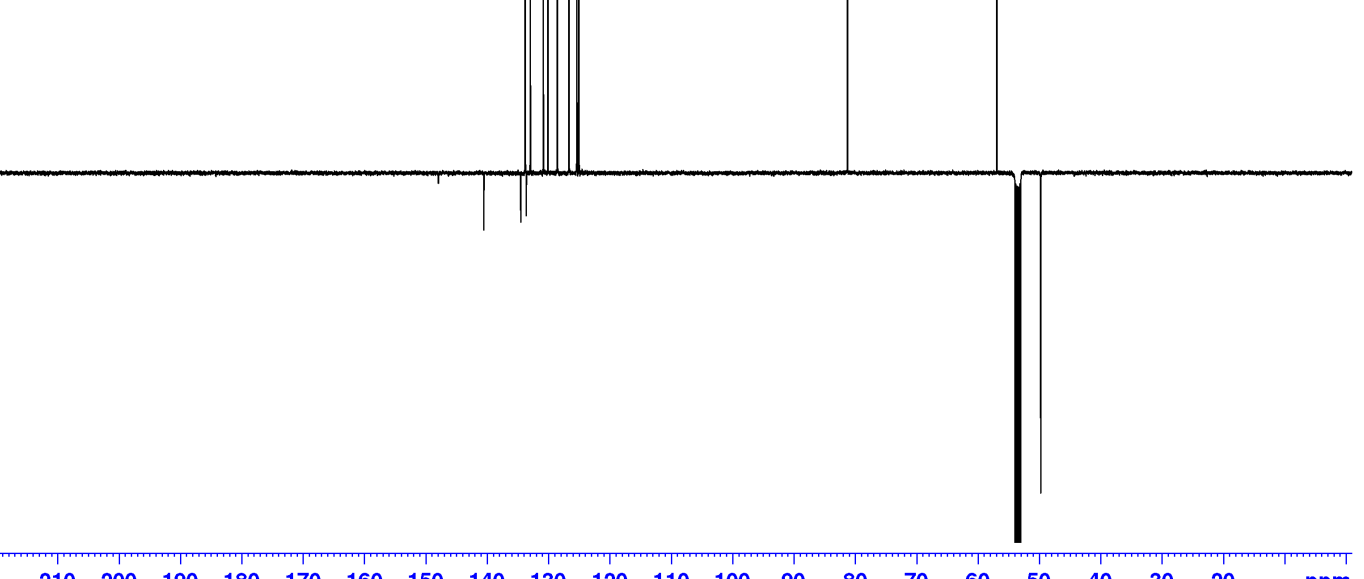


$3 p:$

${ }^{1} \mathrm{H}\left(\mathrm{CDCl}_{3}, 500 \mathrm{MHz}\right)$

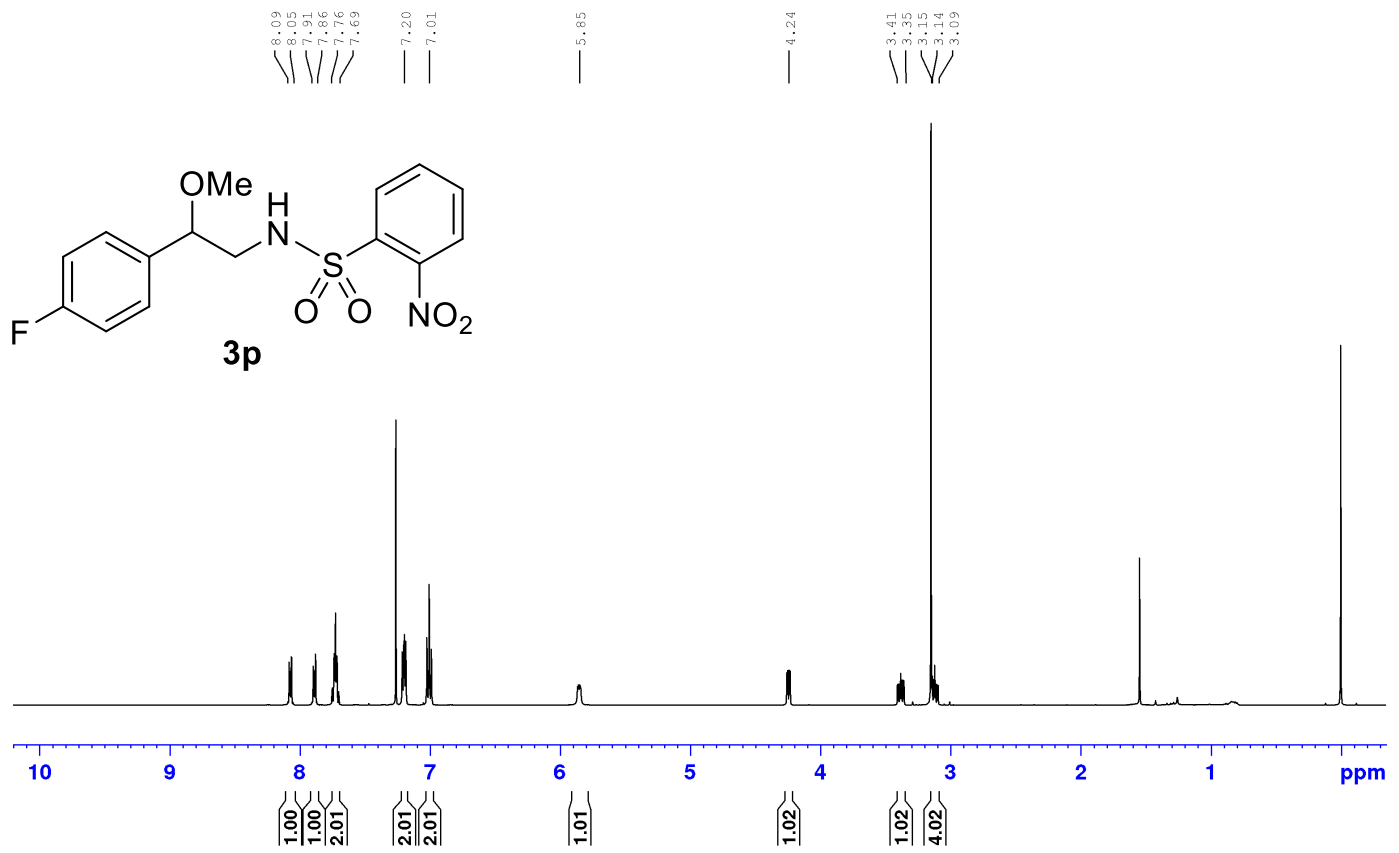

${ }^{13} \mathrm{C}\left(\mathrm{CDCl}_{3}, 125 \mathrm{MHz}\right)$

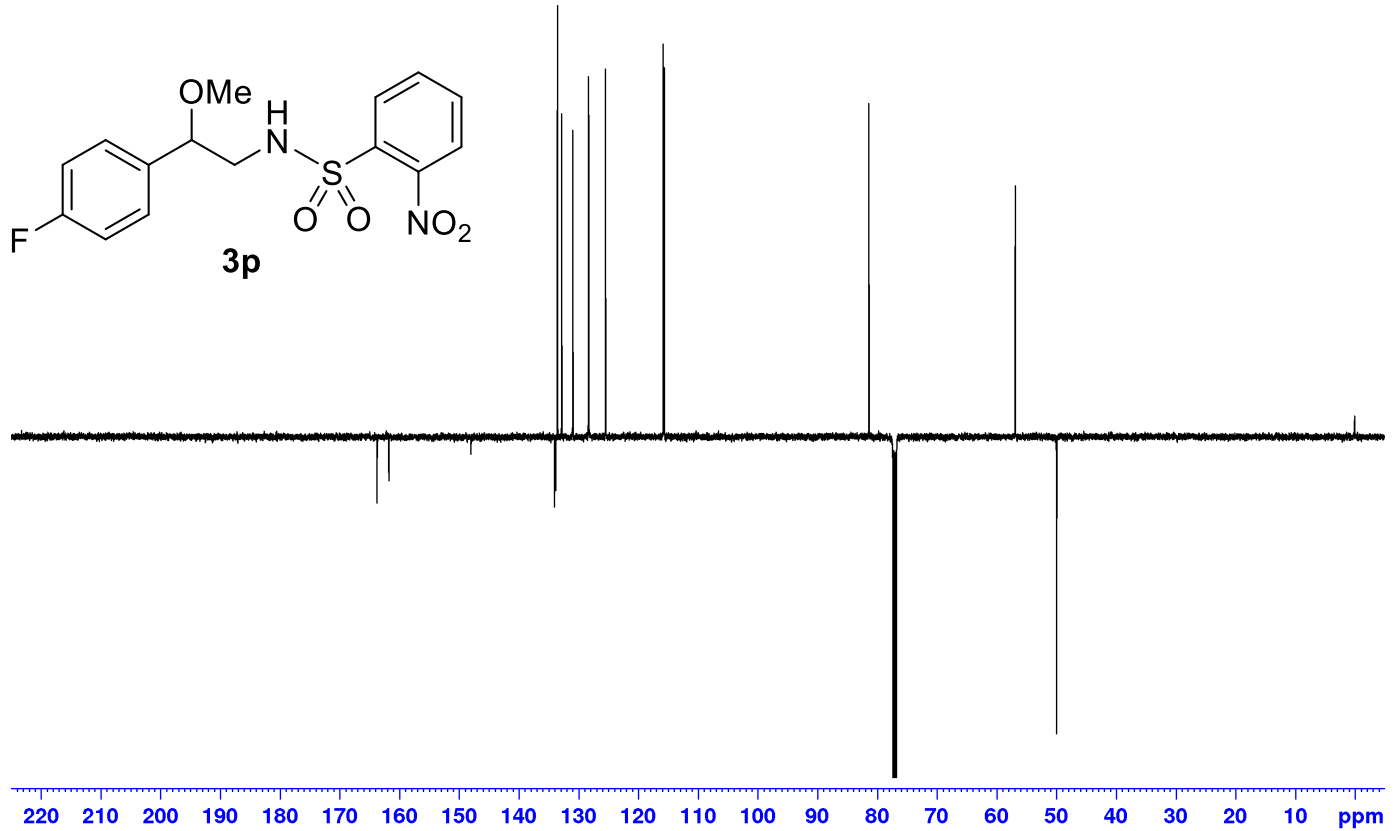


$3 q:$

${ }^{1} \mathrm{H}\left(\mathrm{CDCl}_{3}, 500 \mathrm{MHz}\right)$

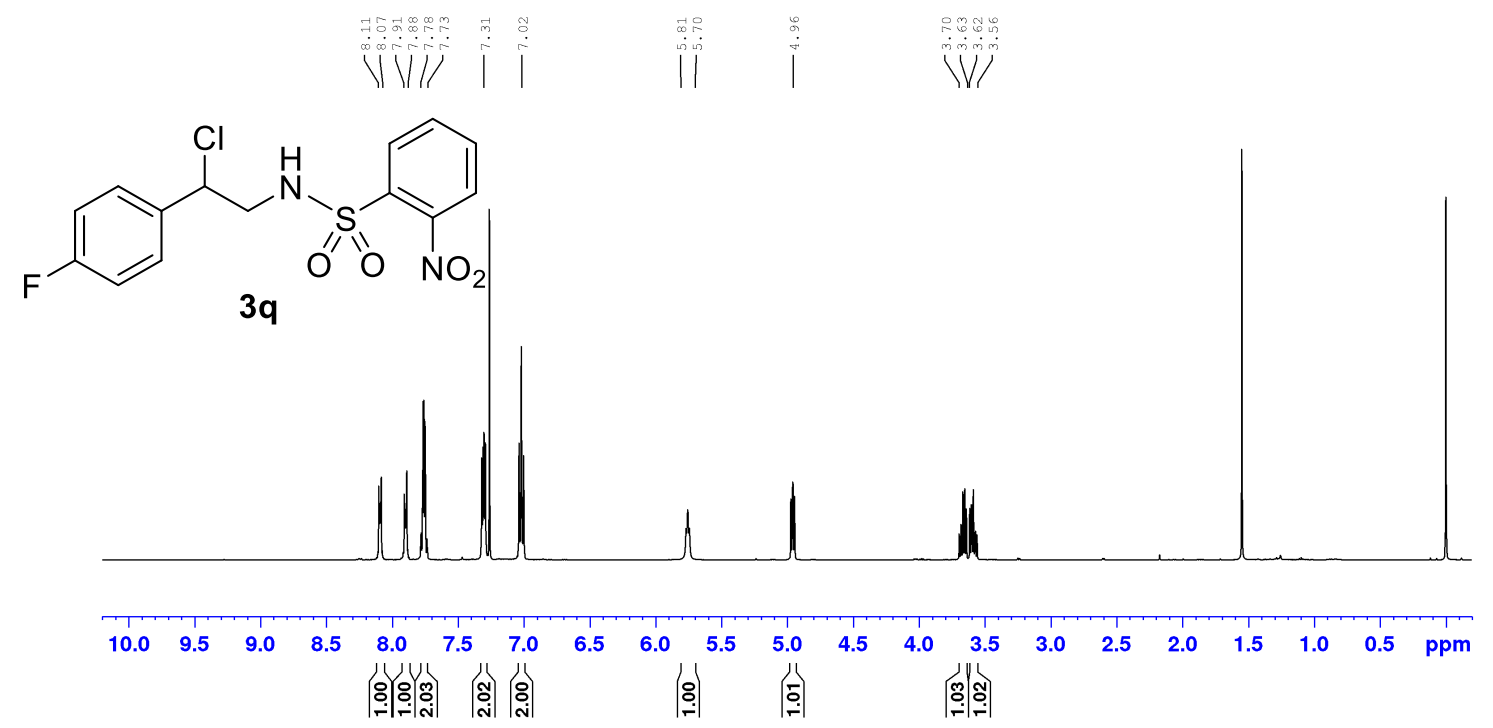

${ }^{13} \mathrm{C}\left(\mathrm{CDCl}_{3}, 125 \mathrm{MHz}\right)$

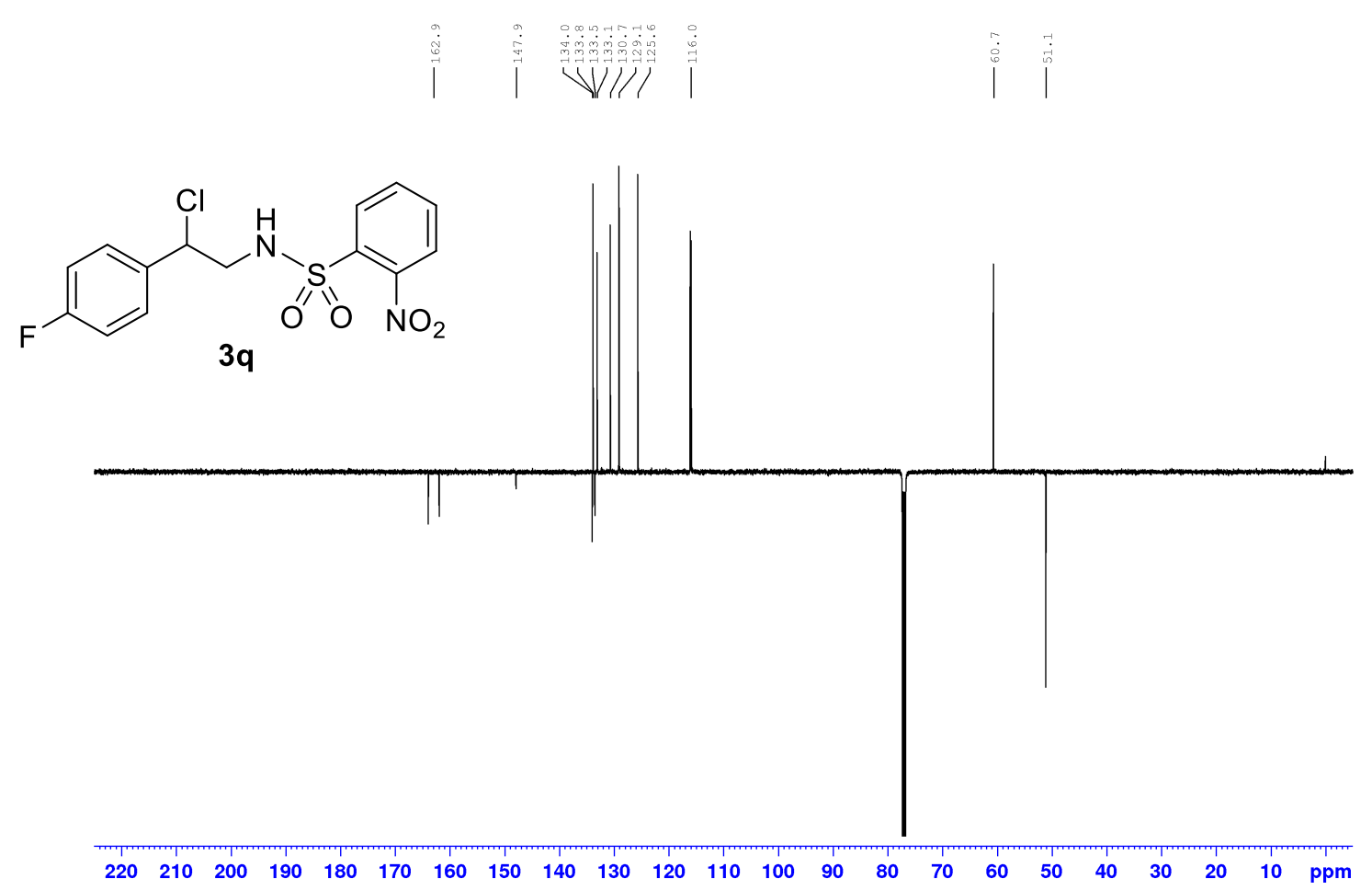


3r:

${ }^{1} \mathrm{H}\left(\mathrm{CDCl}_{3}, 500 \mathrm{MHz}\right)$

ปั<smiles>CCOC(CNS(=O)(=O)c1ccc(C)cn1)c1ccc(F)cc1</smiles>

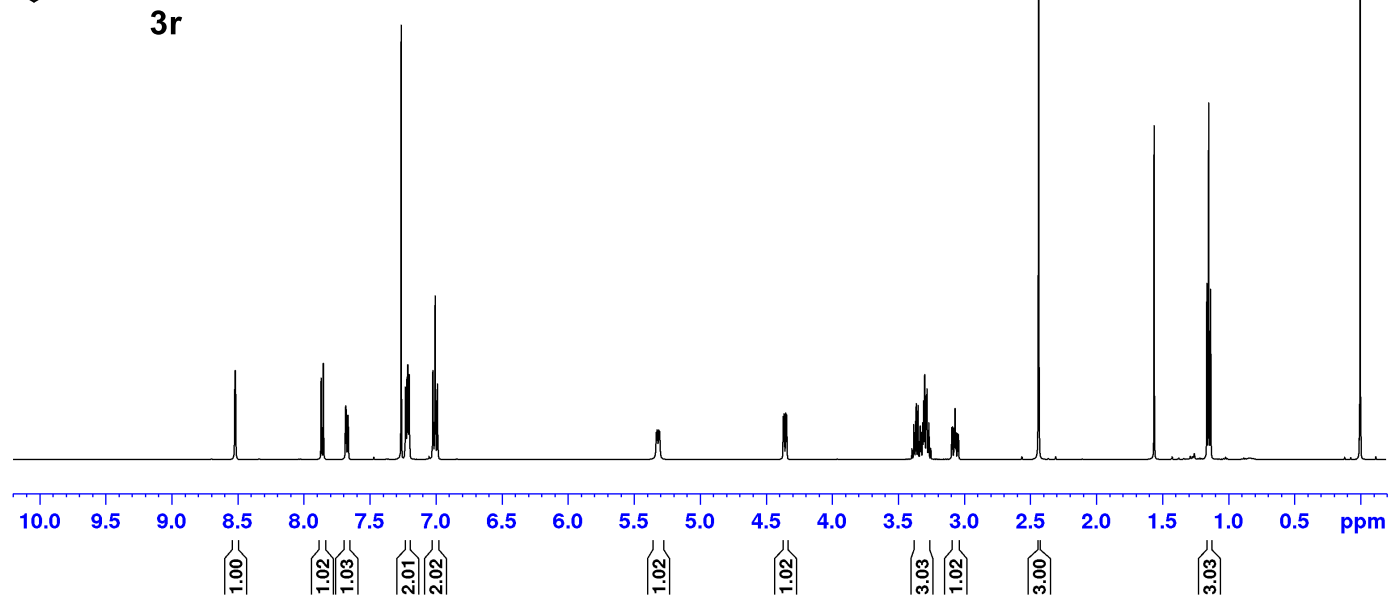

${ }^{13} \mathrm{C}\left(\mathrm{CDCl}_{3}, 125 \mathrm{MHz}\right)$

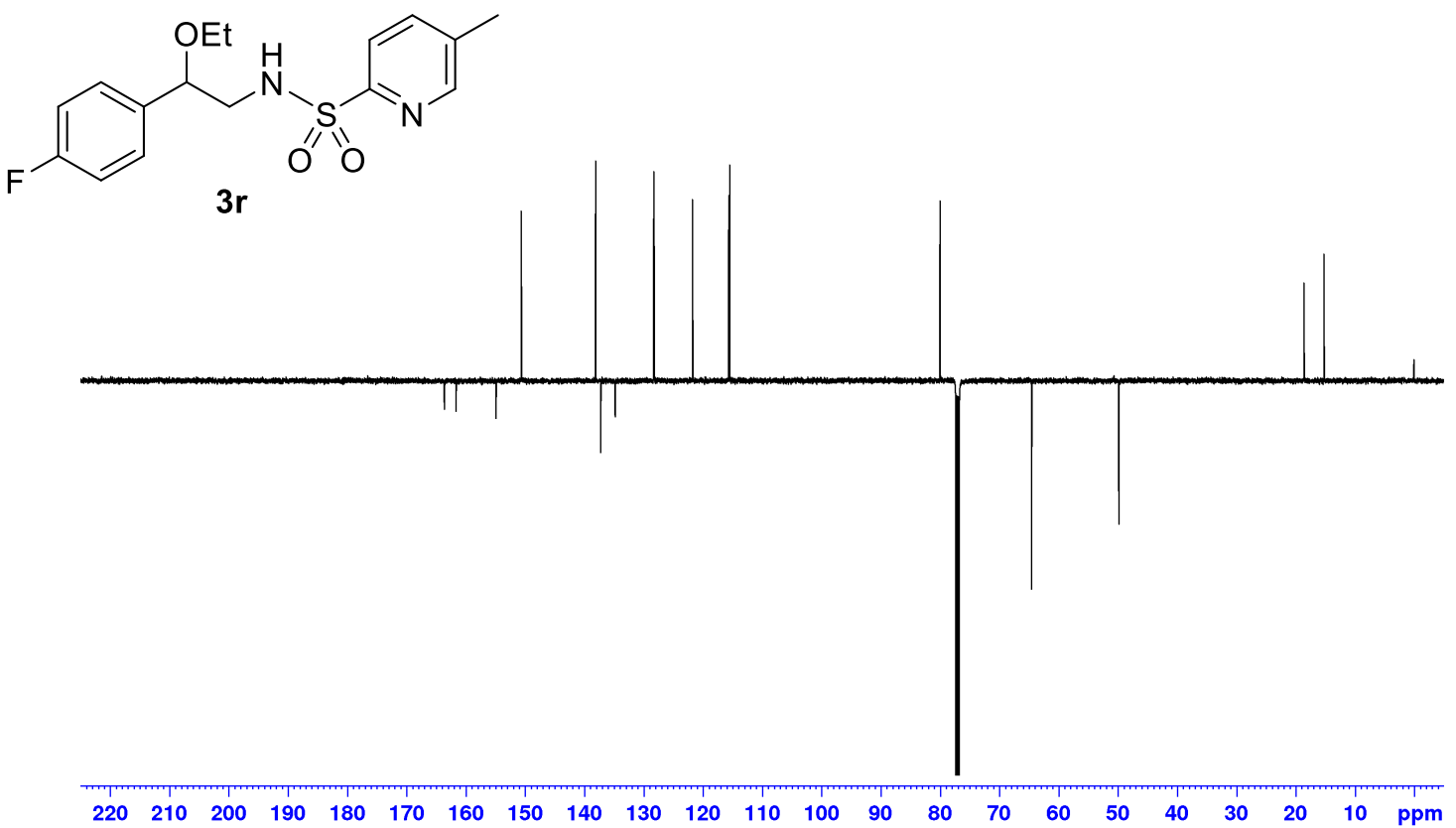


The regioselectivity of ring openings confirmed by HMBC (key correlations indicated in box):

HMBC for $3 a$ :

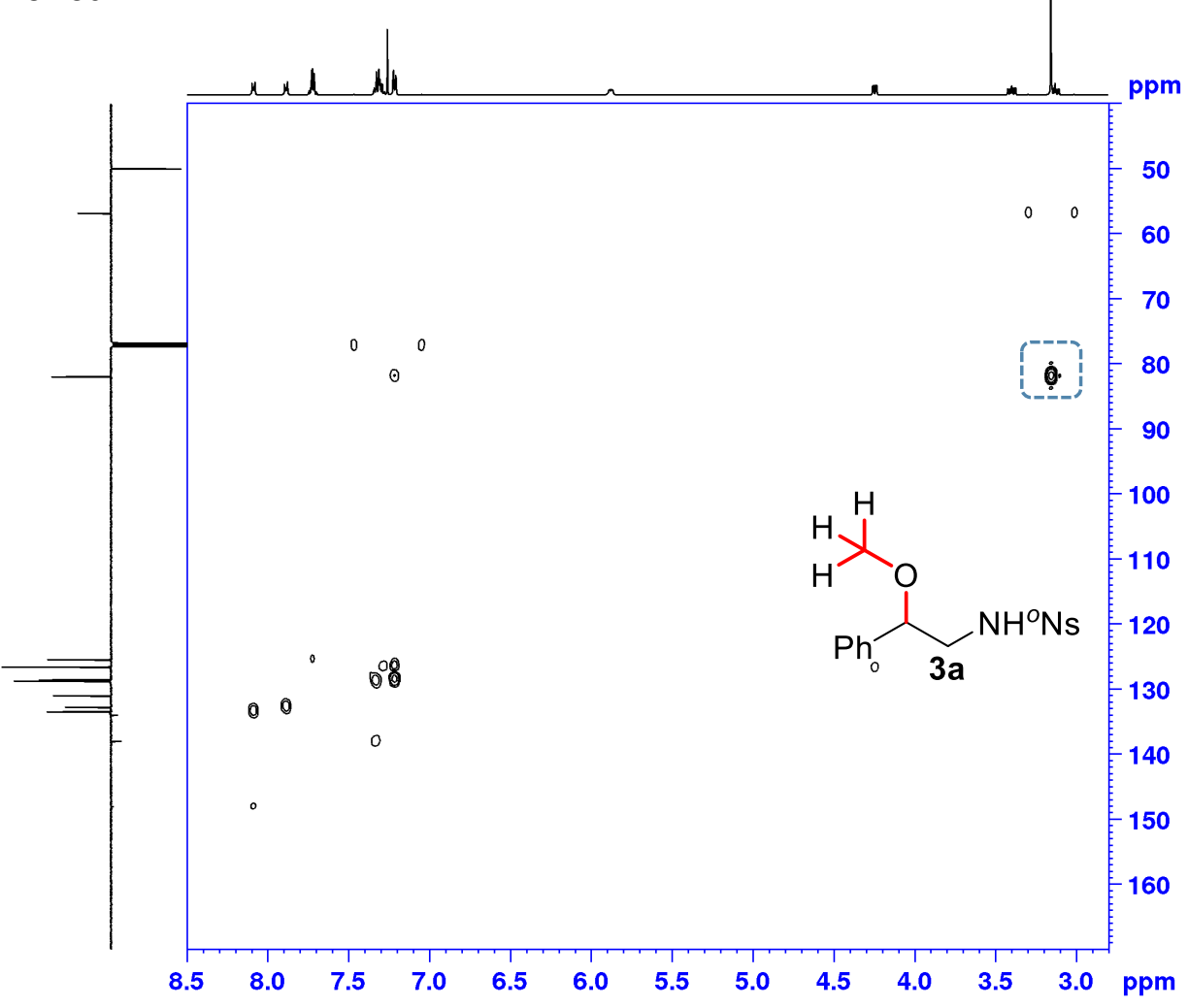

$\mathrm{HMBC}$ for $\mathbf{3 b}$ :

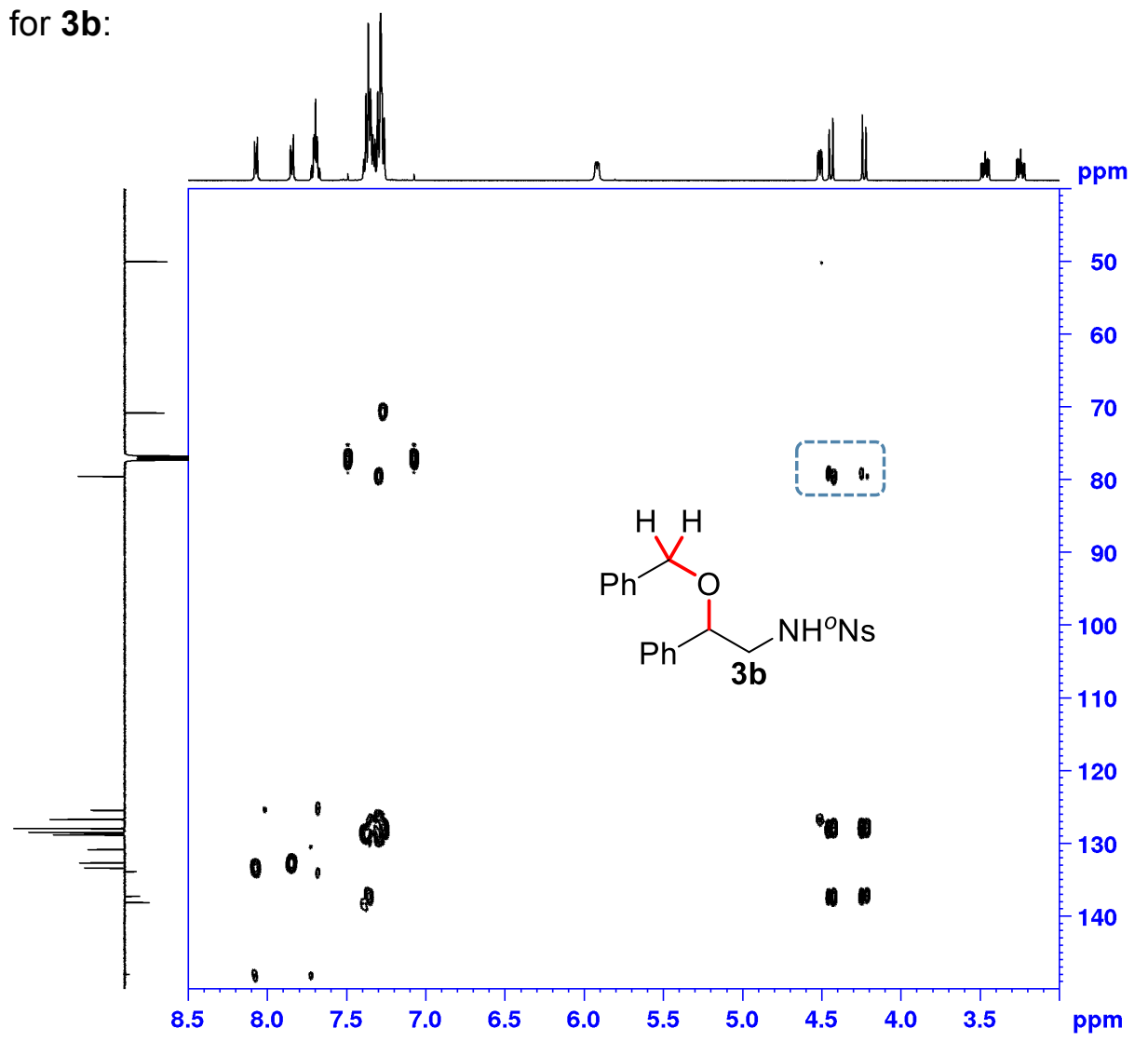


HMBC for $\mathbf{3 d :}$

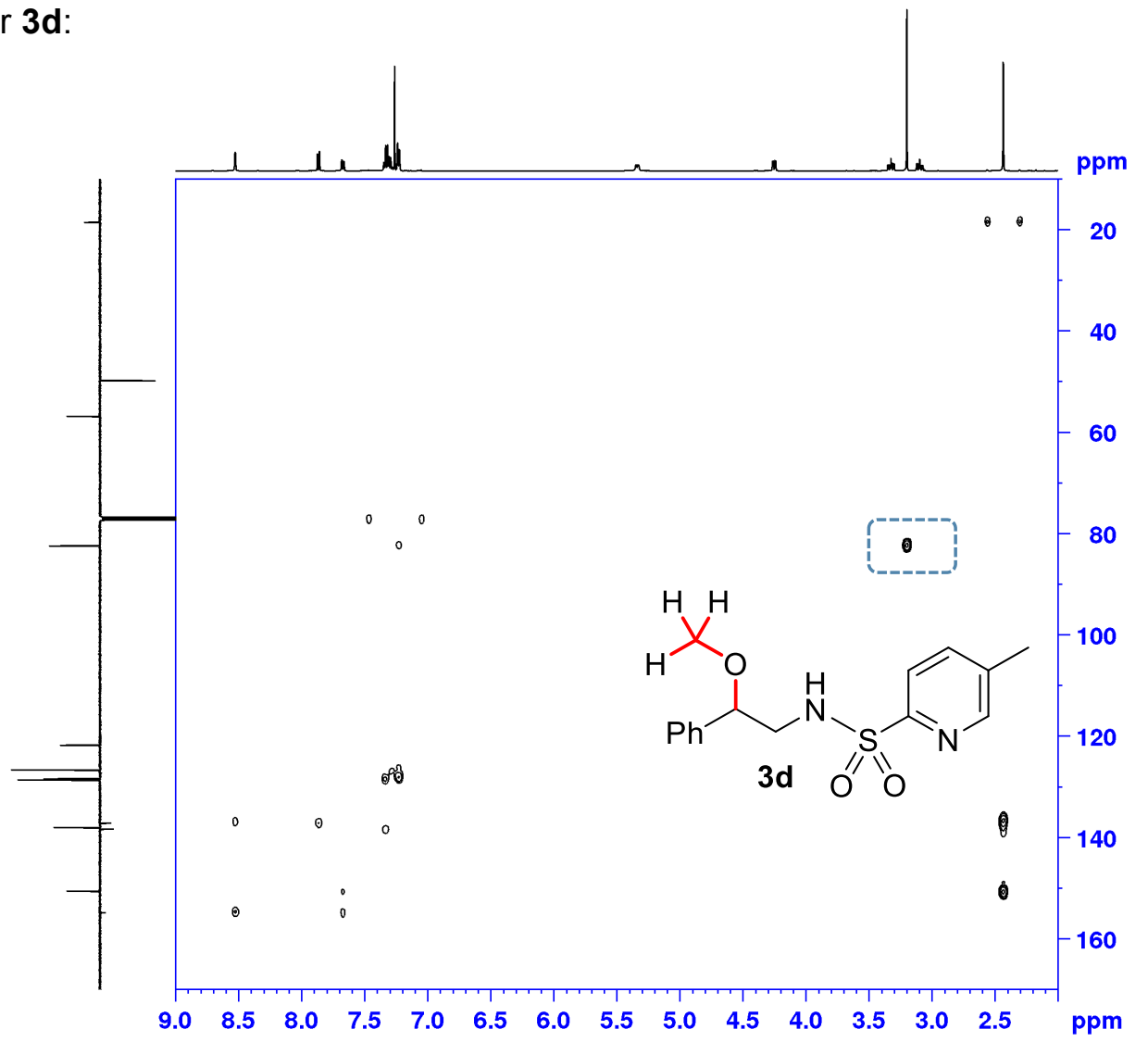

HMBC for $\mathbf{3 e}$ :

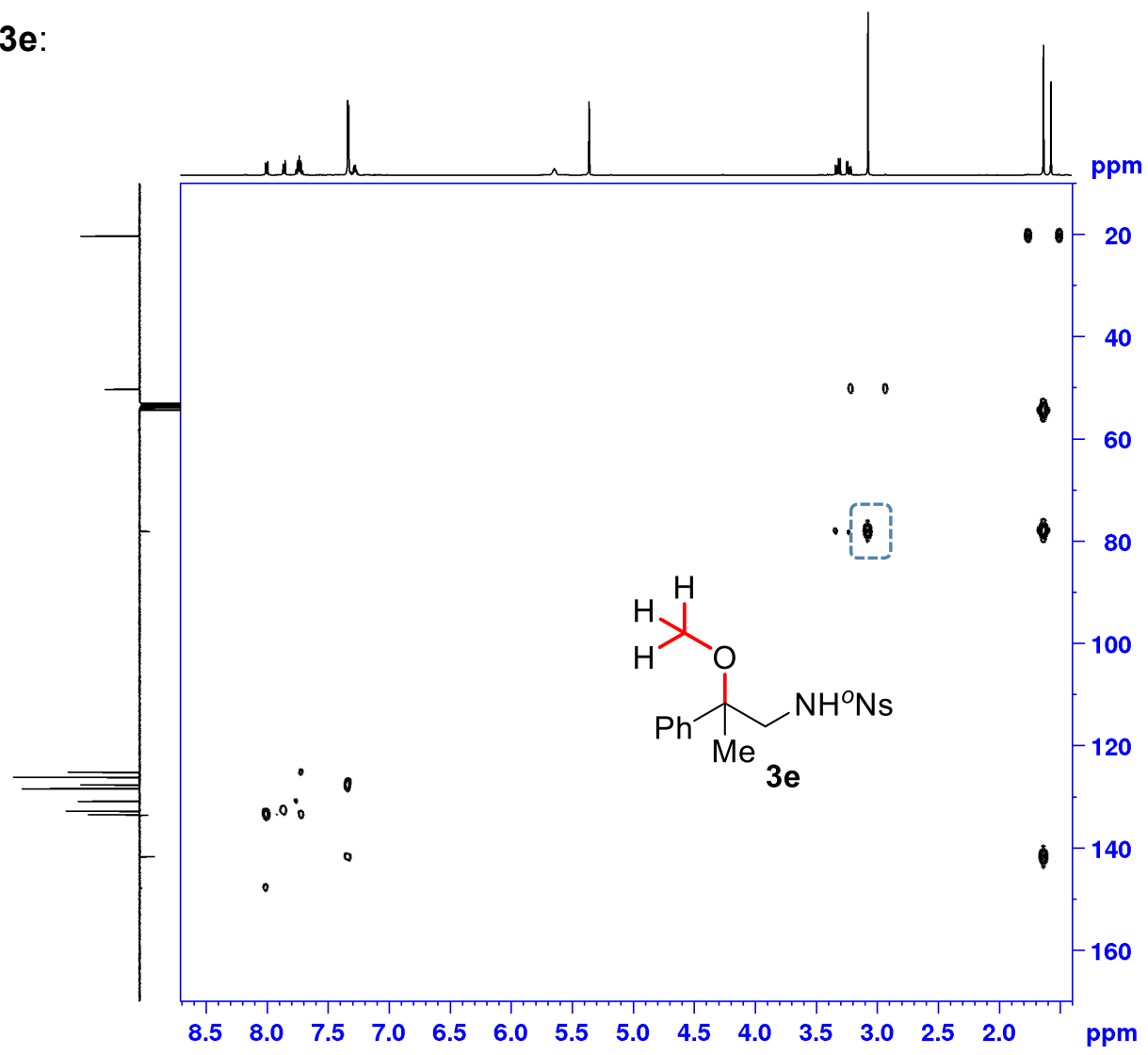


HMBC for 3f:

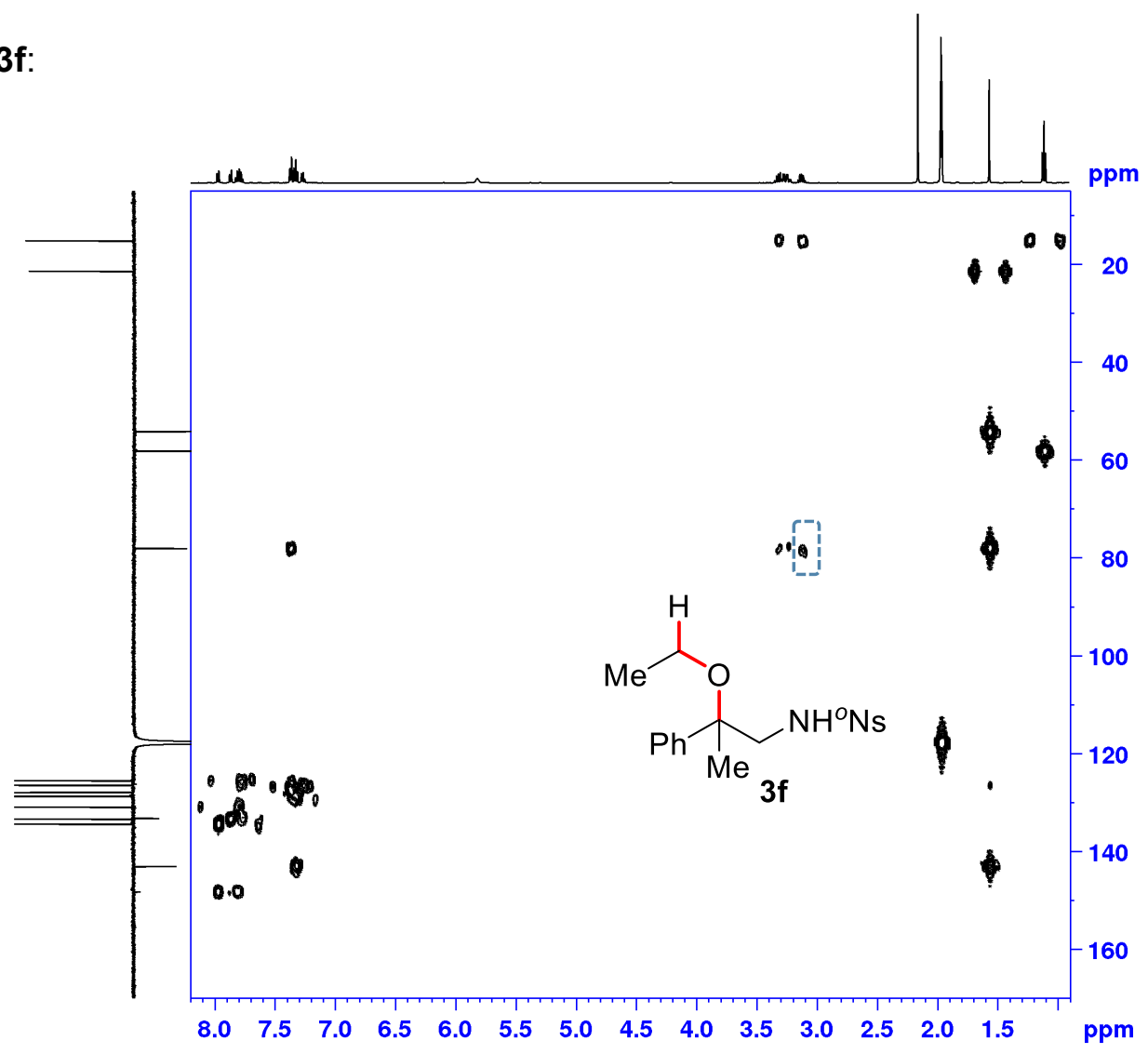

HMBC for $\mathbf{3 g}$ :

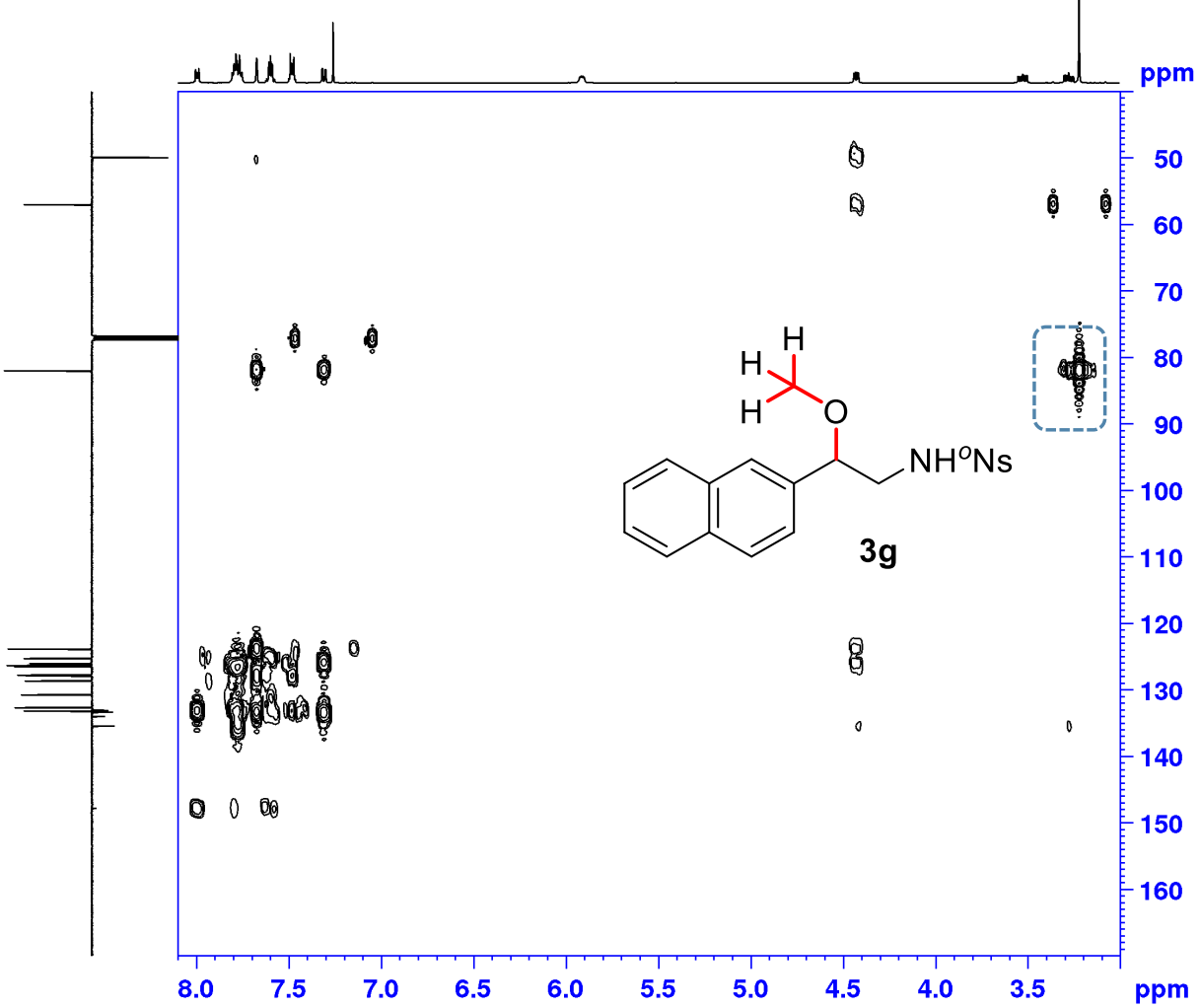


HMBC for $\mathbf{3} \mathbf{i}$ :

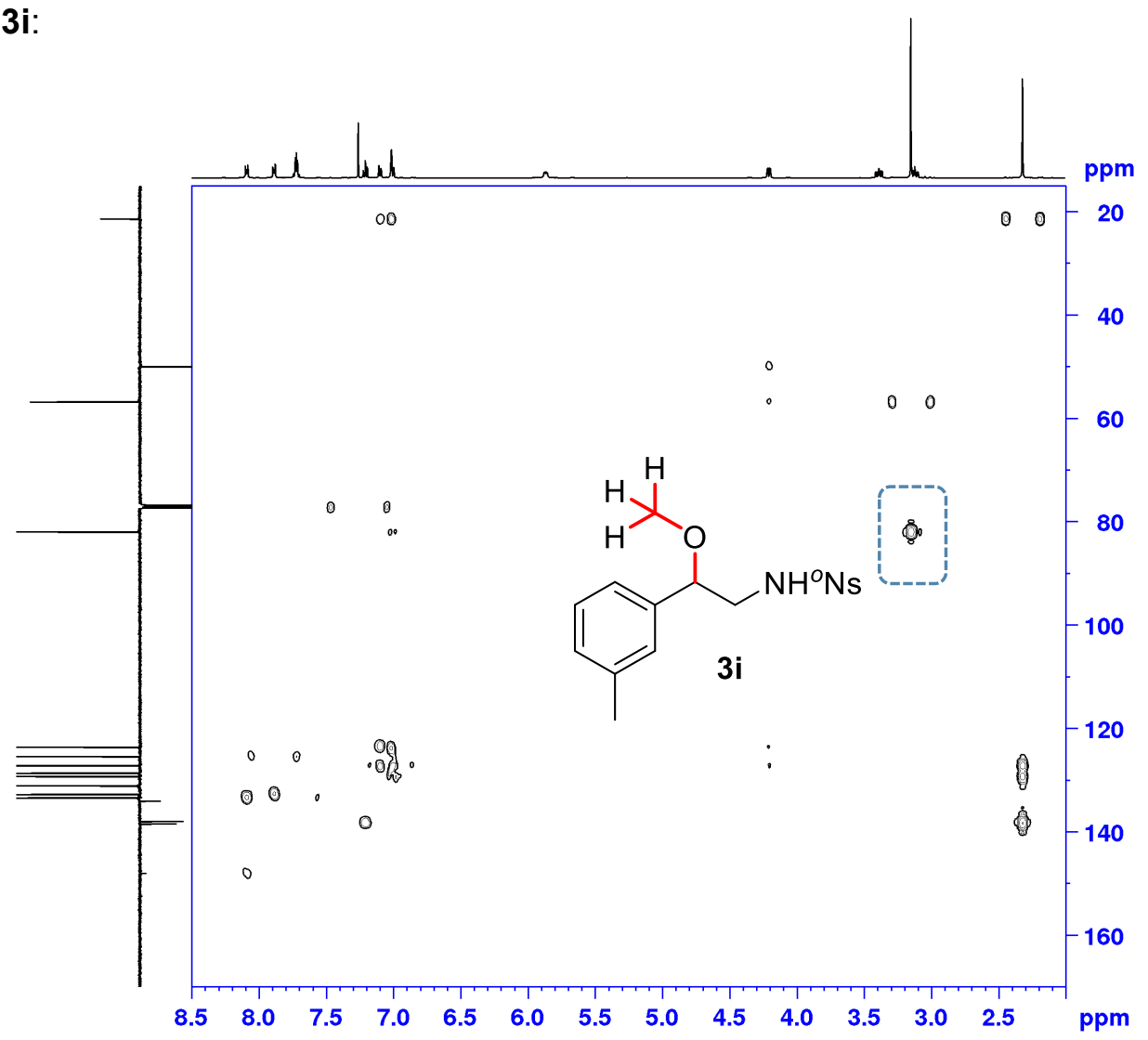

$\mathrm{HMBC}$ for $3 \mathrm{j}$

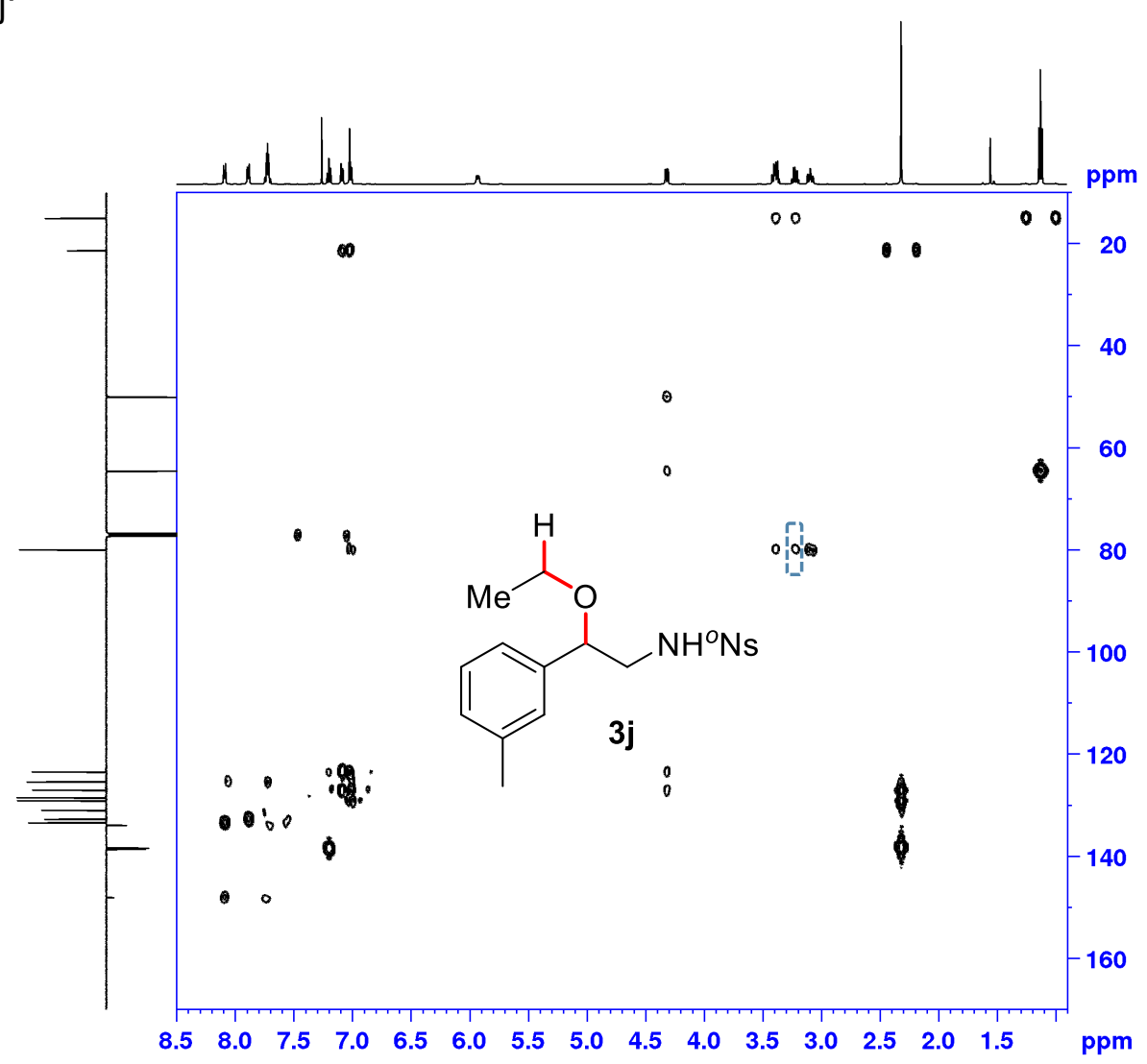


HMBC for $\mathbf{3 k}$ :

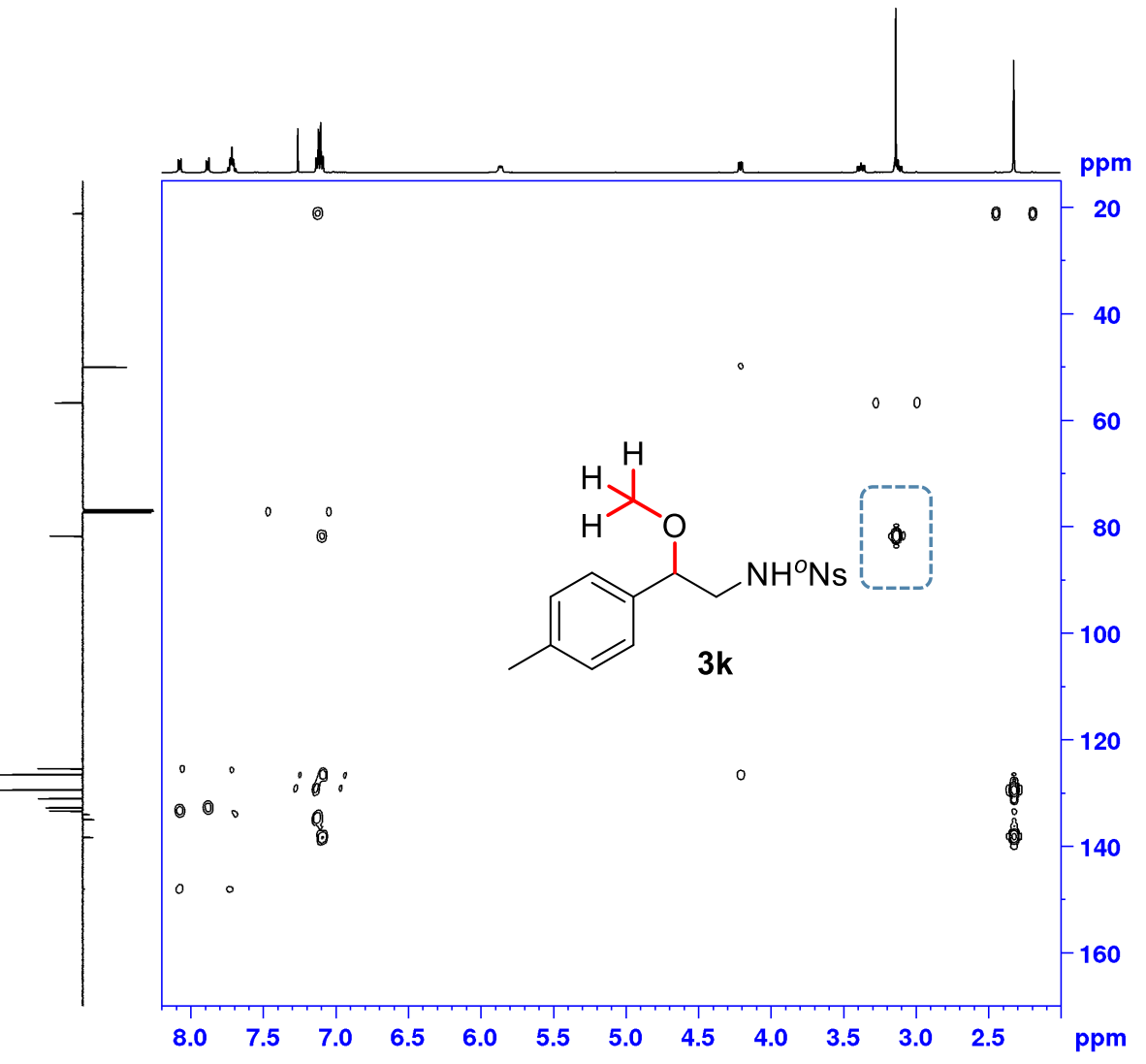

HMBC for $\mathbf{3 m}$ :

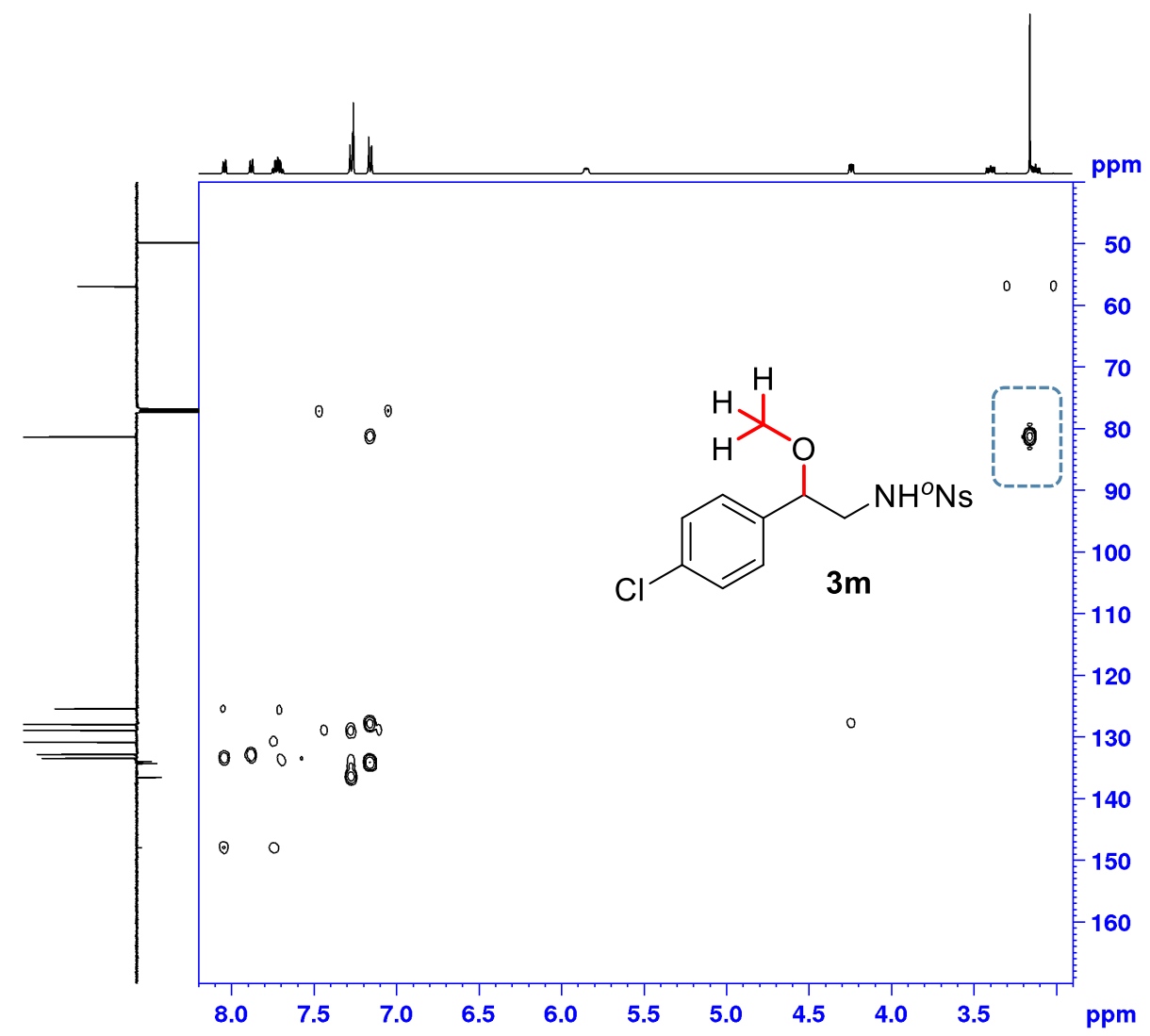


HMBC for $\mathbf{3 n}$ :

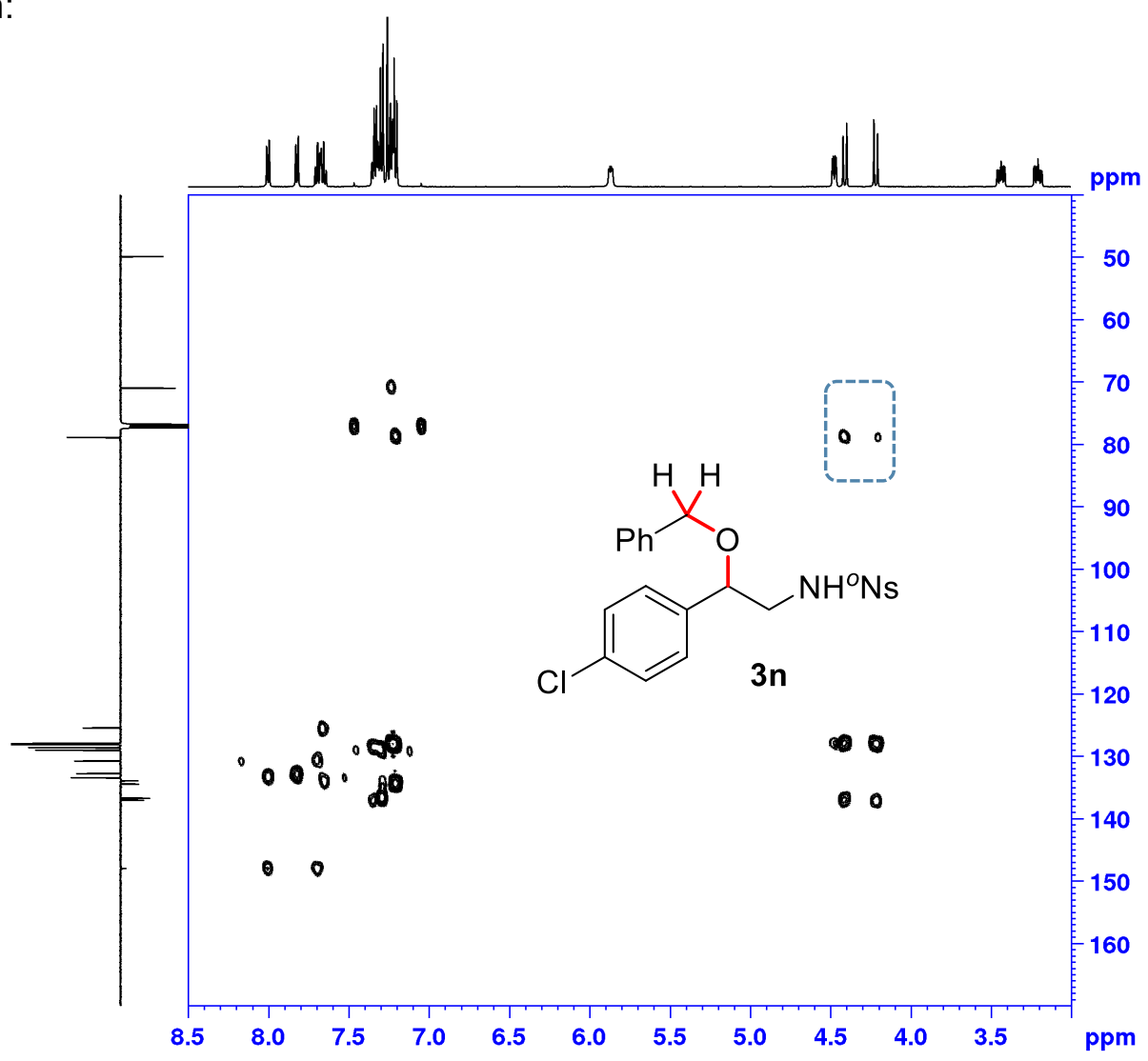

HMBC for 3o:

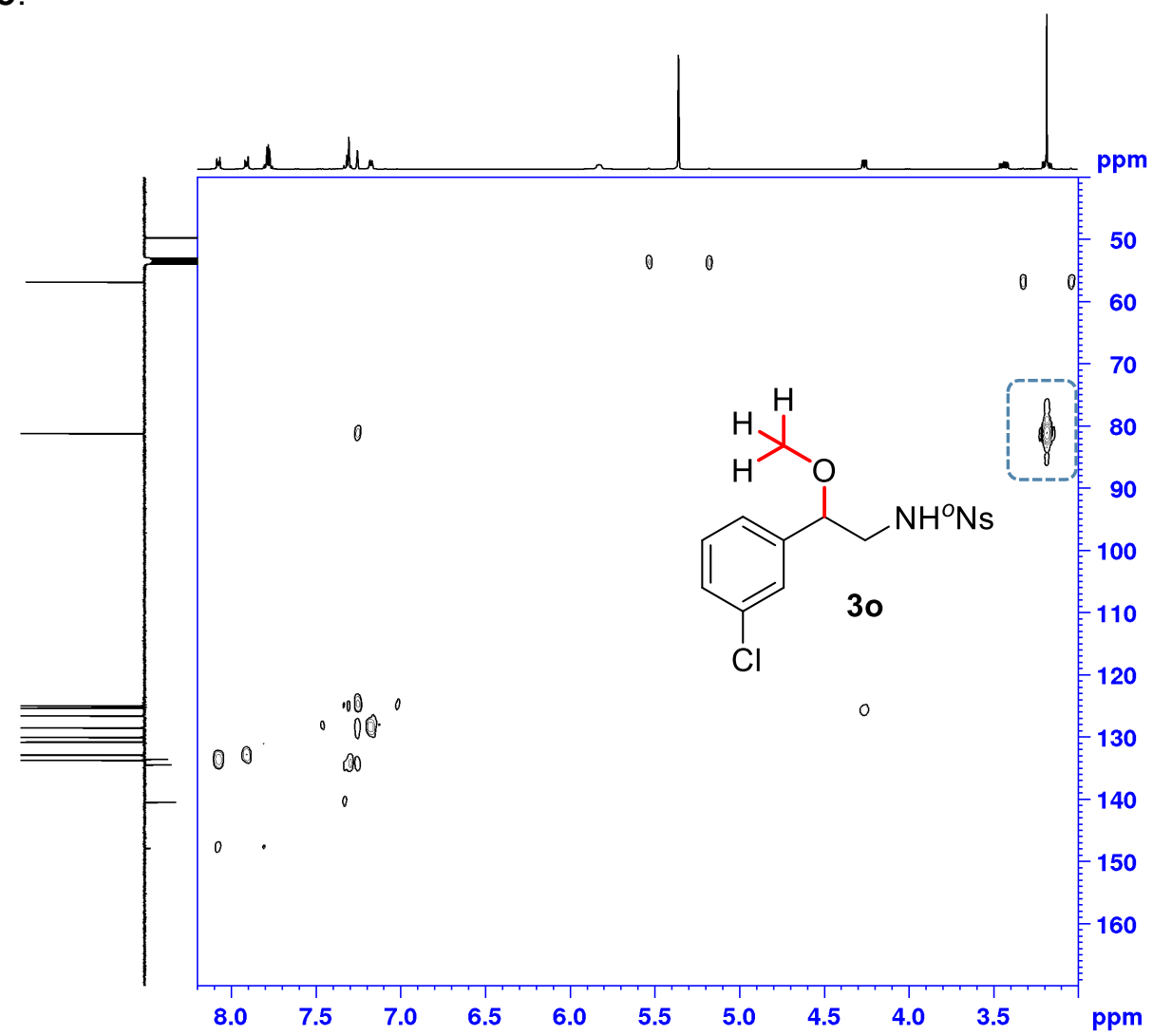


HMBC for $3 p$ :

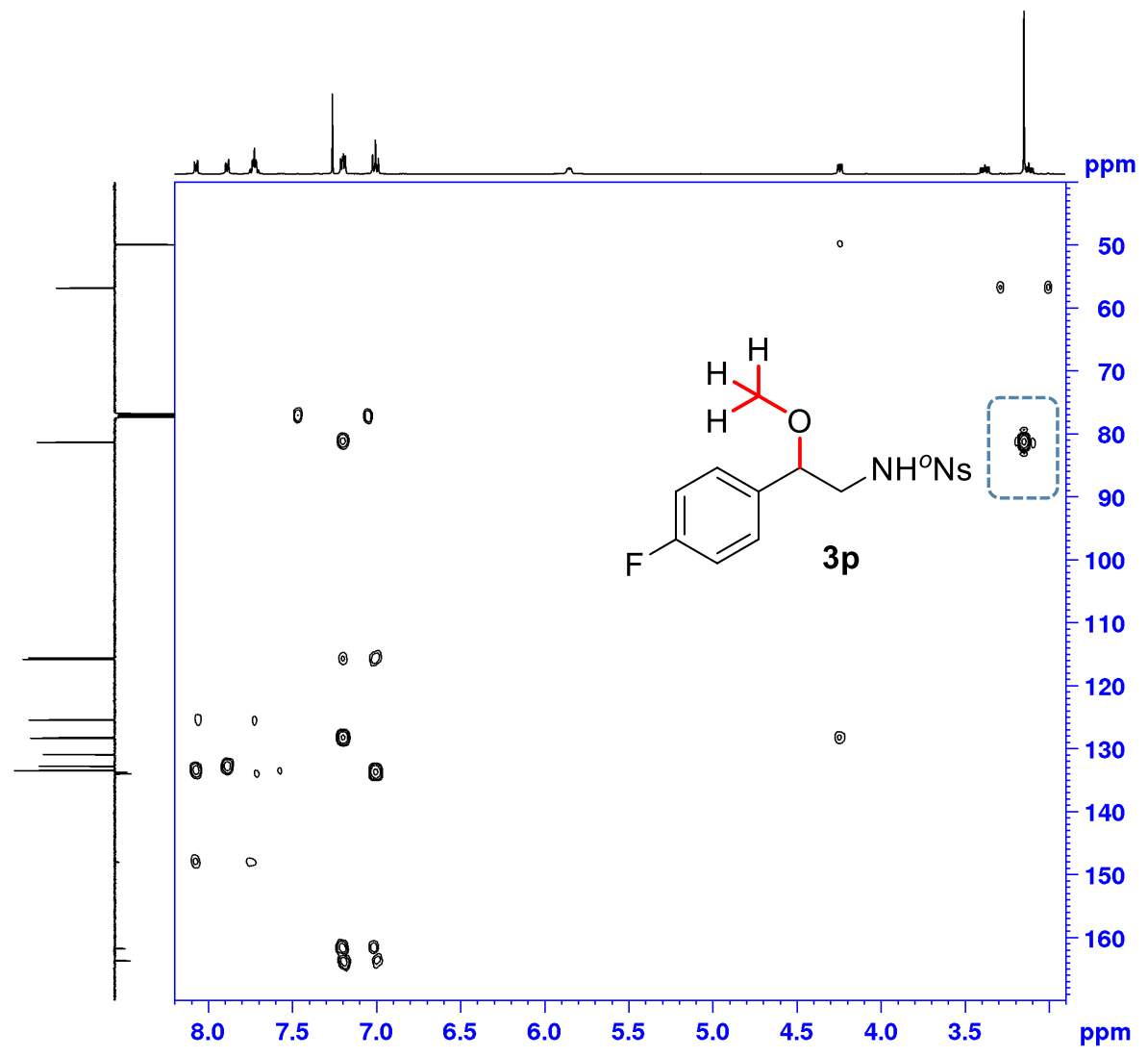

HMBC for $3 r$ :

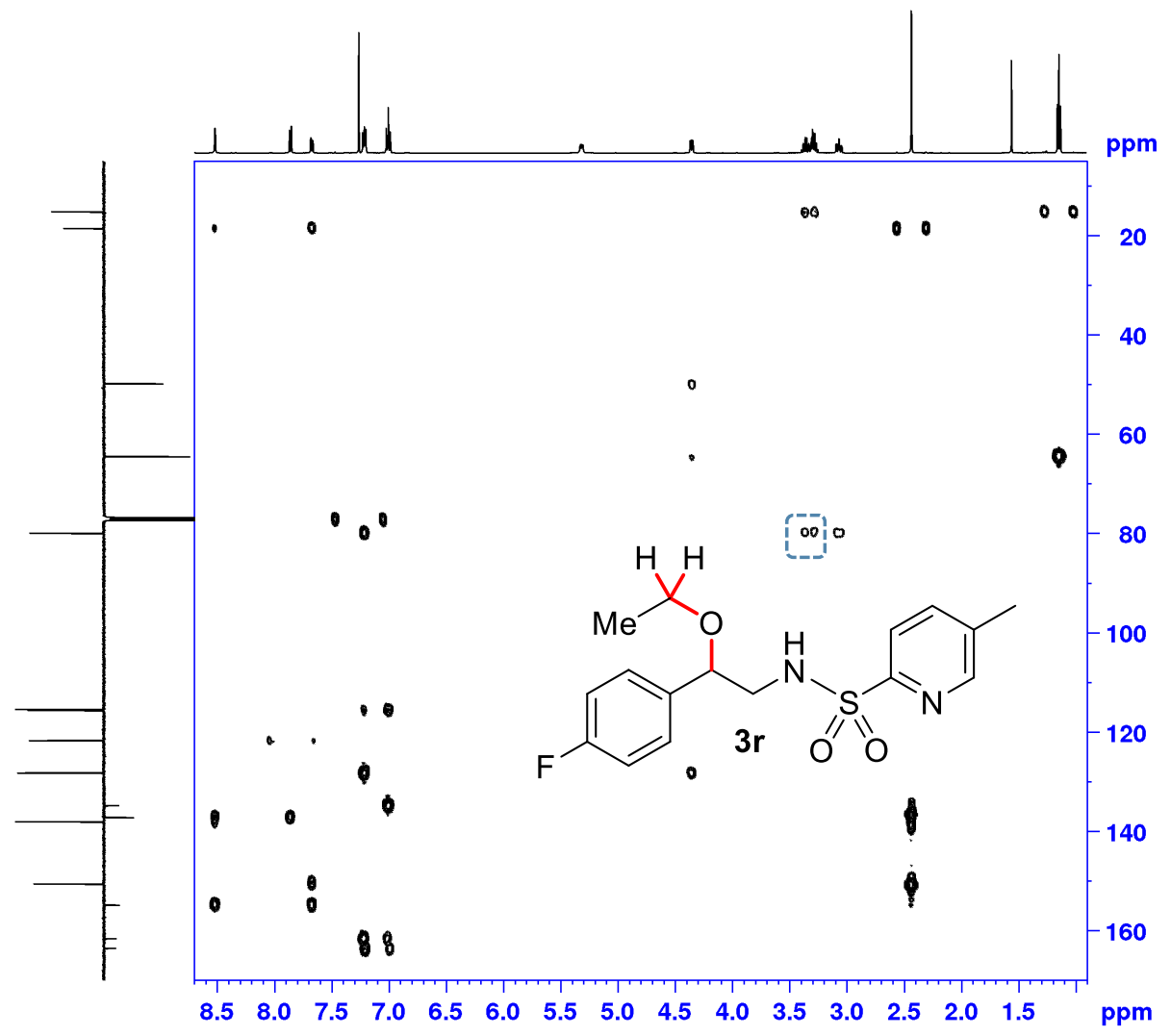


4a:

${ }^{1} \mathrm{H}\left(\mathrm{CDCl}_{3}, 500 \mathrm{MHz}\right)$

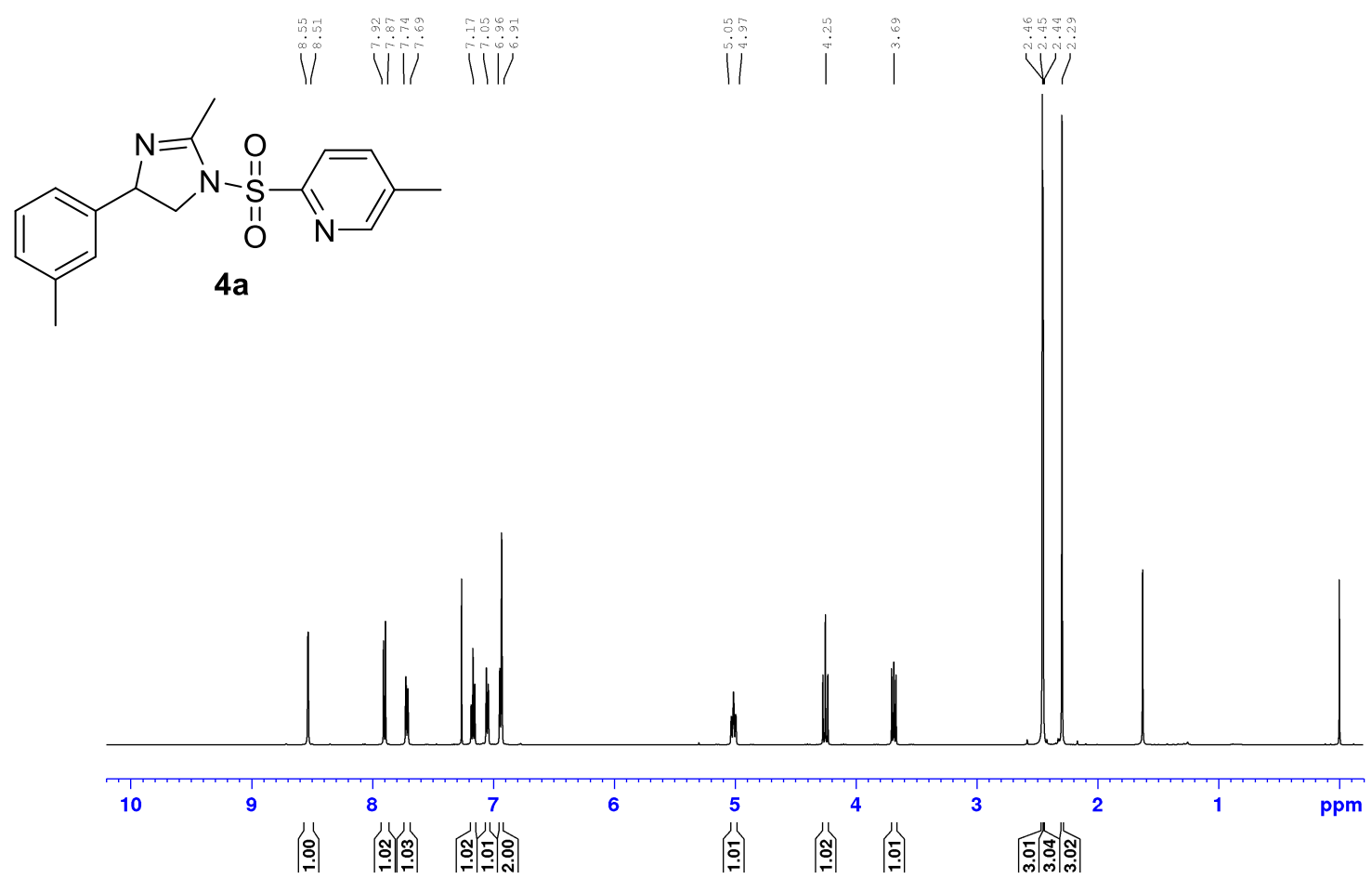

${ }^{13} \mathrm{C}\left(\mathrm{CDCl}_{3}, 125 \mathrm{MHz}\right)$

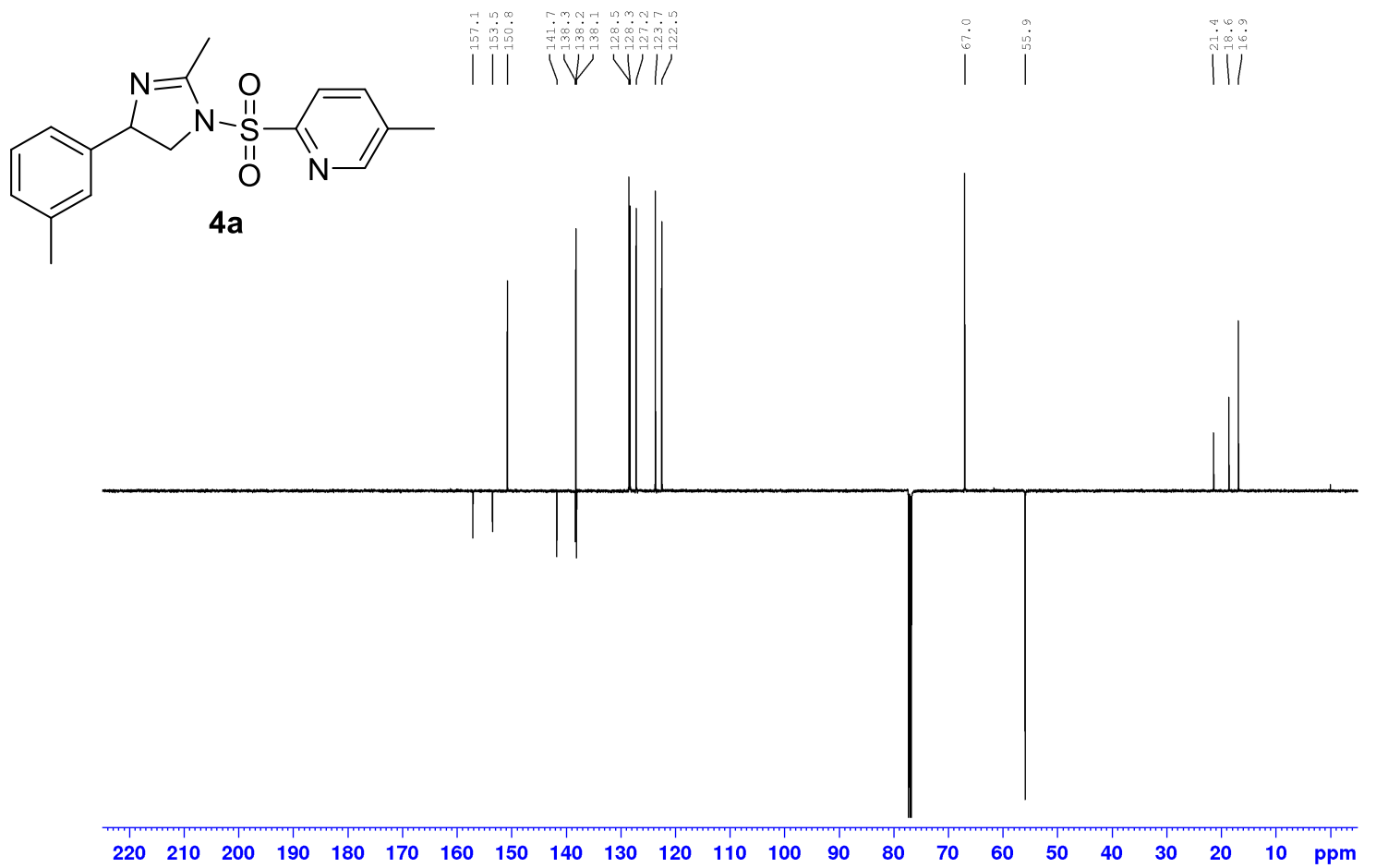


4b:

${ }^{1} \mathrm{H}\left(\mathrm{CDCl}_{3}, 500 \mathrm{MHz}\right)$

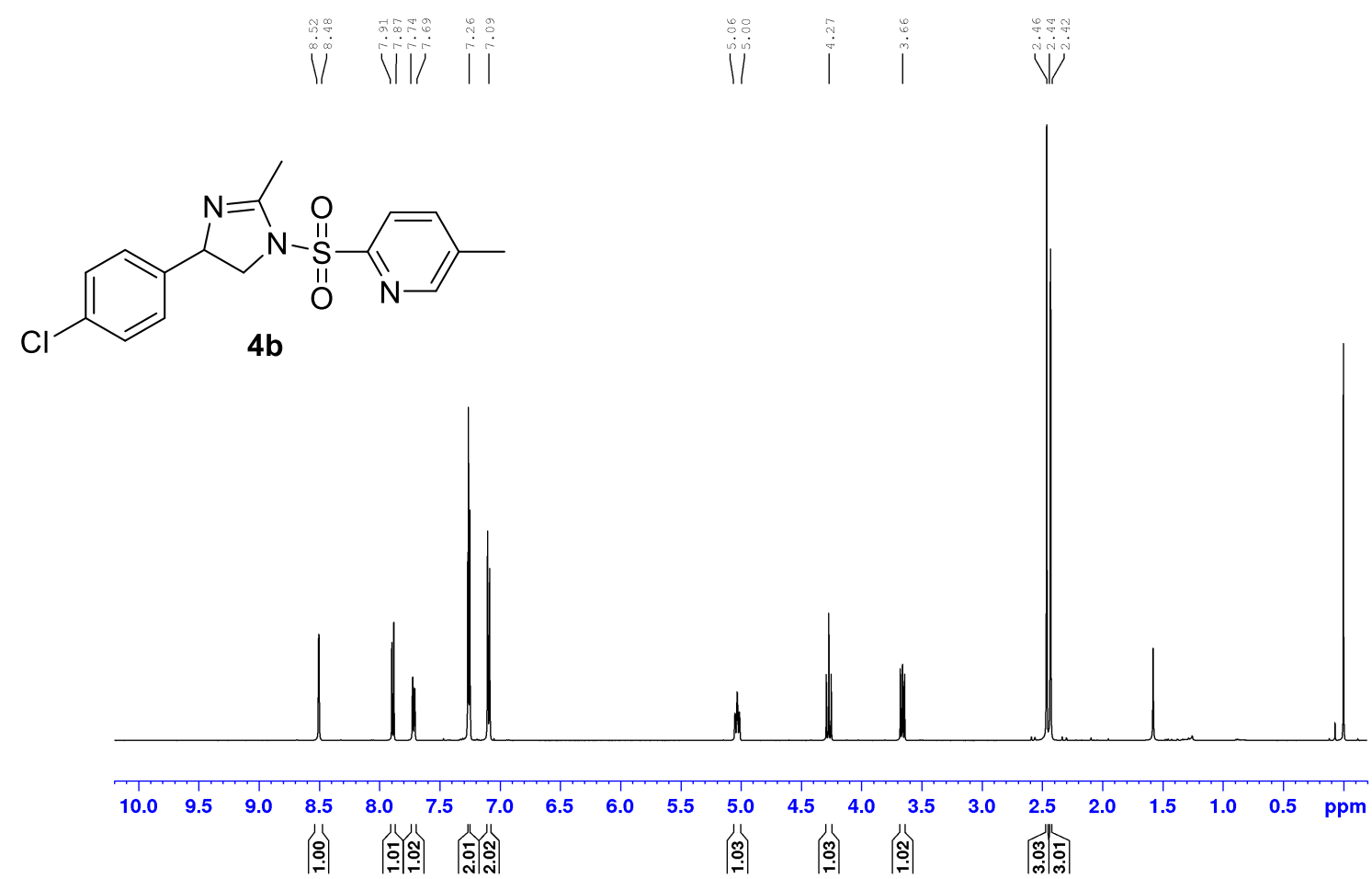

${ }^{13} \mathrm{C}\left(\mathrm{CDCl}_{3}, 125 \mathrm{MHz}\right)$

|l|<smiles>CC1=NC(c2ccc(Cl)cc2)CN1S(=O)(=O)c1ccc(C)cn1</smiles>

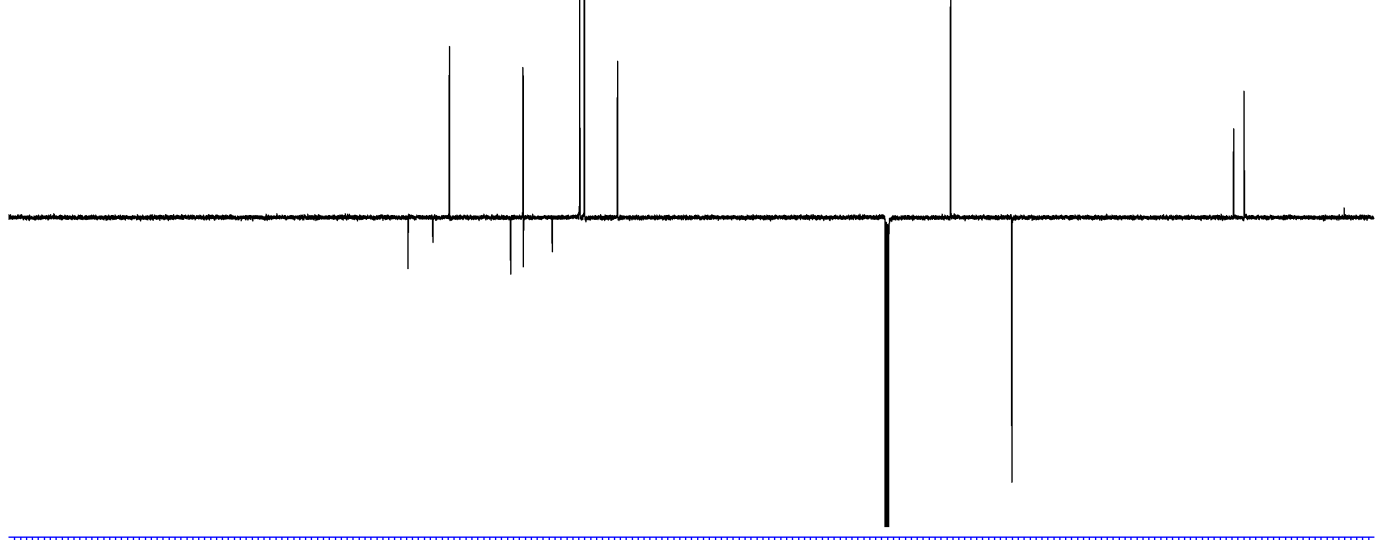

$\begin{array}{lllllllllllllllllllllll}220 & 210 & 200 & 190 & 180 & 170 & 160 & 150 & 140 & 130 & 120 & 110 & 100 & 90 & 80 & 70 & 60 & 50 & 40 & 30 & 20 & 10 & \mathrm{ppm}\end{array}$ 
4c:

${ }^{1} \mathrm{H}\left(\mathrm{CDCl}_{3}, 500 \mathrm{MHz}\right)$

II

||

Vั.

$\underbrace{N=S C}_{4 c}$

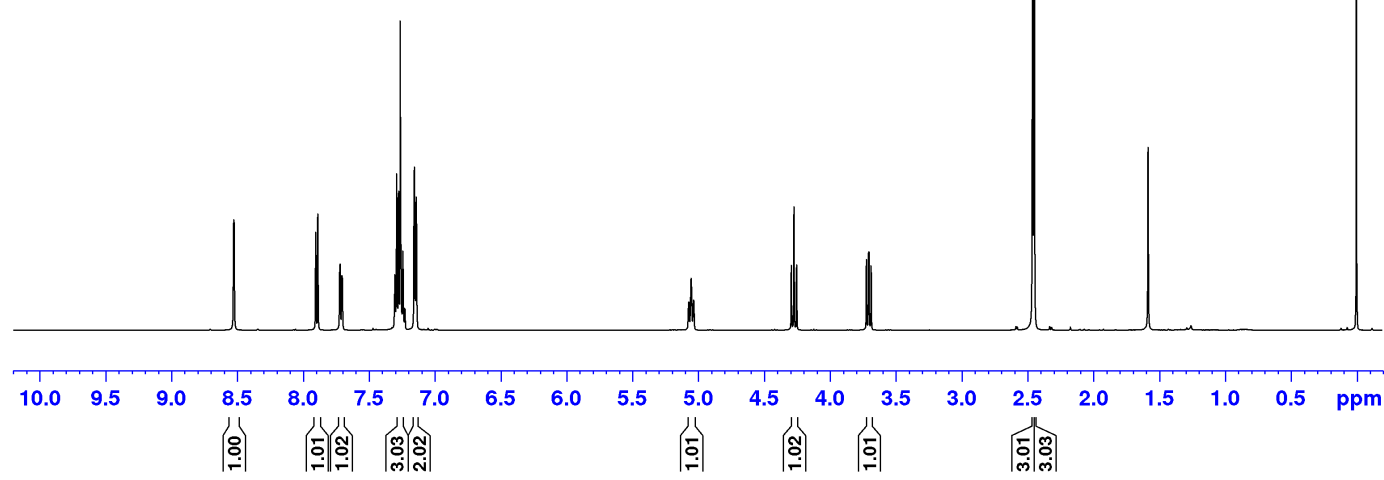

${ }^{13} \mathrm{C}\left(\mathrm{CDCl}_{3}, 125 \mathrm{MHz}\right)$

l|

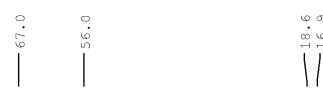

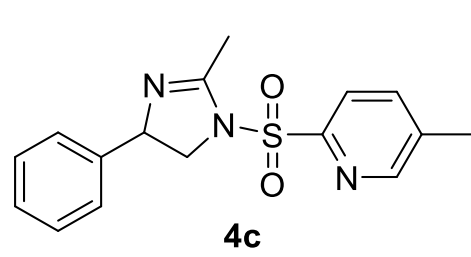

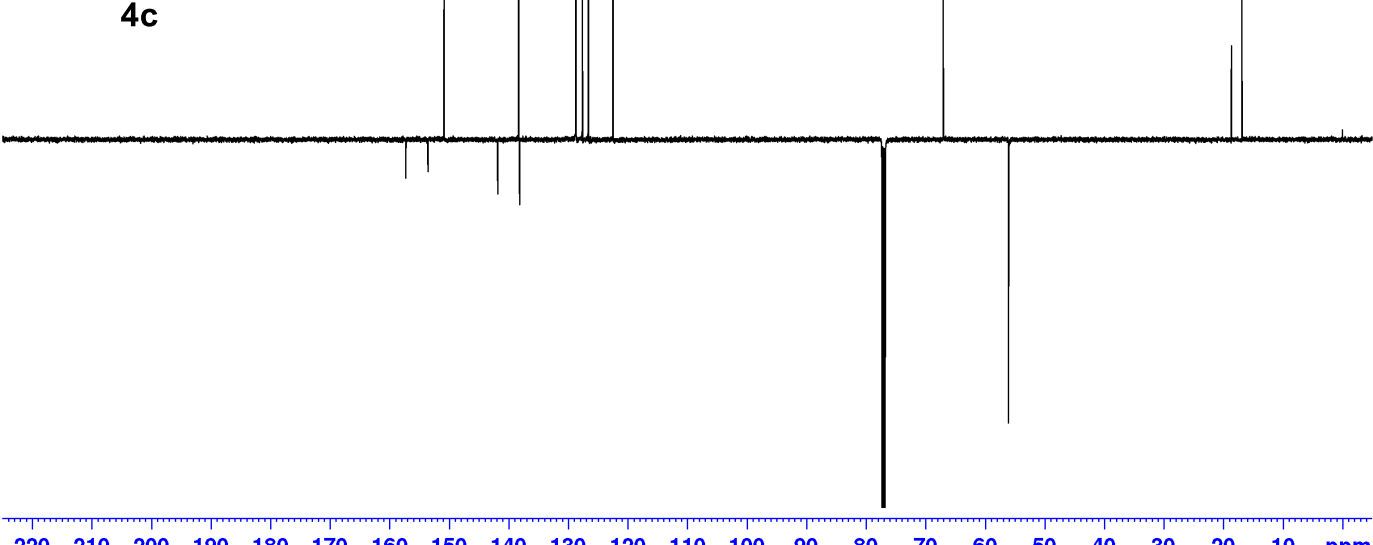




\section{References}

1. Yoshimura, A.; Nemykin V. N.; Zhdankin, V. V. Chem. Eur. J., 2011, 17, 10538.

2. Evans, D. A.; Bilodeau, M. T.; Faul, M. M. J. Am. Chem. Soc. 1994, 116, 2742.

3. Ruppel, J. V.; Jones, J. E.; Huff, C. A.; Kamble, R. M.; Chen Y.; Zhang, X. P. Org. Lett. 2008, 10, 1995.

4. Han, H.; Bae, I.; Yoo, E. J.; Lee, J.; Do, Y.; Chang, S. Org. Lett. 2004, 6, 4109.

5. Gao, G.-Y.; Harden J. D.; Zhang, X. P. Org. Lett. 2005, 7, 3191.

6. Mayer, A. C.; Salit, A.-F.; Bolm, C. Chem. Commun., 2008, 5975.

7. Dolomanov, O. V.; Bourhis, L. J.; Gildea, R. J.; Howard, J. A. K.; Puschmann, H. J. Appl. Cryst. 2009, 42, 339.

8. Sheldrick, G. M. Acta Cryst. 2015, A71, 3.

9. Sheldrick, G. M. Acta Cryst. 2008, A64, 112. 